\title{
Funktionelle Genomanalyse bakterieller Erreger, assoziiert mit der Europäischen Faulbrut von Honigbienen
}

\author{
Dissertation \\ Zur Erlangung des mathematisch-naturwissenschaftlichen Doktorgrades \\ „Doctor rerum naturalium“ \\ der Georg-August-Universität Göttingen \\ im Promotionsprogramm Biologie \\ der Georg-August University School of Science (GAUSS)
}

vorgelegt von

Marvin Djukic

aus Leinefelde

Göttingen, 2015 
Betreuungsausschuss

Prof. Dr. Rolf Daniel, Abteilung für Genomische und Angewandte Mikrobiologie, Institut für Mikrobiologie und Genetik

PD Dr. Michael Hoppert, Abteilung für Allgemeine Mikrobiologie, Institut für Mikrobiologie und Genetik

$\underline{\text { Mitglieder der Prüfungskommission }}$

Referent: $\quad$ Prof. Dr. Rolf Daniel, Abteilung für Genomische und Angewandte Mikrobiologie, Institut für Mikrobiologie und Genetik

Korreferent: PD Dr. Michael Hoppert, Abteilung für Allgemeine Mikrobiologie, Institut für Mikrobiologie und Genetik

Weitere Mitglieder der Prüfungskommission:

Prof. Dr. Gerhard Braus, Abteilung Molekulare Mikrobiologie und Genetik, Institut für Mikrobiologie und Genetik

Prof. Dr. Hermann Behling, Abteilung für Palynologie und Klimadynamik, Albrecht-vonHaller Institut für Pflanzenwissenschaften

Jun.-Prof. Dr. Kai Heimel, Abteilung für Molekulare Mikrobiologie und Genetik, Institut für Mikrobiologie und Genetik

PD Dr. Wilfried Kramer, Abteilung für Molekulare Genetik, Institut für Mikrobiologie und Genetik

Tag der mündlichen Prüfung: $\quad$ 07.10.2015

Beginn: 10:00 Uhr 


\section{Inhaltsverzeichnis}

Inhaltsverzeichnis

Publikationsliste

A EINLEITUNG 1

1 Bedeutung der Honigbiene 1

2 Biologie der Honigbiene 2

3 Völkerverluste und Bienenkrankheiten $\quad 6$

3.1 Amerikanische (AFB) und Europäische Faulbrut (EFB) 8

4 Zielstellung 13

5 Referenzen 14

B PUBLIKATIONEN

1 Die andere Faulbrut $\quad 20$

2 How to Kill the Honey Bee Larva: Genomic Potential and Virulence 22 Mechanisms of Paenibacillus larvae

2.1 Ergänzende Daten zu Kapitel B2

3 Paenilarvins: Iturin Family Lipopeptides from the Honey Bee 53 Pathogen Paenibacillus larvae

3.1 Ergänzende Daten zu Kapitel B3

4 How to kill the honey bee larvae: genomic potential and virulence factors of Melissococcus plutonius

4.1 Ergänzende Daten zu Kapitel B4

5 Genome Sequence of Brevibacillus laterosporus LMG 15441, a Pathogen of Invertebrates

6 Genome Sequence of Paenibacillus alvei DSM 29, a Secondary Invader during European Foulbrood Outbreaks 
7 High quality draft genome of Lactobacillus kunkeei EFB6, isolated from a German European foulbrood outbreak of honeybees

7.1 Ergänzende Daten zu Kapitel B7

8 First Insights into the Genome of Fructobacillus sp. EFB-N1, Isolated from Honey Bee Larva Infected with European Foulbrood

C DISKUSSION

135

1 Unterschiede und Gemeinsamkeiten zwischen AFB und EFB:

Funktionelle und vergleichende Genomanalyse von P. larvae (AFB) und M. plutonius (EFB)

1.1 Nahrungsaufnahme und Nicht-invasive Phase der AFB- und EFBPathogenese

1.1.1 Überdauerungsformen bei P. larvae und M. plutonius

1.1.2 Konkurrenzkampf im Darm der Honigbienenlarve

1.1.3 Energie- und Zuckermetabolismus

1.1.4 Tyramin als frühzeitig sekretierter, potentieller Virulenzfaktor

1.2 Invasive Phase der AFB- und EFB-Pathogenese

1.2.1 Die Degradation der peritrophischen Matrix (PM) der

Honigbienenlarve als Übergang zur invasiven Phase

1.2.2 Durchbruch durch das Darmepithel und die Zersetzung des

Wirtskörpers

1.3 Genomreduktion als Zeichen der Evolution: Der Einfluss von Phagen, mobilen genetischen Elementen und Nonsens-Mutationen auf die Virulenz der Erreger

2 Initiale Genomanalyse von Sekundärerregern der Europäischen Faulbrut

3 Die Rolle von Lactobacillus kunkeei als potentieller EFB-Antagonist und Fructobacillus sp. EFB-N1 als fructophiles Bakterium

4 Ausblick

5 Referenzen 
E APPENDIX 


\section{Publikationsliste}

Djukic, M., Poehlein, A., Thürmer, A., Daniel, R. 2011. Genome Sequence of Brevibacillus laterosporus LMG 15441, a pathogen of invertebrates. J. Bacteriol. 193, 5535-5536.

Djukic, M., Becker, D., Poehlein, A., Voget, S., Daniel, R. 2012. Genome Sequence of Paenibacillus alvei DSM 29, a Secondary Invader during European Foulbrood Outbreaks. J. Bacteriol. 194, 6365.

Djukic, M., Hartken D. 2013. Die andere Faulbrut. Deutsches Bienenjournal 8, 17.

Djukic, M., Brzuszkiewicz, E., Fünfhaus, A., Voss, J., Gollnow, K., Poppinga, L., Liesegang, H., Garcia-Gonzalez, E., Genersch, E., Daniel, R. 2014. How to Kill the Honey Bee Larva: Genomic Potential and Virulence Mechanisms of Paenibacillus larvae. PLoS One 9, e90914.

Sood, S., Steinmetz, H., Beims, H., Mohr, K. I., Stadler, M., Djukic, M., von der Ohe, W., Steinert, M., Daniel, R., Müller, R. 2014. Paenilarvins: Iturin Family Lipopeptides from the Honey Bee Pathogen Paenibacillus larvae. ChemBioChem 15, 1947-1955.

Djukic, M., Poehlein, A., Strauß, J., Tann, F., Leimbach, A., Hoppert, M., Daniel, R. 2015. High quality draft genome of Lactobacillus kunkeei EFB6, isolated from a German European foulbrood outbreak of honeybees. Stand. Genomic Sci. 10, 16.

Djukic, M., Daniel, R., Poehlein, A. 2015. First Insights into the Genome of Fructobacillus sp. EFB-N1, Isolated from Honey Bee Larva Infected with European Foulbrood. Genome Announc. 3, 10-11.

Djukic, M., Leimbach, A., Grossar, D., Charrière, J-D., Gauthier, L., Hartken, D., Poehlein A., Daniel, R. 2015. How to kill honey bee larvae: genomic potential and virulence factors of Melissococcus plutonius. 


\section{A Einleitung}

\section{Bedeutung der Honigbiene}

„Und der HERR sprach: Ich habe das Elend meines Volks in Ägypten gesehen und ihr Geschrei über ihre Bedränger gehört; ich habe ihre Leiden erkannt. Und ich bin herniedergefahren, dass ich sie errette aus der Ägypter Hand und sie herausführe aus diesem Lande in ein gutes und weites Land, in ein Land, darin Milch und Honig fließt, $[\ldots] . “$

Luther Bibel, Exodus/2. Mose, Kapitel 3, Verse 7 und 8.
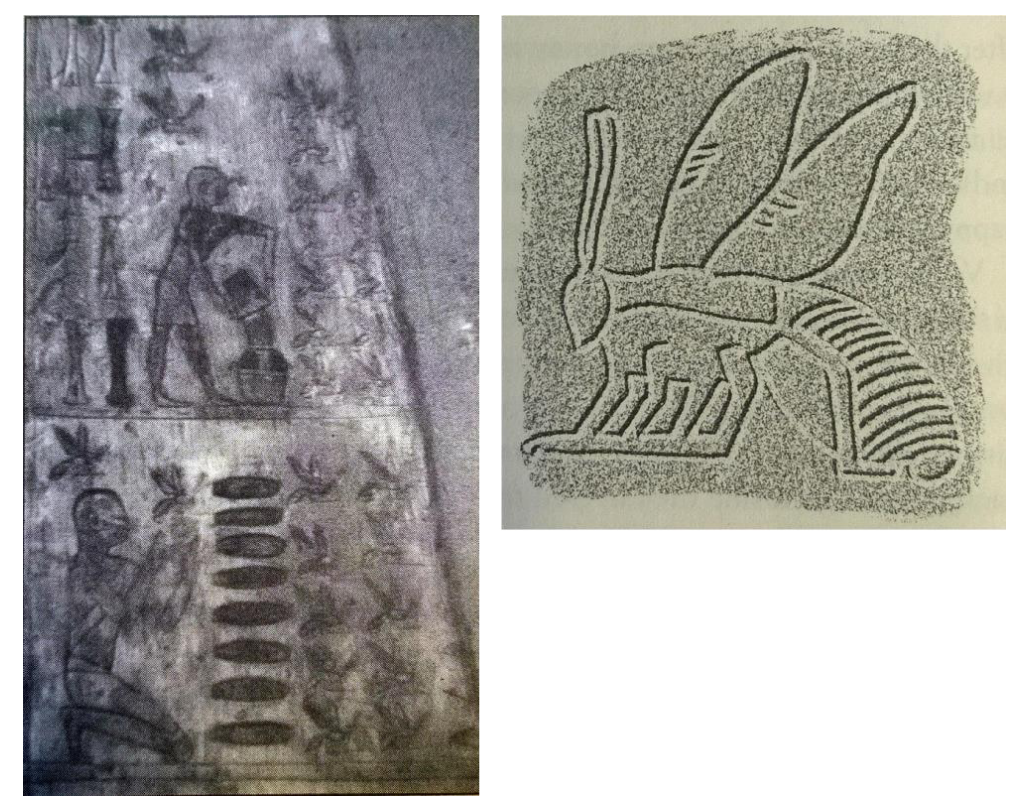

Abbildung 1: Honigbienenverzierungen im Alten Ägypten.

Die linke Abbildung (oberer Abschnitt) zeigt einen ägyptischen Imker, der Honig in einen Behälter überführt. Um ihn herum schwirren Honigbienen (oberer und unterer Abschnitt). Diese Ritzverzierung stammt von einer Steinsäule, am Grab von Pabesa, ca. 650 vor Christus. Die rechte Abbildung zeigt die Hieroglyphe einer Honigbiene, ein Symbol für Mitglieder des Königshauses, am Grab von Pharao Intef, ca. 1550 vor Christus. (Quelle: Buchmann, 2005, Letters from the Hive)

Honigbienen (Apis) gehören zusammen mit den Hummeln zu der Familie der Echten Bienen (Apidae). Die Artenvielfalt ist gering, da weltweit nur neun Spezies der Gattung Apis bekannt sind. In Asien leben acht Arten von Honigbienen, während es in Europa und Afrika nur eine einzige Spezies gibt, Apis mellifera, auch die Westliche oder Europäische Honigbiene genannt (Tautz, 2012). Im Laufe der Zeit haben sich zahlreiche Subspezies dieser Art entwickelt und wurden dann durch den Menschen weltweit verbreitet (Tautz, 2012). Ob im Alten Ägypten (Abbildung 1), antiken Griechenland oder Rom, ob in der Bibel oder dem Koran, dem Hinduismus oder Buddhismus, seit Jahrtausenden wird die 
Honigbiene von dem Menschen als Nutztier gehalten und in schriftlichen und bildlichen Überlieferungen erwähnt.

Zusammen mit ca. 560 weiteren, heimischen Wildbienenarten sorgt die Westliche Honigbiene für Vielfalt in der Natur und ist in Deutschlands Ökosystemen und Landschaften unverzichtbar (Bundesministerium für Ernährung und Landwirtschaft, 2014). Die Honigbiene ist derzeit nach Rind und Schwein das drittwichtigste Nutztier für die Ernährung des Menschen in Europa (Tautz, 2012). Sie spielt eine entscheidende Rolle bei der Bestäubung von Pflanzen und der Nahrungsmittelproduktion. Wenngleich viele zur Nahrungsmittelproduktion verwendete Pflanzen, wie z.B. Getreide, Reis und Tomaten, unabhängig von einer durch Tiere vermittelten Bestäubungstätigkeit sind, so darf man den Beitrag zu einer ausgeglichenen, gesunden Ernährung nicht vergessen. Etwa $35 \%$ aller Nutzpflanzen sind auf die Bestäubungstätigkeit angewiesen (Klein et al., 2007). Dazu gehören u.a. Äpfel, Birnen, Süß- und Sauerkirschen, Aprikosen, Pflaumen, Pfirsiche, Nektarinen, Himbeeren, Brombeeren, Melonen und Kiwis, sowie Gurken, Zucchini, Mandeln, Fenchel und Koriander. Achtzig Prozent aller Blütenpflanzen werden von Insekten bestäubt und von diesen wiederum etwa $85 \%$ von Honigbienen (Tautz, 2012). Eine Studie von Gallai und Kollegen bezifferte den Wert der durch Insekten vermittelten Bestäubung bei Nutzpflanzen im Jahr 2005 auf 153 Milliarden Euro, welche 9,5 \% der Weltnahrungsmittelproduktion widerspiegeln (Gallai et al., 2009). Nahezu $90 \%$ der kommerziellen Bestäubungstätigkeit gehen auf von Menschen gehaltene Honigbienen zurück (Genersch, 2010b; Steffan-Dewenter et al., 2005). Dadurch sind Honigbienen die wichtigsten, kommerziell genutzten Pflanzenbestäuber weltweit (Steffan-Dewenter et al., 2005). Auch sind Honigbienen für die Bestäubung von Pflanzen zuständig, deren Früchte als Nahrungsmittel für weitere Tiere von Bedeutung sind, beispielsweise Vogelbeeren bei Vögeln oder verrottende Früchte bei Fruchtfliegen. Sie tragen außerdem zur Verbreitung des Produzenten bei.

\section{Biologie der Honigbiene}

Die Honigbiene ist eine der am besten erforschten Insektenarten der Welt. Im Sommer legt eine Honigbienenkönigin täglich zwischen 1.000 und 2.000 Eier. Dabei entstehen aus unbefruchteten Eiern die männlichen Drohnen und aus Befruchteten Königinnen oder Arbeiterinnen. Die Entwicklung erfolgt in vier aufeinanderfolgenden Stadien: das Ei, die Larve, die Puppe und die adulte Honigbiene. Die vollständige Entwicklung bis hin zur 
adulten Honigbiene dauert bei Arbeiterinnen ungefähr 21 Tage (Abbildung 2), bei Königinnen 16 Tage und bei den Drohnen 24 Tage.

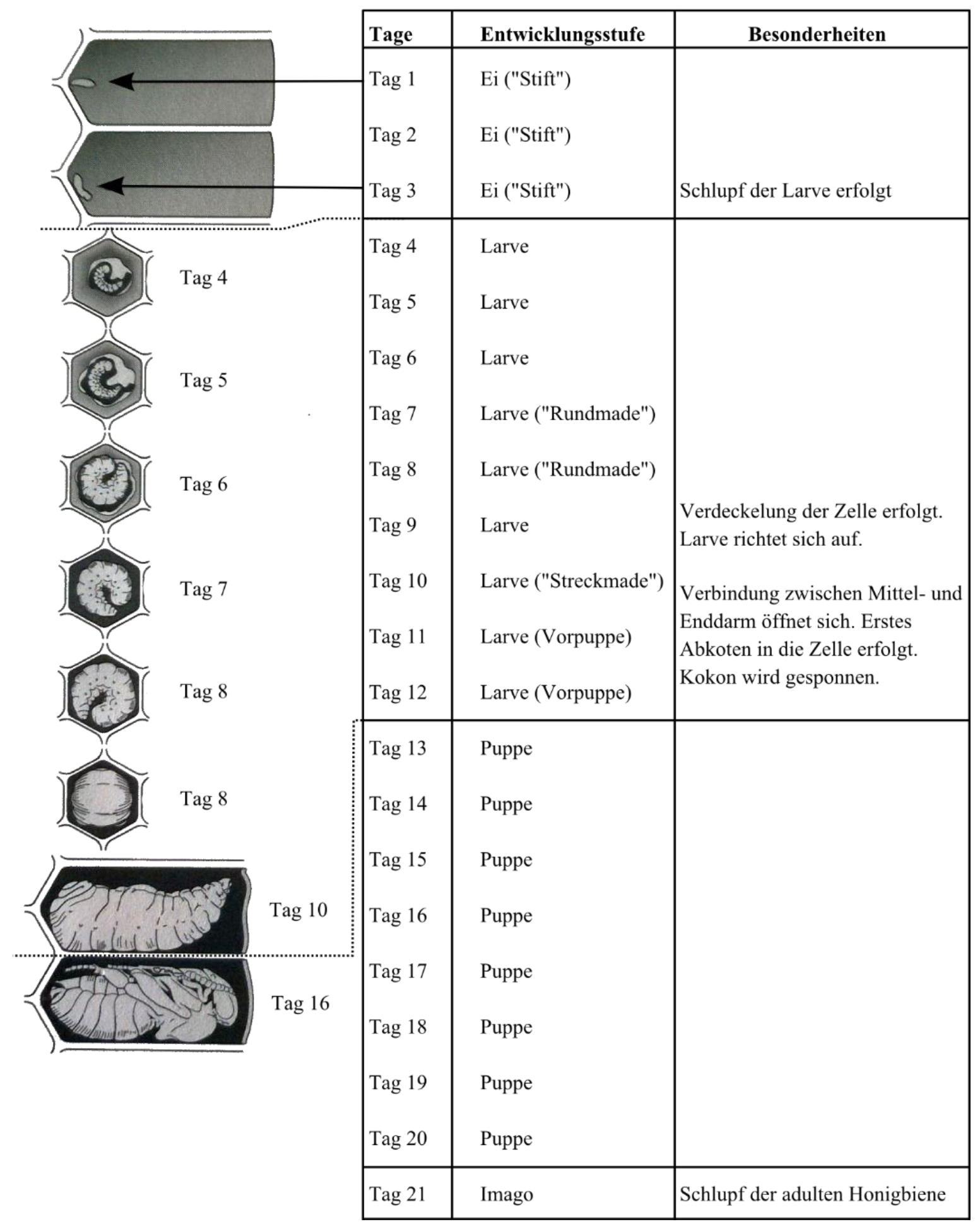

Abbildung 2: Die Entwicklung der Arbeiterinnenbienenbrut (editierte Abbildung aus Ritter 2012). Oft verwendete, umgangssprachliche Bezeichnungen der jeweiligen Stadien sind mit Anführungszeichen gekennzeichnet. Während die Stadien bei Drohne, Königin und Arbeiterin dieselben sind (Ei, Larve, Puppe und adulte Honigbiene), unterscheiden sich die Entwicklungszeiten. Diese liegt bei der Arbeiterin (Apis mellifera) bei 21 Tagen. 
Junglarven (vierter bis sechster Tag in der Honigbienenentwicklung), die frisch aus dem Ei geschlüpft sind, werden von den sogenannten Ammenbienen mit Gelee Royale (,Weiselfuttersaft“) ernährt. Gelee Royale ist ein Sekretgemisch, dessen Bestandteile in speziellen Drüsen (Hypopharynxdrüse und Mandibeldrüse) im Kopf der Ammenbiene durch partiellen Verdau von Pollen und Nektar erzeugt werden (Tautz, 2012). Dieses Gemisch besteht hauptsächlich aus Wasser (ca. 50-60 \%), Zucker (15\%), Proteinen (15 $\%)$, Fetten (3-6 \%), Mineralsalzen (1,5\%), Vitaminen und Aminosäuren (Nagai \& Inoue, 2004; Viuda-Martos et al., 2008), aber auch antibakterielle und antimykotische Peptide, und Immunsystem-modulierende Stoffe sind vorhanden (Bloodworth et al., 1995; Ferlat et al., 1994; Genç \& Aslan, 1999; Sugiyama et al., 2012). Während zukünftige Bienenköniginnen über den dritten Tag der larvalen Entwicklung hinaus mit Gelee Royale gefüttert werden, bekommen die zukünftigen Arbeiterinnen hingegen Arbeiterfuttersaft, der zu einen höheren Anteil Bienenbrot (eingelagerter Pollen) und Honig enthält. Dieser Unterschied ist essentiell, da die Gabe von Arbeiterfuttersaft bzw. Gelee Royale, die Menge des Futters aber auch der Hexose-Anteil im Arbeiterfuttersaft entweder zu einer Entwicklung der Larve zur Königin oder zur Arbeiterin führen (Tautz, 2012).

Am neunten Tag wird eine Arbeiterinnenzelle gedeckelt. Die Larve richtet sich auf und wird übergangsweise Streckmade genannt. In dieser Zeit öffnet sich die bis dahin verschlossene Verbindung zwischen dem Mitteldarm und dem Enddarm (Abbildung 3). Die Larve kotet das erste Mal ab. Außerdem spinnt die Larve einen Kokon. Diese Schritte sind für die Pathogenese einiger Krankheiten von besonderer Bedeutung (siehe Abschnitt A 3.1).

Nach der Streckmade erfolgt das Stadium der Vorpuppe und Puppe. Die Puppe ernährt sich ausschließlich von den Energiereserven, die sie als Larve angelegt hat. Ungefähr zwölf bis dreizehn Tage nach der Verdeckelung der Zelle schlüpft in einem letzten Schritt die adulte Honigbiene (Ritter, 2012). 

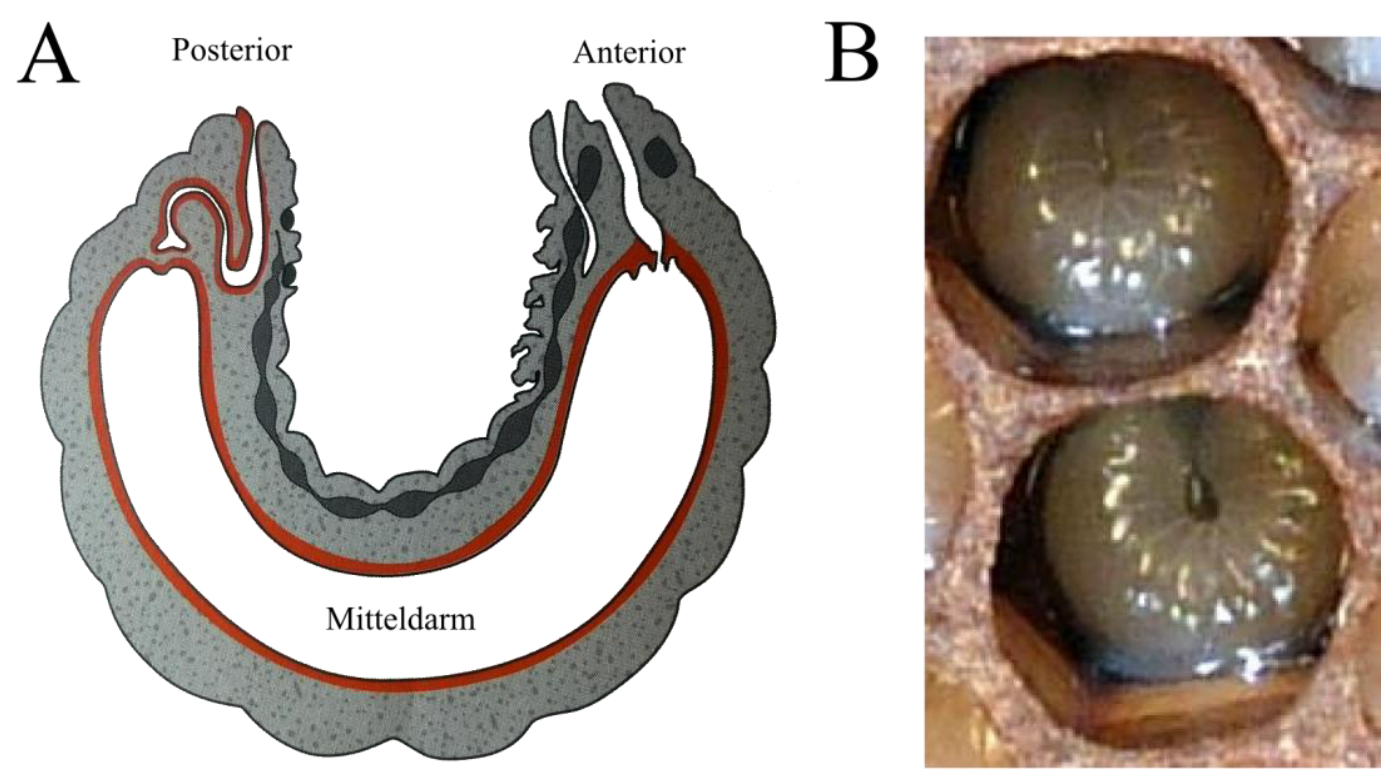

Abbildung 3: Honigbienenlarve in Nahaufnahme.

Abbildung A (editierte Abbildung aus Ritter 2012) zeigt den Längsschnitt durch eine Honigbienenlarve (Arbeiterin, ca. achter Tag in der Entwicklung). Der Enddarm ist mit dem Mitteldarm noch nicht verbunden. Der Mitteldarm ist mit einer chitin- und glycoproteinhaltigen peritrophischen Matrix (PM) ausgekleidet. Abbildung B zeigt das ein Foto der Larve im selben Stadium. (Quelle der Fotoaufnahme: Marvin Djukic, 2012)

Wie bereits erwähnt, besteht die in den ersten Tagen der Larvenentwicklung aufgenommene Nahrung aus Gelee Royale und später aus einem Gemisch aus Gelee Royale, Bienenbrot und einer erbrochenen Zuckerlösung aus dem Ammenbienenhonigmagen (Corby-Harris et al., 2014b; Tautz, 2012). Die bakterielle Diversität im Darm der Honigbienenlarve ergibt sich somit durch die über die Ammenbienen vermittelte Nahrung, die von Bakterien im Honigmagen der Ammenbiene, aber auch von Bakterien am Pollen und im Nektar (und damit verbunden, dem gelagertem Futterkranzhonig) abhängig ist (Rokop et al., 2015). Verschiedene Lactobacillus Stämme, Gilliamella apicola, Snodgrasella alvei und Frischella perrara werden mit der charakteristischen, bakteriellen Darmgemeinschaft adulter Honigbienen in Verbindung gebracht (Engel et al., 2013; Martinson et al., 2012). Eine aktuelle Studie von Hroncova und Kollegen zeigte, dass sich die Zusammensetzung der bakteriellen Gemeinschaft im Darm der Larve im Laufe der Larvenentwicklung verändert (Hroncova et al., 2015). So wurden Lactobacillus spp. und G. apicola (Martinson et al., 2012) in ungefähr sechs Tage alten Larven in höherer Abundanz ermittelt als in jüngeren Larven. S. alvei, welches zu der Familie der Neisseriaceae gehört, und F. perrara, der Familie der Orbaceae zugehörig, konnten auch in der Larve gefunden werden. S. alvei hat in den ersten Tagen der larvalen 
Entwicklung eine höhere Abundanz im Darm der Honigbienenlarve als am sechsten Tag. F. perrara zeigte bei ein-Tage-alten Larven und bei sechs Tage alten Larven eine höhere relative Abundanz als G. apicola und S. alvei (Hroncova et al., 2015).

Weiterhin wurde Parasaccharibacter apium, ein Gram-negatives, nicht-motiles, stäbchenförmiges Bakterium, als Symbiont der Honigbiene charakterisiert. Zusammen mit L. kunkeei wurde die Präsenz dieses Bakteriums in dem Honigmagen und der Hypopharynxdrüse von Ammenbienen, aber auch im Gelee Royale nachgewiesen (CorbyHarris et al., 2014a). Zugleich wurde gezeigt, dass Larven, denen P. apium mit artifizieller Nahrung zugeführt wurde, eine höhere Überlebensrate im Vergleich zu Larven aufwiesen, die ohne dieses Bakterium überleben mussten (Corby-Harris et al., 2014b). L. kunkeei wurde außerdem auch in Pollen und Bienenbrot detektiert (Olofsson \& Vásquez, 2008; Vásquez \& Olofsson, 2009; Vásquez et al., 2012), wenngleich eine Detektion wie bei verschiedenen Fructobacillus spp. von Jahreszeit und Blumentyp abhängt (Anderson et al., 2013). Als natürlicher Inhibitor von Paenibacillus larvae und Melissococcus plutonius wird L. kunkeei eine besondere Rolle zu Teil (Forsgren et al., 2009; Vásquez et al., 2012). Auf $P$. larvae und M. plutonius werden im folgenden Abschnitt näher eingegangen.

\section{Völkerverluste und Bienenkrankheiten}

Die weltweite Anzahl an Honigbienenvölkern hat seit den frühen Sechzigern des letzten Jahrhunderts um $45 \%$ zugelegt. Gleichzeitig ist die Anzahl an bestäubungsabhängigen Nutzpflanzen allerdings um $300 \%$ gestiegen (Aizen \& Harder, 2009). Seit den achtziger Jahren des letzten Jahrhunderts ist die Anzahl der von Menschen gehaltenen Honigbienenvölkern in Europa um mehr als $25 \%$ und in den USA um ungefähr $60 \%$ gesunken (Potts et al., 2010). Ein Großteil des Rückgangs ist auf politische und sozioökonomische Hintergründe zurückzuführen (Smith et al., 2014). Unter anderem führte der Zusammenbruch der Sowjetunion $\mathrm{zu}$ geringeren staatlichen Subventionen für Bienenhaltung in ehemaligen Mitgliedsländern, was einen Rückgang von $50 \%$ der Bienenstöcke in den betroffenen Ländern zur Folge hatte. Westeuropa dagegen hatte mit höheren Produktionskosten, einem Konkurrenzkampf mit billigen Importhonig und kostengünstigen, auf Zucker-basierten Produkten zu kämpfen (Smith et al., 2014). Das führte zu einem Rückgang des Imkereigewerbes um $30 \%$ und einen Rückgang von Honigbienenkolonien um $25 \%$ seit 1985 (Aizen \& Harder, 2009; Potts et al., 2010). In Deutschland ist erst seit zwei Jahren eine leichte Erholung zu erkennen, wobei die 
steigende Anzahl an Bienenvölkern auch an eine steigende Anzahl an neuen Imkern gekoppelt ist (Abbildung 4).

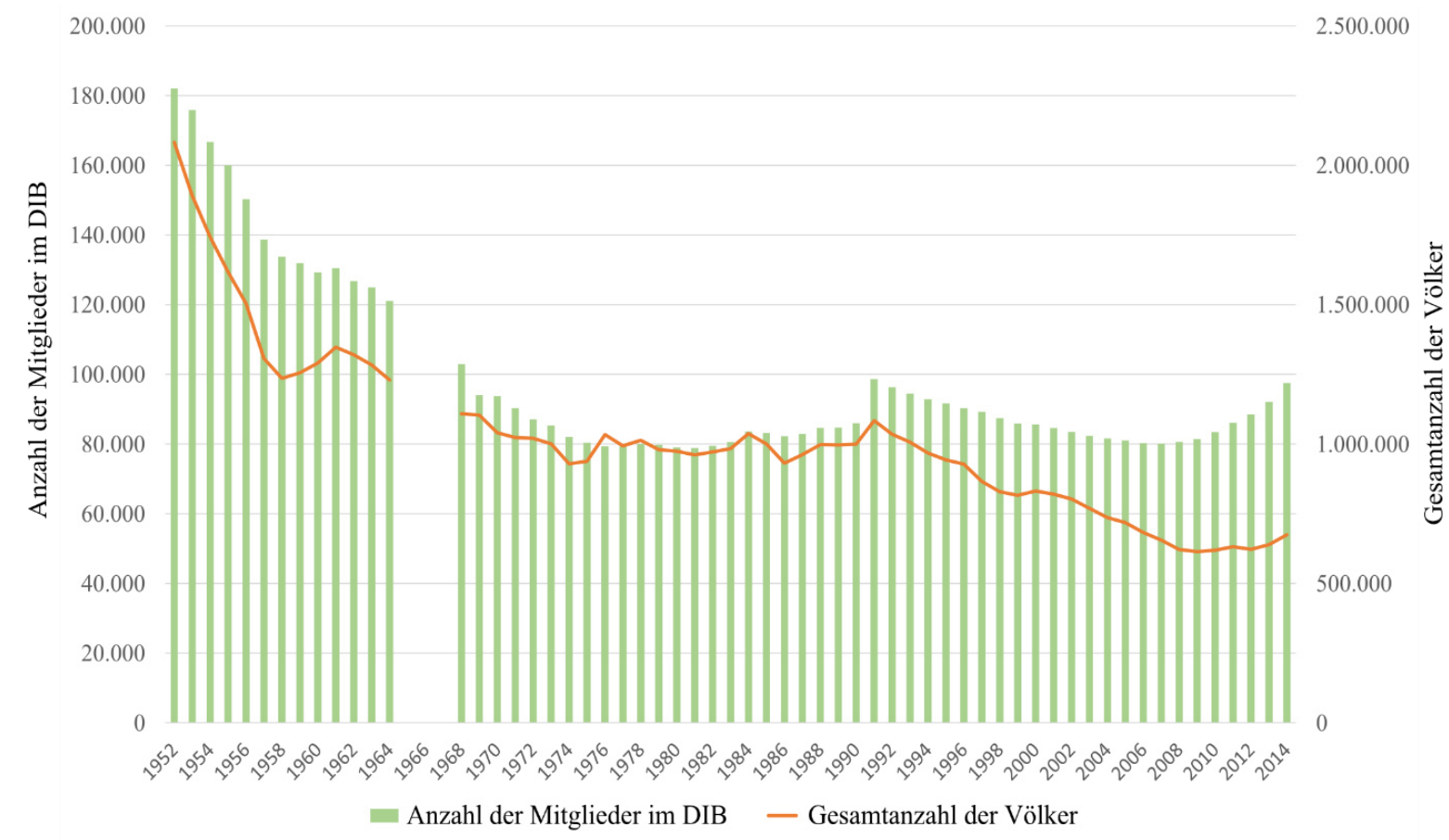

Abbildung 4: Entwicklungsverlauf der Mitgliederanzahl im Deutschen Imkerbund (DIB) und der Anzahl an Bienenvölkern seit 1952 in Deutschland. (Quelle: Deutscher Imkerbund)

Der großflächige Anbau von Monokulturen, die nur einmal im Jahr blühen und der Einsatz von Pestiziden verschlechtern die Situation der Honigbienen in den Industrieländern weiterhin (Barbosa et al., 2015; Blacquière et al., 2012; Cabrera-Marín et al., 2015). Dazu kommt die ansteigende Luftverschmutzung, durch die Honigbienen stark beeinträchtigt werden. Ein weiterer Faktor ist die gängige Imkerpraxis, bei der die natürliche Schwarmbildung unterdrückt wird, aber auch das „,building block principle“, bei dem verschiedene Bienenvölker in eine Bienenbeute zusammengeführt werden. Das führt zu einem massiven Attackieren der Bienen untereinander, bevor diese einen neuen Staat gründen. Des Weiteren führen häufige Standwechsel (Wanderungen) zu Stressempfinden und zu erhöhter Infektionsgefahr. Zusätzlich scheint die Anzahl der durch Krankheiten entstandenen Völkerverluste stetig anzusteigen (Genersch, 2010b). Die parasitisch lebende Milbe „Varroa destructor“ wurde in den Siebzigern und Achtzigern des letzten Jahrhunderts in Europa und Amerika eingeführt und konnte sich massiv verbreiten. Sie wird als einer der Hauptgründe für aktuelle Bienenverluste gesehen und kann Viren wie das „Akute Bienen-Paralyse-Virus“, das „Israeli Akute Bienen-Paralyse-Virus“, das „Flügeldeformationsvirus“ und das „Kashmir Bienenvirus“ übertragen, die ihrerseits auch zu Völkerverlusten führen (Genersch, 2010b; Genersch et al., 2010; Ritter, 2012; Smith et 
al., 2014). Auch die durch das Mikrosporidium Nosema ceranae verursachte Nosemose und die durch die bakteriellen Erreger $P$. larvae und $M$. plutonius verursachte Amerikanische Faulbrut von Honigbienen (AFB) bzw. Europäische Faulbrut von Honigbienen (EFB) sorgen für zahlreiche Völkerverluste bei Apis mellifera.

\subsection{Amerikanische (AFB) und Europäische Faulbrut (EFB)}

Sowohl die AFB als auch die EFB stellen intestinale Infektionen der Honigbienenlarve (Apis mellifera) dar. Während die AFB von dem Gram-positiven, sporenbildenden Bakterium P. larvae hervorgerufen wird (Genersch et al., 2006; Heyndrickx et al., 1996; White, 1906), ist der Auslöser einer EFB Infektion das Gram-positive, kapselbildende Bakterium M. plutonius (Bailey, 1983; Ritter, 2012; White, 1912).

Bereits vor über 2000 Jahren wurden im antiken Griechenland und Rom Bienenseuchen beschrieben, mitunter auch Symptome, die der AFB zuzuordnen sind (Genersch, 2008). Die AFB gilt als hochansteckend und ist in vielen Ländern eine anzeigepflichtige Seuche. Sie wird umgangssprachlich „Bösartige Faulbrut“ genannt. Nur die Endosporen des Erregers sind infektiös (Genersch, 2008) und werden über kontaminiertes Futter aufgenommen. Bereits ungefähr zehn Sporen reichen aus, um eine Infektion mit anschließendem Tod der Larve hervorzurufen (Woodrow, 1942). Puppen und adulte Honigbienen sind von dieser Seuche nicht betroffen. Die Larven sind ca. zwölf bis 36 Stunden nach dem Eischlupf am anfälligsten für AFB (Genersch, 2010a). Nach der Infektion erfolgt die typische Pathogenese. Zuerst keimen im Mitteldarm der Larve die Sporen aus. In diesem frühen Stadium, auch das nicht-invasive Stadium genannt, leben die vegetativen Zellen vom zuckerreichen Futter im Darm der Larve, ohne diese dabei aktiv zu schädigen (Genersch, 2010a; Poppinga \& Genersch, 2015). In der darauffolgenden, invasiven Phase wird in einem ersten Schritt die peritrophische Matrix (PM) angegriffen und durchbrochen (Yue et al., 2008). Diese im Mittdeldarm vorkommende, chitin- und glycoproteinhaltige Barriere schützt das restliche Gewebe der Larve vor Verdauungsprozessen, aber auch vor Pathogenen und Toxinen (Hegedus et al., 2009; Terra, 2001). Der Darm der Larve ist zu diesem Zeitpunkt bereits mit vegetativen Zellen und Sporen des Erregers ausgefüllt. Als nächstes wird die Epithelschicht angegriffen (Genersch, 2010a). Das Haemocoel wird nachfolgend über eine parazellulare Route erreicht (Genersch, 2010a). Der Aufschluss der Epithelschicht und das Eindringen in das Haemocoel mit anschließender Degradierung des Gewebes gehen mit dem Tod der Larve einher. Von der ursprünglichen weißen Larve bleibt nach wenigen Tagen nur noch eine 
bräunliche, fadenziehende Masse übrig. Diese Masse trocknet ein und enthält Millionen von Sporen des Keims (Bailey \& Ball, 1991; Lindström et al., 2008). Die Sporen bleiben dabei für über 35 Jahre infektiös (Hasemann, 1961). Zudem gelingt es P. larvae, sich gegen weitere Sekundärerreger bzw. Saprophyten durchzusetzen, so dass die Gewinnung von Reinkulturen des Erregers aus an AFB-verstorbenen Larvenresten möglich ist (Poppinga \& Genersch, 2015).

Im Laufe der letzten Jahre hat sich die Klassifizierung des Erregers in verschiedene „ERIC“-Genotypen weitgehend verbreitet. Diese Einteilung ist in epidemiologischen Studien durch die Verwendung von „Enterobacterial Repetitive Intergenic Consensus“Oligonukleotiden bei der sogenannten „repetitive element“-PCR (Hulton et al., 1991; Sharples \& Lloyd, 1990) entstanden. Insgesamt vier Genotypen konnten so ermittelt werden (ERIC I - IV), wobei nur ERIC I und II regelmäßig aus AFB-infizierten Bienenvölkern im Freiland isoliert werden und somit der Fokus der Forschung auf diesen beiden Genotypen liegt (Poppinga \& Genersch, 2015). Versuche von Genersch und Kollegen haben gezeigt, dass sich die verschiedenen Genotypen von $P$. larvae in ihrer Virulenz stark unterscheiden, die Mortalität bei einer Infektion allerdings gleich bleibt (Genersch et al., 2005, 2006). Stämme des ERIC II- Genotyps zeigten dabei grundsätzlich die höchste Virulenz und waren in der Lage, alle infizierten Larven nach ca. sieben Tagen abzutöten (Ashiralieva \& Genersch, 2006; Genersch, 2010a; Genersch et al., 2005). Gleichzeitig bedeutet das, dass P. larvae ERIC II-infizierte Larven bereits in einem ungedeckelten Zustand absterben, da die Deckelung erst nach neun Tagen erfolgt. Im Gegensatz dazu benötigen Stämme des Genotyps ERIC I ungefähr zwölf Tage, um alle infizierte Brut zu töten. Auf Larvenebene wurde dem ERIC I-Genotyp somit eine geringere Virulenz zugeschrieben (Ashiralieva \& Genersch, 2006; Genersch et al., 2005). Diese korreliert allerdings nicht mit der Virulenz auf Volkebene. Das verzögerte Absterben der Larve bei einer Infektion mit dem ERIC I Genotyp führt zu einer wesentlich schlechteren Erkennungs- und Entfernungsrate durch die Arbeiterinnen im Bienenvolk, da die Zellen dieser Larven bereits gedeckelt wurden. Rauch und Kollegen konnten in einer Studie zeigen, dass neun von zehn ERIC II-infizierte Larven von Arbeiterinnen erkannt und entfernt werden, dagegen aber nur sechs von zehn mit ERIC I-infizierten Larven (Rauch et al., 2009). Je mehr und je früher infizierte Larven erkannt und entfernt werden, je langsamer erfolgt die weitere Pathogenese, da die Übertragung des Erregers auf weitere Larven verlangsamt wird. Somit besitzen ERIC I Genotypen zwar eine niedrige Virulenz 
auf Larvenebene, aber eine hohe Virulenz auf Volkebene, da die Übertragung des Erregers innerhalb des Volkes schneller vonstattengeht (Rauch et al., 2009).

Im Gegensatz zu der AFB wird der EFB eine geringere Bedeutung, Virulenz und Mortalität zugeschrieben. Sie wird daher umgangssprachlich als „Gutartige Faulbrut“ bezeichnet. Wie bei der AFB, gelangt der Erreger über kontaminiertes Futter in die Larve, wobei die Anfälligkeit für eine Infektion mit steigendem Larvenalter abnimmt (Genersch, 2010b). Laut Bailey sind weniger als 100 vegetative Zellen von M. plutonius notwendig, um eine Infektion auszulösen (Bailey, 1960). Im Allgemeinen sterben infizierte Larven innerhalb von vier bis fünf Tagen (Bailey \& Ball, 1991), also noch vor der Verdeckelung der Zelle, und werden von Arbeiterinnen entfernt. Dadurch wird eine weitere Verbreitung des Erregers im Bienenvolk unterbunden. Ähnlich zur AFB ist es aber auch möglich, dass die Larve erst in der gedeckelten Zelle stirbt. Im Unterschied zur AFB muss eine Infektion aber nicht immer letal verlaufen (Forsgren, 2010). Es ist bekannt, dass Larven eine Infektion überstehen können (Forsgren, 2010). So können auch infizierte Larven abkoten, allerdings spinnen diese häufig einen unvollständigen Kokon, der der Puppe nicht ausreichend Schutz vor Sekundärinfektionen bietet (Ritter, 2012). Tritt keine weitere Infektion auf, sind die aus den infizierten Larven hervorgehenden, adulten Bienen oftmals kleinwüchsig. Die Übertragung von M. plutonius innerhalb des Bienenstocks erfolgt über den Kot der Larven, die kurz vor der Verpuppung stehen (Forsgren, 2010). Der Erreger kann im Kot über Jahre hinweg keimfähig bleiben (Ritter, 2012). Arbeiterinnen, die sich mit der Brutpflege beschäftigen, können zudem eine hohe Keimbelastung aufweisen und den Erreger verschleppen (Belloy et al., 2007; McKee et al., 2003). Im Gegensatz zur AFB ist bei der EFB die Pathogenese noch nicht geklärt. Bakterien wie Enterococcus faecalis, Paenibacillus alvei, Brevibacillus laterosporus und Achromobacter eurydice werden regelmäßig als Sekundärerreger bei der EFB beschrieben und können aus EFB-infizierten oder verstorbenen Larven isoliert werden (Bailey, 1963). Jedes dieser Bakterien wurde bereits als Primärerreger der EFB vermutet, jedoch konnte gezeigt werden, dass nur $M$. plutonius die klassischen EFB-Symptome auslöst (Bailey and Locher, 1968; Bailey, 1963; Forsgren, 2010) und zum Tod der Larve führt. Zu den Symptomen gehört u.a. die Gelbfärbung infizierter Larven innerhalb weniger Tage, die später in eine Braunfärbung umschlägt (Abbildung 5). Bei genauer Betrachtung wird zudem das Tracheensystem sichtbar. Infizierte Larven nehmen oftmals eine ungewöhnliche, seitliche Position innerhalb ihrer Zelle ein. Nach dem Tod der Larve trocknet diese, im Gegensatz zur AFB, 
schnell ein und bildet einen lockeren Schorf, der leicht aus der Zelle entfernt werden kann (Ritter, 2012).

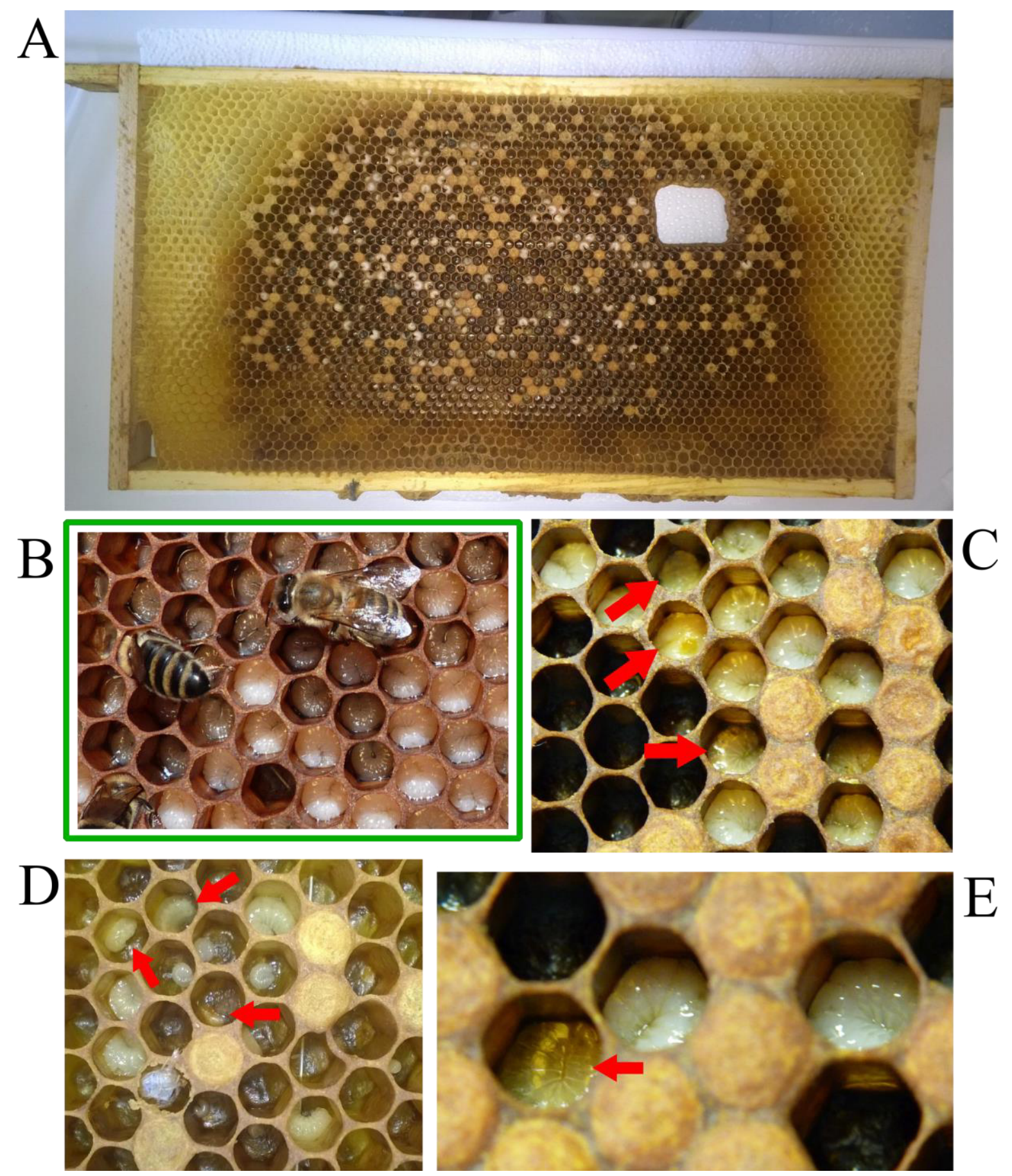

Abbildung 5: Fotoaufnahmen von gesunden (B) und EFB-infizierten (A, C, D, E) Honigbienenvölkern (Apis mellifera).

Abbildung A zeigt das typische, lückenhafte Brutbild, welches sich einerseits durch das Entfernen von EFB-infizierten Larven durch die Arbeiterinnen ergibt, andererseits auch durch die Überreste von verstorbenen Larven, die Zellen besetzen. Abbildung B (grün umrandet) zeigt ein gesundes Volk. Die Larven erscheinen weiß und liegen flach auf dem Grund der Zellen. Larven benachbarter Zellen weisen ungefähr das gleiche Alter auf. EFB-infizierte Larven besitzen zum einen eine Gelbfärbung und zum anderen ist das Tracheensystem sichtbar (potentiell infizierte Larven sind durch rote Pfeile in Abbildung C, D, und E markiert). Außerdem liegen EFB-infizierte Larven 
oftmals verschoben an den Rand ihrer Zellen (Abbildung C und D). Im späteren Verlauf trocknen verstorbene Larven ein (Abbildung D, unterster roter Pfeil) und nehmen eine bräunliche Färbung an. (Quelle der Fotoaufnahmen: Marvin Djukic, 2012-2014)

Im Jahr 2012 veröffentlichen Arai und Kollegen eine Studie über eine Klassifizierung von typischen und atypischen M. plutonius Stämmen (Arai et al., 2012). Typische Stämme unterscheiden sich von Atypischen insofern, dass sie weniger virulent sind und ihre Virulenz nach mehrmaliger Anzucht in Kulturmedien verloren geht. Außerdem wachsen sie nur unter mikroaerophilen bis anaeroben Bedingungen und unter Zugabe von Kaliumphosphat an (Arai et al., 2012; Takamatsu et al., 2013). Ein Jahr später folgte die erste Studie über eine „Multi-locus Sequenztypisierung“ (MLST) von M. plutonius Stämmen (Haynes et al., 2013), die zu weiterführenden, epidemiologischen Untersuchungen beitragen soll. Bis Ende 2014 wurden so 352 Isolaten aus 17 verschiedenen Ländern ein Sequenztyp (ST) zugeordnet und in drei klonale Komplexe (CC) eingeordnet (Takamatsu et al., 2014) (Abbildung 6).
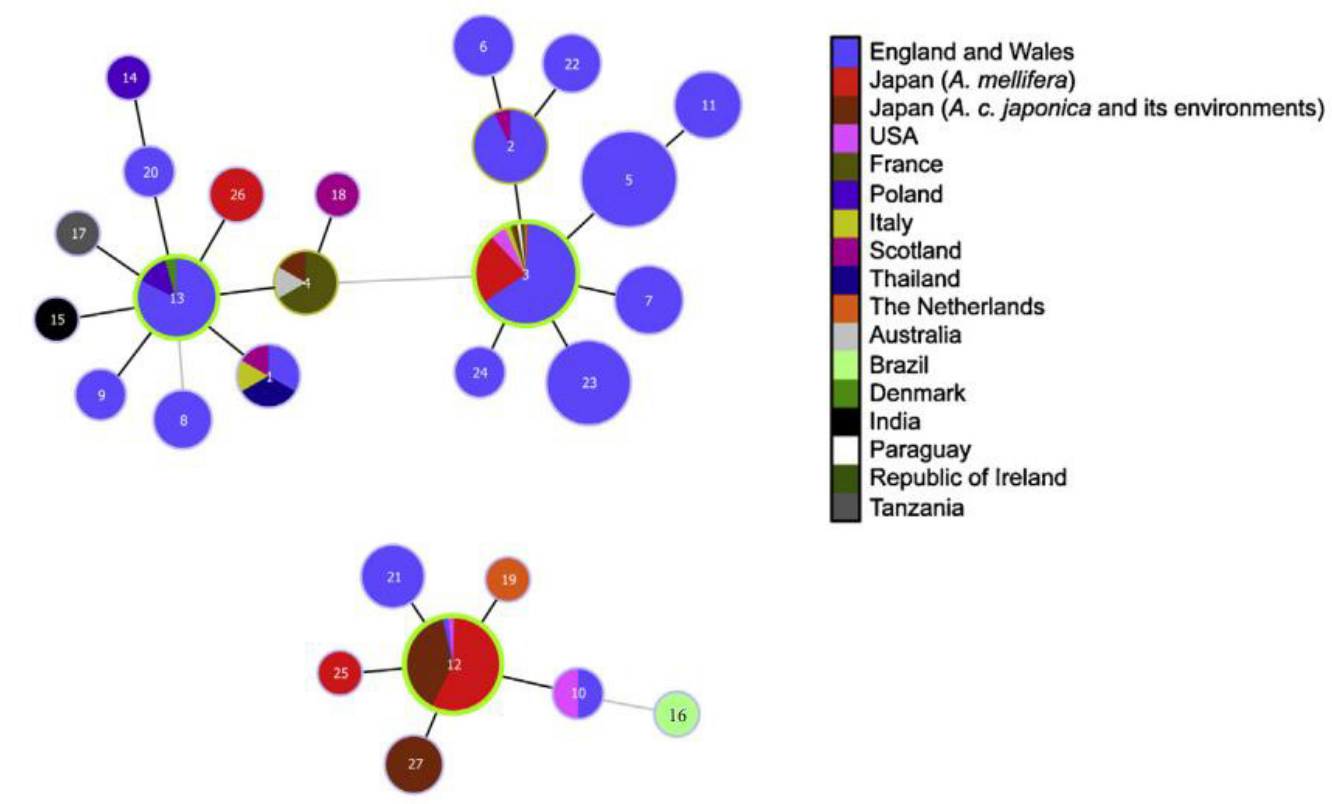

\begin{abstract}
Abbildung 6: Minimaler Spannbaum von ermittelten M. plutonius STs (Francisco et al., 2012; Takamatsu et al., 2014).

Jeder Kreis repräsentiert einen eigenen ST. Die Linien verbinden jeweils die nächsten Verwandten. Schwarze Linien zeigen eine Mutation in einem einzelnen Allel und graue Linien zeigen Unterschiede in zwei Allelen. Grün umrandete Kreis zeigen sogenannte Gründergenotypen. Der Durchmesser der Kreise repräsentiert die relative Abundanz des jeweiligen ST. Die Farben innerhalb eines Kreises markieren die Herkunft der M. plutonius-Isolate (siehe die Legende auf der rechten Seite). Die Abbildung entstammt aus der Veröffentlichung von Takamatsu et al. (2014).
\end{abstract}




\section{Zielstellung}

Das Ziel dieser Arbeit war die Ermittlung von Virulenzfaktoren der Primärerreger der AFB und EFB, P. larvae bzw. M. plutonius. Die ermittelten Virulenzfaktoren sollten der Pathogenese der jeweiligen Krankheit zugeordnet werden, um ein Infektions- und Verlaufsmodell $\mathrm{zu}$ erstellen. Ein weiterer Fokus lag auf den Virulenzunterschieden zwischen den Genotypen ERIC I und ERIC II ( $P$. larvae) und den atypischen und typischen Stämmen von M. plutonius. Die Basis für die Ermittlung und Zuordnung dieser Virulenzfaktoren war die vergleichende und funktionelle Genomanalyse von vierzehn $M$. plutonius und zwei $P$. larvae Stämmen des ERIC I und ERIC II-Genotyps. Im Rahmen der Arbeit erfolgten hierfür auch Isolierungen von $M$. plutonius Stämmen aus Larven, die aus einem Schweizer EFB-Ausbruch stammten. Ferner wurden die Transkriptionslevel von potentiellen Virulenzfaktoren mit Hilfe von RT-PCR untersucht.

Des Weiteren wurden initiale Genomanalysen der EFB-Sekundärerreger Br. laterosporus und $P$. alvei durchgeführt. Auch hier stand die Identifizierung von potentiellen Virulenzfaktoren im Vordergrund. Darüber hinaus wurden die Stämme L. kunkeei EFB6 und Fructobacillus sp. EFB-N1 aus Deutschen und Schweizer EFB-infizierten Honigbienenlarven isoliert und als Erste ihrer Art einer Genomanalyse unterzogen. 


\section{Referenzen}

Aizen, M. A., Harder, L. D. (2009). The global stock of domesticated honey bees is growing slower than agricultural demand for pollination. Curr. Biol. 19, 915-8.

Anderson, K. E., Sheehan, T. H., Mott, B. M., Maes, P., Snyder, L., et al. (2013). Microbial ecology of the hive and pollination landscape: bacterial associates from floral nectar, the alimentary tract and stored food of honey bees (Apis mellifera). PLoS One 8, e83125.

Arai, R., Tominaga, K., Wu, M., Okura, M., Ito, K., et al. (2012). Diversity of Melissococcus plutonius from Honeybee Larvae in Japan and Experimental Reproduction of European Foulbrood with Cultured Atypical Isolates. PLoS One 7, e33708.

Ashiralieva, A, Genersch, E. (2006). Reclassification, genotypes and virulence of Paenibacillus larvae, the etiological agent of American foulbrood in honeybees-a review. Apidologie 37, 411-420.

Bailey, L. (1960). The epizootiology of European foulbrood of the larval honey bee, Apis mellifera Linneaus. J. Insect. Pathol. 2, 67-83.

Bailey, L. (1963). The Pathogenicity for Honey-Bee Larvae of Microorganisms Associated with European Foulbrood. J. Insect. Pathol. 5, 198-205.

Bailey, L. (1983). Melissococcus pluton, the cause of European foulbrood of honey bees (Apis spp.). J. Appl. Microbiol. 55, 65-69.

Bailey, L., Ball, B. (1991). Honey Bee Pathology. London: Academic Press Limited.

Bailey, L., Locher, N. (1968). Experiments on the Etiology of European Foul Brood of the Honeybee. J. Apic. Res. 7, 103-107.

Barbosa, W. F., Smagghe, G., Guedes, R. N. C. (2015). Pesticides and reduced-risk insecticides, native bees and pantropical stingless bees: pitfalls and perspectives. Pest Manag. Sci. 71, 1049-53.

Belloy, L., Imdorf, A., Fries, I., Forsgren, E., Berthoud, H., et al. (2007). Spatial distribution of Melissococcus plutonius in adult honey bees collected from apiaries and colonies with and without symptoms of European foulbrood. Apidologie 38, 136140.

Blacquière, T., Smagghe, G., Van Gestel, C. A. M., Mommaerts, V. (2012). Neonicotinoids in bees: A review on concentrations, side-effects and risk assessment. Ecotoxicology 21, 973-992.

Bloodworth, B. C., Harn, C. S., Hock, C. T., Boon, Y. O. (1995). Liquid chromatographic determination of trans-10-hydroxy-2-decenoic acid content of commercial products containing royal jelly. J. AOAC Int. 78, 1019-1023. 
Buchmann, S. L., Repplier, B. (2005). Letters from the Hive. New York: Bantam Dell.

Bundesministerium für Ernährung und Landwirtschaft (2014). Bienen. Unverzichtbar für Natur und Erzeugung. Berlin. Verfügbar unter http://www.bmel.de/SharedDocs/Downloads/Broschueren/Bienen.pdf?_blob=public ationFile.

Cabrera-Marín, N. V, Liedo, P., Vandame, R., Sánchez, D. (2015). Foraging Allocation in the Honey Bee, Apis mellifera L. (Hymenoptera, Apidae), Tuned by the Presence of the Spinosad-Based Pesticide GF-120. Neotrop. Entomol. 44, 166-72.

Corby-Harris, V., Maes, P., Anderson, K. E. (2014a). The Bacterial Communities Associated with Honey Bee (Apis mellifera) Foragers. PLoS One 9, e95056.

Corby-Harris, V., Snyder, L. A., Schwan, M. R., Maes, P., McFrederick, Q. S., et al. (2014b). Origin and effect of Acetobacteraceae Alpha 2.2 in honey bee larvae and description of Parasaccharibacter apium, gen. nov., sp. nov. Appl. Environ. Microbiol. 80, 7460-72.

Engel, P., Kwong, W. K., Moran, N. A. (2013). Frischella perrara gen. nov., sp. nov., a gammaproteobacterium isolated from the gut of the honeybee, Apis mellifera. Int. J. Syst. Evol. Microbiol. 63, 3646-51.

Ferlat, S., Bottex-Gauthier, C., Picot, F., Potier, P., Vidal, D. (1994). Study of the immunomodulating properties of 10-hydroxy-2-decenoic acid [10-HDA], and its derivatives with glycerol, on a macrophage cell line. Trav. Sci. Cherch. Serv. Sante Armees, 161-162.

Forsgren, E. (2010). European foulbrood in honey bees. J. Invertebr. Pathol. 103 Suppl, S5-9.

Forsgren, E., Olofsson, T. C., Vásquez, A., Fries, I. (2009). Novel lactic acid bacteria inhibiting Paenibacillus larvae in honey bee larvae. Apidologie 41, 99-108.

Francisco, A. P., Vaz, C., Monteiro, P. T., Melo-Cristino, J., Ramirez, M., et al. (2012). PHYLOViZ: phylogenetic inference and data visualization for sequence based typing methods. BMC Bioinformatics 13, 87.

Gallai, N., Salles, J.-M., Settele, J., Vaissière, B. E. (2009). Economic valuation of the vulnerability of world agriculture confronted with pollinator decline. Ecol. Econ. 68, $810-821$.

Genç, M., Aslan, A. (1999). Determination of trans-10-hydroxy-2-decenoic acid content in pure royal jelly and royal jelly products by column liquid chromatography. $J$. Chromatogr. A 839, 265-268.

Genersch, E. (2008). Paenibacillus larvae and American Foulbrood - Long since known and still surprising. J. fur Verbraucherschutz und Leb. 3, 429-434.

Genersch, E. (2010a). American Foulbrood in honeybees and its causative agent, Paenibacillus larvae. J. Invertebr. Pathol. 103, S10-9. 
Genersch, E. (2010b). Honey bee pathology: current threats to honey bees and beekeeping. Appl. Microbiol. Biotechnol. 87, 87-97.

Genersch, E., Ashiralieva, A., Fries, I. (2005). Strain- and Genotype-Specific Differences in Virulence of Paenibacillus larvae subsp. larvae, a Bacterial Pathogen Causing American Foulbrood Disease in Honeybees. Appl. Environ. Microbiol. 71, 75517555.

Genersch, E., Forsgren, E., Pentikäinen, J., Ashiralieva, A., Rauch, S., et al. (2006). Reclassification of Paenibacillus larvae subsp. pulvifaciens and Paenibacillus larvae subsp. larvae as Paenibacillus larvae without subspecies differentiation. Int. J. Syst. Evol. Microbiol. 56, 501-11.

Genersch, E., von der Ohe, W., Kaatz, H., Schroeder, A., Otten, C., et al. (2010). The German bee monitoring project: a long term study to understand periodically high winter losses of honey bee colonies. Apidologie 41, 332-352.

Hasemann, L. (1961). How long can spores of American foulbrood live? Am. Bee J. 101, 298-299.

Haynes, E., Helgason, T., Young, J. P. W., Thwaites, R., Budge, G. E. (2013). A typing scheme for the honeybee pathogen Melissococcus plutonius allows detection of disease transmission events and a study of the distribution of variants. Environ. Microbiol. Rep. 5, 525-529.

Hegedus, D., Erlandson, M., Gillott, C., Toprak, U. (2009). New insights into peritrophic matrix synthesis, architecture, and function. Annu. Rev. Entomol. 54, 285-302.

Heyndrickx, M., Vandemeulebroecke, K., Hoste, B., Janssen, P., Kersters, K., et al. (1996). Reclassification of Paenibacillus (formerly Bacillus) pulvifaciens (Nakamura 1984) Ash et al. 1994, a later subjective synonym of Paenibacillus (formerly Bacillus) larvae (White 1906) Ash et al. 1994, as a subspecies of P. larvae, with emended descriptions of $P$. larvae as $P$. larvae subsp. larvae and $P$. larvae subsp. pulvifaciens. Int. J. Syst. Bacteriol. 46, 270-279.

Hroncova, Z., Havlik, J., Killer, J., Doskocil, I., Tyl, J., et al. (2015). Variation in Honey Bee Gut Microbial Diversity Affected by Ontogenetic Stage, Age and Geographic Location. PLoS One 10, e0118707.

Hulton, C. S., Higgins, C. F., Sharp, P. M. (1991). ERIC sequences: a novel family of repetitive elements in the genomes of Escherichia coli, Salmonella typhimurium and other enterobacteria. Mol. Microbiol. 5, 825-834.

Klein, A.-M., Vaissière, B. E., Cane, J. H., Steffan-Dewenter, I., Cunningham, S. A., et al. (2007). Importance of pollinators in changing landscapes for world crops. Proc. Biol. Sci. 274, 303-13.

Lindström, A., Korpela, S., Fries, I. (2008). The distribution of Paenibacillus larvae spores in adult bees and honey and larval mortality, following the addition of American foulbrood diseased brood or spore-contaminated honey in honey bee (Apis mellifera) colonies. J. Invertebr. Pathol. 99, 82-86. 
Martinson, V. G., Moy, J., Moran, N. A. (2012). Establishment of characteristic gut bacteria during development of the honeybee worker. Appl. Environ. Microbiol. 78, $2830-40$.

McKee, B. A., Djordjevic, S. P., Goodman, R. D., Hornitzky, M. A. (2003). The detection of Melissococcus pluton in honey bees (Apis mellifera) and their products using a hemi-nested PCR. Apidologie 34, 19-27.

Nagai, T., Inoue, R. (2004). Preparation and the functional properties of water extract and alkaline extract of royal jelly. Food Chem. 84, 181-186.

Olofsson, T. C., Vásquez, A. (2008). Detection and identification of a novel lactic acid bacterial flora within the honey stomach of the honeybee Apis mellifera. Curr. Microbiol. 57, 356-63.

Poppinga, L., Genersch, E. (2015). Molecular pathogenesis of American Foulbrood: how Paenibacillus larvae kills honey bee larvae. Curr. Opin. Insect Sci., 1-8.

Potts, S. G., Biesmeijer, J. C., Kremen, C., Neumann, P., Schweiger, O., Kunin, W. E. (2010). Global pollinator declines: trends, impacts and drivers. Trends Ecol. Evol. 25, 345-53.

Rauch, S., Ashiralieva, A., Hedtke, K., Genersch, E. (2009). Negative correlation between individual-insect-level virulence and colony-level virulence of Paenibacillus larvae, the etiological agent of American foulbrood of honeybees. Appl. Environ. Microbiol. $75,3344-7$.

Ritter, W. (2012). Bienen gesund erhalten. Stuttgart: Eugen Ulmer KG.

Rokop, Z. P., Horton, M. A., Newton, I. L. G. (2015). Interactions between co-occurring lactic acid bacteria in the honey bee hive. Appl. Environ. Microbiol. In Druck.

Sharples, G. J., Lloyd, R. G. (1990). A novel repeated DNA sequence located in the intergenic regions of bacterial chromosomes. Nucleic Acids Res. 18, 6503-6508.

Smith, K. M., Loh, E. H., Rostal, M. K., Zambrana-Torrelio, C. M., Mendiola, L., et al. (2014). Pathogens, Pests, and Economics: Drivers of Honey Bee Colony Declines and Losses. Ecohealth 10, 434-445.

Steffan-Dewenter, I., Potts, S. G., Packer, L., Ghazoul, J. (2005). Pollinator diversity and crop pollination services are at risk [3] (multiple letters). Trends Ecol. Evol. 20, 651653.

Sugiyama, T., Takahashi, K., Mori, H. (2012). Royal jelly acid, 10-hydroxy-trans-2decenoic acid, as a modulator of the innate immune responses. Endocr. Metab. Immune Disord. Drug Targets 12, 368-76.

Takamatsu, D., Arai, R., Miyoshi-Akiyama, T., Okumura, K., Okura, M., et al. (2013). Identification of mutations involved in the requirement of potassium for growth of typical Melissococcus plutonius strains. Appl. Environ. Microbiol. 79, 3882-3886. 
Takamatsu, D., Morinishi, K., Arai, R., Sakamoto, A., Okura, M., et al. (2014). Typing of Melissococcus plutonius isolated from European and Japanese honeybees suggests spread of sequence types across borders and between different Apis species. Vet. Microbiol. 171, 221-6.

Tautz, J. (2012). Phänomen Honigbiene. Berlin Heidelberg: Springer Spektrum.

Terra, W. R. (2001). The origin and functions of the insect peritrophic membrane and peritrophic gel. Arch. Insect Biochem. Physiol. 47, 47-61.

Vásquez, A., Forsgren, E., Fries, I., Paxton, R. J., Flaberg, E., et al. (2012). Symbionts as major modulators of insect health: lactic acid bacteria and honeybees. PLoS One 7, e33188.

Vásquez, A., Olofsson, T. C. (2009). The lactic acid bacteria involved in the production of bee pollen and bee bread. J. Apic. Res. 48, 189-195.

Viuda-Martos, M., Ruiz-Navajas, Y., Fernández-López, J., Pérez-Alvarez, J. A. (2008). Functional properties of honey, propolis, and royal jelly. J. Food Sci. 73, R117-24.

White, G. (1906). The bacteria of the apiary, with special reference to bee diseases. USDA, Bur. Entomol. Tech. Ser., 1-50.

White, G. (1912). The Cause of European Foulbrood. U.S. Dep. Agric. 157, 1-15.

Woodrow, A. W. (1942). Susceptibility of honeybee larvae to individual inoculations with spores of Bacillus larvae. J. Econ. Entomol. 35, 892-895.

Yue, D., Nordhoff, M., Wieler, L. H., Genersch, E. (2008). Fluorescence in situ hybridization (FISH) analysis of the interactions between honeybee larvae and Paenibacillus larvae, the causative agent of American foulbrood of honeybees (Apis mellifera). Environ. Microbiol. 10, 1612-20. 


\section{B Publikationen}




\title{
Die andere Faulbrut
}

\begin{abstract}
Marvin Djukic ${ }^{1}$ und Denise Hartken ${ }^{1}$
Deutsches Bienenjournal (2013), Vol. 8/2013, 17

${ }^{1}$ Genomische und Angewandte Mikrobiologie \& Göttingen Genomics Laboratory, Institut für Mikrobiologie und Genetik, Georg-August Universität Göttingen, Göttingen, Deutschland
\end{abstract}

\section{Anteilserklärung}

Manuskript/Minireview: MD, DH 


\section{Die andere Faulbrut}

Während in einigen Ländern Imker stark mit der Europäischen Faulbrut kämpfen, hört man in Deutschland wenig davon. Dennoch sollte man den Erreger auch hierzulande nicht aus den Augen verlieren.

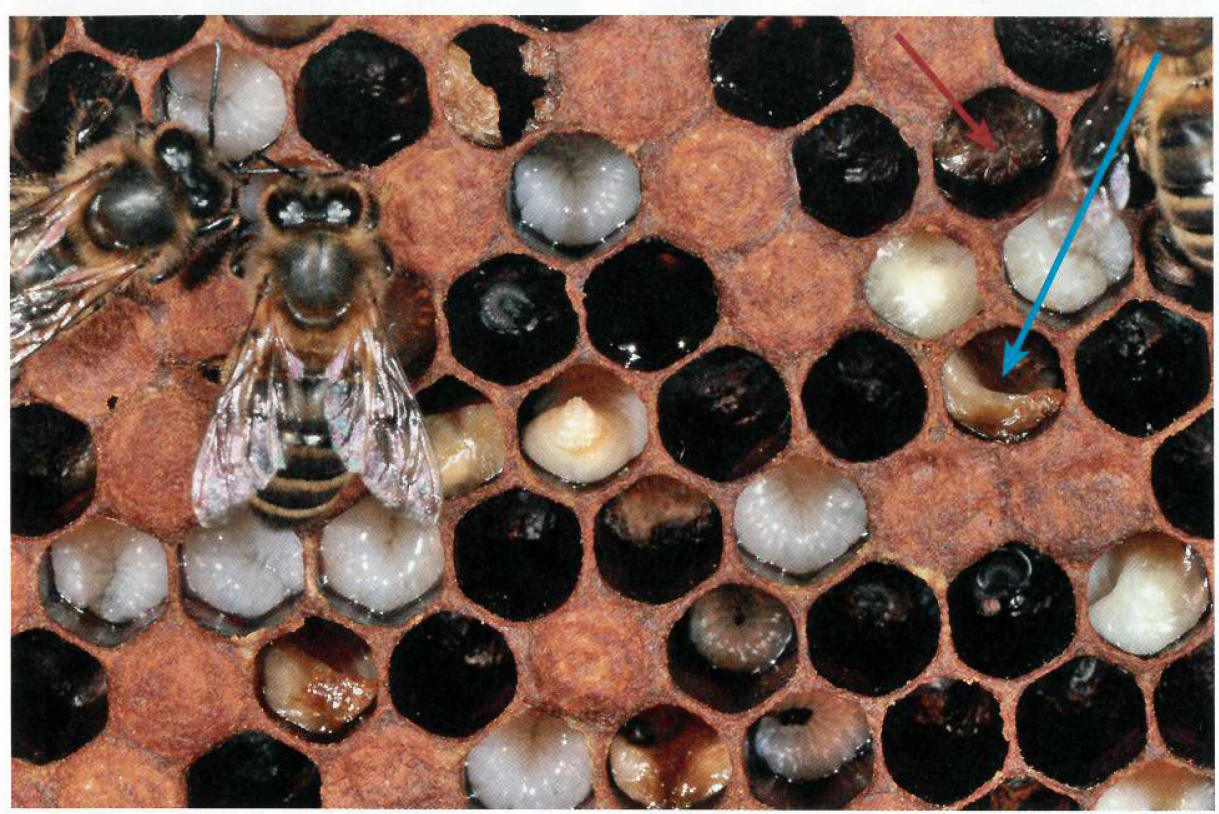

Larven dieser Brutwabe sind mit Europäischer Faulbrut befallen. Man erkennt eingetrocknete Brut (roter Pfeil) und verfärbte, verdrehte Maden (blauer Pfeil).

Foto: Kanton Bern

$\mathbf{E}$ uropäische Faulbrut, kurz EFB, ist wie Amerikanische Faulbrut (AFB) eine bakteriell verursachte Erkrankung der Bienenbrut. Doch genau wie die Bienen sind Bakterien erst in der Masse wirklich leistungsfähig. Allerdings sind nicht alle Bakterien schädlich. So können weder ausgewachsene Bienen noch deren Brut ohne Bakterien überleben. Beim Menschen ist die Darmflora gar zum Geschäft geworden - ein Beispiel sind die Laktobazillen in probiotischen Getränken.

Diese Milchsäurebakterien sollen bei Bienen auch die Erreger der AFB und der EFB hemmen. Im Gegensatz zu den ausgewach-

\section{Bitte um Meldung}

Für die Untersuchung des Erregers der EFB ist Marvin Djukic auf Ihre Mithilfe angewiesen. Wer vermutet, EFB am Stand zu haben, möge sich bitte bei inm melden. Djukic kommt dann gegebenenfalls zur vertraulichen und kostenlosen Probennahme vorbei. Sie erreichn inn telefonisch unter (05 51) 3933843 oder (0151) 12119728 sowie per E-Mail an mdjukic1@ gwdg.de. Weitere Infos finden Sie auf www.appmibio.uni-goettingen.de. senen Bienen besitzen die Larven jedoch noch keinen Honigmagen. Noch nicht einmal ein durchgängiger Darm ist vorhanden. Die Larven verfügen zudem nicht über dieselbe Darmflora wie erwachsene Bienen. Keime, wie Paenibacillus larvae (AFB) oder Melissococcus plutonius (EFB), setzen sich bereits einige Tagen nach der Eiablage gegen die sich noch entwickelnde Darmflora der Larve durch. Infiziert sich eine Larve, stirbt sie meist auch ab.

EFB erkennt man zuerst einmal daran, dass sich die Larve verfärbt. In der Regel stirbt die Brut noch im Rundmadenstadium ab. Dabei wechselt die Körperfarbe von Weiß über Gelb zu Braun. Es kann auch vorkommen, dass die Brut bereits verdeckelt ist, wenn sie abstirbt Dann sind oft Löcher in den Deckeln zu erkennen. Ein weiteres Indiz für eine Infektion mit dem Erreger ist die unnatürliche Position der Brut: Die Maden liegen meist seitlich nach oben verdreht in der Zelle. Der Geruch im Bienenstock kann, muss aber nicht, leicht säuerlich bis faulig sein. Das hängt meist davon ab, wie viel Brut durch die Krankheit bereits abgestorben ist.

Ein Erkennungsmerkmal der AFB ist die stark fadenziehende, braune Masse beim Streichholztest. Bei der EFB ist das etwas anders. Hier wird zwar auch gerne der Streich- holztest herangeführt, aber die tote Brut ist nicht immer fadenziehend. Oftmals vertrocknet die tote Larve im späteren Verlauf der Krankheit und wird dann von den Arbeiterinnen entfernt. Hier erkennt man einen wichtigen Unterschied: Eingetrocknete Brut ist einfacher zu entfernen als der braune Schleim, der bei der AFB entsteht - unabhängig von der Sporen- oder Kapselbildung des jeweiligen Erregers betrachtet. Aber Achtung: Die mit EFB infizierte, bereits leicht gelbliche Larve ist durchaus schleimig und fadenziehend, wenn man mit einem Streichholz darauf drückt und sie aufplatzt.

Es ist mir bislang kein effektives Mittel zur Vorbeugung einer EFB-Infektion bekannt. Damit Melissococcus plutonius wachsen kann, bedarf es nur einiger weniger Voraussetzungen. So müssen bestimmte Mineralien und Kohlenstoffe sowie eine gewisse Menge Wasser vorhanden sein. Der pH-Wert ist bei Bakterien als ein wichtiger Wachstumsfaktor beschrieben, wobei $M$. plutonius auch bei niedrigem pH-Wert wachsen kann. Temperatur und Atmosphäre - in diesem Fall die Kohlenstoffdioxidkonzentration - spielen ebenfalls eine Rolle. Ganz unabhängig vom Erreger diskutieren Imker und Wissenschaftler teils heftig darüber, inwieweit verschiedene Bienenrassen unterschiedlich anfällig für EFB-Infektionen sind. Grundsätzlich gilt aber, dass ein bereits vorerkranktes Volk anfälliger für EFB ist als ein gesundes.

In der Europäischen Union ist die Behandlung von Bienenvölkern mit Antibiotika nicht erlaubt. Von solch einer Behandlung ist ohnehin abzuraten, da sich Bakterien bei falscher und zu häufiger Anwendung der Antibiotika zügig anpassen und immun werden. Hingegen kann das Kunstschwarmverfahren zur Sanierung des Bienenstandes durchaus in Betracht gezogen werden.

\section{DIE AUTOREN}
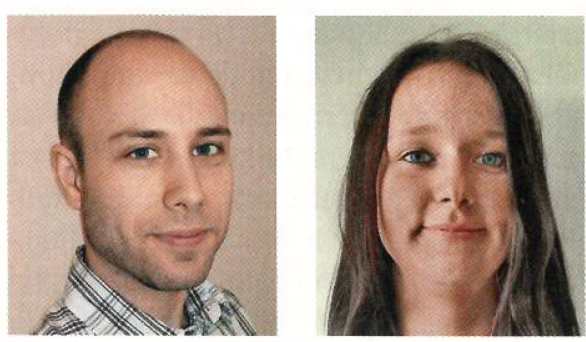

\section{Marvin Djukic und Denise Hartken}

arbeiten an der Georg-August-Universität Göttingen. Djukic untersucht im Rahmen einer Doktorarbeit Amerikanische und Europäische Faulbrut. Schwerpunkt sind die Genomanalyse der Krankheitserreger und die Ermittlung von potenziellen Virulenzmechanismen. Hartken erforscht als Bachelorstudentin die Europäische Faulbrut und deren Sekundärerreger. 


\title{
How to Kill the Honey Bee Larva: Genomic Potential and Virulence Mechanisms of Paenibacillus larvae
}

\author{
Marvin Djukic $^{1 *}$, Elzbieta Brzuszkiewicz ${ }^{1 *}$, Anne Fünfhaus ${ }^{2}$, Jörn Voss ${ }^{1}$, Kathleen \\ Gollnow $^{1}$, Lena Poppinga ${ }^{2}$, Heiko Liesegang ${ }^{1}$, Eva Garcia-Gonzalez ${ }^{2}$, Elke Genersch ${ }^{2}$ und \\ Rolf Daniel ${ }^{1}$
}

PLoS ONE (2014), Vol. 9, e90914

\footnotetext{
${ }^{1}$ Abteilung für Genomische und Angewandte Mikrobiologie \& Göttingen Genomics Laboratory, Institut für Mikrobiologie und Genetik, Georg-August Universität Göttingen, Göttingen, Deutschland

${ }^{2}$ Abteilung für Molekulare Mikrobiologie und Bienenkrankheiten, Länderinstitut für Bienenkunde, Hohen Neuendorf, Deutschland
}

\section{Anteilserklärung}

* MD und EB trugen zu gleichen Teilen bei.

Idee/Konzept: EB, EG, RD

Durchführung der Experimente: MD, EB, AF, KG, LP, EGG

Datenauswertung: MD, EB, AF, JV, LP, HL, EGG, EG

Schreiben des Manuskripts: MD, EB, EG, RD 


\title{
How to Kill the Honey Bee Larva: Genomic Potential and Virulence Mechanisms of Paenibacillus larvae
}

\author{
Marvin Djukic ${ }^{19}$, Elzbieta Brzuszkiewicz ${ }^{19}$, Anne Fünfhaus ${ }^{2}$, Jörn Voss ${ }^{1}$, Kathleen Gollnow ${ }^{1}$, \\ Lena Poppinga ${ }^{2}$, Heiko Liesegang ${ }^{1}$, Eva Garcia-Gonzalez ${ }^{2}$, Elke Genersch ${ }^{2 *}$, Rolf Daniel ${ }^{1 *}$
}

1 Department of Genomic and Applied Microbiology and Göttingen Genomics Laboratory, Institute of Microbiology and Genetics, Georg-August-University Göttingen, Göttingen, Germany, 2 Department for Molecular Microbiology and Bee Diseases, Institute for Bee Research, Hohen Neuendorf, Germany

\begin{abstract}
Paenibacillus larvae, a Gram positive bacterial pathogen, causes American Foulbrood (AFB), which is the most serious infectious disease of honey bees. In order to investigate the genomic potential of $P$. larvae, two strains belonging to two different genotypes were sequenced and used for comparative genome analysis. The complete genome sequence of $P$. larvae strain DSM 25430 (genotype ERIC II) consisted of 4,056,006 bp and harbored 3,928 predicted protein-encoding genes. The draft genome sequence of $P$. larvae strain DSM 25719 (genotype ERIC I) comprised 4,579,589 bp and contained 4,868 protein-encoding genes. Both strains harbored a $9.7 \mathrm{~kb}$ plasmid and encoded a large number of virulence-associated proteins such as toxins and collagenases. In addition, genes encoding large multimodular enzymes producing nonribosomally peptides or polyketides were identified. In the genome of strain DSM 25719 seven toxin associated loci were identified and analyzed. Five of them encoded putatively functional toxins. The genome of strain DSM 25430 harbored several toxin loci that showed similarity to corresponding loci in the genome of strain DSM 25719, but were non-functional due to point mutations or disruption by transposases. Although both strains cause AFB, significant differences between the genomes were observed including genome size, number and composition of transposases, insertion elements, predicted phage regions, and strain-specific island-like regions. Transposases, integrases and recombinases are important drivers for genome plasticity. A total of 390 and 273 mobile elements were found in strain DSM 25430 and strain DSM 25719 , respectively. Comparative genomics of both strains revealed acquisition of virulence factors by horizontal gene transfer and provided insights into evolution and pathogenicity.
\end{abstract}

Citation: Djukic M, Brzuszkiewicz E, Fünfhaus A, Voss J, Gollnow K, et al. (2014) How to Kill the Honey Bee Larva: Genomic Potential and Virulence Mechanisms of Paenibacillus larvae. PLoS ONE 9(3): e90914. doi:10.1371/journal.pone.0090914

Editor: Stefan Bereswill, Charité-University Medicine Berlin, Germany

Received December 28, 2013; Accepted February 5, 2014; Published March 5, 2014

Copyright: (c) 2014 Djukic et al. This is an open-access article distributed under the terms of the Creative Commons Attribution License, which permits unrestricted use, distribution, and reproduction in any medium, provided the original author and source are credited.

Funding: EG was supported by grants from the Ministries for Agriculture from Brandenburg and Sachsen-Anhalt, Germany. AF and LP were supported by the German Research Foundation (DFG, grant GE 1365/1-1). EG-G was supported by the German Research Foundation (DFG, Graduate School 1121). The Göttingen group was supported by grants from the Niedersächsisches Ministerium für Wissenschaft und Kultur and the Bundesministerium für Bildung und Forschung. The funders had no role in study design, data collection, data analysis, data interpretation, writing of the report; and the decision to submit the article for publication.

Competing Interests: The authors have declared that no competing interests exist.

*E-mail: rdaniel@gwdg.de (RD); elke.genersch@rz.hu-berlin.de (EG)

9 These authors contributed equally to this work.

\section{Introduction}

Honey bees (Apis mellifera) are among the most important livestock due to their role in pollination of many crops, fruits, and wild flowers [1]. Nowadays, $90 \%$ of commercial pollination is performed by managed honey bees and the demand for this service is growing faster than the global stock of honey bees [2,3]. This might lead to an imbalance of supply and demand in the near future. Therefore, honey bee health is of crucial importance not only for apiculture but also for agriculture and human food security.

Honey bees are attacked by numerous pathogens and parasites including viruses, bacteria, fungi, and metazoans [4]. Paenibacillus larvae is one of the two bacterial species known to be pathogenic for honey bees. This Gram-positive, spore-forming and peritrichously flagellated bacterium is the causative agent of American Foulbrood (AFB) [5], a fatal, globally spread epizootic disease. Although AFB only kills infected honey bee larvae, it eventually leads to the collapse of entire colonies when left untreated. AFB is also considered very contagious; therefore, it is a notifiable disease in most countries.

The spores of $P$. larvae are the infectious form. Larvae are most susceptible to infection during the first 36 hours after egg hatching when a few spores per larva are sufficient to initiate infection; at later larval developmental stages spore doses needed to successfully infect a larva are too high to occur under natural conditions $[6,7]$. Soon after ingestion, spores germinate in the larval midgut, where they massively proliferate for several days without destroying the integrity of the midgut epithelium [8]. At a later stage of infection, $P$. larvae breaches the peritrophic matrix [9] and the epithelial barrier and invades the haemocoel. Recent studies revealed that $P$. larvae destroys cell-cell and cell-matrix junctional structures to follow the paracellular route from the gut lumen into larval tissue. Breaching of the epithelium was shown to coincide with larval death [8].

The species $P$. larvae comprises four different genotypes named ERIC I to ERIC IV $[5,10]$. All four genotypes differ in several phenotypic characteristics $[5,11,12]$, most importantly in virulence $[7,13]$. Epidemiological studies showed that only ERIC I and II 
are frequently isolated from AFB-diseased colonies [14-17]. Thus, ERIC I and II are the most important genotypes with respect to infection of honey bee larvae. The genotype-specific differences in virulence between $P$. larvae ERIC I and II correspond to the time it takes to kill infected larvae [5,7]. Members of ERIC II are rather fast killers with an $\mathrm{LT}_{100}$ of approximately seven days while members of ERIC I are killing more slowly $\left(\mathrm{LT}_{100}\right.$ approximately 12 days) [18]. These differences in virulence on the individual larval level also influence the virulence on the colony level [14].

Our knowledge on $P$. larvae and the pathogenesis of AFB increased tremendously over the past decade [19]. Two draft genome sequences of two $P$. larvae strains are available [20,21], but with large numbers of remaining gaps. In addition, several putative virulence factor genes are differentially present in the genomes of the four ERIC-genotypes of $P$. larvae [5]. However, most molecular aspects of this important pathogen still remain elusive.

Here, we present the whole genome sequences of $P$. larvae genotypes ERIC I (strain DSM 25719) and ERIC II (strain DSM 25430) and a comparative analysis to elucidate both, the general pathogenic mechanisms of $P$. larvae and the genotypic differences in virulence. The study is focused on the identification of potential virulence genes in each genome and analysis of genotype-specific differences between the two P. larvae genotypes ERIC I and ERIC II.

\section{Results and Discussion}

\section{General Genomic Features}

We have sequenced, manually curated and annotated the genomes of two $P$. larvae isolates representing the two genotypes ERIC I (strain DSM 25719) and ERIC II (strain DSM 25430). The general features of both genomes are presented in Table 1. The complete genome of strain DSM 25719 consisted of $4,579,589$ bp whereas the one of strain DSM 25430 harbored 4,056,006 bp. The number of replicons was identical in both strains. A total of 4,868 and 3,928 protein-encoding genes were predicted for DSM 25719 and DSM 25430, respectively. The higher overall genome size of strain DSM 25719 is mainly due to the presence of additional prophage regions (Table 1). We could identify 8 putatively phage-related regions within the DSM 25430 genome. However, all of them appeared incomplete. Within the DSM 25719 genome 22 phage-related regions have been identified. (Figure 1, Table 1) [22].

IS elements are transposable DNA fragments that provide the structural basis for rearrangements of genomic fragments, incorporation of foreign DNA into the genome, and homologous recombination [23,24]. We found 390 mobile elements (transposases, integrases and recombinases) in the genome of DSM 25430 , and 273 in that of DSM 25719 (Table 1). A striking difference of both strains is the high copy number of mutator-type transposases in the DSM 25430 genome. Nevertheless, the large number of mobile genetic elements and prophage regions in the genomes of both $P$. larvae strains suggested frequent genome rearrangements and a high degree of genome plasticity.

This feature of the $P$. larvae genome has also been recognized recently, when Chan and co-workers tried an update and draft annotation of the $P$. larvae sequence [21] originally published by Qin and co-workers [20]. The number of contigs could be reduced from 646 [20] to 388 [21] but still no complete genome sequence could be obtained. The annotation even based on the original 646 contig-version. The authors hypothesized that the fragmentation of their assembly may be due to long genomic repeats that could not be bridged by their sequencing strategy. Our data confirms the existence of genomic regions containing repeats and repetitive sequences, which indeed were difficult but not impossible to sequence. We were able to close the sequence of the DSM 25430 replicons, which are now available without any gap. The final sequence of the DSM 25719 strain consists of only seven contigs with length 3,663,994 bp, 771,602 bp, 86,545 bp, 77,837 bp, $12,832 \mathrm{bp}, 8,981 \mathrm{bp}$ and $8,080 \mathrm{bp}$.

In both strains we found a $9.7 \mathrm{~kb}$-circular plasmid designated pPLAl_10 in DSM 25719 and pPLA2_10 in DSM 25430 with almost identical sequences differing in only 49 bases (Figure S1). A gene encoding a putative replication initiation factor (REP) was identified in both replicons. A plasmid of similar size (pPl19.4) has been reported for ERIC II-strains [12]. The existence of a plasmid in strains of $P$. larvae ERIC I was contradictory to an earlier report in which pPll9.4 was found exclusively in ERIC II-strains and, therefore, had been considered as characteristic for ERIC II [12]. Screening an international collection of 65 ERIC I strains and 30 ERIC II strains revealed that indeed no other strains of the ERIC I genotype harbored the pPLAl_10 plasmid indicating that the one found in DSM 25719 was strain-specific but not genotypespecific.

\section{Metabolism}

Energy metabolism. $P$. larvae is a facultative anaerobic organism that grows preferentially under aerobic conditions. In addition to typical oxygen-dependent respiration, both strains are able to utilize nitrate as alternative electron acceptor. Genes encoding a putative respiratory nitrate reductase (NarGHI; ERIC1_1c24810 -ERIC1_1c24830, ERIC2_c40560 ERIC2_c40590) are present in the genomes of both strains but complete general nitrite reductase genes could be found only in genome of DSM 25430 (NasDE; ERIC2_c25390 ERIC2_c25400).

Sugar metabolism. At the beginning of the infectious process, during the non-invasive phase $P$. larvae proliferates in the midgut of the larvae [8] and lives on the incoming larval diet. The diet of worker and drone larvae changes over time from pure royal jelly (RJ) to a mixture of RJ, honey, and pollen while queen larvae are fed RJ throughout their entire larval development [25]. In any case, crude proteins (12.5\% in RJ) [26] and simple sugars (11\% in RJ) [26] are the main constituents of the larval diet. Fructose and glucose, which are the dominant sugars of the added honey, can be metabolized by vegetative $P$. larvae [12]. Our genome analysis revealed that $P$. larvae metabolizes $\mathrm{D}$-glucose and D-fructose mainly via the Embden-Meyerhof-Parnas (EMP) and oxidative pentose-phosphate pathway, as a complete set of genes for the conversion of glucose 6-phosphate to pyruvate was present and genes coding for a 1-phoshofructokinase (ERIC1_1c31490; ERIC2_c36850) and 6-phosphofructokinase (ERIC1_1c16820; ERIC2_c11750) were found. The genomes of both sequenced strains also revealed a possible mechanism for the uptake and catabolism of trehalose, the main carbohydrate in honey bee larval hemolymph [27]. Both strains are equipped with a putative trehalose specific $\mathrm{II}^{\mathrm{c}}$ component of a PTS system (ERIC1_1c04630; ERIC2_c17680) for uptake of the disaccharide. A regulated uptake system for trehalose might correspond to the second, the invasive phase of infection. In this phase when the bacteria invade the haemocoel [8] and start to consume and degrade the entire larval biomass, disaccharides like trehalose become available.

\section{Toxins}

Bacterial pathogens need a diverse repertoire of genes providing them with unique mechanisms to colonize the host and escape the 

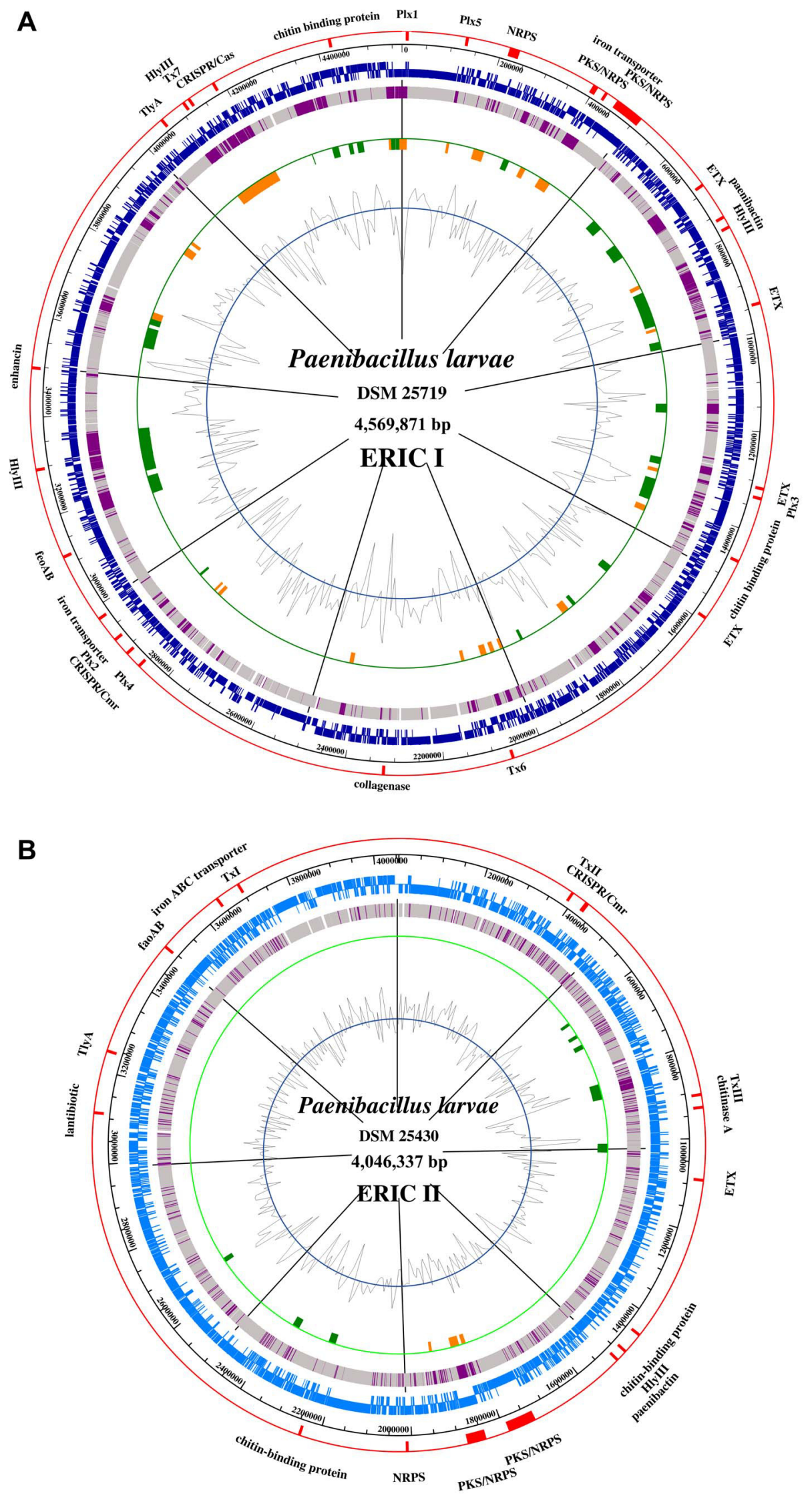

Figure 1. Maps of the P. larvae DSM 25719 (A) and DSM 25430 (B) chromosome The different circles represent (from inside): (a), GC content; (b), strain-specific regions (orange) and prophages (green); (c), genes present in both analyzed strains (grey) and genes 
found only in one strain (purple); (d), all ORFs clockwise and anticlockwise or (blue); (e), scale; (g), highlighted genes, gene clusters, including toxins, potential virulence factors mentioned in the manuscript (red).

doi:10.1371/journal.pone.0090914.g001

host's immune system. These genes and the corresponding gene products conferring the pathogenic phenotype can be summarized as virulence genes and virulence factors, respectively. Potential virulence-associated determinants of both $P$. larvae genotypes ERIC I and ERIC II were identified in silico based on sequence similarity to known microbial virulence factors.

AB Toxin loci. It was recently demonstrated that breaching of the larval midgut epithelium and invasion of the haemocoel is a crucial step in $P$. larvae pathogenesis. It was suggested that the observed changes in epithelial cell morphology during this process are the result of toxin activity [8]. In accordance, putative $A B$ toxin gene fragments have been identified in ERIC I strains by subtractive suppression hybridization [28]. This result could recently be supported during the draft annotation of the 646 contigs of the fragmented $P$. larvae genome sequence. The existence of allegedly sixteen toxin proteins was suggested [20,21]. Here, we present the definite identification of seven toxin encoding loci (Plx1-7) in the genome of ERIC I genotype strain DSM 25719 (Figure 2) with five of them coding for putatively functional toxins (Plx1-5) (Figure 2). All five putatively functional gene products show similarity to the family of $\mathrm{AB}$ toxins known from several other Gram positive and spore-forming bacteria such as pathogenic clostridia and bacilli. $\mathrm{AB}$ toxins consist of two subunits (A and B) and display a synergistic binary mechanism for attacking eukaryotic cells. The A subunit possesses enzyme activity and inhibits normal cell functions. The B subunit mediates membrane-binding and transport of the A domain into the host cell (for a recent review: [29]). Based on comparative genome analysis via suppression subtractive hybridization [28] and the application of recently developed molecular tools for $P$. larvae [30], two of the five putatively functional $P$. larvae toxins, $\mathrm{Plx} 1$ and $\mathrm{Plx} 2$, have already been characterized in detail [31]. Plxl (Accession No. KC456421; ERIC1_1c00040) is a single-chain AB toxin and belongs to an enigmatic family of toxins [31], so far comprising only few members, the larvicidal toxin MTX1 expressed by Lysinibacillus sphaericus and several pierisin-like toxins expressed by Pieridae, a large family of butterflies [32,33]. It has been proposed that Plxl has ADP ribosyltransferase activity [31] as already shown for MTX1 and pierisin-1 [32,33]. Plx2 was shown to be a binary $\mathrm{AB}$ toxin with two separate ORFs encoding the $\mathrm{A}$ and $\mathrm{B}$ subunits [31]. The A subunit (Plx2A, ERIC1_1c30800) showed similarity to C3-like Rho-ADP-ribosylating toxins whereas the B subunit (Plx2B, ERIC1_1c30790) showed similarity to the B subunit of the C2 binary toxin of Clostridium botulinum [31]. Exposure bioassays performed with wildtype $P$. larvae and corresponding knock-out mutants lacking Plx1 or Plx2 expression revealed that both toxins are important virulence factors for $P$. larvae ERIC I [31].

Two other toxin loci in the genome of $P$. larvae ERIC I (Plx4, and Plx5) also encode binary $\mathrm{AB}$ toxins with separate ORFs coding for "A" domains and "B" domains. The "B" domains (ERIC1_1c29950, ERIC1_1c01270) are located upstream of the "A"-domains (ERIC1_1c29960, ERIC1_1c01280). The two predicted "A" domains show similarity to ADP ribosyltransferases of B. cereus or B. thuringiensis whereas the predicted "B" domains show similarity to the "B" domains of clostridial enterotoxic C2 toxin of Clostridium botulinum or toxin CDT of $C$. difficile (for a recent review on binary toxins see [29]). The combination of Clostridiumlike translocation domains with $B$. cereus-like ADP ribosyltranferases is unique and indicates that $P$. larvae developed its own specific mechanisms to intoxicate honey bee larval cells.

Two P. larvae ERIC I toxin loci (Tx6, Tx7) harbor only remnants of toxin genes. In $\mathrm{T} x 6$, a putative $\mathrm{B}$ domain gene is interrupted by three mutations splitting the gene into several ORFs (ERIC1_1c21800 to ERIC1_1c21770); the upstream located putative A domain gene is interrupted by one mutation resulting in two ORFs (ERIC1_1c21750 - ERIC1_1c21740). In addition, a transposase is inserted between the genes encoding $\mathrm{B}$ and $\mathrm{A}$ domain. These mutations indicate that the $\mathrm{AB}$ toxin gene cluster is non-functional. Downstream of this cluster, remnants of a Clostridium botulinum neurotoxin type A gene cluster [34] are located (Figure 3). ORF X2, ORF X3 (interrupted by a stop codon), and p47 are present whereas ORF X1, the botulinum neurotoxin and ntnH genes are missing (Figure 3). The existence of these genes suggests horizontal gene transfer from the food-borne pathogen $C$. botulinum to $P$. larvae. Correspondingly, $C$. botulinum can frequently

Table 1. General genomic features of the P. larvae strains.

\begin{tabular}{|c|c|c|}
\hline Feature & DSM 25719 (ERIC I) & DSM 25430 (ERIC II) \\
\hline Status & 8 contigs & 2 contigs \\
\hline Chromosome size & $4,569,871$ bp (7 contigs) & 4,046,337 bp (closed) \\
\hline Plasmid size & 9,718 bp (closed) & 9,669 bp (closed) \\
\hline GC content & $44 \%$ & $45 \%$ \\
\hline No. rRNA genes & 23 & 25 \\
\hline No. tRNA genes & 79 & 81 \\
\hline No. ORFs & 4,868 & 3,928 \\
\hline No. pseudogenes & 75 & 99 \\
\hline No. transposases & 256 & 366 \\
\hline No. phage integrases and site-specific recombinases & 17 & 24 \\
\hline No. phage regions & 22 & 8 \\
\hline Phage regions size & 693.4 kbp & 153.4 kbp \\
\hline
\end{tabular}




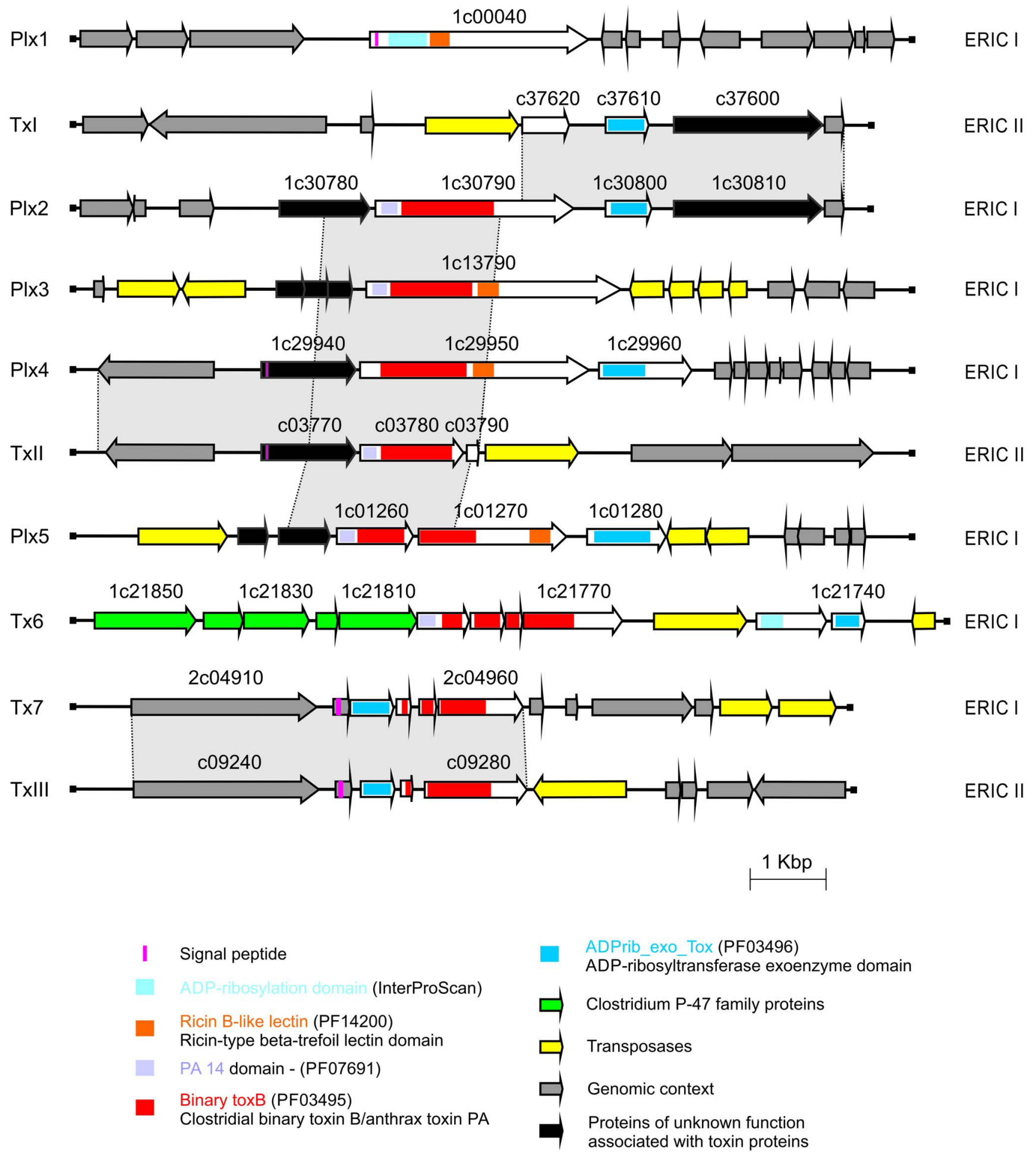

Figure 2. Genetic organization of toxin complex loci identified in $P$. larvae DSM 25719 and DSM 25430. Related ORFs are shown in the same colors. Toxin loci PIx1-7 are encoded within P. larvae DSM 25719 genome (ERIC I) and toxin loci TxI-III within P. larvae DSM 25430 (ERIC II). doi:10.1371/journal.pone.0090914.g002

be found in the beehive environment and especially in wax and honey $[35,36]$. One explanation for the loss of function in $P$. larvae is that the neurotoxin type A gene cluster coding for a vertebrate toxin may not confer any fitness increase for entomopathogenic $P$. larvae. In $\mathrm{Tx} 7$, a $\mathrm{B}$ domain gene, which is interrupted by two mutations resulting in three $\mathrm{ORF}_{\mathrm{s}}$, is located upstream of a putative gene encoding an A domain. Although the putative A domain gene appears functional, it is unlikely that this gene cluster codes for a functional $\mathrm{AB}$ toxin. However, the formation of functional toxin complexes involving the Tx7 A domain might still be possible by interaction of the $\mathrm{A}$ domain with a $\mathrm{B}$ domain encoded by another toxin locus. 


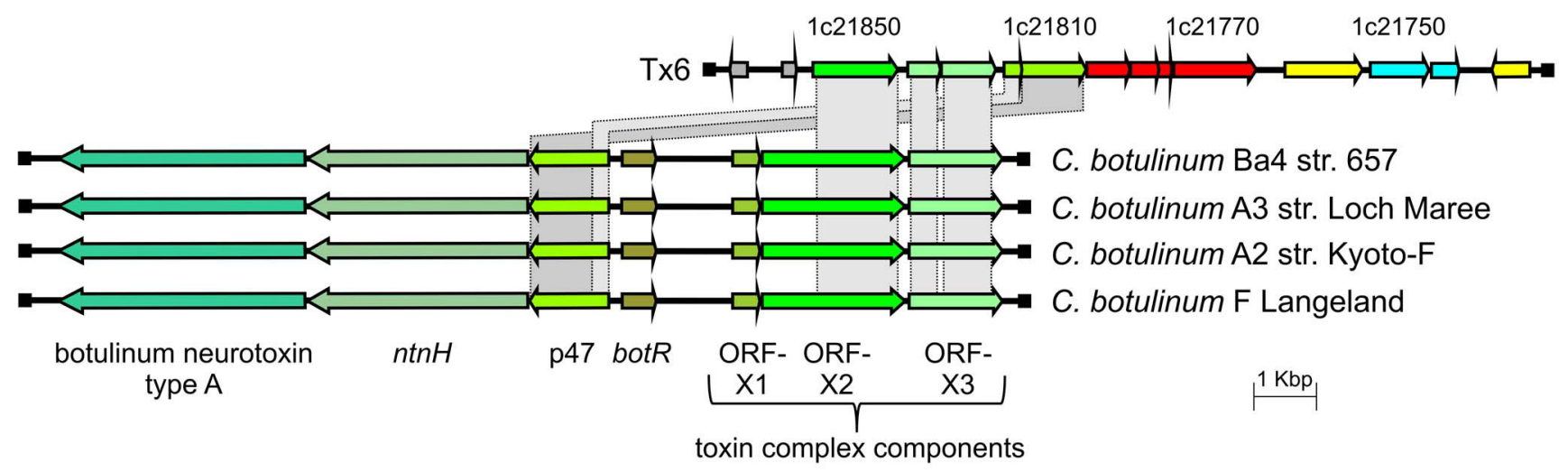

Figure 3. Comparison of the $P$. larvae DSM 25719 Tx6 toxin-encoding locus with selected strains. Related ORFs are shown in the same following colors: yellow, transposases; grey, genomic context; green, botulinum toxin complex components; cyan, binary toxin A domain-containing protein; and red, binary toxin B domain-containing proteins. The arrows indicate the direction of transcription. doi:10.1371/journal.pone.0090914.g003

In the genome of $P$. larvae DSM 25430 (genotype ERIC II), we identified five toxin-encoding loci and none of them harbored a putatively functional toxin gene. Sequence comparison revealed that TxI, TxII, and TxIII of DSM 25430 are remnants of Plx2, Plx4, and Tx7 loci of DSM 25719 (Figure 2). Additionally, DSM 25430 encodes small fragments of toxins (ERIC2_c05300, ERIC2_c05310, ERIC2_c33880). Several mutations including insertion of transposases and deletions of gene fragments in DSM 25430 resulted in toxin loci, which only harbor footprints of formerly functional toxin gene clusters. Thus, although highly virulent on individual larval level [7], strain DSM 25430 lacked classical toxins raising the interesting question of how this bacterium is killing honey bee larvae.

Many entomopathogenic bacteria like B. thuringiensis, Brevibacillus laterosporus and Lysinibacillus sphaericus produce large crystalline inclusions that consist of entomocidal protein protoxins [37]. These parasporal crystal proteins (also named cry-toxins or $\delta$ endotoxins) are activated upon ingestion through proteolytic processing in the midgut environment. Subsequently, pore structures capable of inserting into the host cell membrane and eventually leading to cell death are formed. Although P. larvae is an acrystalliferous bacterium, which does not produce $\delta$-endotoxins, four toxin loci in strain DSM 25719 and DSM 25430 contain short ORFs with weak similarity to $\delta$-endotoxins of $B$. thuringiensis. These ORFs are located downstream of the genes coding for the putative "AB" toxins $\mathrm{Plx} 2, \mathrm{Plx} 3, \mathrm{Plx} 4$, and $\mathrm{Plx} 5$ and the B domain gene in TxIII. The role of these ORFs is unclear, as they are too short to code for functional cry-like toxins and some of them are interrupted by insertions/deletions and/or frameshift mutations.

We also identified four clostridial epsilon toxin ETX/Bacillus mosquitocidal toxin MTX2 gene homologs in the genome of DSM 25719 (ERIC1_1c06220, ERIC1_1c16820, ERIC1_1c13570, ERIC1_1c09720). Comparison of the predicted amino acid sequences revealed $31 \%$ identity to epsilon toxin type B of Clostridium perfringens (Q02307) and 29\% identity to a novel mosquitocidal toxin Mtx2 encoded by L. sphaericus (Accession Q45422). Mtx2 is unrelated to AB toxin MTX1 but shares regions of similarity with epsilon toxin type $\mathrm{B}$ of $C$. perfringens. Epsilon toxin is the major virulence factor of $C$. perfringens types $\mathrm{B}$ and $\mathrm{D}$. This microorganism is responsible for fatal enterotoxaemia in animals, mainly in lambs and goat, and more rarely in cattle [38]. The functionality and role of these putative epsilon toxin homologs in $P$. larvae DSM 25719 need to be established.
In $P$. larvae DSM 25430, one ORF (ERIC2 c11360) corresponding to the Mtx2 homolog (ERIC1_1C06220) of DSM 25719 could be identified. However, insertion of a transposase at the $5^{\prime}$ end destroyed the $5^{\prime}$-region of the gene including the start codon. Therefore, it is unlikely that a functional epsilon toxin homolog exists in strain DSM 25430.

In conclusion, we identified several putative functional toxinencoding genes and gene clusters in the genome of DSM 25719, but none in the genome of DSM 25430. The genome of DSM 25430 harbored several toxin loci that show similarity to corresponding loci in the genome of DSM 25719, but these were non-functional. These results confirmed previous findings obtained by subtractive suppression hybridization $(\mathrm{SSH})$ that failed to identify any toxin gene in P. larvae genotype ERIC II but already suggested the existence of AB toxins in ERIC I [28]. These results are surprising, since ERIC II is more virulent than ERIC I on the larval level and kills larvae faster than ERIC I [5]. This tremendous difference between the two genotypes indicates that they developed completely different modes of pathogenesis or the genes, which encode the lethal factors for the honey bee have not been identified yet. While ERIC I might still rely on toxins for killing larvae, ERIC II became independent from toxins most likely by acquiring other virulence factors such as the recently identified ERIC II-specific S layer protein SplA [30].

Cytolysins, iron acquisition. The genomes of $P$. larvae DSM 25719 and DSM 25430 harbored four (ERIC1_1c07340, ERIC1_2c04780, ERIC1_1c35160, ERIC1_2c04240) and two (ERIC2_c15500, ERIC2_c32790) genes, which were predicted by similarity-based annotation as hemolysin domains. Hemolysins, or more correctly cytolysins, are a group of membrane-damaging toxins that disrupt host cell membranes either enzymatically (by the means of proteases or phospholipases) or by forming pores in the host cell membrane (pore-forming toxins, PFTs). However, the highest similarity of the proteins ERIC1_1c07340, ERIC1_2c04780, ERIC1_1c35160 and ERIC2_c15500 point to the hemolysin III-related protein family whereas ERIC1_2c04240 and ERIC2_c32790 cluster within the pore-forming cytolysin TlyA protein family. Apparently these cytolysins represent members of a new Paenibacillus-specific protein family and may play an important role in bacterial pathogenesis. It has been reported that the related cytolysins directly act on certain mammalian cells as cytotoxic virulence factors or are responsible for iron acquisition during bacterial growth. It has been shown that hemolysin III of $B$. cereus is a pore-forming, haemolytic 
cytolysin [39]. Iron acquisition during growth in mammalian as well as insect hosts poses specific difficulties for bacterial pathogens and thus might be the reason for the acquisition of related toxin genes.

The availability of iron within extracellular fluid is highly restricted. Bacteria living inside their hosts often need to establish methods for extracting the metal from host proteins. Iron chelators (siderophores) are produced and secreted specifically in response to iron deficiency. The genome of $P$. larvae DSM 25719 encodes two iron ABC transport systems (ERIC1_1c03900-ERIC1_1c03960 and ERIC1_1c31350-ERIC1_1c31370) whereas DSM 25430 encodes only one (ERIC2_c36980-ERIC2_c37000). The gene ERIC1_1c03900 showed similarity to the iron-regulated surface determinant (isd) system, which is used by Staphylococci to bind hemoproteins, remove the heme molecule, and transport heme into the bacterial cytoplasm. Its function in $P$. larvae during larval infection needs to be established. Additionally, both strains contain genes for a ferrous iron transport cluster, which encode a FeoA family protein (ERIC1_1c32860 and ERIC2_c35360) and the ferrous iron uptake protein B (ERIC1_1c32870, ERIC2_c35350).

\section{Proteases, Collagenases, Chitinases}

Proteases have been discussed as key virulence factors of $P$. larvae since decades [16,40,41]. We found 159 full or truncated proteases in the genome of DSM 25719 and 128 in that of DSM 25430, which belong to different families (Table S1, Table S2) [42]. Some of these enzymes might be involved in disruption of the epithelial barrier integrity of honey bee larvae by degrading cell-cell and cell-matrix junctional structures.

The main structural component of the extracellular matrix is collagen. To destroy this barrier bacteria secrete enzymes degrading the major matrix components such as collagenases, hyaluronidases and proteases [43]. Collagenases have been widely used for the disintegration of connective tissue and separation of tissue culture cells, because of the broad substrate specificity [44]. We identified putative genes encoding collagenases, which belong to two different families (Table S1, Table S2). Microbial collagenases family 9 have been identified from bacteria belonging to the genera Vibrio and Clostridium [45,46]. Collagenase is used to degrade the collagen barrier of the host during invasion. Peptidases family M9 (ERIC1_1c24570, ERIC2_c40370), and peptidases belonging to family U32 (ERICi_1c37420, ERIC1_1c37430, ERIC2_c05010, ERIC2_c27620, and ERIC2_c27630) were identified in both genomes. Glycosaminoglycans, like hyaluronan and chondroitin, are polymers built of hexosamine uronic acid disaccharide units and are a major component of the extracellular matrix [47]. We identified one putative polysaccharide lyase family 8 protein in each genome (ERIC1_1c09810, ERIC2_c23740). Polysaccharide lyase family 8 consists of a group of secreted bacterial lyase enzymes, e.g. hyaluronidases, which are able to degrade hyaluronan, chondroitin, and chondroitin sulfates [48]. Hyaluronidases are also known as virulence factors [49], as they are able to degrade the connective tissue of eukaryotes [50].

Chitinases. The peritrophic matrix (PM) represents the first barrier for the bacteria to reach the epithelium [9]. The PM is a chitin and glycoprotein layer that borders the larval midgut and protects the midgut epithelium from abrasive food particles, digestive enzymes and pathogen infections [51]. In both analyzed genomes, we found genes with chitin-binding domains (ERIC1_3c00760, ERIC1_1c15380; ERIC2_c22220 and ERIC2_c15060), which might aid in chitin degradation. Additionally, a region splitted in several ORFs and containing a chitinase A (GH18 family) N-terminal domain (ERIC2_c09520) was identified in both genomes. The protein sequences deduced from these pseudogenes showed significant protein sequence similarity with putative $C$. botulinum chitodextrinase [52]. However, although all Paenibacillus species genomes sequenced so far contain several chitinase genes, no entire and putatively functional chitinase gene could be detected in the genomes of $P$. larvae DSM 25719 and DSM 25430 posing the intriguing question how the described chitin-degradation by $P$. larvae during infection [9] is achieved.

Enhancin-like protease. In the genome of DSM 25719 and DSM 25430 genes encoding a metalloendopeptidase of the enhancin family were present. Enhancin was originally described for granuloviruses $(\mathrm{GVs})$ and plays an important role in viral infection. Enhancin is incorporated into viral occlusion bodies, which are ingested by the host. The occlusion bodies are broken down in the midgut of the host and the enhancin is released. Subsequently, enhancin disrupts the protective peritrophic matrix (PM), allowing the virion to enter the epithelial cells of the insect gut. The PM has a lattice structure formed by chitin and insect intestinal mucin (IIM), and the viral enhancin protein targets the IIM for degradation [53,54]. A similar mode of action has been described for the bacterial enhancin-like protein of Bacillus thuringiensis (Bel) that exhibits 20 to $30 \%$ amino acid identity to viral enhancin proteins and $95 \%$ identity to enhancin-like proteins from other bacteria such as Yersinia pestis, B. anthracis, and B. cereus [55]. Thus, enhancin-like proteases from $P$. larvae might enhance bacterial infection by degradation of the peritrophic matrix (PM) of the insect midgut. However, the orthologous genes in $P$. larvae DSM 25719 (ERIC1_1c37500/ERIC1_1c37520) and P. larvae DSM 25430 (ERIC2_c09380-ERIC2_c09400) are dysfunctional due to insertion of transposases or frameshift mutations.

Serine proteases. Serine proteases are ubiquitous enzymes with a nucleophilic Ser residue at the active site and believed to constitute nearly one-third of all the known proteolytic enzymes. They function in diverse biological processes such as digestion, blood clotting, fertilization, development, complement activation, pathogenesis, apoptosis, immune response, secondary metabolism, with imbalances causing diseases like arthritis and tumors [56,57]. The genomes of $P$. larvae DSM 25719 and P. larvae DSM 25430 contain 11 and 6 genes coding for family S8 peptidases, respectively (Table $\mathrm{S} 1$, Table $\mathrm{S} 2$ ). Beside the additional proteases of DSM 25719 some of the remaining have no ortholog within the DSM 25430 genome. For instance $P$. larvae DSM 25719 gene ERIC1_1c21520 no ortholog could be found in the sequenced $P$. larvae DSM 25430 genome and ortholog ERIC1_1c05410 gene is interrupted into two pseudogenes (ERIC2_c16890ERIC2_c16910) by a transposase in the sequenced DSM 25430 genome (Figure 4A). All tested $P$. larvae ERIC I-strains harbored these two serine protease genes. The serine protease of DSM 25430 is probably non-functional due to an inserted transposase. To test whether or not all strains of $P$. larvae ERIC II indeed lack a functional serine protease, we screened a collection of ERIC II strains for the inserted transposase: one out of ten tested strains did not give any signal for this gene while seven of the tested strains carried the inserted transposase and another two still harbored the non-disrupted serine protease gene (Figure 4B). Therefore, the observed inactivation of this serine protease gene is strain-specific within the P. larvae genotype ERIC II.

Proteases for escaping immune response. Infected larvae activate an immune response already early during infection by upregulating expression of toll receptors, antimicrobial peptides and lysozyme $[55,58,59]$. However, rescue of infected larvae by immune response has never been observed, suggesting that $P$. larvae can counteract the larval immune defense quite efficiently. 
A
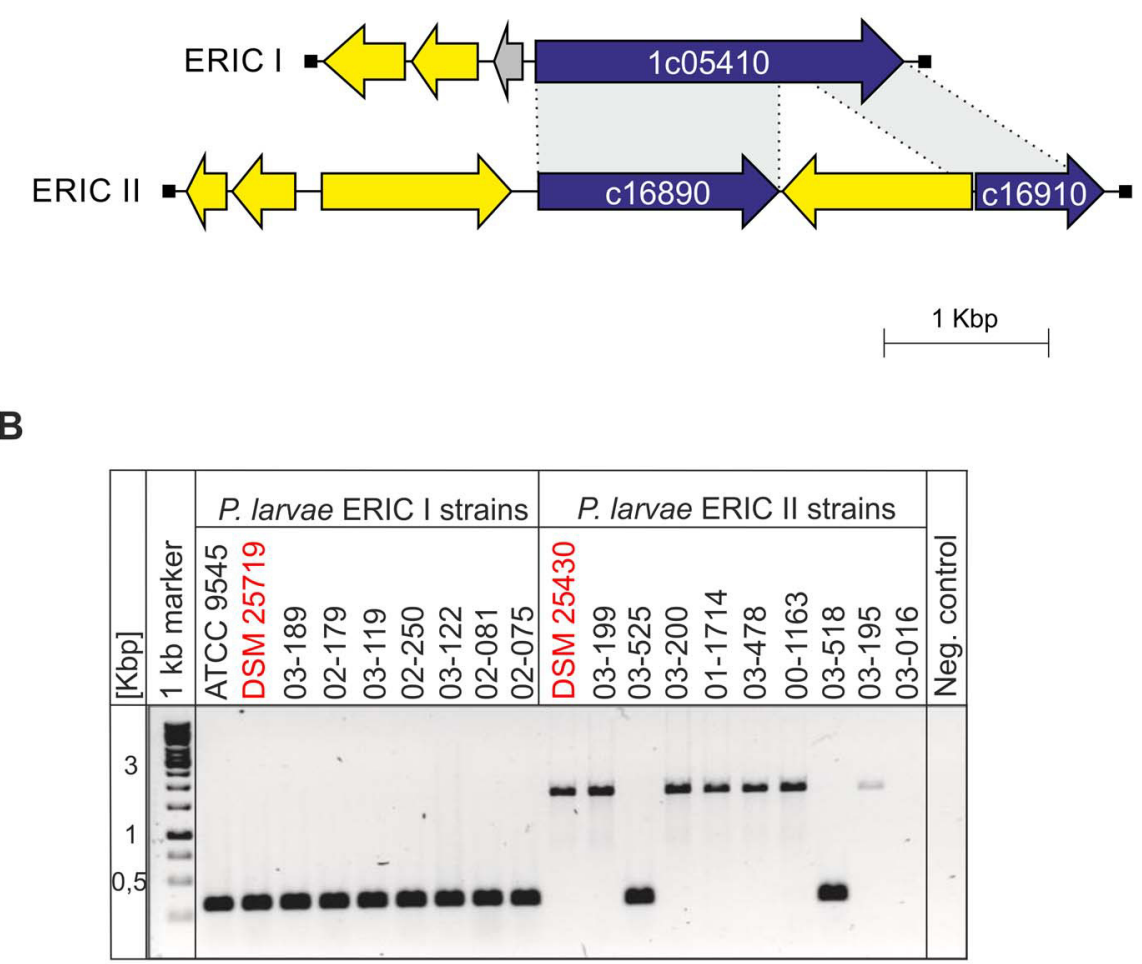

Figure 4. Genetic organization of subtilisin-like serine protease gene locus. Related ORFs are shown in the same following colors: blue, subtilisin-like serine protease gene; yellow, transposases/integrases; grey, genomic context (A). The similarities between pairs of sequences are depicted. PCR screening of ERIC I and ERIC II strains for functional or disrupted subtilisin-like serine protease genes (B). doi:10.1371/journal.pone.0090914.g004

We identified virulence-associated genes, which might be involved in this function. In P. larvae DSM 25430, the gene ERIC2_c27330 encodes a metalloprotease that belongs to the M6 peptidase family. Interestingly, this protein shows significant amino acid identity $(41 \%)$ to the immune inhibitor A precursor (InhA), a virulence factor encoded by the inhA gene of B. thuringiensis. InhA of $B$. thuringiensis specifically cleaves antibacterial peptides produced by insect hosts [60]. We hypothesize that the putative $P$. larvae InhA has a similar function and helps $P$. larvae to survive the larval immune response. However, this gene (ERIC1_1c15040) is interrupted by a frameshift mutation in all $P$. larvae ERIC I strains analyzed so far, suggesting that it is non-functional in ERIC I genotype.

\section{Secondary Metabolites}

Microorganisms are often capable of producing metabolites, which have a secondary role in self-defense or aggression [61]. Polyketide synthases (PKS) and nonribosomal peptide synthetases (NRPS) are the producers of two large groups of natural products with remarkable structural diversity and biological activities, including antibiotic, antifungal, anticancer, immunosuppressant and cholesterol-lowering activities [62]. We recently presented evidence for the existence of NRPS-PKS clusters in the genomes of all P. larvae genotypes [28]. In the sequenced genomes, we identified four different NRPS or PKS clusters (Figure 5). Both genomes seem to encode two PKS-NRPS hybrid clusters and two NRPS clusters. Three of these clusters could be assigned with a putative function; the biological role of the other cluster remains unknown.
The first NRPS/PKS cluster with a predicted function (strain DSM 25430, Figure 5A) is putatively encoding an iturin family lipopeptide antibiotic. Iturin family antibiotics are heptapeptides with a $\beta$-amino fatty acid that show strong antifungal activity $[63,64]$. For example, iturin A destroys the fungal cytoplasmic membrane, which leads to transmembrane channels, permitting the release of vital ions such as $\mathrm{K}^{+}$from the fungal cells. While the antibacterial activities of the iturin family antibiotics are limited [65], a lytic activity against human erythrocytes could be detected [66]. ORF arrangement and the domain organizations showed a high similarity to mycosubtilin, iturin $\mathrm{A}$ and bacillomycin synthetase [67,68]. The putative DSM 25430 operon spanned $37 \mathrm{~kb}$. We suggest that this putative iturin family synthetase mainly exhibits antifungal and antibacterial activity, but lytic activity against hemocytes in the hemolymph of the honey bee larvae is also conceivable [69]. However, the corresponding DSM 25719 region was significantly shorter $(12.5 \mathrm{~kb})$.

The second NRPS cluster found in both genomes showed similarity to the gramicidin S synthetase (Figure 5B). Gramicidin S is a potent cyclopeptide antibiotic, as it interacts with the cell membrane of target microorganisms and disrupts it [70]. The third large NRPS/PKS-cluster was also present in both strains (Figure 5C). It comprised approximately $60 \mathrm{~kb}$ and exhibited no similarity to known NRPS/PKS clusters.

Expression of the fourth NRPS cluster will putatively result in the production of a siderophore with similarity to bacillibactin. Siderophores are low-molecular mass microbial compounds with a very high affinity for iron [71], especially $\mathrm{Fe}^{3+}$ [72]. The genes involved in the biosynthetic pathway for bacillibactin in B. subtilis have been characterized [73] and compared with paenibactin, a 
A

c18780

c18770

c18760

c18750
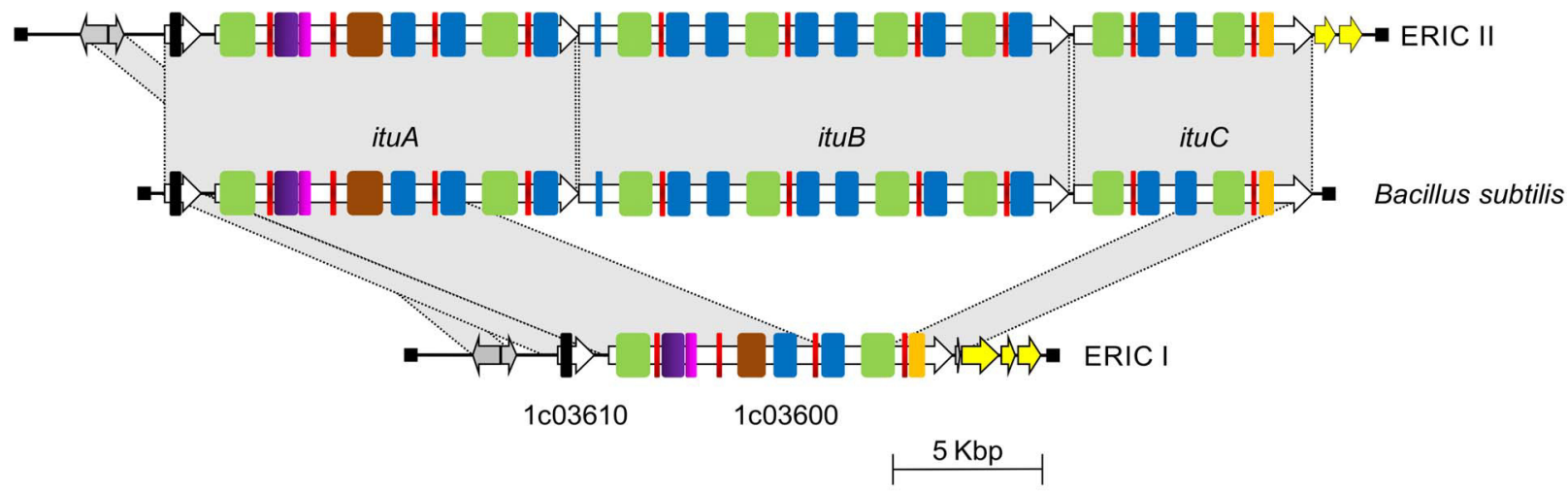

B

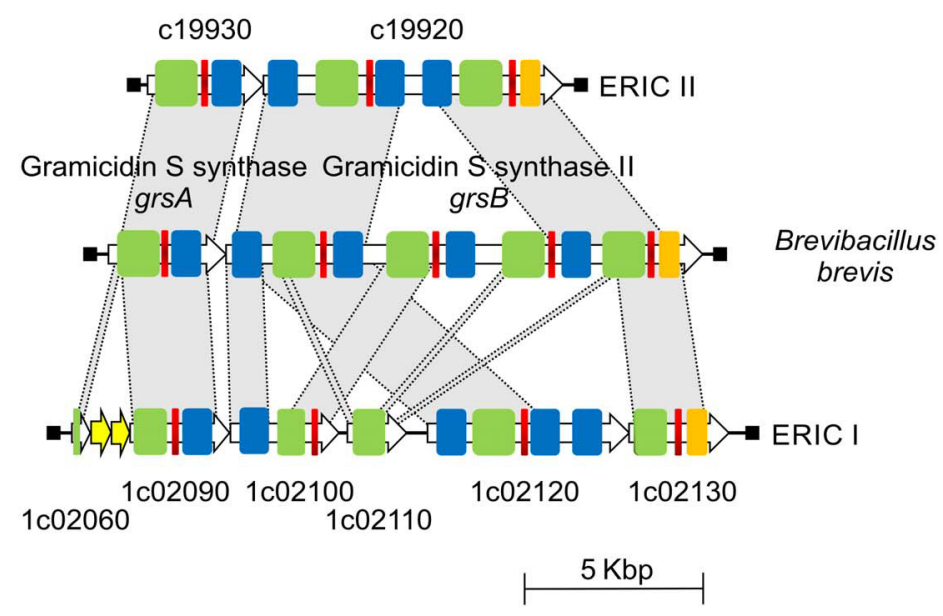

Thioesterase domain AMP-binding domain

Phosphopantetheine attachment site (PP-binging domain) Condensation domain

Beta-ketoacyl synthase, Nterminal and $\mathrm{C}$-terminal domain

Acyltransferase domain

KR domain

Aminotransferase class-III domain

Methyltransferase domain

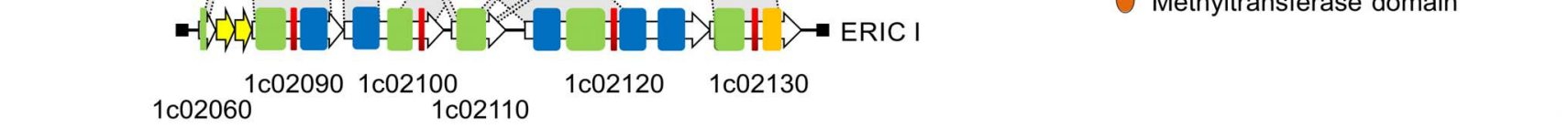

C

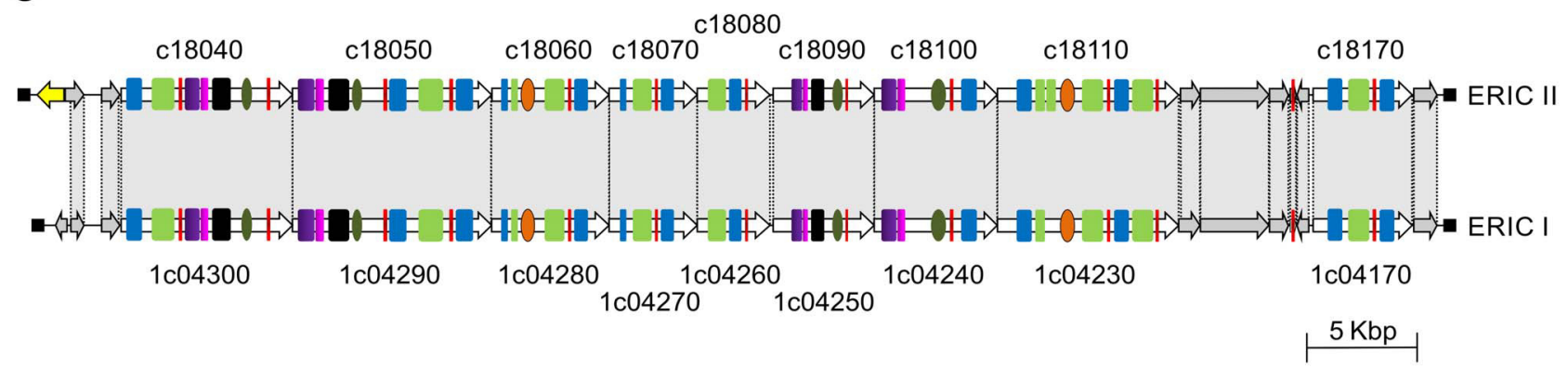

D

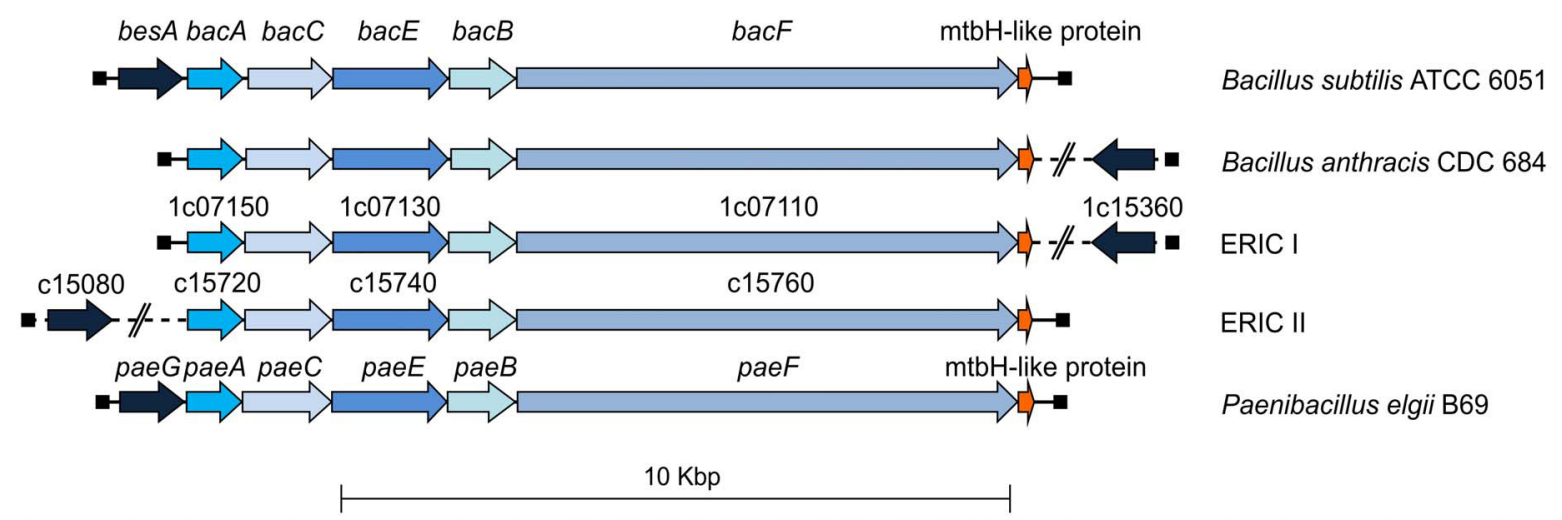

Figure 5. Genetic organization of $P$. larvae PKS/NRPS biosynthesis gene clusters. Shown are the PKS/NRPS biosynthesis gene clusters from P. larvae DSM 25719 (ERIC I) and DSM 25430 (ERIC II) genes. Iturin family lipopeptide antibiotic NRPS/PKS cluster (A), gramicidin S synthase NRPS 
cluster (B), organization and architecture of a novel NRPS/PKS cluster in ERIC I and ERIC II (C), and the bacillibactin/paenibactin NRPS cluster (D). Related ORFs are shown in the same colors.

doi:10.1371/journal.pone.0090914.g005

catecholate siderophore produced by Paenibacillus elgii B69 [74]. The paenibactin gene cluster consisted of six genes (paeGACEBF), of which three contained NRPS-domains (paeE, paeB and paeF). The genomes of DSM 25719 encoded gene clusters, which showed high similarity to the paenibactin gene cluster of $P$. elgii B69 (Figure 5D).

In the genomes of DSM 25719 and DSM 25430, we also identified ORFs coding for proteins with high similarity to a lanthionine synthetase, suggesting that $P$. larvae produces lantibiotics. Lantibiotics are a unique class of peptide antibiotics. Lantibiotics are small antimicrobial agents (19-38 amino acids) derived from ribosomally synthesized peptides. They are produced by Firmicutes, and include mutacin, subtilin, and nisin. Many lantibiotics are bacteriocidal against a variety of Gram positive bacteria at nanomolar levels [75]. In the genome of DSM 25719, we found three lantibiotic biosynthesis clusters (Figure 6). Due to this genomic difference between DSM 25719 and DSM 25430, suppression subtractive hybridization analysis was successful in already predicting the potential for the production of lantibiotics at least for DSM 25719 [28].

\section{Clustered Regulatory Interspaced Short Palindromic Repeats (CRISPRs)}

CRISPRs systems are genomic systems for host defense against invasive nucleotide sequences such as phages and plasmids. CRISPRs consist of a genomic repeat region, the CRISPR in sensu stricto and associated proteins the CRISPR-associated proteins (CAS; CRISPR/Cas) or Cas module-RAMP (RepeatAssociated Mysterious Proteins) systems (CRISPR/Cmr). A CRISPR locus is a class of direct repeats, which contain unique, target specific spacer sequences of similar length located between each pair of repeats [76,77]. Genome analysis showed the presence of seven possible CRISPRs candidates in ERIC I whereas in ERIC II only one candidate was found [78] (Table S4, Table S5).

In the genome of DSM 25719 CRISPR/Cas cluster was found (ERIC1_2c05680-ERIC1_2c05750) and comparative analysis showed that it was DSM 25719-specific. Downstream of ERIC1_2c05750 gene we identified five direct repeats and the transposase. Additionally, a CRISPR/Cmr cluster was found in both sequenced strains (DSM 25719: ERIC1_1c30330ERIC1_1c30350; and DSM 25430: ERIC2_c04120ERIC2_c04180). However, in strain DSM 25719 genes $c m r 5$ and cmr6 are missing. In both strains this cluster is flanked by transposases. It has been suggested that CRISPRs play a role in chromosomal rearrangement [79] and appear to be among the most rapidly evolving elements in the genome. Closely related species and strains differ in their CRISPR composition [80,81].

\section{Pathogenicity Islands (PAIs)}

To identify orthologous genes, as well as strain/genotypespecific gene content a bidirectional BLAST was employed. DSM 25719 and DSM 25430 genomes share a large core genome. Island-like genomic regions (GI) encoding a variety of putative virulence-associated and fitness-associated traits were identified. The DSM 25719 genome contained 23 regions larger than $5 \mathrm{~kb}$, excluding predicted prophage regions (Figure 2, Table S6). Most of the genes within the DSM 25719-specific regions encoded proteins of unknown function or hypothetical proteins. Nevertheless, a significant number of mobile elements such as insertion elements, transposases, integrases, and recombinases were identified within each region. The above-described toxin loci in general belong to the P. larvae ERIC I-specific gene pool.
A
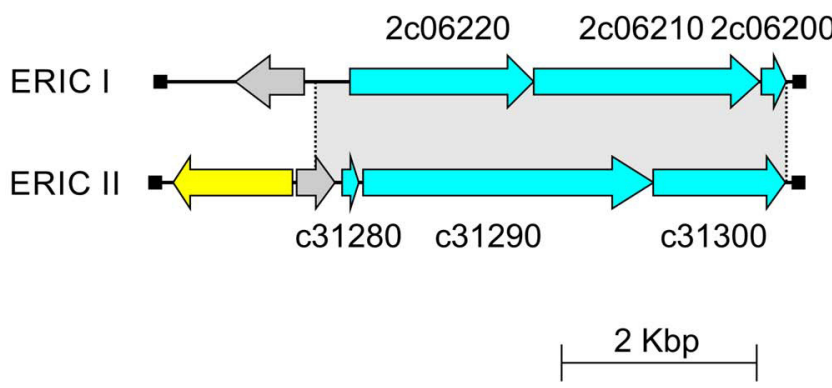

B
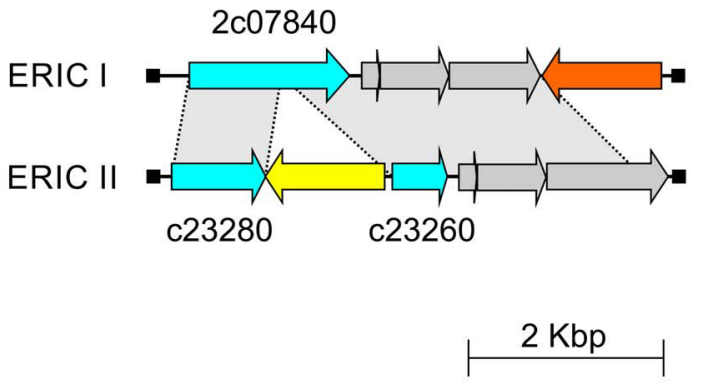

C

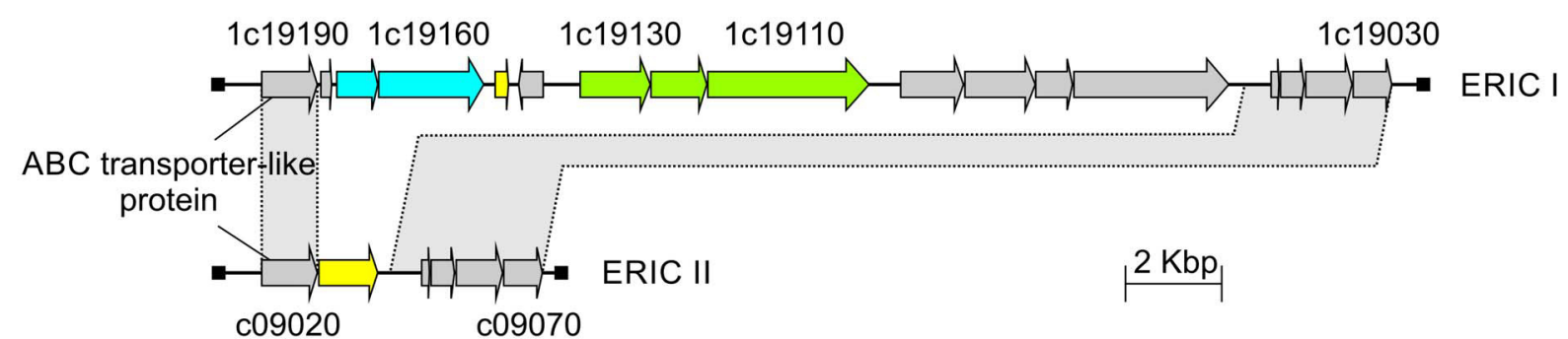

Figure 6. Genetic organization of lantibiotic biosynthesis clusters from P. larvae DSM 25719 (ERIC I) and DSM 25430 (ERIC II). Related ORFs are shown in the same following colors: yellow, transposases; grey, genome context; green, type I restriction system; cyan, lantibiotic biosynthesis clusters; and orange, integrase. The arrows indicate the direction of transcription.

doi:10.1371/journal.pone.0090914.g006 
The largest DSM 25719-specific region (GI21) spanned over $120 \mathrm{~kb}$ and comprised the genes ERIC1_2c04540 (integrase family protein) to ERIC1_2c05970. This region encoded a cas operon, subtilisin E, ferrous iron transport proteins, amino acid permease, putative transporter proteins, and toxin locus Tx7. GI5 (ORFs: ERIC1_1c03660-ERIC1_1c04000) encoded 35 predicted proteins, including putative O-methyltransferase, type-2 restriction enzyme BsuBI and iron transport system isd. GI19 represents the DNA region $(21 \mathrm{~kb})$ ranging from ERIC1_2c02250 to ERIC1_2c02370. It harbored 13 predicted proteins with similarities to RHS repeat-associated core domain-containing protein, secreted proteins, and cell surface proteins. GI10 (ERIC1_1c19060-ERIC1_1c19180) encoded 13 ORFs, including SMC domain protein, type I restriction-modification system and a lantibiotic-modifying enzyme. These ORFs were absent in the DSM 25430 genome. Above described toxin loci could also be identified within DSM 25719-specific genomic regions such as toxin locus Plx1 within GI1, and toxin loci Plx5, Plx3, Tx6 within GI2, GI8, and GI13, respectively.

In contrast to DSM 25719 the DSM 25430 genome contained only three $P$. larvae ERIC II-specific regions that exceeded $5 \mathrm{~kb}$, excluding putative phage regions (Table S7). The largest DSM 25430-specific region (GI2) spanned over $25 \mathrm{~kb}$, comprised the genes ERIC2_c18730 to ERIC2_c18760, and harbored genes with similarity to iturin A biosynthesis cluster. Only part of the cluster could be found within the DSM 25719 genome. The two other genomic regions encoded insertion elements and hypothetical proteins but also enzymes like amidinotransferase, alpha/beta hydrolase, monogalactosyldiacylglycerol synthase (GI1) or bacitracin export ATP-binding protein (GI3).

\section{Conclusions}

It has been shown that the bacterial life cycle in infected larvae can be divided into two stages (Figure 7) [8]. The genome analysis identified genes, which may encode for all crucial steps of the known life cycle. The early phase of infection is non-invasive and includes ingestion of spore-contaminated food, and subsequently spore germination and proliferation in the midgut lumen. The vegetative bacteria proliferate massively in the midgut lumen prior to breaching the epithelium. During this non-invasive stage $P$. larvae can be considered a commensal bacterium living from the content of the larval diet, i.e., sugars like glucose and fructose. Indeed, $P$. larvae is able to metabolize different sugars and sugar derivatives [12,82] through several metabolic pathways identified in this study. Although $P$. larvae does not actively attack the infected larvae at this stage of infection, the bacteria are living on the expense of the larvae by competing for incoming food. Therefore, it is not surprising that a recent study of infected larvae using comparative proteomics revealed that infected larvae express higher levels of mitochondrial metabolic enzymes and deplete their energy stores during infection [55]. Thus an enhanced energy demand of infected larvae compared to non-infected larvae is indicated.

The non-invasive phase is followed by penetration of the midgut epithelium and subsequent invasion of the haemocoel via the paracellular route by sequentially destroying the peritrophic matrix, cell-cell junctions, the extracellular matrix, and the larval remains [8]. Bacterial factors likely to be involved in this process are different toxins and secreted extracellular proteases. The extraordinary proteolytic capacity of $P$. larvae has been the topic of many studies [16,41,83,84]. In both genotypes, our DNA sequence-based gene prediction supports the existence of a high number of proteases, which might be involved in several mechanisms during the invasive stage. (i) Proteases able to degrade antimicrobial peptides might neutralize the local immune response mounted by the epithelial cells and help $P$. larvae to survive in the midgut lumen and attack the epithelial barrier. (ii) Once the bacteria have access to the epithelium, proteases, possibly in conjunction with toxins, might be responsible for the disruption of the epithelial barrier integrity by degrading cell-cell and cellmatrix junctions. (iii) Collagenases are then needed to degrade the basement membrane and facilitate access to the underlying tissues. (iv) Once the larvae are dead, proteases are further needed for the subsequent degradation of the larval remains (ropy stage). Pure cultures of $P$. larvae can be obtained from dead larvae at this stage of the pathogenic process [85], a fact that can now be explained by the expression of potent antibiotics as identified in the genomes of both $P$. larvae genotypes. Vegetative bacteria then undergo sporulation and the formed spores drive disease transmission within the colony when disseminated to and ingested by the next larvae.

We have answered many questions with respect to the molecular pathogenesis of $P$. larvae by identifying several novel and important virulence factors of $P$. larvae involved in different steps in pathogenesis. However, our data also raise a new question: It has been shown that $P$. larvae genotype ERIC II is more virulent on the larval level than genotype ERIC I. Surprisingly, we now discovered that the toxins identified in ERIC I (strain DSM 25719) are lacking in the genome of ERIC II (DSM 25430). DSM 25430 strain only harbors non-functional remnants of toxin orthologs found in DSM 25719. In addition, many ERIC II strains lack several proteases (enhancin-like protease, serine proteases), which might also be of central importance for pathogenesis. Considering the high number of predicted genes with unknown function in both genomes (2133 in DSM 25719 and 1400 in DSM 25430) it is possible that unknown toxins or some other novel virulence factor are encoded by these genes. Our genome analysis showed that, although both genotypes are lethal for infected larvae and degrade the larval remains to a ropy mass, they obviously developed different modes of attacking and killing honey bee larvae. Understanding these differences in pathogenesis and elucidating the different virulence mechanisms is a prerequisite for the development of specific treatments against both $P$. larvae genotypes.

\section{Materials and Methods}

\section{Bacterial Strains and Culture Conditions}

The strains used in this study are given in Table S3. Paenibacillus larvae strains DSM 25719 and DSM 25430 were streaked out on Columbia sheep blood agar plates and incubated at $37^{\circ} \mathrm{C}$ for 3 days. For starter cultures, $5 \mathrm{ml} 2 \times$ MYPGP broth were inoculated with a single colony and incubated for $10-12$ hours at $37^{\circ} \mathrm{C}$. For main cultures, bacterial cells from the starter culture were diluted sixtyfold in a total volume of $300 \mathrm{ml} 2 \times$ MYPGP, incubated at $37^{\circ} \mathrm{C}$ overnight with moderate shaking until reaching the exponential phase.

\section{Isolation of Bacterial DNA and Plasmids}

DNA isolation was performed using the MasterPure Gram Positive Purification Kit (Epicentre) following the manufacturer's instructions. Briefly, bacterial cells were harvested, treated with lysozyme, Gram positive cell lysis solution, proteinase $\mathrm{K}$, protein precipitation reagent, and RNase A. DNA was precipitated by isopropanol, washed with ethanol and suspended in $60 \mu \mathrm{l}$ elution buffer (10 mM Tris-Cl, pH 8.5). The absence or presence of the subtilisin-like serine protease gene and possible mutations were tested by PCR. Briefly, P. larvae genomic DNA isolated from 


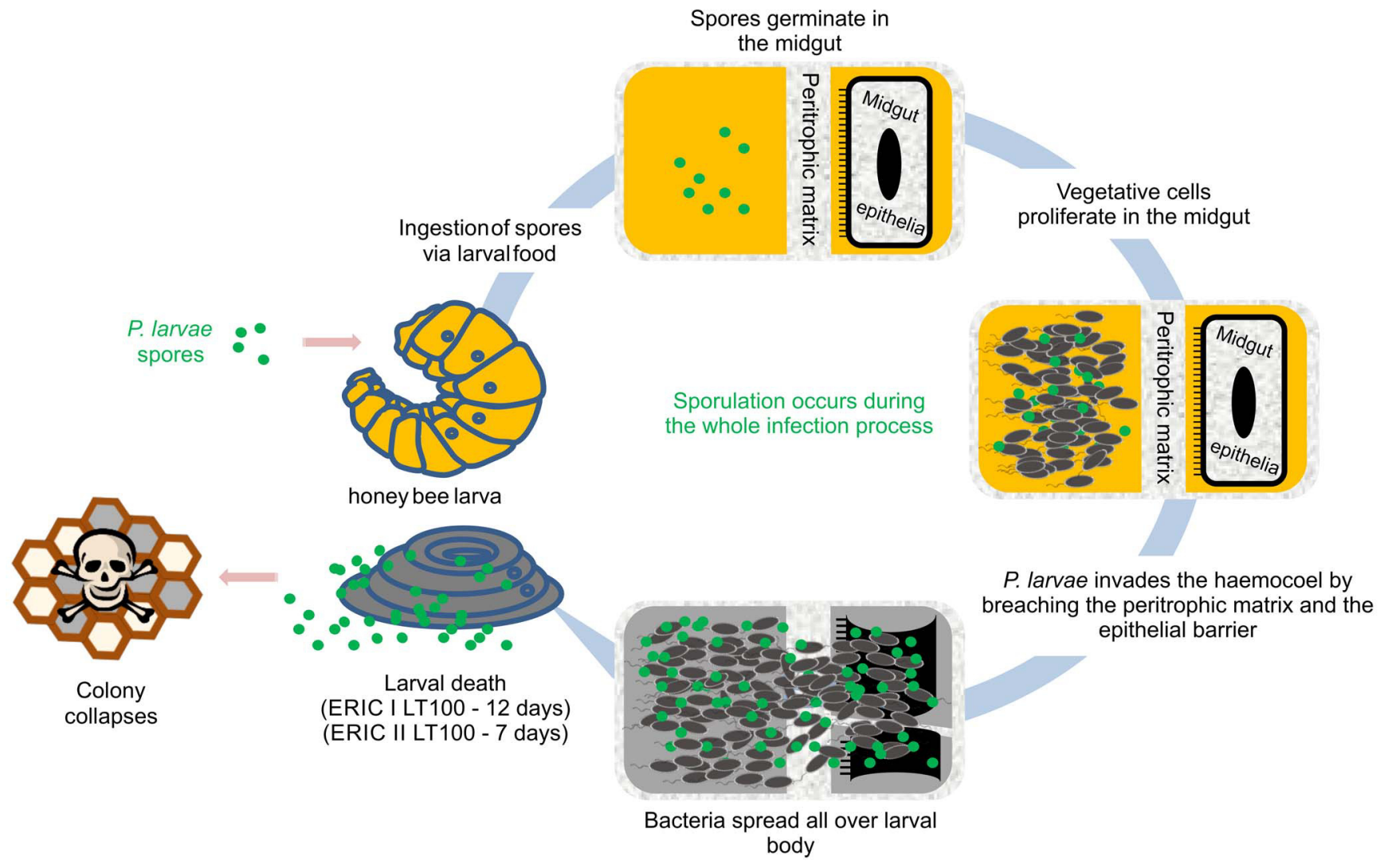

Figure 7. $P$. larvae infection model. doi:10.1371/journal.pone.0090914.g007

different strains (Table S3) was used as template for PCR amplification (primer pair ERC0390F, GATTCCAATTTGATCAACGA and ERC03906R, TCTGCACTGGAGTTAGTGTA) using a thermal cycler My Cycler ${ }^{\mathrm{TM}}$ apparatus (Bio-Rad, Munich, Germany) and a PCR kit (Qiagen, Hilden Germany). PCR amplification was performed with an initial heat activation of the HotStarTaq DNA Polymerase (Qiagen, Hilden, Germany) at $95^{\circ} \mathrm{C}$ for $5 \mathrm{~min}$, followed by 25 cycles of $30 \mathrm{sec}$ denaturation $\left(94^{\circ} \mathrm{C}\right), 1$ min annealing $\left(56^{\circ} \mathrm{C}\right)$ and 1 min elongation $\left(72^{\circ} \mathrm{C}\right)$, with a final elongation step of $10 \mathrm{~min}\left(72^{\circ} \mathrm{C}\right)$. Plasmid preparation was performed using the QIAprep Spin Miniprep kit (Qiagen, Hilden, Germany) as recommended by the manufacturer.

\section{Genome Sequencing, Assembly and Gap Closure}

The extracted DNA was used in a combined sequencing approach using a 454 GS-FLX Titanium XL system with Titanium chemistry (Roche Life Science, Mannheim, Germany) and the Genome Analyzer II system (Illumina, San Diego, CA, USA) as recommended by the manufacturers. Resulting reads were assembled into contigs using MIRA software. For $P$. larvae DSM 25719 (ERIC I), shotgun sequencing resulted in 38.35-fold and 56.49-fold coverage from 454 and Illumina reads, respectively. In case of $P$. larvae DSM 25430 (ERIC II), an average coverage of 64.4-fold was determined (30.07-fold 454 coverage and 35.01fold Illumina coverage). Editing of shotgun sequences and 454 sequences were done by using GAP4, as part of the Staden software package [86]. To solve problems with misassembled regions caused by repetitive sequences and close remaining sequence gaps, PCR reactions, combinatorial multiplex PCRs reactions, fosmid libraries, plasmid libraries, and primer walking with recombinant plasmids were used. PCR reactions have been carried out with the BioXact Kit (Qiagen, Hilden, Germany) and Phusion High Fidelity DNA Polymerase Kit (Thermo Fisher Scientific, Schwerte, Germany) as described by the manufacturers.

\section{Bioinformatic Tools}

Automatic gene prediction and functional annotation of the protein-coding genes were initially carried out with the IMG/ER (Intergrated Microbial Genomes/Expert Review) system [87]. Subsequently, gene prediction and annotation were manually curated by using the Swiss-Prot, TREMBL and InterPro databases. Complete genome comparisons were done using protein-based bidirectional BLAST. The genome sequences reported in this paper have been deposited in the GenBank database under accession numbers ADFW00000000 (DSM 25430) and CP003355-CP003356 (DSM 25719). Phage regions were predicted by employing PHAST (PHAge Search Tool) [22] and manually corrected. Visualization of plasmid comparisons was done with the ACT program from the Sanger Institute (http:// www.sanger.ac.uk/) [88].

\section{RNA Extraction and Transcript Analysis}

Total RNA was isolated either from bacteria grown in MYPGP at $37^{\circ} \mathrm{C}$ during the log-phase of growth or from experimentally infected larvae [5,7] at day 4 post infection using Qiagen RNeasy Mini Kit according to the manufacturer's protocol. An additional DNase step (DNase RNase-free, Qiagen) was included to remove bacterial (and larval) genomic DNA. Analysis of mRNA expression was performed using Omniscript RT (Qiagen) and cDNA was prepared from a total of $500 \mathrm{ng}$ of RNA using random hexamer 
primers. The derived cDNA was used as template in PCR reactions with specific primers.

\section{Supporting Information}

Figure S1 Analysis of $P$. larvae plasmids pPLA1_10 and pPLA2_10.

(PDF)

Table S1 Peptidases identified and classified in the genome of $P$. larvae strain DSM 25719.

(PDF)

Table S2 Peptidases identified and classified in the genome of $P$. larvae strain DSM 25430.

(PDF)

Table S3 P. larvae strains used in this study. (PDF)

Table S4 CRISPR analysis of the $P$. larvae strain DSM 25719 genome.

(PDF)

\section{References}

1. Morse RA, Calderon NW (2000) The value of honey bees as pollinators of U.S. Crops in 2000. Bee Culture 128: 1-15.

2. Aizen M, Garibaldi L, Cunningham S, Klein A (2008) Long-term global trends in crop yield and production reveal no current pollination shortage but increasing pollinator dependency. Curr Biol 18: 1572-1575.

3. Aizen MA, Harder LD (2009) The global stock of domesticated honey bees is growing slower than agricultural demand for pollination. Curr Biol 19: 915-918.

4. Genersch E (2010) Honey bee pathology: current threats to honey bees and beekeeping. Appl Microbiol Biotech 87: 87-97.

5. Genersch E, Forsgren E, Pentikäinen J, Ashiralieva A, Rauch S, et al. (2006) Reclassification of Paenibacillus larvae subsp. pulvifaciens and Paenibacillus larvae subsp. larvae as Paenibacillus larvae without subspecies differentiation. Int J Syst Evol Microbiol 56: 501-511.

6. Brodsgaard CJ, Ritter W, Hansen H (1998) Response of in vitro reared honey bee larvae to various doses of Paenibacillus larvae larvae spores. Apidologie 29: 569578.

7. Genersch E, Ashiralieva A, Fries I (2005) Strain- and genotype-specific differences in virulence of Paenibacillus larvae subsp. larvae, the causative agent of American foulbrood disease in honey bees. Appl Environ Microbiol 71: 75517555 .

8. Yue D, Nordhoff M, Wieler LH, Genersch E (2008) Fluorescence in situhybridization (FISH) analysis of the interactions between honeybee larvae and Paenibacillus larvae, the causative agent of American foulbrood of honeybees (Apis mellifera). Environ Microbiol 10: 1612-1620.

9. Garcia-Gonzalez E, Genersch E (2013) Honey bee larval peritrophic matrix degradation during infection with Paenibacillus larvae, the etiological agent of American Foulbrood of honey bees, is a key step in pathogenesis. Environ Microbiol 15, 2894-2901.

10. Genersch E, Otten C (2003) The use of repetitive element PCR fingerprinting (rep-PCR) for genetic subtyping of German field isolates of Paenibacillus larvae subsp. larvae. Apidologie 34: 195-206.

11. Forsgren E, Stevanovic J, Fries I (2008) Variability in germination and in temperature and storage resistance among Paenibacillus larvae genotypes. Vet Microbiol 129: 342-349.

12. Neuendorf S, Hedtke K, Tangen G, Genersch E (2004) Biochemical characterization of different genotypes of Paenibacillus larvae subsp. larvae, a honey bee bacterial pathogen. Microbiology 150: 2381-2390.

13. Rauch S, Ashiralieva A, Hedtke K, Genersch E (2009) Negative correlation between individual-insect-level virulence and colony-level virulence of Paenibacillus larvae, the etiological agent of American foulbrood of honeybees. Appl Environ Microbiol 75: 3344-3347.

14. Alippi AM, Reynaldi FJ, Lopez AC, De Giusti MR, Aguilar OM (2004) Molecular epidemiology of Paenibacillus larvae larvae and incidence of American foulbrood in Argentinean honeys from Buenos Aires province. J Apic Res 43: $135-143$.

15. Peters M, Kilwinski J, Beringhoff A, Reckling D, Genersch E (2006) American foulbrood of the honey bee: Occurrence and distribution of different genotypes of Paenibacillus larvae in the administrative district of Arnsberg (North RhineWestphalia). J Vet Med B 53: 100-104.

16. Antúnez K, Anido M, Schlapp G, Evans JD, Zunino P (2009) Characterization of secreted proteases of Paenibacillus larvae, potential virulence factors involved in honeybee larval infection. J Invertebr Pathol 102: 129-132.
Table S5 GRISPR analysis of the $P$. larvae strain DSM 25430 genome.

(PDF)

Table S6 Strain-specific regions identified in the genome of $P$. larvae strain DSM 25719.

(PDF)

Table 57 Strain-specific regions identified in the genome of $P$. larvae strain DSM 25430.

(PDF)

\section{Acknowledgments}

We thank the $\mathrm{G}_{2} \mathrm{~L}$ sequencing team for the support.

\section{Author Contributions}

Conceived and designed the experiments: EB EG RD. Performed the experiments: MD EB AF KG LP EGG. Analyzed the data: MD EB AF JV LP HL EGG EG. Wrote the paper: MD EB EG RD.

17. Loncaric I, Derakhshifar I, Oberlerchner JT, Köglberger H, Moosbeckhofer R (2009) Genetic diversity among isolates of Paenibacillus larvae from Austria. J Invertebr Pathol 100: 44 46.

18. Genersch E (2007) Paenibacillus larvae and American foulbrood in honeybees. Berl Münch Tierärztl Wschr 120: 26-33.

19. Genersch E (2010) American Foulbrood in honeybees and its causative agent, Paenibacillus larvae. J Invertebr Pathol 103: S10-S19.

20. Qin X, Evans JD, Aronstein KA, Murray KD, Weinstock GM (2006) Genome sequences of the honey bee pathogens Paenibacillus larvae and Ascosphaera apis. Insect Mol Biol 15: 715-718.

21. Chan QW, Cornman RS, Birol I, Liao NY, Chan SK, et al. (2011) Updated genome assembly and annotation of Paenibacillus larvae, the agent of American foulbrood disease of honey bees. BMC Genomics 12: 450.

22. Zhou Y, Liang Y, Lynch K, Dennis JJ, Wishart DS (2011) PHAST: a fast phage search tool. Nucleic Acids Res 39 (suppl 2): W347-W352.

23. Mahillon J, Leonard C, Chandler M (1999) IS elements as constituents of bacterial genomes. Res Microbiol 150: 675-687.

24. Siguier P, Filée J, Chandler M (2006) Insertion sequences in prokaryotic genomes. Curr Opin Microbiol 9: 526-531.

25. Brodschneider R, Crailsheim K (2010) Nutrition and health in honey bees. Apidologie 41: 278-294.

26. Graham JM, editor (1992) The hive and the honey bee. Hamilton, IL: Dadant \& Sons.

27. Woodring J, Boulden M, Das S, Gäde G (1993) Studies on blood sugar homeostasis in the honeybee (Apis mellifera, L.). J Insect Physiol 39: 89-97.

28. Fünfhaus A, Ashiralieva A, Borriss R, Genersch E (2009) Use of suppression subtractive hybridization to identify genetic differences between differentially virulent genotypes of Paenibacillus larvae, the etiological agent of American foulbrood of honeybees. Environ Microbiol Rep 1: 240-250.

29. Barth H, Aktories K, Popoff MR, Stiles BG (2004) Binary bacterial toxins: biochemistry, biology, and applications of common Clostridium and Bacillus proteins. Microbiol Mol Biol Rev 68: 373-402.

30. Poppinga L, Janesch B, Fünfhaus A, Sekot G, Garcia-Gonzalez E, et al. (2012) Identification and functional analysis of the S-layer protein SplA of Paenibacillus larvae, the causative agent of American foulbrood of honey bees. PLoS Pathog. 8: e1002716.

31. Fünfhaus A, Poppinga L, Genersch E (2013) Identification and characterization of two novel toxins expressed by the lethal honey bee pathogen Paenibacillus larvae, the causative agent of American foulbrood. Environ Microbiol doi: $10.1111 / 1462-2920.12229$

32. Schirmer J, Just I, Aktories K (2002) The ADP-ribosylating mosquitocidal toxin from Bacillus sphaericus - proteolytic activiation, enzyme activity, and cytotoxic effects. J Biol Chem 277: 11941-11948.

33. Watanabe M, Enomoto S, Takamura-Enya T, Nakano T, Koyama K, et al. (2004) Enzymatic properties of Pierisin-1 and its N-terminal domain, a guaninespecific ADP-ribosyltransferase from the cabbage butterfly. J Biochem 135: 471477.

34. Lúquez C, Raphael BH, Maslanka SE (2009) Neurotoxin gene clusters in Clostridium botulinum type Ab strains. Appl Environ Microbiol 75: 6094-6101.

35. Nakano H, Okabe T, Hashimoto H, Sakaguchi G (1990) Incidence of Clostridium botulinum in honey of various origins. Jpn J Med Sci Biol 43: 183-195.

36. Snowdon JA, Gliver DO (1996) Microorganisms in honey. Int J Food Microbiol 31: $1-26$. 
37. de Maagd RA, Bravo A, Crickmore N (2001) How Bacillus thuringiensis has evolved specific toxins to colonize the insect world. Trends Genet 17: 193-9.

38. Petit L, Maier E, Gibert M, Popoff MR, Benz R (2001) Clostridium perfringens epsilon toxin induces a rapid change of cell membrane permeability to ions and forms channels in artificial lipid bilayers. J Biol Chem 276: 15736-15740.

39. Baida GE, Kuzmin NP (1995) Cloning and primary structure of a new hemolysin gene from Bacillus cereus. Biochim Biophys Acta 1264: 151-154.

40. Jarosz J, Glinski Z (1990) Selective inhibition of cecropin-like activity of insect immune blood by protease from American foulbrood scales. J Invertebr Pathol 56: $143-149$.

41. Dancer BN, Chantawannakul P (1997) The proteases of American foulbrood scales. J Invertebr Pathol 70: 79-87.

42. Rawlings ND, Morton FR (2008) The MEROPS batch Blast: a tool to detect peptidases and their non-peptidase homologues in a genome. Biochimie 90: 243-259.

43. Alberts B, Johnson A, Lewis J, Raff M, Roberts K, et al. (2002) The extracellular matrix of animals. Molecular Biology of the Cell. 4th edition. New York: Garland Science.

44. Jung C, Matsushita O, Katayama S, Minami J, Sakurai J, et al. (1999) Identification of metal ligands in the Clostridium histolyticum $\mathrm{ColH}$ collagenase. J Bacteriol 181, 2816-2822.

45. Keil B (1992) Vibrio alginolyticus ("Achromobacter") collagenase: biosynthesis, function, and application. Matrix Suppl 1: 127-133.

46. Matsushita O, Koide T, Kobayashi R, Nagata K, Okabe A (2001) Substrate recognition by the collagen-binding domain of Clostridium histolyticum class I collagenase. J Biol Chem 276: 8761-8770.

47. Féthière J, Eggimann B, Cygler M (1999) Crystal structure of chondroitin AC lyase, a representative of a family of glycosaminoglycan degrading enzymes. J Mol Biol 288: 635-647.

48. Stern R, Jedrzejas MJ (2006) Hyaluronidases: Their genomics, structures, and mechanisms of action. Chem Rev 106: 818-839.

49. Li SL, Kelly SJ, Lamani E, Ferraroni M, Jedrzejas MJ (2000) Structural basis of hyaluronan degradation by Streptococcus pneumonia hyaluronate lyase. EMBOJ 19: 1228-1240.

50. Lindsay AM, Zhang M, Mitchell Z, Holden MT, Waller AS, et al. (2009) The Streptococcus equi prophage-encoded protein SEQ2045 is a hyaluronan-specific hyaluronate lyase that is produced during equine infection. Microbiology 155 : 443-449.

51. Hegedus D, Erlandson M, Gillott C, Toprak U (2009) New insights into peritrophic matrix synthesis, architecture, and function. Annu Rev Entomol 54, 285-302.

52. Sebaihia M, Peck MW, Minton NP, Thomson NR, Holden MT, et al. (2007) Genome sequence of a proteolytic (Group I) Clostridium botulinum strain Hall A and comparative analysis of the clostridial genomes. Genome Res 17: 10821092.

53. Yamamoto T, Tanada Y (1978) Phospholipid, an enhancing component in the synergistic factor of a granulosis virus of the armyworm, Pseudaletia unipuncta. J Invertebr Pathol 31: 48-56.

54. Zhu Y, Hukuhara T, Tamura K (1989) Location of a synergistic factor in the capsule of a granulosis virus of the armyworm, Pseudaletia unipuncta. J Invertebr Pathol 54: 49-56.

55. Chan QWT, Melathopoulos AP, Pernal SF, Foster LJ (2009) The innate immune and systemic response in honey bees to a bacterial pathogen, Paenibacillus larvae. BMC Genomics 10: 387.

56. Hedstrom L (2002) Serine protease mechanism and specificity. Chem Rev 102: 4501-4524.

57. Barrett AJ (2004) Bioinformatics of proteases in the MEROPS database. Curr Opin Drug Discov Devel 7: 334-341.

58. Evans JD (2004) Transcriptional immune response by honey bee larvae during invasion by the bacterial pathogen, Paenibacillus larvae. J Invertebr Pathol 85. $105-111$.

59. Evans JD, Aronstein K, Chen YP, Hetru C, Imler JL, et al. (2006) Immune pathways and defence mechanisms in honey bees Apis mellifera. Insect Mol Biol 15: $645-656$.

60. Lövgren A, Zhang M, Engström A, Dalhammar G, Landén R (1990) Molecular characterization of immune inhibitor $\mathrm{A}$, a secreted virulence protease from Bacillus thuringiensis. Mol Microbiol 4: 2137-2146.

61. Khosla C, Gokhale RS, Jacobsen JR, Cane DE (1999) Tolerance and specificity of polyketide synthases. Annu Rev Biochem 68: 219-253.

62. Du L, Lou L (2010) PKS and NRPS release mechanisms. Nat Prod Rep 27: 255-278.
63. Thimon L, Peypoux F, Wallach J, Michel G (1995) Effect of the lipopeptide antibiotic, iturin $\mathrm{A}$, on morphology and membrane ultrastructure of yeast cells. FEMS Microbiol Lett 128: 101-106.

64. Arrebola E, Jacobs R, Korsten L (2010) Iturin A is the principal inhibitor in the biocontrol activity of Bacillus amyloliquefaciens PPCB004 against postharvest fungal pathogens. J Appl Microbiol 108: 386-395.

65. Maget-Dana R, Peypoux F (1994) Iturins, a special-classclass of pore-forming lipopeptides - biological and physicochemical properties. Toxicology 87: 151174.

66. Quentin MJ, Besson F, Peypoux F, Michel G (1982) Action of peptidolipidic antibiotics of the iturin group on erythrocytes - effect of some lipids on hemolysis. Biochim Biophys Acta 684: 207-211.

67. Duitman EH, Hamoen LW, Rembold M, Venema G, Seitz H, et al. (1999) The mycosubtilin synthetase of Bacillus subtilis ATCC 6633: A multifunctional hybrid between a peptide synthetase, an amino transferase, and a fatty acid synthase. Proc Natl Acad Sci U S A: 13294-13299.

68. Ansari MZ, Yadav G, Gokhale RS, Mohanty D (2004) NRPS-PKS: a knowledge-based resource for analysis of NRPS/PKS megasynthases. Nucleic Acids Res 32: W405-W413.

69. Gilliam M, Shimanuki H (1970) Total hemocyte counts of honey bee larvae (Apis mellifera L) from various elevations. Experientia 26: 1006-1007.

70. Tamaki M, Ishii R, Kikuchi S, Watanabe E (2005) A novel active analogue of gramicidin S with smaller ring size. J Antibiot (Tokyo) 58: 293-295.

71. Winkelmann G (2002) Microbial siderophore-mediated transport. Biochem Soc Trans 30: 691-696.

72. Raymond KN, Carrano CJ (1979) Coordination chemistry and microbial iron transport. Acc Chem Res 12: 183-190.

73. May JJ, Wendrich TM, Marahiel MA (2001) The $d h b$ operon of Bacillus subtilis encodes the biosynthetic template for the catecholic siderophore 2,3dihydroxybenzoate-glycine-threonine trimeric ester bacillibactin. J Biol Chem 276: 7209-7217.

74. Wen Y, Wu X, Teng Y, Qian C, Zhan Z, et al. (2011) Identification and analysis of the gene cluster involved in biosynthesis of paenibactin, a catecholate siderophore produced by Paenibacillus elgii B69. Environ Microbiol 13: 27262737.

75. Willey JM, van der Donk WA (2007) Lantibiotics: peptides of diverse structure and function. Annu Rev Microbiol 61: 477-501.

76. Bhaya D, Davison M, Barrangou R (2011) CRISPR-Cas systems in bacteria and archaea: versatile small RNAs for adaptive defense and regulation. Annu Rev Genet 45: 273-97.

77. Haft DH, Selengut J, Mongodin EF, Nelson KE (2005) A guild of 45 CRISPRassociated (Cas) protein families and multiple CRISPR/Cas subtypes exist in prokaryotic genomes. PLoS Comput Biol 1: e60.

78. Grissa I, Vergnaud G, Pourcel C (2007) CRISPRFinder: a web tool to identify clustered regularly interspaced short palindromic repeats. Nucleic Acids Res 35 (Web Server issue): W52-7.

79. Shah SA, Garrett RA (2011) CRISPR/Cas and Cmr modules, mobility and evolution of adaptive immune systems. Res Microbiol 162: 27-38.

80. Bolotin A, Quinquis B, Renault P, Sorokin A, Ehrlich SD, et al. (2004) Complete sequence and comparative genome analysis of the dairy bacterium Streptococcus thermophilus. Nat Biotechnol 22: 1554-1558.

81. Pourcel G, Salvignol G, Vergnaud G (2005) CRISPR elements in Tersinia pestis acquire new repeats by preferential uptake of bacteriophage DNA, and provide additional tools for evolutionary studies. Microbiology 151: 653-663.

82. Julian GS, Bulla LAJ (1971) Physiology of sporeforming bacteria associated with insects. J Bacteriol 108: 828-834.

83. Holst EC, Sturtevant AP (1940) Relation of proteolytic enzymes to phase of life cycle of Bacillus larvae and two new culture media for this organism. J Bacteriol 40: 723-731.

84. Hrabak J, Martinek K (2007) Screening of secreted proteases of Paenibacillus larvae by using substrate-SDS-polyacrylamide gel electrophoresis. J Apic Res 46: $160-164$.

85. Bailey L, Ball BV (1991) Honey bee pathology. London: Academic Press.

86. Staden R, Beal KF, Bonfield JK (2000) The Staden package, 1998. Methods Mol Biol 132: 115-130.

87. Markowitz VM, Mavromatis K, Ivanova NN, Chen IM, Chu K, et al. (2009) IMG ER: a system for microbial genome annotation expert review and curation. Bioinformatics $25: 2271-2278$.

88. Carver TJ, Rutherford KM, Berriman M, Rajandream MA, Barrell BG, et al. (2005) ACT: the Artemis Comparison tool. Bioinformatics 21: 3422-3423. 


\subsection{Ergänzende Daten zu Kapitel B2}

\section{Inhaltsverzeichnis}

Figure S1. Analysis of P. larvae plasmids pPLA1_10 and pPLA2_10.

Table S1. Peptidases identified and classified in the genome of P. larvae strain DSM 25719.

Table S2. Peptidases identified and classified in the genome of P. larvae strain DSM 25430.

Table S3. $P$. larvae strains used in this study.

Table S4. CRISPR analysis of the P. larvae strain DSM 25719 genome.

Table S5. CRISPR analysis of the $P$. larvae strain DSM 25430 genome.

Table S6. Strain-specific regions identified in the genome of P. larvae strain DSM 25719.

Table S7. Strain-specific regions identified in the genome of P. larvae strain DSM 25430. 
A

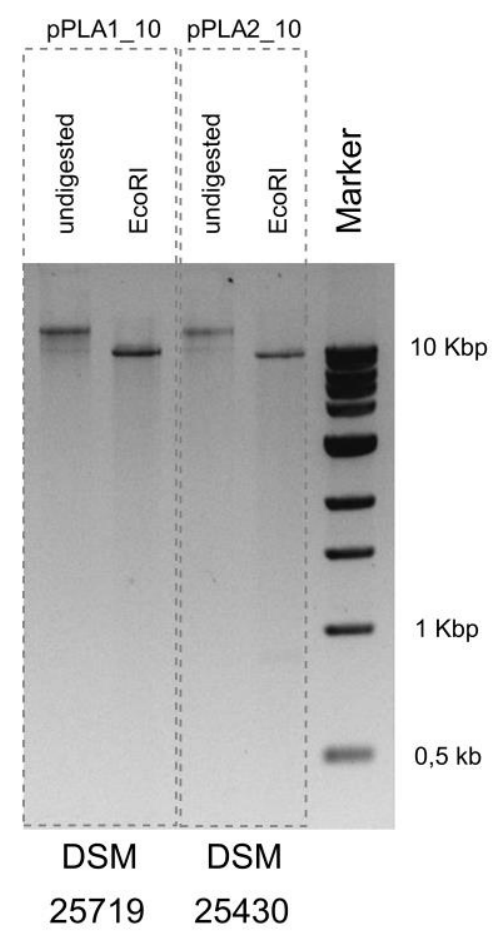

B

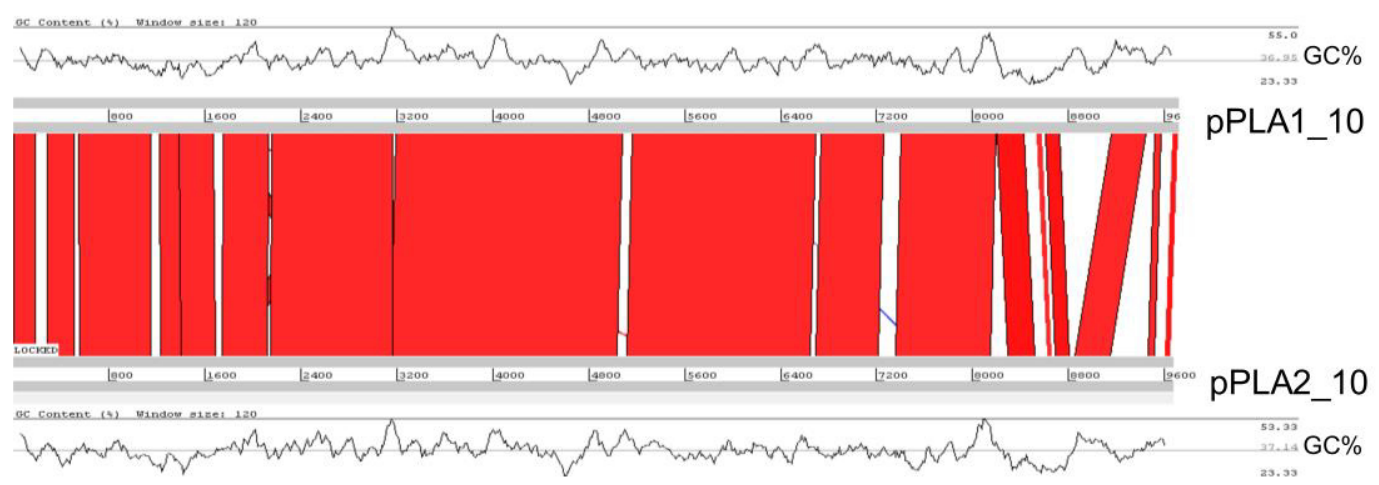

Figure S1: Analysis of P. larvae plasmids pPLA1_10 and pPLA2_10.

Restriction analysis of $P$. larvae plasmids pPLA1_10 and pPLA2_10 derived from P. larvae strains DSM 25719 and DSM 25430, respectively, (A) and pPLA1_10 and pPLA2_10 sequence comparisons (B). Comparative analysis was performed by employing the ACT software tool [1]. The relationships between each pair of sequences are depicted. Similar coding sequences were indicated by red lines.

\section{Reference}

1. Carver, T.J., Rutherford, K.M., Berriman, M., Rajandream, M.A., Barrel BG, et al. (2005). ACT: the artemis comparison tool. Bioinformatics 21: 3422-3423. 
Table S1. Peptidases identified and classified in the genome of $P$. larvae strain DSM 25719.

\begin{tabular}{|c|c|c|c|}
\hline Gene ID & MEROPS family [1] & MEROPS entry & E-value* \\
\hline ERIC1_1c10610 & A08 & MER070683 & $2.00 \mathrm{E}-12$ \\
\hline ERIC1_1c39320 & $\mathrm{A} 25$ & MER166302 & $7.70 \mathrm{E}-86$ \\
\hline ERIC1_1c10550 & $\mathrm{C} 26$ & MER146598 & $3.10 \mathrm{E}-89$ \\
\hline ERIC1_1c19730 & $\mathrm{C} 26$ & MER061258 & $1.40 \mathrm{E}-61$ \\
\hline ERIC1_1c23710 & $\mathrm{C} 26$ & MER134379 & $3.90 \mathrm{E}-66$ \\
\hline ERIC1_1c28490 & $\mathrm{C} 26$ & MER066434 & $1.90 \mathrm{E}-06$ \\
\hline ERIC1_1c18380 & $\mathrm{C} 40$ & MER003805 & $2.90 \mathrm{E}-28$ \\
\hline ERIC1_1c18810 & $\mathrm{C} 40$ & MER002450 & $4.50 \mathrm{E}-33$ \\
\hline ERIC1_1c20510 & $\mathrm{C} 40$ & MER003807 & $6.00 \mathrm{E}-18$ \\
\hline ERIC1_1c22900 & $\mathrm{C} 40$ & MER003805 & $1.90 \mathrm{E}-27$ \\
\hline ERIC1_1c25590 & $\mathrm{C} 40$ & MER003807 & $1.20 \mathrm{E}-20$ \\
\hline ERIC1_1c04050 & $\mathrm{C} 44$ & MER033254 & $1.40 \mathrm{E}-20$ \\
\hline ERIC1_1c13170 & $\mathrm{C} 44$ & MER033254 & $8.50 \mathrm{E}-20$ \\
\hline ERIC1_1c19450 & $\mathrm{C} 44$ & MER004101 & $7.50 \mathrm{E}-65$ \\
\hline ERIC1_1c19840 & $\mathrm{C} 44$ & MER020221 & $1.30 \mathrm{E}-28$ \\
\hline ERIC1_1c22530 & $\mathrm{C} 44$ & MER003327 & $1.60 \mathrm{E}-60$ \\
\hline ERIC1_1c36310 & $\mathrm{C} 44$ & MER033254 & $1.20 \mathrm{E}-16$ \\
\hline ERIC1_1c25870 & C56 & MER031432 & $2.70 \mathrm{E}-11$ \\
\hline ERIC1_4c00600 & C56 & MER002455 & $3.90 \mathrm{E}-32$ \\
\hline ERIC1_1c03910 & C60B & MER020433 & $1.30 \mathrm{E}-49$ \\
\hline ERIC1_1c12420 & $\mathrm{C} 82$ & MER076166 & $3.00 \mathrm{E}-08$ \\
\hline ERIC1_2c06260 & $\mathrm{C} 82$ & MER076166 & $5.70 \mathrm{E}-05$ \\
\hline ERIC1_2c05350 & S08A & MER055152 & $1.90 \mathrm{E}-65$ \\
\hline ERIC1_1c28020 & M01 & MER055808 & $1.70 \mathrm{E}-29$ \\
\hline ERIC1_1c16550 & M03B & MER001163 & $6.70 \mathrm{E}-68$ \\
\hline ERIC1_2c07220 & M03B & MER084913 & $8.50 \mathrm{E}-64$ \\
\hline ERIC1_1c04550 & M04 & MER001026 & $6.70 \mathrm{E}-104$ \\
\hline ERIC1_1c13440 & M04 & MER001026 & $3.90 \mathrm{E}-87$ \\
\hline ERIC1_1c39210 & M04 & MER001026 & $3.90 \mathrm{E}-87$ \\
\hline ERIC1_2c07760 & M04 & MER001026 & $6.80 \mathrm{E}-87$ \\
\hline ERIC1_1c24570 & M09B & MER001417 & $2.20 \mathrm{E}-119$ \\
\hline ERIC1_1c16780 & $\mathrm{M} 14 \mathrm{C}$ & MER001505 & $1.90 \mathrm{E}-78$ \\
\hline ERIC1_1c31420 & M15B & MER084164 & $5.90 \mathrm{E}-50$ \\
\hline ERIC1_1c00860 & M16B & MER142621 & $6.10 \mathrm{E}-90$ \\
\hline ERIC1_1c00860 & M16B & MER162087 & $2.60 \mathrm{E}-48$ \\
\hline ERIC1_1c00990 & M16B & MER071223 & $9.30 \mathrm{E}-40$ \\
\hline ERIC1_1c00990 & M16B & MER084990 & $4.40 \mathrm{E}-26$ \\
\hline ERIC1_1c01000 & M16B & MER013711 & $3.90 \mathrm{E}-85$ \\
\hline ERIC1_1c01150 & M19 & MER013425 & $1.30 \mathrm{E}-51$ \\
\hline ERIC1_1c10510 & M20A & MER173790 & $3.80 \mathrm{E}-119$ \\
\hline ERIC1_1c21340 & M20A & MER001361 & $1.80 \mathrm{E}-89$ \\
\hline ERIC1_1c25270 & M20B & MER001421 & $6.70 \mathrm{E}-121$ \\
\hline ERIC1_2c04520 & M20B & MER028941 & $2.40 \mathrm{E}-129$ \\
\hline
\end{tabular}


Table S1 continued

\begin{tabular}{|c|c|c|c|}
\hline Gene ID & MEROPS family [1] & MEROPS entry & E-value* \\
\hline ERIC1_1c04100 & M20D & MER005163 & $2.50 \mathrm{E}-10$ \\
\hline ERIC1_1c07350 & M20D & MER081890 & $4.50 \mathrm{E}-69$ \\
\hline ERIC1_2c05580 & M20D & MER005163 & $7.00 \mathrm{E}-91$ \\
\hline ERIC1_4c00410 & M20D & MER180918 & $3.10 \mathrm{E}-31$ \\
\hline ERIC1_4c00420 & M20D & MER180918 & $6.50 \mathrm{E}-26$ \\
\hline ERIC1_1c26460 & M22 & MER145515 & $4.20 \mathrm{E}-78$ \\
\hline ERIC1_1c29610 & M22 & MER038778 & $1.30 \mathrm{E}-26$ \\
\hline ERIC1_1c29630 & M22 & MER001274 & $3.10 \mathrm{E}-74$ \\
\hline ERIC1_1c11040 & M23B & MER083041 & $6.30 \mathrm{E}-05$ \\
\hline ERIC1_1c19070 & M23B & MER145504 & $1.30 \mathrm{E}-05$ \\
\hline ERIC1_1c25750 & M23B & MER019259 & $1.20 \mathrm{E}-29$ \\
\hline ERIC1_1c26840 & M23B & MER158191 & $1.50 \mathrm{E}-14$ \\
\hline ERIC1_1c27450 & M23B & MER116068 & $5.90 \mathrm{E}-50$ \\
\hline ERIC1_1c32440 & M23B & MER145504 & $5.40 \mathrm{E}-08$ \\
\hline ERIC1_1c32440 & M23B & MER145504 & $5.50 \mathrm{E}-05$ \\
\hline ERIC1_1c36830 & M23B & MER088463 & $6.30 \mathrm{E}-14$ \\
\hline ERIC1_2c07120 & M23B & MER021826 & 4.20E-61 \\
\hline ERIC1_3c00550 & M23B & MER083041 & 4.60E-06 \\
\hline ERIC1_1c16700 & M24A & MER001243 & $6.20 \mathrm{E}-58$ \\
\hline ERIC1_1c23010 & M24A & MER001243 & $2.90 \mathrm{E}-62$ \\
\hline ERIC1_1c01380 & M24B & MER004931 & $2.00 \mathrm{E}-54$ \\
\hline ERIC1_2c03980 & M24B & MER004931 & $4.40 \mathrm{E}-67$ \\
\hline ERIC1_2c06360 & M24B & MER004931 & $4.30 \mathrm{E}-16$ \\
\hline ERIC1_1c07010 & M29 & MER001285 & $1.10 \mathrm{E}-12$ \\
\hline ERIC1_1c31500 & M29 & MER001287 & $1.70 \mathrm{E}-134$ \\
\hline ERIC1_1c14980 & M32 & MER001186 & $3.00 \mathrm{E}-106$ \\
\hline ERIC1_1c21750 & M34 & MER001345 & 8.40E-09 \\
\hline ERIC1_1c29960 & M34 & MER001345 & $9.70 \mathrm{E}-12$ \\
\hline ERIC1_1c36220 & M34 & MER001345 & 8.80E-12 \\
\hline ERIC1_1c10560 & M38 & MER005767 & $1.00 \mathrm{E}-24$ \\
\hline ERIC1_1c10560 & M38 & MER005767 & $8.00 \mathrm{E}-14$ \\
\hline ERIC1_1c18770 & M38 & MER033184 & 8.00E-37 \\
\hline ERIC1_2c01690 & M38 & MER037714 & $2.20 \mathrm{E}-27$ \\
\hline ERIC1_1c23810 & M41 & MER002602 & $3.50 \mathrm{E}-88$ \\
\hline ERIC1_2c01720 & M41 & MER005496 & $7.60 \mathrm{E}-29$ \\
\hline ERIC1_1c07850 & M42 & MER022215 & 1.90E-09 \\
\hline ERIC1_1c00710 & M50B & MER004469 & $1.00 \mathrm{E}-13$ \\
\hline ERIC1_1c00710 & M50B & MER004480 & $3.40 \mathrm{E}-13$ \\
\hline ERIC1_1c32730 & M50B & MER038874 & $1.40 \mathrm{E}-08$ \\
\hline ERIC1_1c36840 & M50B & MER002454 & $9.30 \mathrm{E}-40$ \\
\hline ERIC1_4c00400 & M50B & MER004466 & $1.20 \mathrm{E}-05$ \\
\hline ERIC1_1c04930 & M56 & MER014140 & $1.50 \mathrm{E}-05$ \\
\hline ERIC1_1c29890 & M60 & MER042489 & $1.20 \mathrm{E}-07$ \\
\hline ERIC1_1c35200 & M60 & MER042489 & $2.70 \mathrm{E}-36$ \\
\hline ERIC1_1c35200 & M60 & MER042489 & $4.60 \mathrm{E}-31$ \\
\hline
\end{tabular}


Table S1 continued

\begin{tabular}{|c|c|c|c|}
\hline Gene ID & MEROPS family [1] & MEROPS entry & E-value* \\
\hline ERIC1_1c35210 & M60 & MER042489 & $6.90 \mathrm{E}-178$ \\
\hline ERIC1_1c35220 & M60 & MER042489 & $1.10 \mathrm{E}-18$ \\
\hline ERIC1_1c37500 & M60 & MER042489 & $2.20 \mathrm{E}-170$ \\
\hline ERIC1_1c37500 & M60 & MER042489 & $6.90 \mathrm{E}-35$ \\
\hline ERIC1_1c37520 & M60 & MER042489 & $4.00 \mathrm{E}-36$ \\
\hline ERIC1_1c10380 & M78 & MER144929 & $1.60 \mathrm{E}-10$ \\
\hline ERIC1_1c17570 & M78 & MER144929 & 4.10E-09 \\
\hline ERIC1_1c03250 & S01B & MER102301 & $4.90 \mathrm{E}-43$ \\
\hline ERIC1_1c33060 & S01B & MER079071 & $2.30 \mathrm{E}-68$ \\
\hline ERIC1_1c25680 & S01X & MER038350 & $2.00 \mathrm{E}-52$ \\
\hline ERIC1_1c05410 & S08A & MER020842 & $1.50 \mathrm{E}-99$ \\
\hline ERIC1_1c11210 & S08A & MER166197 & $2.50 \mathrm{E}-99$ \\
\hline ERIC1_1c19210 & S08A & MER025143 & $9.30 \mathrm{E}-40$ \\
\hline ERIC1_1c21520 & S08A & MER020842 & $6.80 \mathrm{E}-87$ \\
\hline ERIC1_1c26800 & S08A & MER166197 & $1.70 \mathrm{E}-65$ \\
\hline ERIC1_1c28090 & S08A & MER081072 & $1.50 \mathrm{E}-65$ \\
\hline ERIC1_1c28850 & S08A & MER168175 & $2.00 \mathrm{E}-28$ \\
\hline ERIC1_1c30570 & S08A & MER000310 & $2.30 \mathrm{E}-51$ \\
\hline ERIC1_1c30580 & S08A & MER055152 & $9.60 \mathrm{E}-10$ \\
\hline ERIC1_1c33350 & S08A & MER090388 & $8.00 \mathrm{E}-57$ \\
\hline ERIC1_2c05040 & S08A & MER024807 & $6.40 \mathrm{E}-26$ \\
\hline ERIC1_1c00030 & S08X & MER082970 & $3.10 \mathrm{E}-39$ \\
\hline ERIC1_1c13520 & S08X & MER082970 & $3.30 \mathrm{E}-25$ \\
\hline ERIC1_1c39150 & S08X & MER082970 & $8.00 \mathrm{E}-41$ \\
\hline ERIC1_1c12960 & S09C & MER080940 & $2.90 \mathrm{E}-05$ \\
\hline ERIC1_2c06050 & S09C & MER074338 & $5.90 \mathrm{E}-18$ \\
\hline ERIC1_1c09950 & S09X & MER030913 & $2.80 \mathrm{E}-58$ \\
\hline ERIC1_1c10430 & S09X & MER031565 & $1.90 \mathrm{E}-06$ \\
\hline ERIC1_1c19980 & S09X & MER030913 & $2.60 \mathrm{E}-33$ \\
\hline ERIC1_1c25010 & S09X & MER030913 & $5.40 \mathrm{E}-23$ \\
\hline ERIC1_1c05560 & $\mathrm{S} 11$ & MER137663 & $1.30 \mathrm{E}-47$ \\
\hline ERIC1_1c26220 & $\mathrm{S} 11$ & MER028985 & $4.00 \mathrm{E}-89$ \\
\hline ERIC1_2c00510 & $\mathrm{S} 11$ & MER040501 & $8.90 \mathrm{E}-123$ \\
\hline ERIC1_2c00770 & $\mathrm{S} 11$ & MER137663 & $2.60 \mathrm{E}-105$ \\
\hline ERIC1_1c02000 & S12 & MER065584 & $1.00 \mathrm{E}-25$ \\
\hline ERIC1_1c04210 & S12 & MER028999 & $4.60 \mathrm{E}-68$ \\
\hline ERIC1_1c21650 & S12 & MER041576 & $4.30 \mathrm{E}-44$ \\
\hline ERIC1_1c00950 & S14 & MER020357 & $1.10 \mathrm{E}-59$ \\
\hline ERIC1_1c08120 & $\mathrm{S} 14$ & MER085009 & $8.40 \mathrm{E}-62$ \\
\hline ERIC1_1c12100 & S14 & MER085009 & $5.20 \mathrm{E}-17$ \\
\hline ERIC1_1c12110 & S14 & MER085009 & $5.40 \mathrm{E}-39$ \\
\hline ERIC1_1c28670 & S14 & MER125203 & $2.30 \mathrm{E}-87$ \\
\hline ERIC1_1c33770 & S14 & MER085009 & $1.20 \mathrm{E}-33$ \\
\hline ERIC1_1c35840 & S14 & MER085009 & $2.80 \mathrm{E}-60$ \\
\hline ERIC1_1c36400 & S14 & MER125203 & $4.30 \mathrm{E}-80$ \\
\hline
\end{tabular}


Table S1 continued

\begin{tabular}{|c|c|c|c|}
\hline Gene ID & MEROPS family [1] & MEROPS entry & E-value* \\
\hline ERIC1_1c00150 & S16 & MER155619 & $4.10 \mathrm{E}-91$ \\
\hline ERIC1_1c23530 & S16 & MER170752 & $6.40 \mathrm{E}-98$ \\
\hline ERIC1_1c36450 & S16 & MER058049 & $1.70 \mathrm{E}-83$ \\
\hline ERIC1_3c00660 & S16 & MER048375 & $2.50 \mathrm{E}-21$ \\
\hline ERIC1_1c09510 & S24 & MER029010 & $3.90 \mathrm{E}-08$ \\
\hline ERIC1_1c13670 & S24 & MER029010 & $9.70 \mathrm{E}-46$ \\
\hline ERIC1_1c14910 & S24 & MER119211 & $2.00 \mathrm{E}-18$ \\
\hline ERIC1_1c30680 & S24 & MER140304 & $7.60 \mathrm{E}-14$ \\
\hline ERIC1_1c33440 & S24 & MER140304 & $6.50 \mathrm{E}-45$ \\
\hline ERIC1_1c34850 & S24 & MER117873 & $5.20 \mathrm{E}-17$ \\
\hline ERIC1_1c39370 & S24 & MER123765 & $3.10 \mathrm{E}-06$ \\
\hline ERIC1_3c00900 & S24 & MER022307 & $3.90 \mathrm{E}-13$ \\
\hline ERIC1_1c32390 & S26A & MER055807 & $1.10 \mathrm{E}-42$ \\
\hline ERIC1_2c03250 & S26A & MER028421 & $1.50 \mathrm{E}-27$ \\
\hline ERIC1_3c00460 & S26A & MER028421 & $2.10 \mathrm{E}-37$ \\
\hline ERIC1_1c02720 & S33 & MER031617 & $3.60 \mathrm{E}-11$ \\
\hline ERIC1_2c03310 & S33 & MER044641 & $1.20 \mathrm{E}-11$ \\
\hline ERIC1_2c03310 & S33 & MER045883 & $2.70 \mathrm{E}-07$ \\
\hline ERIC1_2c06700 & S33 & MER036066 & $3.20 \mathrm{E}-09$ \\
\hline ERIC1_1c27460 & S41A & MER105195 & 7.10E-93 \\
\hline ERIC1_2c02580 & S41A & MER123543 & $6.40 \mathrm{E}-12$ \\
\hline ERIC1_2c04280 & S55 & MER003459 & 4.70E-92 \\
\hline ERIC1_1c31400 & S58 & MER164925 & $4.90 \mathrm{E}-26$ \\
\hline ERIC1_1c31410 & S58 & MER164925 & $3.50 \mathrm{E}-21$ \\
\hline ERIC1_1c29680 & S66 & MER025441 & $2.40 \mathrm{E}-17$ \\
\hline ERIC1_1c00240 & T01B & MER001626 & $4.80 \mathrm{E}-60$ \\
\hline ERIC1_1c27360 & T05 & MER011829 & $6.30 \mathrm{E}-41$ \\
\hline ERIC1_1c10740 & U04 & MER001293 & $2.20 \mathrm{E}-26$ \\
\hline ERIC1_1c37420 & U32 & MER117225 & $6.50 \mathrm{E}-45$ \\
\hline ERIC1_1c37430 & U32 & MER141870 & $2.40 \mathrm{E}-110$ \\
\hline ERIC1_1c06390 & U35 & MER120958 & $2.50 \mathrm{E}-25$ \\
\hline ERIC1_1c24100 & U57 & MER120195 & $7.10 \mathrm{E}-57$ \\
\hline ERIC1_1c00490 & U68 & MER123660 & $6.30 \mathrm{E}-60$ \\
\hline ERIC1_3c00420 & U68 & MER187143 & $5.00 \mathrm{E}-05$ \\
\hline
\end{tabular}

*an $\mathrm{E}$ value of e-04 or less being considered significant

\section{Reference:}

1. Rawlings N.D., Morton F.R. (2008). The MEROPS batch Blast: a tool to detect peptidases and their non-peptidase homologues in a genome. Biochimie 90: 243259. 
Table S2. Peptidases identified and classified in the genome of $P$. larvae strain DSM 25430.

\begin{tabular}{|c|c|c|c|}
\hline Gene ID & MEROPS family [1] & MEROPS entry & E-value* \\
\hline ERIC2_c24460 & A08 & MER070683 & $2.00 \mathrm{E}-12$ \\
\hline ERIC2_c25740 & $\mathrm{A} 25$ & MER166302 & $7.70 \mathrm{E}-86$ \\
\hline ERIC2_c02380 & $\mathrm{C} 26$ & MER066434 & $2.30 \mathrm{E}-08$ \\
\hline ERIC2_c07940 & $\mathrm{C} 26$ & MER061258 & $1.40 \mathrm{E}-61$ \\
\hline ERIC2_c24400 & $\mathrm{C} 26$ & MER146598 & $3.10 \mathrm{E}-89$ \\
\hline ERIC2_c39480 & $\mathrm{C} 26$ & MER134379 & $3.90 \mathrm{E}-66$ \\
\hline ERIC2_c06210 & $\mathrm{C} 40$ & MER003807 & $6.00 \mathrm{E}-18$ \\
\hline ERIC2_c07300 & $\mathrm{C} 40$ & MER002450 & $4.50 \mathrm{E}-33$ \\
\hline ERIC2_c08860 & $\mathrm{C} 40$ & MER003807 & $1.10 \mathrm{E}-44$ \\
\hline ERIC2_c34370 & $\mathrm{C} 40$ & MER003805 & $7.20 \mathrm{E}-28$ \\
\hline ERIC2_c38180 & $\mathrm{C} 40$ & MER003805 & $1.40 \mathrm{E}-27$ \\
\hline ERIC2_c07830 & $\mathrm{C} 44$ & MER020221 & $4.90 \mathrm{E}-28$ \\
\hline ERIC2_c08270 & $\mathrm{C} 44$ & MER004101 & $2.20 \mathrm{E}-64$ \\
\hline ERIC2_c18310 & $\mathrm{C} 44$ & MER033254 & $1.40 \mathrm{E}-20$ \\
\hline ERIC2_c28690 & $\mathrm{C} 44$ & MER033254 & $1.20 \mathrm{E}-16$ \\
\hline ERIC2_c29880 & $\mathrm{C} 44$ & MER033254 & $8.50 \mathrm{E}-20$ \\
\hline ERIC2_c37770 & C44 & MER003327 & $1.60 \mathrm{E}-60$ \\
\hline ERIC2_c23100 & C56 & MER002455 & $3.90 \mathrm{E}-32$ \\
\hline ERIC2_c41060 & C56 & MER031432 & $2.70 \mathrm{E}-11$ \\
\hline ERIC2_c29130 & $\mathrm{C} 82$ & MER076166 & $2.20 \mathrm{E}-08$ \\
\hline ERIC2_c31230 & $\mathrm{C} 82$ & MER076166 & $5.70 \mathrm{E}-05$ \\
\hline ERIC2_c39100 & M01 & MER055808 & $1.90 \mathrm{E}-29$ \\
\hline ERIC2_c11660 & M03B & MER001163 & $6.70 \mathrm{E}-68$ \\
\hline ERIC2_c30240 & M03B & MER084913 & $1.10 \mathrm{E}-63$ \\
\hline ERIC2_c17790 & M04 & MER001026 & 5.10E-104 \\
\hline ERIC2_c23330 & M04 & MER001026 & $5.20 \mathrm{E}-87$ \\
\hline ERIC2_c25850 & M04 & MER001026 & $5.20 \mathrm{E}-87$ \\
\hline ERIC2_c27330 & M06 & MER001164 & $7.00 \mathrm{E}-127$ \\
\hline ERIC2_c40370 & M09B & MER001417 & $4.40 \mathrm{E}-120$ \\
\hline ERIC2_c11410 & M14C & MER001505 & $1.90 \mathrm{E}-78$ \\
\hline ERIC2_c36940 & M15B & MER084164 & $1.30 \mathrm{E}-49$ \\
\hline ERIC2_c21010 & M16B & MER013711 & $2.00 \mathrm{E}-84$ \\
\hline ERIC2_c21020 & M16B & MER084990 & $1.00 \mathrm{E}-25$ \\
\hline ERIC2_c21020 & M16B & MER071223 & $9.30 \mathrm{E}-40$ \\
\hline ERIC2_c21140 & M16B & MER162087 & $2.00 \mathrm{E}-48$ \\
\hline ERIC2_c21140 & M16B & MER142621 & $6.10 \mathrm{E}-90$ \\
\hline ERIC2_c20860 & M19 & MER013425 & $1.30 \mathrm{E}-51$ \\
\hline ERIC2_c05420 & M20A & MER001361 & $1.80 \mathrm{E}-89$ \\
\hline ERIC2_c24360 & M20A & MER173790 & $3.80 \mathrm{E}-119$ \\
\hline ERIC2_c01850 & M20B & MER001421 & $6.70 \mathrm{E}-121$ \\
\hline ERIC2_c12590 & M20B & MER028941 & $2.40 \mathrm{E}-129$ \\
\hline ERIC2_c15490 & M20D & MER081890 & $2.00 \mathrm{E}-67$ \\
\hline ERIC2_c18240 & M20D & MER005163 & $2.50 \mathrm{E}-10$ \\
\hline
\end{tabular}


Table S2 continued

\begin{tabular}{|c|c|c|c|}
\hline Gene ID & MEROPS family [1] & MEROPS entry & E-value* \\
\hline ERIC2_c22920 & M20D & MER180918 & $3.10 \mathrm{E}-31$ \\
\hline ERIC2_c22921 & M20D & MER180918 & $6.50 \mathrm{E}-26$ \\
\hline ERIC2_c00360 & M22 & MER145515 & $4.80 \mathrm{E}-77$ \\
\hline ERIC2_c03500 & M22 & MER038778 & $1.30 \mathrm{E}-26$ \\
\hline ERIC2_c03520 & M22 & MER001274 & $3.10 \mathrm{E}-74$ \\
\hline ERIC2_c00710 & M23B & MER158191 & $1.10 \mathrm{E}-14$ \\
\hline ERIC2_c01320 & M23B & MER116068 & $5.90 \mathrm{E}-50$ \\
\hline ERIC2_c01580 & M23B & MER019259 & $1.20 \mathrm{E}-29$ \\
\hline ERIC2_c22020 & M23B & MER083041 & $4.60 \mathrm{E}-06$ \\
\hline ERIC2_c24890 & M23B & MER083041 & $8.60 \mathrm{E}-05$ \\
\hline ERIC2_c28230 & M23B & MER088463 & $6.30 \mathrm{E}-14$ \\
\hline ERIC2_c30340 & M23B & MER021826 & $4.20 \mathrm{E}-61$ \\
\hline ERIC2_c35810 & M23B & MER145504 & $5.40 \mathrm{E}-08$ \\
\hline ERIC2_c35810 & M23B & MER166256 & $4.50 \mathrm{E}-06$ \\
\hline ERIC2_c11500 & M24A & MER001243 & $6.20 \mathrm{E}-58$ \\
\hline ERIC2_c38280 & M24A & MER001243 & $1.90 \mathrm{E}-61$ \\
\hline ERIC2_c20680 & M24B & MER004931 & $2.00 \mathrm{E}-54$ \\
\hline ERIC2_c31110 & M24B & MER004931 & $9.30 \mathrm{E}-17$ \\
\hline ERIC2_c33050 & M24B & MER004931 & $4.40 \mathrm{E}-67$ \\
\hline ERIC2_c15870 & M29 & MER001285 & $1.10 \mathrm{E}-12$ \\
\hline ERIC2_c36840 & M29 & MER001287 & $2.20 \mathrm{E}-134$ \\
\hline ERIC2_c27270 & M32 & MER001186 & 2.30E-106 \\
\hline ERIC2_c05310 & M34 & MER001345 & $2.00 \mathrm{E}-12$ \\
\hline ERIC2_c28760 & M34 & MER001345 & $6.00 \mathrm{E}-08$ \\
\hline ERIC2_c07350 & M38 & MER033184 & $8.00 \mathrm{E}-37$ \\
\hline ERIC2_c14070 & M38 & MER037714 & $2.20 \mathrm{E}-27$ \\
\hline ERIC2_c24410 & M38 & MER005767 & $1.00 \mathrm{E}-24$ \\
\hline ERIC2_c24410 & M38 & MER005767 & $8.00 \mathrm{E}-14$ \\
\hline ERIC2_c14100 & M41 & MER005496 & $1.10 \mathrm{E}-28$ \\
\hline ERIC2_c39570 & M41 & MER002602 & $3.50 \mathrm{E}-88$ \\
\hline ERIC2_c21290 & M50B & MER004469 & $1.00 \mathrm{E}-13$ \\
\hline ERIC2_c21290 & M50B & MER004480 & $3.40 \mathrm{E}-13$ \\
\hline ERIC2_c22910 & M50B & MER004466 & 8.90E-06 \\
\hline ERIC2_c28220 & M50B & MER002454 & $7.00 \mathrm{E}-38$ \\
\hline ERIC2_c35500 & M50B & MER038874 & $1.40 \mathrm{E}-08$ \\
\hline ERIC2_c08430 & M78 & MER144929 & $2.50 \mathrm{E}-11$ \\
\hline ERIC2_c24210 & M78 & MER144929 & $1.60 \mathrm{E}-10$ \\
\hline ERIC2_c01340 & S01B & MER087818 & $8.30 \mathrm{E}-05$ \\
\hline ERIC2_c35160 & S01B & MER079071 & $2.30 \mathrm{E}-68$ \\
\hline ERIC2_c01640 & S01X & MER038350 & $1.20 \mathrm{E}-52$ \\
\hline ERIC2_c00670 & S08A & MER166197 & $3.00 \mathrm{E}-65$ \\
\hline ERIC2_c04380 & S08A & MER000310 & $2.30 \mathrm{E}-51$ \\
\hline ERIC2_c04390 & S08A & MER138281 & 8.90E-06 \\
\hline ERIC2_c09000 & S08A & MER025143 & $9.30 \mathrm{E}-40$ \\
\hline ERIC2_c34730 & S08A & MER090388 & $8.00 \mathrm{E}-57$ \\
\hline
\end{tabular}


Table S2 continued

\begin{tabular}{|c|c|c|c|}
\hline Gene ID & MEROPS family [1] & MEROPS entry & E-value* \\
\hline ERIC2_c39030 & S08A & MER081072 & $1.50 \mathrm{E}-65$ \\
\hline ERIC2_c29670 & S09C & MER080940 & $2.90 \mathrm{E}-05$ \\
\hline ERIC2_c31490 & S09C & MER074338 & $5.90 \mathrm{E}-18$ \\
\hline ERIC2_c07680 & S09X & MER030913 & $2.60 \mathrm{E}-33$ \\
\hline ERIC2_c23880 & S09X & MER030913 & $2.80 \mathrm{E}-58$ \\
\hline ERIC2_c24270 & S09X & MER031565 & $1.90 \mathrm{E}-06$ \\
\hline ERIC2_c40780 & S09X & MER030913 & 4.70E-23 \\
\hline ERIC2_c00130 & S11 & MER028985 & $4.00 \mathrm{E}-89$ \\
\hline ERIC2_c12800 & $\mathrm{S} 11$ & MER040501 & $2.30 \mathrm{E}-123$ \\
\hline ERIC2_c13110 & $\mathrm{S} 11$ & MER137663 & $2.60 \mathrm{E}-105$ \\
\hline ERIC2_c05200 & $\mathrm{S} 12$ & MER041576 & $1.10 \mathrm{E}-29$ \\
\hline ERIC2_c18130 & $\mathrm{S} 12$ & MER028999 & $4.60 \mathrm{E}-68$ \\
\hline ERIC2_c20000 & $\mathrm{S} 12$ & MER065584 & $1.00 \mathrm{E}-25$ \\
\hline ERIC2_c02560 & $\mathrm{S} 14$ & MER125203 & $2.30 \mathrm{E}-87$ \\
\hline ERIC2_c21060 & $\mathrm{S} 14$ & MER020357 & $1.10 \mathrm{E}-59$ \\
\hline ERIC2_c28620 & $\mathrm{S} 14$ & MER125203 & $1.50 \mathrm{E}-80$ \\
\hline ERIC2_c21870 & S16 & MER155619 & $1.60 \mathrm{E}-94$ \\
\hline ERIC2_c22120 & $\mathrm{S} 16$ & MER048375 & $7.90 \mathrm{E}-21$ \\
\hline ERIC2_c28570 & $\mathrm{S} 16$ & MER058049 & $1.70 \mathrm{E}-83$ \\
\hline ERIC2_c28580 & S16 & MER164590 & $1.70 \mathrm{E}-79$ \\
\hline ERIC2_c28580 & $\mathrm{S} 16$ & MER155619 & $1.60 \mathrm{E}-07$ \\
\hline ERIC2_c38790 & $\mathrm{S} 16$ & MER170752 & $6.40 \mathrm{E}-98$ \\
\hline ERIC2_c04500 & $\mathrm{S} 24$ & MER140304 & $7.60 \mathrm{E}-14$ \\
\hline ERIC2_c25960 & $\mathrm{S} 24$ & MER029010 & $9.70 \mathrm{E}-46$ \\
\hline ERIC2_c27180 & S24 & MER119211 & $2.00 \mathrm{E}-18$ \\
\hline ERIC2_c21930 & S26A & MER028421 & $7.00 \mathrm{E}-38$ \\
\hline ERIC2_c31810 & S26A & MER028421 & $1.50 \mathrm{E}-27$ \\
\hline ERIC2_c35880 & S26A & MER055807 & $1.10 \mathrm{E}-42$ \\
\hline ERIC2_c18550 & S33 & MER036050 & $8.50 \mathrm{E}-21$ \\
\hline ERIC2_c30770 & S33 & MER036066 & $5.30 \mathrm{E}-10$ \\
\hline ERIC2_c31750 & S33 & MER044641 & $1.20 \mathrm{E}-11$ \\
\hline ERIC2_c31750 & S33 & MER045883 & $2.70 \mathrm{E}-07$ \\
\hline ERIC2_c01330 & S41A & MER105195 & $2.10 \mathrm{E}-92$ \\
\hline ERIC2_c32750 & S55 & MER003459 & 4.70E-92 \\
\hline ERIC2_c36941 & $\mathrm{S} 58$ & MER164925 & $3.50 \mathrm{E}-21$ \\
\hline ERIC2_c36950 & S58 & MER100239 & $8.40 \mathrm{E}-05$ \\
\hline ERIC2_c36950 & $\mathrm{S} 58$ & MER164925 & $4.90 \mathrm{E}-26$ \\
\hline ERIC2_c03570 & S66 & MER142981 & $4.20 \mathrm{E}-19$ \\
\hline ERIC2_c21760 & T01B & MER001626 & $1.40 \mathrm{E}-59$ \\
\hline ERIC2_c01230 & T05 & MER011829 & $4.80 \mathrm{E}-41$ \\
\hline ERIC2_c24590 & U04 & MER001293 & $2.20 \mathrm{E}-26$ \\
\hline ERIC2_c27630 & U32 & MER117225 & $6.50 \mathrm{E}-45$ \\
\hline ERIC2_c05010 & U32 & MER019303 & $7.20 \mathrm{E}-59$ \\
\hline ERIC2_c05010 & U32 & MER019303 & $1.80 \mathrm{E}-09$ \\
\hline ERIC2_c27620 & U32 & MER117225 & $1.20 \mathrm{E}-111$ \\
\hline
\end{tabular}


Table $\mathrm{S} 2$ continued

\begin{tabular}{llll}
\hline Gene ID & MEROPS family [1] & MEROPS entry & E-value* \\
\hline ERIC2_c39860 & U57 & MER120195 & $7.10 \mathrm{E}-57$ \\
ERIC2_c21510 & U68 & MER123660 & $6.30 \mathrm{E}-60$ \\
ERIC2_c21890 & U68 & MER187143 & $5.00 \mathrm{E}-05$ \\
\hline *
\end{tabular}

*an $\mathrm{E}$ value of e-04 or less being considered significant

\section{Reference:}

1. Rawlings N.D., Morton F.R. (2008). The MEROPS batch Blast: a tool to detect peptidases and their non-peptidase homologues in a genome. Biochimie 90: 243-259. 
Table S3. P. larvae strains used for this study.

\begin{tabular}{lll}
\hline Strain & Source & ERIC genotype \\
\hline ATCC 9545 & ATCC & I \\
DSM 25719 & Honey (dis. col.) & I \\
$02-075$ & Honey (dis. col.) & I \\
$02-081$ & Honey (dis. col.) & I \\
$02-179$ & Honey (dis. col.) & I \\
$02-250$ & Honey (dis. col.) & I \\
$03-119$ & Honey (dis. col.) & I \\
$03-122$ & Honey (dis. col.) & I \\
$03-189$ & Honey (dis. col.) & I \\
$00-1163$ & Honey (dis. col.) & I I \\
$01-1714$ & Honey (dis. col.) & I I \\
$03-016$ & Honey (dis. col.) & I I \\
$03-195$ & Honey (dis. col.) & I I \\
$03-199$ & Honey (dis. col.) & I I \\
$03-200$ & Honey (dis. col.) & I I \\
$03-522$ & Honey (dis. col.) & I I \\
$03-478$ & Honey (dis. col.) & I I \\
$03-518$ & Honey (dis. col.) & I I \\
$03-525$ & Honey (dis. col.) & I I \\
DSM 25430 & Honey (dis. col.) & I I \\
\hline (dis. col.), AFB-diseased colony.
\end{tabular}




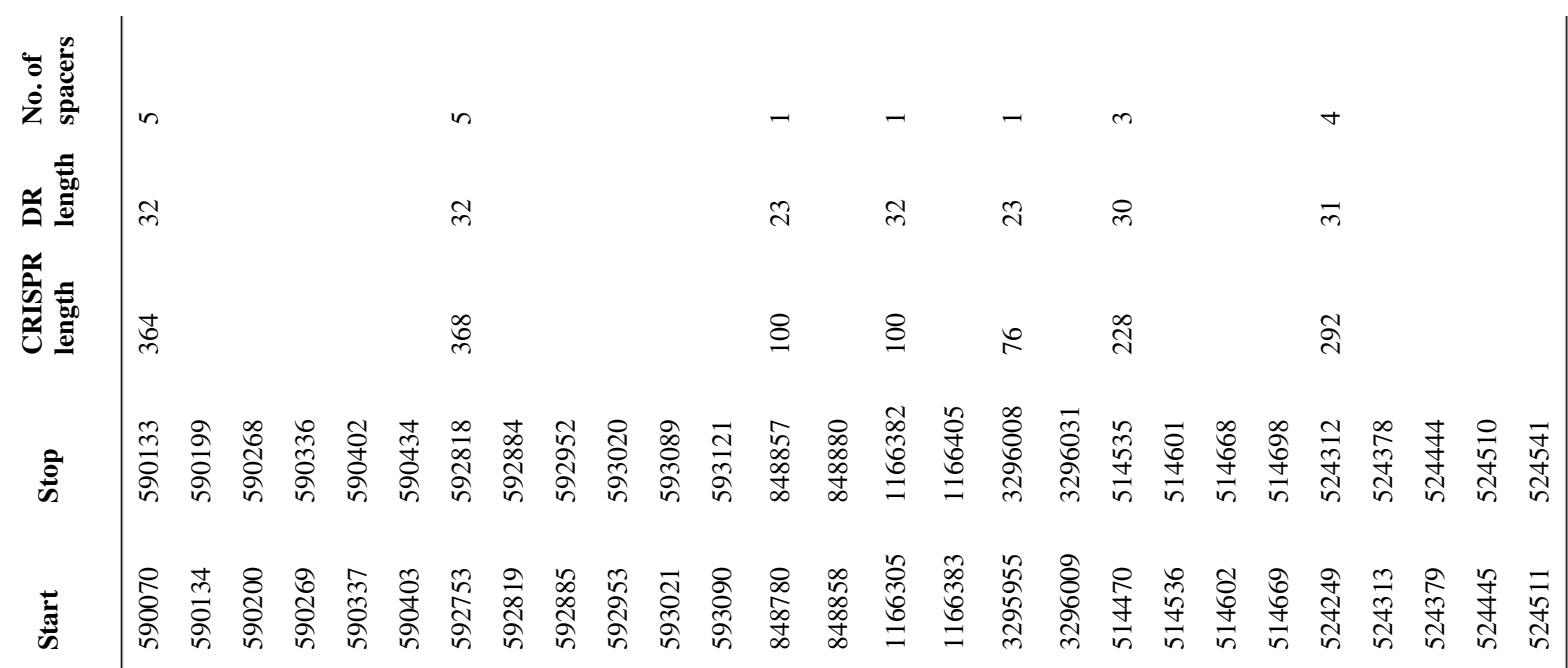

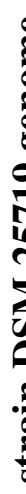
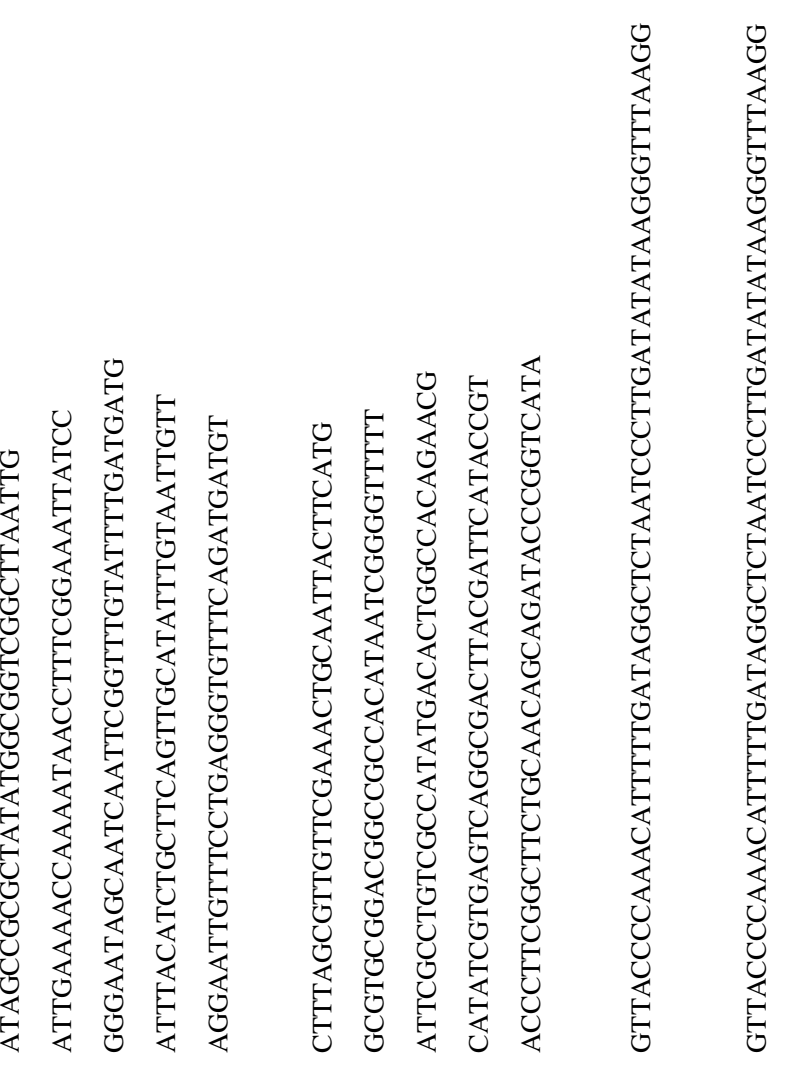

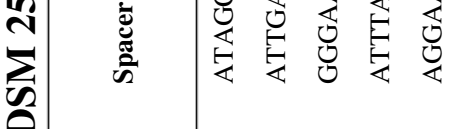

U U U U U U U U U U U U U U U U

\section{还}

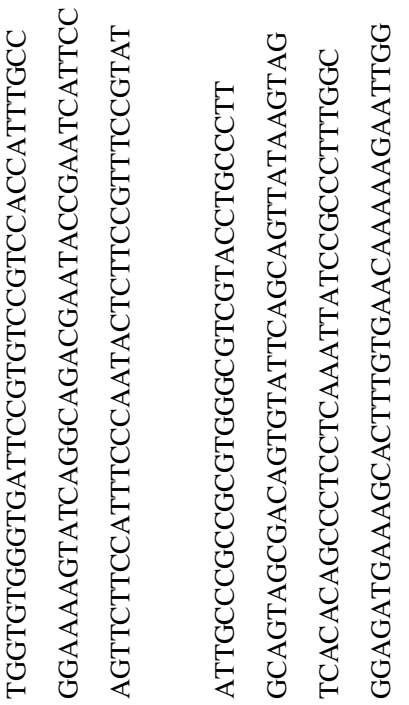

U.

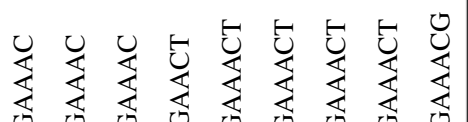

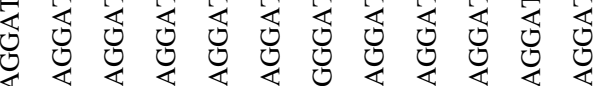

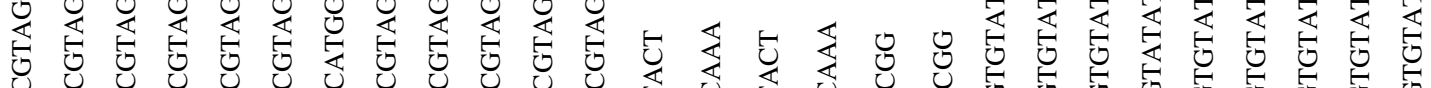
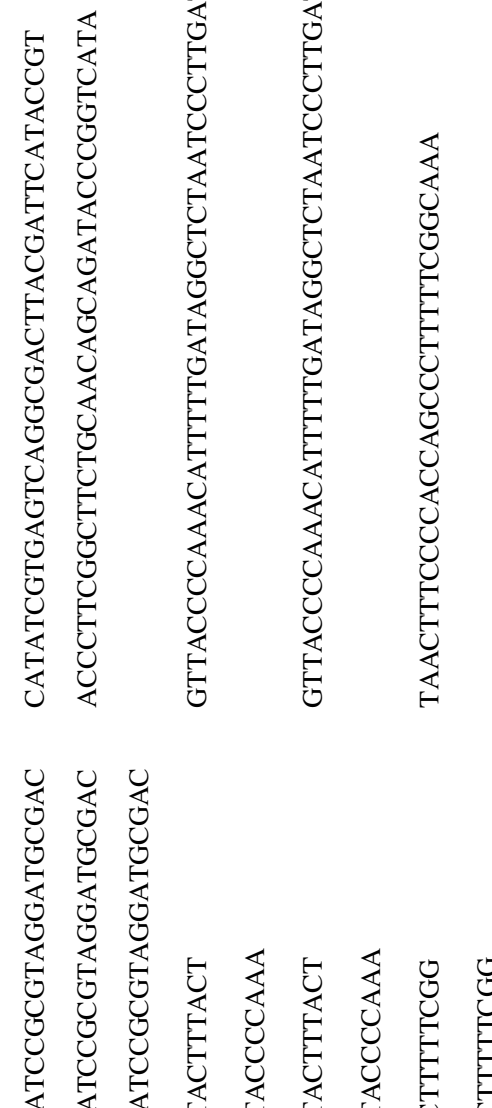

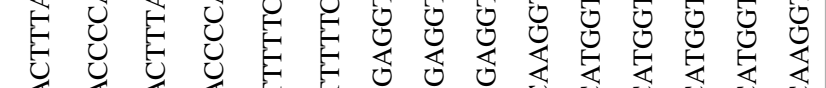

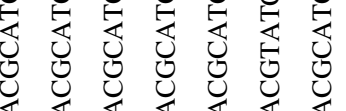

E

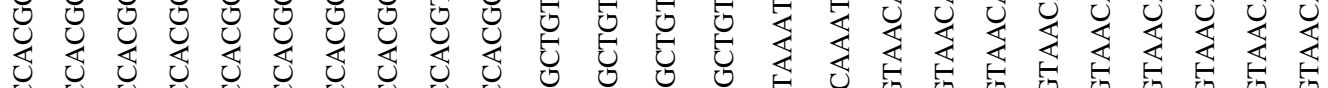
G

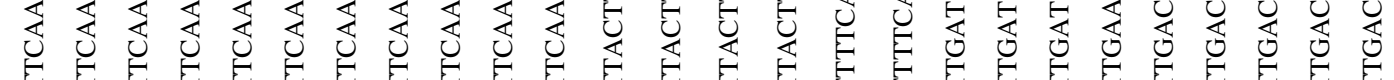




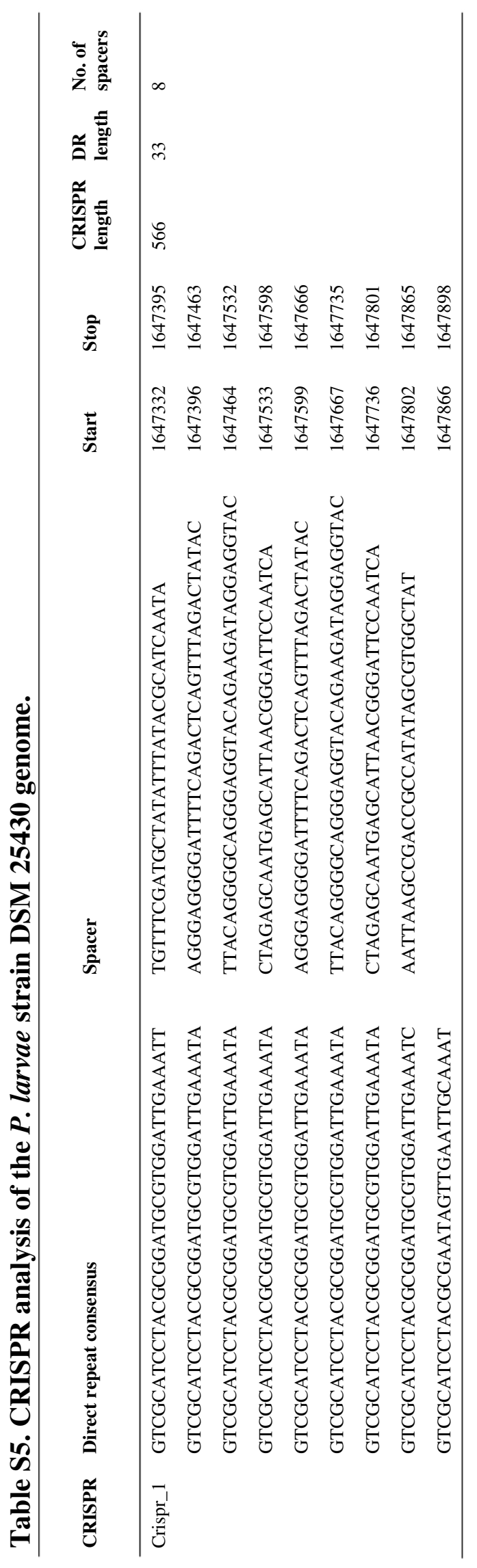




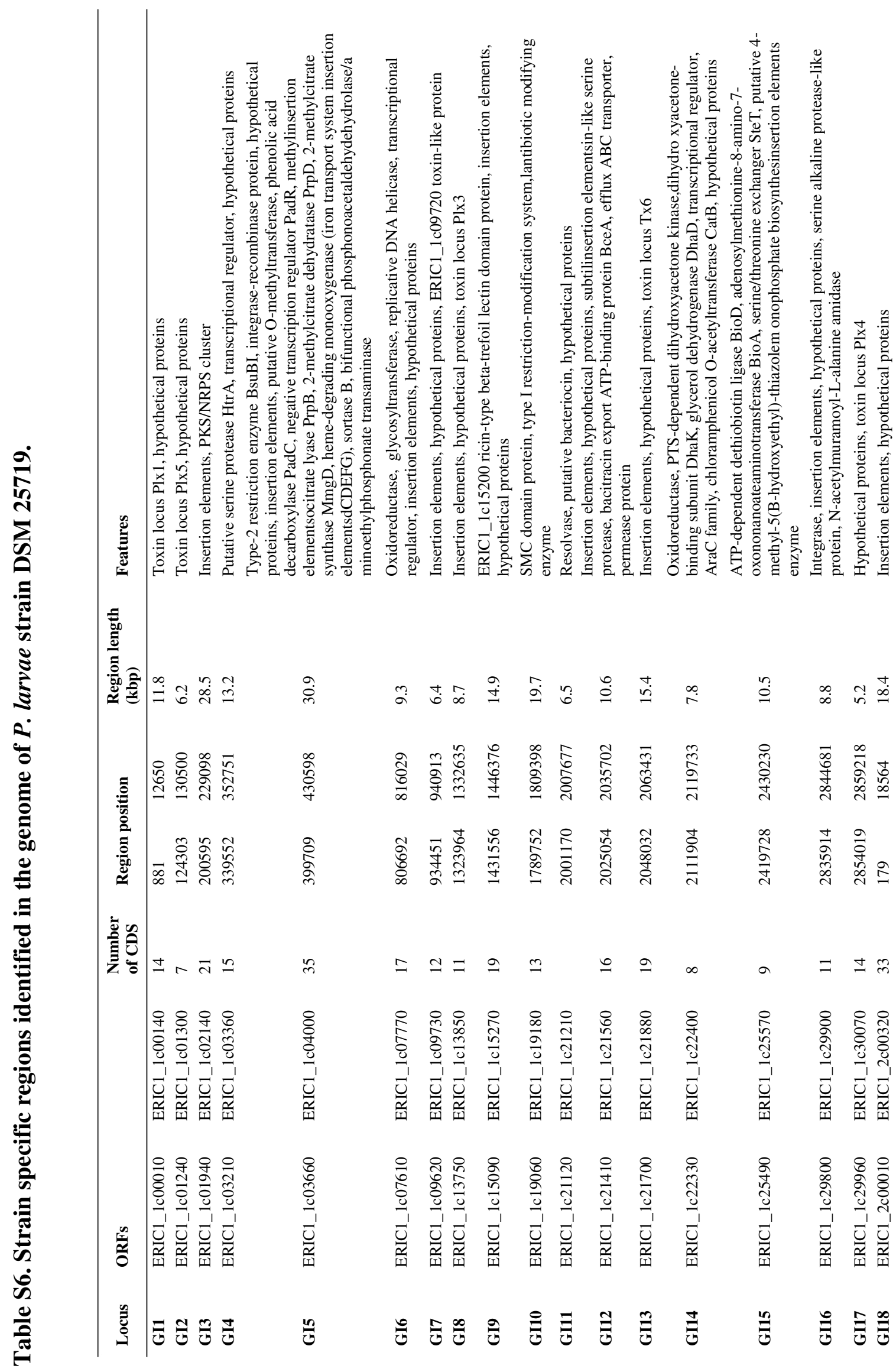




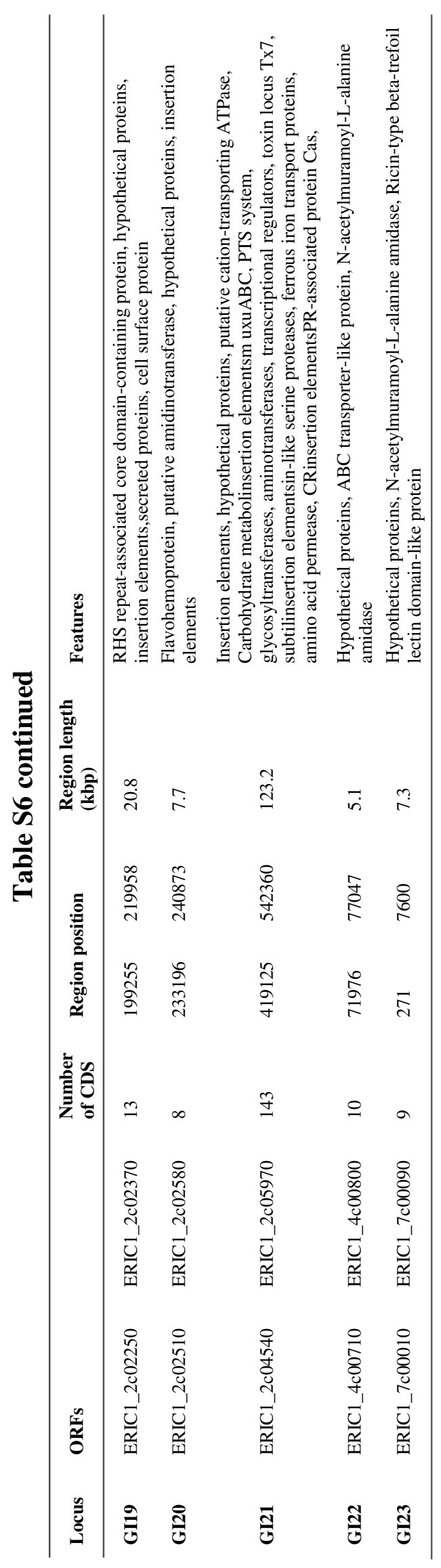




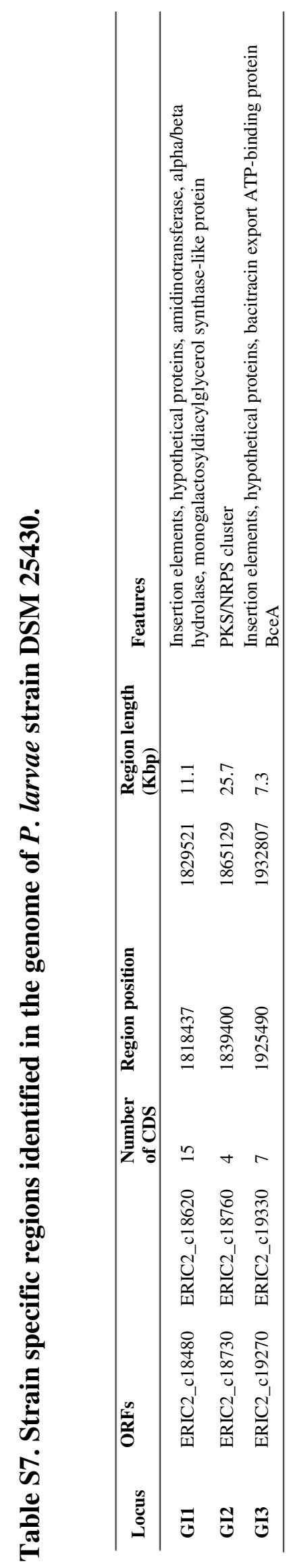




\section{Paenilarvins: Iturin Family Lipopeptides from the Honey Bee Pathogen Paenibacillus larvae}

Sakshi Sood ${ }^{1}$, Heinrich Steinmetz ${ }^{1}$, Hannes Beims ${ }^{2}$, Kathrin I. Mohr ${ }^{1}$, Marc Stadler ${ }^{1}$, Marvin Djukic ${ }^{3}$, Werner von der Ohe ${ }^{4}$, Michael Steinert ${ }^{2}$, Rolf Daniel ${ }^{3}$, Rolf Müller ${ }^{1,5}$

ChemBioChem (2014), Vol. 15, 1947-1955

${ }^{1}$ Abteilung für Mikrobielle Naturstoffe, Helmholtz Zentrum für Infektionsforschung, Braunschweig, Deutschland

${ }^{2}$ Institut für Mikrobiologie, Technische Universität Braunschweig, Braunschweig, Deutschland

${ }^{3}$ Abteilung für Genomische und Angewandte Mikrobiologie \& Göttingen Genomics Laboratory, Institut für Mikrobiologie und Genetik, Georg-August Universität Göttingen, Göttingen, Deutschland

${ }^{4}$ Niedersächsisches Landesamt für Verbraucherschutz und Lebensmittelsicherheit, Celle, Deutschland

${ }^{5}$ Mikrobielle Naturstoffe, Helmholtz Zentrum für Pharmazeutische Forschung Saarland, Abteilung für Pharmazeutische Biotechnologie, Saarland Universität, Saarbrücken, Deutschland

\section{Anteilserklärung}

Idee/Konzept: MS, HS, KM, RD, RM

In silico Analyse des NRPS/PKS-Hybridgenclusters: MD

Aufreinigung der Verbindungen: SS, HS

Durchführung der biologischen Assays und Analyse der Daten: SS, HB, WO, MS

Analyse der chemischen und NMR-Spektroskopie-Daten: HS

Schreiben des Manuskripts: SS, HS 


\title{
Paenilarvins: Iturin Family Lipopeptides from the Honey Bee Pathogen Paenibacillus larvae
}

\author{
Sakshi Sood ${ }_{,}^{[a]}$ Heinrich Steinmetz, ${ }^{[a]}$ Hannes Beims, ${ }^{[\mathrm{d}]}$ Kathrin I. Mohr, ${ }^{[\mathrm{a}]}$ Marc Stadler, ${ }^{[a]}$ \\ Marvin Djukic, ${ }^{[b]}$ Werner von der Ohe, ${ }^{[\mathrm{e}]}$ Michael Steinert, ${ }^{[\mathrm{d}]}$ Rolf Daniel, $^{[\mathrm{b}]}$ and Rolf Müller ${ }^{*[a, c]}$
}

\begin{abstract}
The bacterium Paenibacillus larvae has been extensively studied as it is an appalling honey bee pathogen. In the present work, we screened crude extracts derived from fermentations of $P$. larvae genotypes ERIC I and II for antimicrobial activity, following the detection of four putative secondary metabolite gene clusters that show high sequence homology to known biosynthetic gene clusters for the biosynthesis of antibiotics. Low molecular weight metabolites produced by P. larvae have recently been shown to have toxic effects on honey bee larvae. Moreover, a novel tripeptide, sevadicin, was recently characterized from laboratory cultures of $P$. larvae. In this study,
\end{abstract}

paenilarvins, which are iturinic lipopeptides exhibiting strong antifungal activities, were obtained by bioassay-guided fractionation from cultures of $P$. larvae, genotype ERIC II. Their molecular structures were determined by extensive 2D NMR spectroscopy, high resolution mass spectrometry, and other methods. Paenilarvins are the first antifungal secondary metabolites to be identified from P. larvae. In preliminary experiments, these lipopeptides also affected honey bee larvae and might thus play a role in P. larvae survival and pathogenesis. However, further studies are needed to investigate their function.

\section{Introduction}

American foulbrood (AFB), one of the most catastrophic honey bee (Apis mellifera) epidemics, is a notifiable disease in many countries, and strict laws are enforced for its control. Burning of infected or diseased colonies is considered the most effective control measure against AFB in most countries, thus bringing about huge losses. ${ }^{[1]}$ However, despite being a deleterious and economically significant disease for honey bees, the molecular pathogenesis of AFB remains obscure.

Paenibacillus larvae, a Gram-positive, rod-shaped, sporeforming bacterium, only infects larvae in first instar stage

[a] Dr. S. Sood, H. Steinmetz, Dr. K. I. Mohr, Prof. Dr. M. Stadler, Prof. Dr. R. Müller

Department of Microbial Drugs, Helmholtz Centre for Infection Research Inhoffenstrasse 7, 38124 Braunschweig (Germany)

E-mail: rom@mx-uni.saarland.de

[b] M. Djukic, Prof. Dr. R. Daniel

Department of Genomic and Applied Microbiology

Institute of Microbiology and Genetics, Georg-August University Grisebachstrasse 8, 37077 Göttingen (Germany)

[c] Prof. Dr. R. Müller

Microbial Natural Products

Helmholtz Institute for Pharmaceutical Research Saarland

Department of Pharmaceutical Biotechnology, Saarland University

Campus C2.3, 66123 Saarbrücken (Germany)

[d] H. Beims, Prof. Dr. M. Steinert

Institute of Microbiology, Technichal University Braunschweig

Spielmannstrasse 7, 38106 Braunschweig (Germany)

[e] W. von der Ohe

Lower Saxony State Office for Consumer Protection and Food Safety Institute for Apiculture

Herzogin-Eleonore-Allee 5, 29221 Celle (Germany)

Supporting information for this article is available on the WWW under http://dx.doi.org/10.1002/cbic.201402139. through its spores, and is the etiological agent of AFB. Genotyping of $P$. larvae isolates on the basis of PCR with enterobacterial repetitive intergenic consensus (ERIC) primers identified four genotypes: P. larvae ERIC I-IV..$^{[2]}$ The strains vary in various aspects, most importantly in their level of virulence. ${ }^{[1,3]}$ Strains of genotypes ERIC I and ERIC II are the most common field isolates and account for most of the AFB outbreaks worldwide; very few isolates of ERIC III and ERIC IV have been reported.

Fluorescence in situ hybridization (FISH) with P. larvae-specific $16 \mathrm{~S}$ rRNA targeted oligonucleotide probes was used to study the interaction between the host and the pathogen (genotypes ERICI and II) in detail. ${ }^{[4]}$ This study gave deep insights into the disease progression-from the ingestion of spores of $P$. larvae by the honey bee larvae in contaminated food to the formation of hard scales from dead larval remains. These hard scales are the source of millions of bacterial spores, which can be further transmitted by contaminated equipment or by adult honey bees feeding on contaminated honey.

A number of potential virulence factors have been described to be instrumental in P. larvae pathogenesis in recent years. The secretome of P. larvae has been studied in detail, thus leading to the identification of some of these virulence factors, for example, an enolase that is highly toxic and immunogenic in bees. ${ }^{[5]}$ This pathogen also produces metalloprotease in vivo during bee larvae infection. ${ }^{[6]}$ Both enzymes have been suggested to be involved in larval degradation during and after infection. Moreover, Poppinga et al. recently identified the Slayer protein SpIA as an important virulence factor of $P$. larvae ERIC II. ${ }^{[7]}$ In another study, the P. larvae ERIC I strain was shown to produce $A B$ binary toxins $P \mid x 1$ and $P \mid x 2$ as virulence factors. $^{[8]}$ 
In an attempt to identify all putative virulence genes in $P$. larvae, suppression subtractive hybridization (SSH) was applied in a comparative genomic analysis of $P$. larvae ${ }^{[9]}$ several fragments showing homology to subunits of nonribosomal peptide synthetases (NRPSs) and/or polyketide synthetases (PKSs) synthesizing iturin-family lipopeptides (bacitracin and bacillomycin) were identified in different genotypes. Open reading frames (ORFs) showing close homology to NRPSs for the antibiotics plipastatin and surfactin were observed in another $P$. larvae genome study by a combination of bioinformatics and proteomics analysis. ${ }^{[10]}$ The end products of these multistep biosynthetic complexes are nonribosomal peptides or polyketides, (or their hybrids), with broad structural diversity and biological activities like enzyme inhibition, immunosuppression, as well as antiparasitic agents, bioherbicides, plant growth regulators, biopesticides, bioinsecticides, antitumor agents, and microbial survival and pathogenesis compounds. ${ }^{[11]}$ Schild et al. recently tested the toxicity of undefined secondary metabolites produced by P. larvae against honey bee larvae, and speculated on their roles in pathogenesis. ${ }^{[12]}$ An antibacterial tripeptide, sevadicin, encoded by a NRPS gene cluster was recently characterized from $P$. larvae ERIC $1{ }^{[13]}$

The confirmation of the presence of NRPS and NRPS/PKS hybrid clusters in the genomes of P. larvae DSM 25719 (ERIC I) and DSM 25430 (ERIC II) and their possible role as virulence markers ${ }^{[12,14]}$ prompted us to examine $P$. larvae for the production of secondary metabolites that could be significant virulence factors (or potentially useful in pharmaceutical or agrochemical applications). Here we characterized three novel iturin-type lipopeptides (named paenilarvins A-C) from P. larvae DSM 25430 (ERIC II). We also analyzed the gene cluster putatively responsible for the production of paenilarvins. Interestingly, the new compounds are similar to mojavensin A, a lipopeptide from the marine-derived bacterium Bacillus mojavensis $\mathrm{B}^{0621 \mathrm{~A} .{ }^{[15]}}$

\section{Results and Discussion}

\section{Bioactivity screening of $P$. larvae}

Strains DSM 25719 (ERIC I) and DSM 25430 (ERIC II) were cultured in the presence of an adsorber resin (Amberlite XAD-16). After harvesting, crude methanol extracts of the resin were tested for antimicrobial activity against Gram-positive and Gram-negative bacteria, yeast, and filamentous fungi, in 96well-plate serial dilution assays.

Crude extracts of both strains initially showed weak and non-enrichable activity against Nocardia flava, Staphylococcus aureus, Chromobacterium violaceum, but more prominently against the zygomycete fungus, Mucor hiemalis. The presence of an antibacterial agent in cultures of $P$. larvae was reported by Glinski and Jarosz. ${ }^{[16]}$ However, the antibacterial activity in our extracts was not reproducible, although the antifungal activity against $M$. hiemalis was consistent in extracts from various batches. The crude extract from DSM 25430 was much more bioactive than that from DSM 25719 against $M$. hiemalis
(MIC $2.1 \mu \mathrm{g} \mathrm{mL}^{-1}$ ). This prompted us to investigate the production of antifungal compounds by strain DSM 25430.

Paenibacillus species have been described as significant sources of biologically active secondary metabolites. ${ }^{[17]} A$ number of other entomopathogenic bacteria have also been described as promising sources of secondary metabolites. ${ }^{[18]}$ Therefore, the crude extract from strain DSM 25430 was subjected to bioassay-guided HPLC fractionation and mass spectrometry to detect the molecular masses of the antifungal compounds; this revealed a group of molecules over the range $1069-1112.7 \mathrm{Da}$ in the bioactive fractions. The mass range of the identified compounds was close to that of iturin family compounds (e.g., mycosubtilin and mojavensin produced by Bacillus spp.), ${ }^{[15,19]}$ but a detailed structural analysis was essential for complete characterization.

\section{Isolation and structure elucidation of paenilarvins}

P. larvae DSM 25430 (ERIC II) was fermented on a $5 \mathrm{~L}$ scale with XAD-16 amberlite adsorber resin, which was sieved and eluted with methanol to give a crude extract after fermentation. Successive chromatography of the crude methanol extract on silica gel and a reversed-phase column (guided by antifungal activity against $M$. hiemalis) yielded seven compounds of the paenilarvin group. The most active compounds, paenilarvins $A$ and $B$, were subjected to structure elucidation and biological assays. The complete molecular structures of paenilarvins $A$ and $B$ were determined by mass and NMR (1D and 2D) analysis.

Paenilarvin A (1) was obtained by RP-HPLC as a colorless amorphous powder. The positive HRESIMS spectra presented a molecular ion cluster at $\mathrm{m} / \mathrm{z} 1112.6108[\mathrm{M}+\mathrm{H}]^{+}$, consistent with the molecular formula $\mathrm{C}_{52} \mathrm{H}_{82} \mathrm{~N}_{13} \mathrm{O}_{14}$ (calcd 1112.6099), and supported by the ${ }^{13} \mathrm{C}$ NMR spectrum. The intense IR absorptions between 1600 and $1700 \mathrm{~cm}^{-1}$ and between 3100 and $3400 \mathrm{~cm}^{-1}$ showed the presence of amide $\mathrm{C}=\mathrm{O}$ and $\mathrm{NH}$ groups, respectively. The weak UV bands at 277 and $223 \mathrm{~nm}$ were in good agreement with the spectra of a peptide containing aromatic amino acids. Among all 52 carbons in the signals, the ${ }^{13} \mathrm{C}$ NMR spectrum of 1 displayed 13 amide carbonyl signals (172.7-177.9 ppm) and eight methine carbons (48.5$63.2 \mathrm{ppm}$ ), as would be expected for a small peptide. Correspondingly, the complex ${ }^{1} \mathrm{H}$ NMR spectrum of 1 furnished 17 $\mathrm{H} / \mathrm{D}$ exchangeable protons $(6.75-8.75 \mathrm{ppm})$ and at least eight methine protons $(4.05-5.0 \mathrm{ppm})$. A detailed analysis of the COSY, TOCSY, HSQC, and HMBC data for 1 in $\mathrm{CD}_{3} \mathrm{OH}$ revealed the presence of four asparagine, one tyrosine, one glutamine, and one proline (Scheme 1 and Table 1). Furthermore, the methylene group $\left(\delta_{\mathrm{C}}=44.2, \delta_{\mathrm{H}}=2.49,2.41\right)$ and the methine $\left(\delta_{\mathrm{C}}=48.5, \delta_{\mathrm{H}}=4.15\right)$ adjacent to the doublet of $23 \mathrm{NH}\left(\delta_{\mathrm{H}}=\right.$ $7.49,9.5 \mathrm{~Hz}$ ) were recognized as parts of a long $\beta$-amino-acid. Signals for the methyl doublet $\left(\delta_{\mathrm{C}}=19.7, \delta_{\mathrm{H}}=0.86\right)$ and a methyl triplet $\left(\delta_{\mathrm{C}}=11.8, \delta_{\mathrm{H}}=0.87\right)$ together with the remaining methine $\left(\delta_{\mathrm{C}}=35.7, \delta_{\mathrm{H}}=1.29\right)$ and methylene signals $\left(\delta_{\mathrm{C}}=\right.$ $\left.30.6, \delta_{\mathrm{H}}=1.32,1.13\right)$ by ${ }^{1} \mathrm{H},{ }^{1} \mathrm{H}$ COSY and ${ }^{1} \mathrm{H},{ }^{13} \mathrm{C}$ HMBC correlations completed the structure of this part as 3-amino-14methyl-hexadecanoic acid ( $\beta-A a)$. The sequence of the amino 


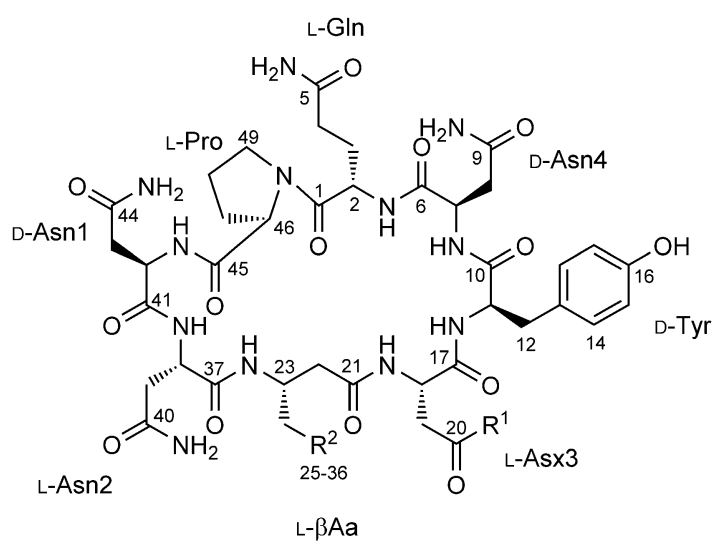

Paenilarvin A (1): $\mathrm{R}^{1}=\mathrm{NH}_{2}, \mathrm{R}^{2}=\left(\mathrm{CH}_{2}\right)_{9} \mathrm{CHCH}_{3} \mathrm{CH}_{2} \mathrm{CH}_{3}$ Paenilarvin B (2): $\mathrm{R}^{1}=\mathrm{OH}, \quad \mathrm{R}^{2}=\left(\mathrm{CH}_{2}\right)_{9} \mathrm{CHCH}_{3} \mathrm{CH}_{2} \mathrm{CH}_{3}$ Paenilarvin $\mathrm{C}(\mathbf{3}): \mathrm{R}^{1}=\mathrm{NH}_{2}, \mathrm{R}^{2}=\left(\mathrm{CH}_{2}\right)_{8} \mathrm{CH}\left(\mathrm{CH}_{3}\right)_{2}$

Scheme 1. Formulas of paenilarvins.

acid residues in $\mathbf{1}$ was established by analysis of HMBC and ROESY correlations, thereby showing connections of the $\alpha$-me- thine protons of amino acid residues to carbonyl carbons of the neighboring residues (Figure 1 and Table 1).

\section{MS analysis}

The sequence of the amino acid residues was analyzed by ESI TOF MSMS and on Orbitrap FTMS mass spectrometer analyzer. The full scan spectrum of 1 showed singly and doubly charged molecular ions at $\mathrm{m} / \mathrm{z} 1112.6108[\mathrm{M}+\mathrm{H}]^{+}$and $\mathrm{m} / \mathrm{z} 556.8098$ $[M+2 \mathrm{H}]^{2+}$ (Figure S12 in the Supporting Information); the MS1 spectrum of paenilarvin B (2) showed similar singly and doubly charged ions at $\mathrm{m} / \mathrm{z} 1113.5963[\mathrm{M}+\mathrm{H}]^{+}$and $\mathrm{m} / \mathrm{z} 557.3026$ $[M+2 \mathrm{H}]^{2+}$ (Figure S25), and the MS1 spectrum of paenilarvin $C$ (3) showed values at $\mathrm{m} / \mathrm{z} 1084.5804[\mathrm{M}+\mathrm{H}]^{+}$and $\mathrm{m} / \mathrm{z} 542.7948$ $[M+2 \mathrm{H}]^{2+}$ (Figure S39). In the MS2 spectra of 1 , based on $\mathrm{m} / \mathrm{z}$ $[M+H]^{+} 1112.610$, two fragments were observed $(\mathrm{m} / \mathrm{z} 707.44$ and 406.17). These two fragments are consistent with the partial amino acid sequences Pro-Asn-Asn- $\beta$-Aa-Asn and Gln-AsnTyr, both in good agreement with the HMBC- and ROESY-NMR data. Compounds $\mathbf{1}$ and $\mathbf{3}$ showed the same amino acid sequence (Gln-Asn-Tyr-Asn- $\beta$-Aa-Asn-Asn-Pro) but with different $\beta$-amino-acid side chains. MS3 spectra showed immonium ions for the side chains $\left(\mathrm{NH}_{2}{ }^{+}=\mathrm{CH}-\right.$ $\left.\mathrm{CH}_{14} \mathrm{H}_{29}\right)$ at $m / z 226.167$ in 1 and Table 1. ${ }^{1} \mathrm{H}\left(700 \mathrm{MH}_{\mathrm{Z}}\right)$ and ${ }^{13} \mathrm{C}(175 \mathrm{MHz}) \mathrm{NMR}$ data of 1 in $\mathrm{CD}_{3} \mathrm{OH}$.

\begin{tabular}{|c|c|c|c|c|c|c|}
\hline Amino acid & Position & $\delta_{c}$ & $\delta_{\mathrm{H}}(J[\mathrm{~Hz}])$ & $\cos Y$ & $\mathrm{HMBC}$ & $\begin{array}{l}\text { ROESY selected } \\
\text { signals }\end{array}$ \\
\hline \multirow[t]{9}{*}{ Gln } & 1 & 172.8 & & & $2,2 \mathrm{NH}, 46$ & \\
\hline & 2 & 51.7 & $4.65(\mathrm{~m})$ & $3 a b, \mathrm{NH}$ & $3 a b, 4 a b$ & $49 a b$ \\
\hline & $3 a$ & 27.9 & $2.11(\mathrm{~m})$ & $2,3 b, 4 a b$ & $2,4 a b$ & $2,49 b ; 2,4 a$ \\
\hline & $3 b$ & & $2.06(\mathrm{~m})$ & $2,3 a, 4 a b$ & & $2,4 a$ \\
\hline & $4 a$ & 31.9 & $2.33(\mathrm{~m})$ & $3 a b$ & $2,5 \mathrm{NH}_{2}$ & \\
\hline & $4 b$ & & $2.32(\mathrm{~m})$ & $3 a b$ & & \\
\hline & 5 & 177.8 & & & $3,4 \mathrm{ab}, 5 \mathrm{NH}$ & \\
\hline & $2 \mathrm{NH}$ & & $7.46(d, 8.0)$ & 2 & & $2,3 a b$ \\
\hline & $5 \mathrm{NH}_{2}$ & & $7.53(\mathrm{~s}), 6.81(\mathrm{~s})$ & & & $3 a b, 4 a b$ \\
\hline \multirow[t]{7}{*}{ Asn4 } & 6 & 173.1 & & & $2,2 \mathrm{NH}, 7,8 \mathrm{ab}$ & \\
\hline & 7 & 52.5 & $4.64(\mathrm{~m})$ & $7 \mathrm{NH}, 8 \mathrm{ab}$ & $8 a b$ & \\
\hline & $8 a$ & 36.9 & $2.73(\mathrm{~m})$ & $7,8 b$ & 7 & 7 \\
\hline & $8 b$ & & $2.70(\mathrm{~m})$ & $7,8 a$ & & 7 \\
\hline & 9 & 175.0 & & & $7,8 \mathrm{ab}, 9 \mathrm{NH} 2$ & \\
\hline & $7 \mathrm{NH}$ & & $8.28(d, 6.7)$ & 7 & 11 & $8 a b, 11$ \\
\hline & $9 \mathrm{NH} 2$ & & $7.54(\mathrm{~s}), 6.85(\mathrm{~s})$ & & & $8 a b$ \\
\hline \multirow[t]{9}{*}{ Tyr } & 10 & 174.3 & & & $7,11 \mathrm{NH}, 12 \mathrm{ab}$ & \\
\hline & 11 & 58.4 & $4.28(\mathrm{~m})$ & $11 \mathrm{NH}, 12 \mathrm{ab}$ & $12 \mathrm{ab}$ & $12 a b, 14$ \\
\hline & $12 a$ & 36.8 & $3.09(\mathrm{dd}, 14.4,5)$ & $11,12 b$ & 11,14 & $11,12 b, 14$ \\
\hline & $12 b$ & & $2.91(\mathrm{~m})$ & $11,12 \mathrm{a}$ & & \\
\hline & 13 & 128.6 & & & $11,12 a b, 15$ & \\
\hline & $14^{*}$ & 131.3 & $7.06(d, 8.4)$ & 15 & $12 \mathrm{ab}$ & $11,12 a b$ \\
\hline & $15^{*}$ & 116.5 & $6.72(d, 8.4)$ & 14 & & \\
\hline & 16 & 157.4 & & & 14,15 & \\
\hline & $11 \mathrm{NH}$ & & $8.49(\mathrm{~d}, 8.0)$ & 11 & & $12 a b, 18$ \\
\hline \multirow[t]{7}{*}{ Asn3 } & 17 & 175.0 & & & $\begin{array}{l}11,11 \mathrm{NH}, 18 \\
19\end{array}$ & \\
\hline & 18 & 52.7 & $4.61(\mathrm{dd}, 13.5,7)$ & $18 \mathrm{NH}, 19 \mathrm{ab}$ & $19 a b$ & \\
\hline & $19 a$ & 38.2 & $\begin{array}{l}2.56(\mathrm{dd}, 15.3 \\
7.5)\end{array}$ & $18,19 b$ & $18,20 \mathrm{NH}_{2}$ & 18 \\
\hline & $19 \mathrm{~b}$ & & $2.48(\mathrm{~m})$ & $18,19 a$ & & $18,23,24 b$ \\
\hline & 20 & 174.6 & & & $18,19,20 \mathrm{NH}_{2}$ & \\
\hline & $18 \mathrm{NH}$ & & $8.01(d, 4.7)$ & 18 & & $22 a b, 23$ \\
\hline & $20 \mathrm{NH}_{2}$ & & $7.58(\mathrm{~s}) 6.81(\mathrm{~s})$ & & & $19 a b$ \\
\hline $\begin{array}{l}\beta \text {-amino- } \\
\text { acid }\end{array}$ & 21 & 174.2 & & & $18 \mathrm{NH}$ & \\
\hline
\end{tabular}
2, and $\left(\mathrm{NH}_{2}{ }^{+}=\mathrm{CH}-\mathrm{C}_{12} \mathrm{H}_{25}\right)$ at $\mathrm{m} / \mathrm{z}$ 198.220 in 3.

The stereochemistry of 1 was determined by Marfey's method. ${ }^{[20]}$ After final hydrolysis, the solution was compared with standard L- and D-amino acids and authentic amino acid derivatives by co-injection. Thus, 1 was found to contain one L-Pro, two L-Asn, two D-Asn, one L-Gln, and one D-Tyr. The positions of the $D-$ and L-Asn residues were assigned by analysis of the $2 \mathrm{D}$ ROESY spectrum analogously to the cyclic lipopeptides maribasins $A$ and $B$, which similarly comprise seven amino acid residues and the same type of side chain. ${ }^{[21]}$ Accordingly, the stereochemistry was deduced by mapping the 2D ROESY correlations between the $\alpha$-proton and the amide proton of the first amino acid (AS1) and between the amide proton of AS1 and the $\alpha$ proton of AS2 (both have the same configuration). The strong ROESY correlation from the Asn 1 amide proton $\left(\delta_{\mathrm{H}}=8.55\right)$ with the L-Pro $\alpha$-proton $\left(\delta_{\mathrm{H}}=4.23\right)$ and the absence of a correlation with the Asn1 $\alpha$-proton $\left(\delta_{H}=\right.$ 


\begin{tabular}{|c|c|c|c|c|c|c|}
\hline Amino acid & Position & $\delta_{\mathrm{C}}$ & $\delta_{\mathrm{H}}(J[\mathrm{~Hz}])$ & COSY & $\mathrm{HMBC}$ & $\begin{array}{l}\text { ROESY selected } \\
\text { signals }\end{array}$ \\
\hline \multirow[t]{12}{*}{$(\beta-\mathrm{Aa})$} & $22 \mathrm{a}$ & 44.2 & $2.49(\mathrm{~m})$ & 23 & $23 \mathrm{NH}, 24 \mathrm{a}$ & $23 \mathrm{NH}, 24 \mathrm{a}$ \\
\hline & $22 b$ & & $2.41(\mathrm{~d}, 14.0)$ & 23 & & $23,24 b$ \\
\hline & 23 & 48.5 & $4.15(\mathrm{~m})$ & $\begin{array}{l}22 \mathrm{ab}, 23 \mathrm{NH}, \\
24 \mathrm{ab}\end{array}$ & $21,22 \mathrm{ab}$ & $22 b, 24 b, 25-33$ \\
\hline & $24 a$ & 36.2 & $1.65(\mathrm{~m})$ & $23,25 \mathrm{ab}$ & $22 b$ & $22 \mathrm{a}, 23,23 \mathrm{NH}, 25-33$ \\
\hline & $24 b$ & & $1.50(\mathrm{~m})$ & $23,25 a b$ & & $22 a b, 23$ \\
\hline & $25-33$ & $\begin{array}{l}27.1- \\
31.2\end{array}$ & $1.33-1.09(\mathrm{~m})$ & & & \\
\hline & 34 & 35.7 & $1.29(\mathrm{~m})$ & $34 \mathrm{Me}, 35$ & $34 \mathrm{Me}, 36 \mathrm{Me}$ & \\
\hline & $34 \mathrm{Me}$ & 19.7 & $0.86(d, 6.2)$ & 34 & 33,35 & \\
\hline & $35 a$ & 30.6 & $1.32(\mathrm{~m})$ & $34,36 \mathrm{Me}$ & $34 \mathrm{Me}, 36 \mathrm{Me}$ & \\
\hline & $35 b$ & & $1.13(\mathrm{~m})$ & $34,36 \mathrm{Me}$ & & \\
\hline & $36 \mathrm{Me}$ & 11.8 & $0.87(t, 7.3)$ & $35 a b$ & 34 & \\
\hline & $23 \mathrm{NH}$ & & $7.49(d, 9.5)$ & 23 & & 23,38 \\
\hline \multirow[t]{7}{*}{ Asn2 } & 37 & 173.6 & & & $23 \mathrm{NH}, 39 \mathrm{ab}$ & \\
\hline & 38 & 51.7 & $4.92(\mathrm{~m})$ & $38 \mathrm{NH}, 39 \mathrm{ab}$ & $39 a b$ & \\
\hline & $39 a$ & 38.8 & $\begin{array}{l}2.97(\mathrm{dd}, 15.7 \\
5.1)\end{array}$ & $38,39 b$ & $40 \mathrm{NH}_{2}$ & $40 \mathrm{NH}_{2}$ \\
\hline & $39 b$ & & $\begin{array}{l}2.61(\mathrm{dd}, 15.7 \\
8.6)\end{array}$ & $38,39 a$ & & $39 a$ \\
\hline & 40 & 174.9 & & & $39 \mathrm{ab}, 40 \mathrm{NH}_{2}$ & \\
\hline & $38 \mathrm{NH}$ & & $7.73(d, 7.5)$ & 38 & & $38,39 b$ \\
\hline & $40 \mathrm{NH}_{2}$ & & $7.44(\mathrm{~s}), 6.85(\mathrm{~s})$ & & & $39 a b$ \\
\hline \multirow[t]{7}{*}{ Asn1 } & 41 & 172.9 & & & 38,43 & \\
\hline & 42 & 52.0 & $4.48(\mathrm{~m})$ & $42 \mathrm{NH}, 43 \mathrm{ab}$ & $43 a b$ & \\
\hline & $43 a$ & 36.7 & $2.92(\mathrm{~m})$ & $42,43 b$ & $42,44 \mathrm{NH}_{2}$ & $42,44 \mathrm{NH}_{2}$ \\
\hline & $43 b$ & & $2.84(\mathrm{dd}, 16,4.5)$ & $42,43 a$ & & 42 \\
\hline & 44 & 175.4 & & & $42,44 \mathrm{NH}_{2}$ & \\
\hline & $42 \mathrm{NH}$ & & $8.55(d, 6.9)$ & 42 & & 46,47 \\
\hline & $44 \mathrm{NH}_{2}$ & & $7.61(\mathrm{~s}), 6.85(\mathrm{~s})$ & & & $43 a b$ \\
\hline \multirow[t]{8}{*}{ Pro } & 45 & 175.4 & & & $46,47 a b$ & \\
\hline & 46 & 63.3 & $4.23(\mathrm{dd}, 7.5,7.5)$ & $47 a b$ & $47,48,49 a b$ & $47 a, 48 b$ \\
\hline & $47 a$ & 30.6 & $2.25(\mathrm{~m})$ & $46,48 a b$ & 46 & $46,48 b$ \\
\hline & $47 b$ & & $1.91(\mathrm{~m})$ & $46,48 a b$ & & \\
\hline & $48 a$ & 26.2 & $2.10(\mathrm{~m})$ & $47 a b, 48 b, 49 a b$ & $46,47 a, 49 b$ & $47 a$ \\
\hline & $48 b$ & & $1.96(\mathrm{~m})$ & $47 a b, 48 a, 49 a b$ & & \\
\hline & $49 a$ & 49.1 & $4.01(\mathrm{~m})$ & $48 a b, 49 b$ & $2,47 a, 48 b$ & $2,48 b, 49 b$ \\
\hline & $49 b$ & & $\begin{array}{l}3.75 \text { (ddd, 17, 7, } \\
6)\end{array}$ & $48 a b, 49 a$ & & $2,48 a, 49 a$ \\
\hline
\end{tabular}

4.48) indicated that the amino acid side chain is on the opposite side of the main plane of the molecule. Thus, Asn1 was assigned the $\mathrm{D}$ configuration. The Asn2 amide proton $\left(\delta_{H}=7.73\right)$ showed correlation with neither the Asn $_{1} \alpha$-proton $\left(\delta_{H}=4.48\right)$ nor the Asn2 $\alpha$-proton $\left(\delta_{\mathrm{H}}=4.92\right)$, thus implying an L configuration for Asn2. ROESY correlations of the amide protons and $\alpha$-protons of Asn2, $\beta$-proton of $\beta-A a$ ( $\mathrm{A} a=$ amino $\quad$ acid)and Asn3 showed that they were on the same side. Thus, $\beta$-Aa and Asn3 have $L$ configurations (Scheme 1). Corresponding to the $\mathrm{D}$ configuration of Tyr derived by Marfey's method, a ROESY correlation of the Tyr amide proton $\left(\delta_{\mathrm{H}}=8.49\right)$ with the Asn3 $\alpha$-proton $\left(\delta_{\mathrm{H}}=4.61\right)$ was found, but no correlation with the Tyr $\alpha$-proton $\left(\delta_{\mathrm{H}}=4.28\right)$. The ROESY correlation of the amide proton $\left(\delta_{H}=8.28\right)$ of Asn4 with the $\alpha$-proton $\left(\delta_{\mathrm{H}}=4.28\right)$ of Tyr and with the $\alpha$-proton $\left(\delta_{\mathrm{H}}=\right.$ 4.64) of Asn4 suggested a D configuration for Asn4. In contrast, no correlation was observed for amide proton $\left(\delta_{\mathrm{H}}=7.46\right)$ of L-Gln with the $\alpha$-proton of Asn4. All correlations are in complete accordance with the mojavensin structure described by Ma et al. ${ }^{[15]}$ The structure of $\mathbf{1}$ was finally established as cyclo L-ProD-Asn1-L-Asn2-L- $\beta$-amino-14-methylhexadecanoic acid-L-Asn3D-Tyr-D-Asn4-L-Gln (belonging to the iturin family).

In addition to paenilarvins A, B, and C, four further paenilarvin variants were identified in low concentrations in the crude extracts. Masses of 1097.6 and 1069.7 Da for two of these suggested the subsequent removal of a $\mathrm{CH}_{2}$ group $\left(M_{\mathrm{W}}=14\right)$ from the $\beta$-amino side chain of paenilarvin $A$ and paenilarvin $C$, respectively. Another two variants (1084.6 Da) seem to be variants of paenilarvin B with changes in chain length and stereochemistry in the $\beta$-amino side chain. However, the absolute configurations of the side chains remain to be solved for all paenilarvins.

Iturin family compounds produced by Bacillus spp. have been characterized as lipopeptides with a $\beta$-amino fatty acid chain linked to a circular heptapeptide. ${ }^{[22]}$ The first report of an iturin-like compound from a Paenibacillus species came in 2000: from a chitinolytic bacterial strain, Paenibacillus koreensis $\mathrm{YC} 00^{\top}$, isolated from a compost sample (Chinju, Republic of 
Korea), but the complete molecular structure of the compound was not described. ${ }^{[23]}$

\section{In silico analysis of the paenilarvin biosynthetic gene cluster}

The potential production by $P$. larvae of bioactive secondary metabolites was confirmed following complete genome sequencing of P.larvae DSM 25719 (ERIC I) and DSM 25430 (ERIC II), recently reported by Djukic et al. (2014). ${ }^{[14]}$ Four different NRPS or NRPS/PKS hybrid clusters with variations in domain organization and size of clusters between the two genotypes were identified during this study, based on genome analysis and comparison.

Here, we focus on an NRPS-(trans-AT)-PKS hybrid cluster ( $37 \mathrm{~kb}, \mathrm{GC}$ content $43.7 \%$ ). This showed high similarity to the clusters for iturin $\mathrm{A}^{[24]}$ and mycosubtilin ${ }^{[25]}$ biosynthesis in Bacillus subtilis (Figure S41), and high sequence identity with NRPS/ PKS hybrid gene clusters identified in Bacillus atrophaeus and Bacillus amyloliquefaciens. The predicted sequence of the putative monomeric lipopeptide was Mal-(Pk-Asn)-Tyr-Asn-Gln-ProAsn-Ser, which correlates with the amino acid composition of iturin A. A model for the biosynthesis of iturin family lipopeptides has been described by characterizing various domains of mycosubtilin synthetase. ${ }^{[25]}$ This model can be employed to describe paenilarvin biosynthesis, based on gene-sequence and structural similarity to iturin compounds.

Detailed sequence analysis of the cluster revealed the presence of four ORFs, all transcribed in the same direction (Figure 2). ORF ERIC2_c18780 encodes an acyl transferase domain; the downstream ORFs ERIC2_c18770-ERIC2_c18750 encode NRPS or NRPS/PKS domains (Table 2). Of seven amino acid-activating modules identified in the cluster, one is in ERIC2_c18770, four are in ERIC2_c18760, and two are in ERIC2_ c18750 (Figure 2). Epimerization domains were identified in modules two, three and six (Figure 2), thus indicating conversion of the activated amino acids Tyr, Asn4, and Asn1 into the D-configuration, consistent with the conclusions from the Marfey's and ROESY analysis (Scheme 1). Finally, ERIC2_c18750 contains the thioesterase domain, which is supposedly required for release and putative cyclization of the lipopeptide molecule. A complete putative paenilarvin gene cluster is present only in P. larvae DSM 25430 (ERIC II), thus suggesting that P. larvae DSM 25719 (ERIC I) is unable to produce the corresponding lipopeptide (Scheme 1). However, DSM 25719 harbors a NRPS possibly involved in the production of a smaller peptide.

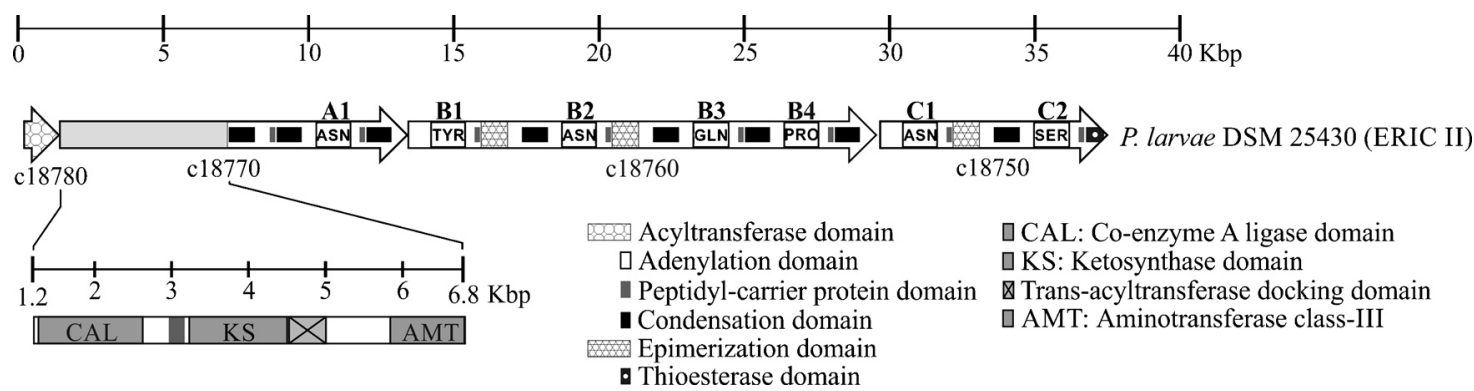

Figure 2. Genetic organization and domain structure of the putative paenilarvin gene cluster, comprising the ORFs c18780, c18770, $\mathrm{c} 18760$ and $\mathrm{c} 18750$. Seven modules (A1, B1-B4, C1, C2) are depicted. The deduced domain organizations of the different proteins specified by the gene cluster are indicated (antiSMASH domain prediction into translated proteins).

Table 2. Deduced functions of the ORFs identified within the paenilarvin gene cluster based on percentage sequence identity and coverage to previously known proteins as well as to the Bacillus subtilis RB14 iturin A biosynthetic gene cluster.

\begin{tabular}{|c|c|c|c|c|c|}
\hline Gene & Residues & Proposed function ${ }^{[a]}$ & $\begin{array}{l}\text { Closest similar protein, organism, } \\
\text { accession no. }(\text { size })^{[b]}\end{array}$ & $\begin{array}{l}\text { Sequence } \\
\text { identity/ } \\
\text { query } \\
\text { coverage [\%] }\end{array}$ & 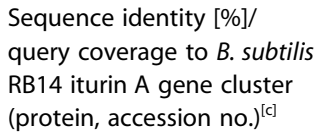 \\
\hline $\begin{array}{l}\text { ERIC2 } \\
\text { c18780 }\end{array}$ & 399 & $\begin{array}{l}\text { malonyl CoA-acyl carrier protein } \\
\text { transacylase }\end{array}$ & $\begin{array}{l}\text { malonyl CoA: acyl carrier protein transacylase-like } \\
\text { protein, Paenibacillus larvae, WP_023484391 (399) }\end{array}$ & $99 / 100$ & 63/99 (ItuD, BAB69697) \\
\hline $\begin{array}{l}\text { ERIC2 } \\
\text { c18770 }\end{array}$ & 3984 & $\begin{array}{l}\text { NRPS/PKS (CAL-domain, PCP, KS, } \\
\text { trans-AT, aminotransferase type III, } \\
C, P C P, C, A, P C P, C)\end{array}$ & $\begin{array}{l}\text { surfactin synthetase-like protein, Paenibacillus } \\
\text { larvae, WP_023484390 (3767) }\end{array}$ & $97 / 99$ & 68/100 (ItuA, BAB69698) \\
\hline $\begin{array}{l}\text { ERIC2 } \\
\text { C18760 }\end{array}$ & 5369 & $\begin{array}{l}\text { NRPS (A, PCP, E, C, A, PCP, E, C, A, } \\
P C P, C, A, P C P, C)\end{array}$ & $\begin{array}{l}\text { mycosubtilin synthase subunit B, Bacillus } \\
\text { atrophaeus, WP_010788882 (5363) }\end{array}$ & $72 / 100$ & 70/100 (ItuB, BAB69699) \\
\hline $\begin{array}{l}\text { ERIC2 } \\
\text { C18750 }\end{array}$ & 2601 & NRPS (A, PCP, E, C, A, PCP, TE) & $\begin{array}{l}\text { iturin A synthetase C, Bacillus amyloliquefaciens } \\
\text { subsp. plantarum UCMB5036, YP_007497454 (2617) }\end{array}$ & $65 / 99$ & 65/99 (ItuC, BAB69700) \\
\hline
\end{tabular}

[a] Domain organization as proposed by antiSMASH (Blin et al. 2013): PCP: peptidyl carrier protein, KS: ketosynthase domain, C: condensation domain, AT: acyl transferase, A: adenylation domain, E: epimerization domain, TE: thioesterase domain. [b] Protein Blast (Database: nonredundant protein sequences $(\mathrm{nr}))$; size $=$ number of amino acid residues. [C] NCBI Align Sequences Protein BLAST (BLOSUM62, Gap open penalty: 11, Gap extension penalty: 1). 


\section{Antimicrobial and cytotoxic activities of paenilarvins}

Although 1 and 2 showed no activity against the Gram-positive and Gram-negative bacteria tested, most of the fungi and yeasts were inhibited by both the lipopeptides. Compared to 2, 1 showed much better antifungal activity $\left(\mathrm{MIC}=2.1 \mu \mathrm{g} \mathrm{mL}^{-1}\right.$ against Trichosporon oleaginous, Aspergillus clavatus, Botryotinia fuckeliana, and Hormoconis resinae; Table 3). Compound 2 was

\begin{tabular}{|c|c|c|c|c|}
\hline & Test organism & Paenilarvin A & Paenilarvin B & Control \\
\hline \multirow[t]{8}{*}{ Yeasts } & Saccharomyces cerevisiae (DSM 70449) & 4.2 & 33.3 & $4.2^{[b]}$ \\
\hline & Rhodotorula glutinis (DSM 10134) & 4.2 & 16.6 & $<0.52^{[b]}$ \\
\hline & Candida albicans (DSM 1665) & 4.2 & n.i. & $8.3^{[c]}$ \\
\hline & Wickerhamomyces anomalus (DSM 6766) & 4.2 & 16.6 & $2.1^{[b]}$ \\
\hline & Nematospora coryli (DSM 6981) & 4.2 & 33.3 & $3.3^{[b]}$ \\
\hline & Trichosporon oleaginous (DSM 11815) & 2.1 & 16.6 & $0.52^{[b]}$ \\
\hline & Debaryomyces hansenii (DSM 3428) & 4.2 & n.i. & $8.3^{[b]}$ \\
\hline & Pichia membranifaciens (DSM 21959) & 4.2 & 33.3 & $0.052^{[b]}$ \\
\hline \multirow[t]{5}{*}{ Filamentous fungi } & Mucor hiemalis (DSM 2656) & 4.2 & 33.3 & $2.1^{[b]}$ \\
\hline & Aspergillus clavatus (DSM 816) & 2.1 & 8.3 & $2.1^{[b]}$ \\
\hline & Botryotinia fuckeliana (DSM 877) & 2.1 & 16.6 & $2.1^{[b]}$ \\
\hline & Hormoconis resinae (DSM 1203) & 2.1 & 16.6 & $<0.25^{[b]}$ \\
\hline & Penicillium capsulatum (DSM 2210) & 4.2 & 33.3 & $16.6^{[\mathrm{b}]}$ \\
\hline \multirow[t]{5}{*}{ Gram positive bacteria } & Nocardioides simplex (DSM 20130) & n.i. & n.i. & $16.6^{[\mathrm{a}]}$ \\
\hline & Nocardia sp. (DSM 43069) & n.i. & n.i. & $<0.52^{[\mathrm{a]}]}$ \\
\hline & Staphylococcus aureus (DSM 346) & n.i. & n.i. & $0.1^{[\mathrm{a}]}$ \\
\hline & Micrococcus luteus (DSM 20030) & n.i. & n.i. & $3.3^{[\mathrm{a}]}$ \\
\hline & Paenibacillus polymyxa (DSM 36) & n.i. & n.i. & $6.7^{[\mathrm{a}]}$ \\
\hline \multirow[t]{2}{*}{ Gram negative bacteria } & Pseudomonas aeruginosa (DSM 50071) & n.i. & n.i. & $0.13^{[\mathrm{d}]}$ \\
\hline & Chromobacterium violaceum (DSM 30191) & n.i. & n.i. & $1.0^{[\mathrm{a}]}$ \\
\hline
\end{tabular}

moderately active against fungi and yeast (lowest MIC= $16.6 \mu \mathrm{g} \mathrm{mL}^{-1}$ ). When 1 and $\mathbf{2}$ were tested for their cytotoxicity against mouse fibroblast cell line L929, 1 was again found to be more active $\left(I C_{50}=4 \mu \mathrm{gL}^{-1}\right)$ than $2\left(\mathrm{IC}_{50}>10 \mu \mathrm{g} \mathrm{mL}^{-1}\right)$.

Lipopeptides of the iturin family (iturin, mycosubtilin, bacillomycin, and bacillopeptin) have long been known to have strong antifungal and hemolytic activity by destroying the cytoplasmic membrane, ${ }^{[26]}$ but show only limited antibacterial effects. $^{[22]}$ The strong antifungal activities of iturin-producing organisms make them promising candidates as biocontrol agents. Mojavensin A was also found to be active against soilborne phytopathogens like Fusarium oxysporum f. sp. cucumerinum and Valsa mali and weakly inhibitory against Staphylococcus aureus (Ma et al., 2012). ${ }^{[15]}$ However, anteiso-C15 mojaven$\sin A$ was not as active against fungi as were the other iturins. Hence, the strong antifungal activity and absence of antibacterial activity of $\mathbf{1}$ and $\mathbf{2}$ against our test organisms correspond to the biological activity data available for other iturins.

\section{Paenilarvin toxicity to bee larvae}

To assess the toxicity of paenilarvins with larvae, $\mathbf{1}$ and $\mathbf{2}$ were administered to bee larvae along with food on day 4 . The assay was continued for 22 days, and the numbers of dead individuals were counted in the control and experimental groups for statistical analysis. The increase in number of killed individuals in toxicity assays was assessed by non-linear regression by using a model for significance test [Eq. (1)]:[27]

$$
f(t)=\frac{N_{0} K \times e^{r t}}{K+N_{0} \times e^{r t-1}}
$$

where $N_{0}$ is the number of dead larvae on day $1, K$ is maximum mortality; $r$ is the expansion rate, and $t$ is time.

Maximum mortalities were derived from nonlinear regression $(t=22)$ and compared with the controls. The mortality at the end of the assay of larvae was $46 \%$ for 1 and $49 \%$ for 2 . These values are $25 \%$ (1) and $35 \%$ (2) higher compared to the control, thus suggesting toxicity of paenilarvins on larval development (Figure $\mathrm{S} 40 \mathrm{~B}$ ). The significance of these results was tested by ANOVA (analysis of variances). No significant differences in mortality were found between 1 and 2, but both differed considerably from the control (Figure S40 C).

These results are preliminary data from the toxicity assays, and further detailed investigations with different concentration of paenilarvins and different larval stages are required to confirm their toxicity on bee larvae.

\section{Conclusion}

$P$. larvae is a pathogen of great economic and environmental concern because of its ability to cause one of the most serious and contagious diseases of honey bees, AFB. We explored the secondary metabolite potential of $P$. larvae after the identification of genome segments homologous to NRPS and PKS clusters in closely related organisms, like $B$. subtilis. ${ }^{[9,10,14]}$

We were able to identify a group of antifungal compounds in the crude extracts of P. larvae DSM 25430 (ERIC II). Purification and molecular characterization of the compounds by mass and NMR spectroscopy revealed that the compounds belong to the iturin family. Complete structure elucidation of the purified compounds revealed two major cyclic compounds: paenilarvins $A$ and $B$. Paenilarvin $A$ showed the same amino acid sequence as mojavensin $A^{[15]}$ but differed in the length of $\beta$-amino acid side chain. Paenilarvin $B$ had the same side chain as paenilarvin $A$ but contained an aspartic acid instead of asparagine at position 2.

Paenilarvins $A$ and $B$ showed no antibacterial activity but strong antifungal activity against rare human pathogenic spe- 
cies like A. clavatus and Penicillium capsulatum and phytopathogens like Nematospora coryli and B. fuckeliana. Paenilarvins also exhibited significant activity against a commercially important fuel-contaminant fungus, $H$. resinae. ${ }^{[28]}$ Cytotoxic activity against mouse fibroblast cell line L929 was also observed. Paenilarvins $A$ and $B$ also showed significant toxicity against honey bee larvae in our preliminary exposure assay.

The production of secondary metabolites usually serves as a survival measure for the producing organisms in their ecological niches. ${ }^{[29]}$ Given that honey bees are exposed to many pathogens, such as parasitic mites, small hive beetle, microsporidian parasites, viruses, and fungi, ${ }^{\left[{ }^{30]}\right.}$ it can be speculated that $P$. larvae produces paenilarvins to gain an advantage over these potential pathogens. Certain other microbes also show entomopathogenic activity: infecting and killing insects with secondary metabolites. ${ }^{[31]}$ However, these hypotheses can only be verified by comprehensively examining the inhibitory effects of these compounds against prospective honey bee pathogens and honey bee larvae.

It is known that antibiotics can exert diverse effects at subinhibitory concentrations, and that the response depends upon the concentration used ("hormensis"). ${ }^{[32]}$ The analysis of effects of subinhibitory antibiotic concentrations has revealed the regulation of key biological processes, including transcription, translation, transport of exoproteins, stress response, quorum sensing, and biofilm formation. ${ }^{[11]}$ It has also been studied in regard to the virulence and pathogenic properties of a number of bacteria. ${ }^{[33]}$ In 2011, aureusimines A and B were reported to be involved in regulation of expression of virulence factors, exotoxins, $\gamma$ hemolysin, and regulatory redox-associated superantigen-like genes in S. aureus. ${ }^{[3]}$ Paenilarvins A and B might also be involved in the regulation of physiological processes in $P$. larvae; this needs to be studied at the molecular level.

Recently, it was determined that P. larvae ERIC I strains lack the $S$ layer protein, and that ERIC II strains do not express PIX1 and PIx2 toxin genes, both of which have been found to be virulence factors for their respective strains..$^{[7,8,35]}$ Hence, these strains use different virulence factors during pathogenesis. If paenilarvins are also found to be key factors in the pathogenesis of $P$. larvae ERIC II strains, this would support the hypothesis that these strains have developed different strategies for infection and disease progression. Thus, our findings might explain the observed differences in virulence between the two strains.

In conclusion, we were able to purify and characterize a group of secondary metabolites produced by P. larvae DSM 25430 (ERIC II); these possibly play an important role for the pathogen. However, their precise function in pathogenesis still needs to be determined. The presence of other biosynthetic clusters in both ERIC I and ERIC II is evident from the genomic data obtained for the pathogen, thus suggesting that other important secondary metabolites remain to be discovered..$^{[9,14]}$ Further studies are needed to unravel the products of these biosynthetic clusters and to investigate their exact roles in pathogenesis. A comprehensive understanding of P. larvae pathogenesis combined with molecular evidence on virulence factors would pave the way for effectively dealing with this devastating honey bee disease.

\section{Experimental Section}

General experimental procedures: Spectral and physico-chemical data were obtained with the following instruments. NMR spectra were recorded on an Ascend $700 \mathrm{TCl}$ cryoprobe spectrometer ( ${ }^{1} \mathrm{H}$ $700 \mathrm{MHZ},{ }^{13} \mathrm{C} 176 \mathrm{MHz}$; Bruker) with $\mathrm{CD}_{3} \mathrm{OH}$ as the solvent. All 2D NMR spectra were analyzed with ACD/Spectrus NMR software (Advanced Chemistry Development, Toronto, Canada). IR spectra were recorded on an FTIR 100 spectrometer (PerkinElmer). UV spectra were measured on a UV/Vis-2102 spectrometer (Shimadzu) with methanol as the solvent. Optical rotation was measured on a model 241 MC spectrometer (PerkinElmer) with methanol or DMSO as the solvent. Mass spectra were obtained on a maXis UHRTOF spectrometer (Bruker) with electrospray ionization (ESI) in positive mode; molecular formulae were calculated, including the isotope pattern (Smart Formula algorithm) and ESI TOF MS-MS spectra from a Finnigan LTQ Orbitrap mass spectrometer (Thermo Scientific). Analytical HPLC: gradient over $25 \mathrm{~min}$; solvent $\mathrm{A}$ : acetonitrile/ water (5:95) with $\mathrm{NH}_{4} \mathrm{CH}_{3} \mathrm{COO}(5 \mathrm{~mm})$, pH $5.5\left(40 \mu \mathrm{L} \mathrm{CH}{ }_{3} \mathrm{COOH} / \mathrm{L}\right)$; solvent $\mathrm{B}$ : acetonitrile/water (95:5) with $\mathrm{NH}_{4} \mathrm{CH}_{3} \mathrm{COO}(5 \mathrm{~mm})$,

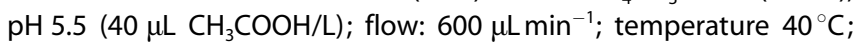
column: Acquity BEH C18 $(50 \times 2.1 \mathrm{~mm}, 1.7 \mu \mathrm{m}$; Waters $)$ equipped with the corresponding pre-column.

Bacterial strains and growth conditions: P. larvae isolates DSM 25719 (genotype ERIC I) and DSM 25430 (ERIC II) were cryopreserved at $-80^{\circ} \mathrm{C}$. Both these strains are available at DSMZ. DSM 25430 was reactivated in MYPGP broth $(20 \mathrm{~mL}, \mathrm{pH} \mathrm{7}$, made in our laboratory) ${ }^{[36]}$ but with beef infusion replaced by meat extract ( $\left.2 \%\right)$ and vitamin solution $(0.2 \%)$. The strain was then subcultured in the same medium $(600 \mathrm{~mL})$ in Erlenmeyer's flasks and used as a seed culture. Scale-up fermentation (5.2 L) of strain DSM 25430 was performed in $1 \mathrm{~L}$ Erlenmeyer flasks in the same medium $(350 \mathrm{~mL})$ supplemented with Amberlite XAD-16 resin (2\%; SigmaAldrich) and incubated at $30^{\circ} \mathrm{C}$ with shaking $(160 \mathrm{rpm})$ for 6 days. $X A D$ resin was separated from the culture at the end of fermentation by sieving. DSM 25719 was grown under the same conditions.

Extraction and isolation of active compounds: Methanol ( $1 \mathrm{~L})$ was used for elution from the adsorber XAD resin packed in a glass column. Methanol was evaporated from the extract to give a dry crude material $(6.14 \mathrm{~g})$. This extract was separated on silica gel $(120 \mathrm{~g})$ in an open column equilibrated with ethyl acetate. Elution from the silica gel was achieved with ethyl acetate/methanol (100\% ethyl acetate to $100 \%$ methanol) to obtain four principal fractions. The organic solvent from each fraction was evaporated.

Fraction 3 ( $1.19 \mathrm{~g}$; eluted with ethyl acetate/methanol (50:50, v/v), $2 \mathrm{~L}$ ) was dissolved in methanol and passed through a polymeric reversed-phase Strata-X cartridge $(33 \mu \mathrm{m}, 200 \mathrm{mg}, 6 \mathrm{~mL}$; Phenomenex Inc., Torrence, CA) and concentrated to $6 \mathrm{~mL}$. Processed samples were further purified by four passes of preparative RP-HPLC (Gemini C18 column: $10 \mu \mathrm{m}, 110 \AA, 250 \times 21.2 \mathrm{~mm}$, Phenomenex); solvent $A$ : acetonitrile/ $\mathrm{H}_{2} \mathrm{O}$ (37:63) with formic acid (0.1\%); solvent B: acetonitrile/ $\mathrm{H}_{2} \mathrm{O}(40: 60)$ plus formic acid $(0.1 \%)$; gradient: $0 \% \mathrm{~B}$ for $35 \mathrm{~min}, 100 \% \mathrm{~B}$ from 35 to $80 \mathrm{~min}$; flow rate $20 \mathrm{~mL} \mathrm{~min}^{-1}$; UV detection at $210 \mathrm{~nm}$. The fractions contained paenilarvins $A$ $(6.8 \mathrm{mg}), B(3.9 \mathrm{mg})$, and C (12.8 mg) along with other related peptides.

Paenilarvin A (1) white, solid; UV (methanol) $\lambda_{\max }(\log \varepsilon)=223(4.27$ $\mathrm{sh}), 277 \mathrm{~nm}(3.35) ;[\alpha]_{\mathrm{D}}=+15.4^{\circ} \quad(c=0.039, \mathrm{DMSO}) ; t_{\mathrm{R}}=10.9 \mathrm{~min}$; $v=3383,2927,2855,1668,1541,1517,1384 \mathrm{~cm}^{-1} ;{ }^{1} \mathrm{H}$ and ${ }^{13} \mathrm{C}$ NMR: see Table 1; HR-ESI-MS: $\mathrm{m} / \mathrm{z} \quad 1112.6108 \quad\left[{\left.\mathrm{M}+\mathrm{H}^{+}\right]}^{+}\right.$(calcd for $\mathrm{C}_{52} \mathrm{H}_{82} \mathrm{~N}_{13} \mathrm{O}_{14}$ 1112.6099). 
Paenilarvin B (2) white, solid; UV (methanol) $\lambda_{\max }(\log \varepsilon)=223$ (4.23), $277 \mathrm{~nm}$ (3.35); $[\alpha]_{\mathrm{D}}=+6.4^{\circ} \quad\left(c=0.075\right.$, methanol); $t_{\mathrm{R}}=11.1 \mathrm{~min}$; ${ }^{1} \mathrm{H}$ NMR $\left(\mathrm{CD}_{3} \mathrm{OH}+5 \mu \mathrm{L} \mathrm{HCOOH}, 700 \mathrm{MHz}\right)$ selected signals: for Asp: $\delta_{\mathrm{C}}=173.0 \mathrm{ppm}$ (C37), not observed (C38), 37.6 (C39), $175.4 \mathrm{ppm}$ (C40), $\delta_{\mathrm{H}}=4.87(\mathrm{~m}, \mathrm{H} 38), 3$ and $2.84(\mathrm{~m}, \mathrm{H} 39 \mathrm{a}, \mathrm{b}), 7.78 \mathrm{ppm}(38 \mathrm{NH})$; HR-ESI-MS: $m / z \quad 1113.5953 \quad\left[M+\mathrm{H}^{+}\right]$(calcd for $\mathrm{C}_{52} \mathrm{H}_{81} \mathrm{~N}_{12} \mathrm{O}_{15}$ 1113.5939).

Paenilarvin C (3) white, solid; UV (methanol) $\lambda_{\max }(\log \varepsilon)=223$ (4.80), $277 \mathrm{~nm} \quad(3.35) ; \quad[\alpha]_{\mathrm{D}}=+11.8^{\circ} \quad(c=0.221, \quad \mathrm{DMSO}) ; \quad t_{\mathrm{R}}=9.6 \mathrm{~min}$; ${ }^{1} \mathrm{H}$ NMR ([D $\left.\mathrm{D}_{6}\right] \mathrm{DMSO}, 700 \mathrm{MHz}$ ) selected signals: end of the side chain: $-\mathrm{CH}_{2}\left(\delta_{\mathrm{C}}=38.5, \delta_{\mathrm{H}}=1.24,1.13 \mathrm{ppm}\right),-\mathrm{CH}\left(\delta_{\mathrm{C}} / 27.4, \delta_{\mathrm{H}} /\right.$ $1.49 \mathrm{ppm}, \mathrm{m}),-\left(\mathrm{CH}_{3}\right)_{2}\left(\delta_{\mathrm{C}}=22.5, \delta_{\mathrm{H}}=0.84, \mathrm{~d}, 6.7 \mathrm{ppm}, \mathrm{Hz}\right) ; \mathrm{HR}-\mathrm{ESI}-$ MS: $m / z 1084.5790\left[M+\mathrm{H}^{+}\right]$(calcd for $\mathrm{C}_{50} \mathrm{H}_{78} \mathrm{~N}_{13} \mathrm{O}_{14}$ 1084.5785).

\section{Assignment of absolute stereochemistry of amino acid residues: Marfey's method was used for determining the stereochemistry of amino acid residues in paenilarvins. Lipopeptide $(1.1 \mathrm{mg})$ was hy- drolyzed in $\mathrm{HCl}(6 \mathrm{~N}, 0.5 \mathrm{~mL})$ at $110^{\circ} \mathrm{C}$ overnight. Then, the sample was dried under vacuum. Water $(50 \mu \mathrm{L}), 1$-fluoro-2-4-dinitrophenyl- 5-L-alanine amide (FDAA, Marfey's reagent; $100 \mu \mathrm{L}$ in acetone, $1 \%$, $w / w)$, and sodium hydrogen carbonate $(1 \mathrm{M}, 20 \mu \mathrm{L})$ were added to the hydrolyzed products, and the mixture was heated at $41^{\circ} \mathrm{C}$ for $1 \mathrm{~h} . \mathrm{HCl}(2 \mathrm{M}, 10 \mu \mathrm{L})$ was added to stop the reaction, and the mix- ture was evaporated to dryness. The residue was dissolved in water/DMSO $(1: 1,0.5 \mathrm{~mL})$ and analyzed by reversed-phase chroma- tography (Waters Acquity UPLC BEH C18 column, $2.1 \times 50 \mathrm{~mm}$, $1.7 \mu \mathrm{m}$; gradient: acetonitrile/water $(5: 95)$ with formic acid $(0.1 \%)$ to acetonitrilie/water (40:60) with formic acid (0.1\%) over $30 \mathrm{~min}$; flow rate $0.6 \mathrm{~mL} \mathrm{~min}^{-1}$; UV detection: DAD, MS: amaZon spectrom- eter (Bruker) in ESI mode.}

Sequence analysis: The genomes of P. larvae DSM 25719 (ERIC I) and DSM 25430 (ERIC II) were sequenced by Djukic et al. ${ }^{[14]}$ The IMG/ER (Intergrated Microbial Genomes/Expert Review) system, ${ }^{[37]}$

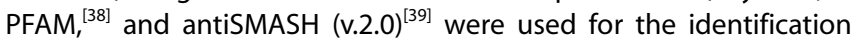
of NRPS/PKS gene clusters in ERIC I and ERIC II genomes (accession numbers: ADFW00000000 and CP003355-CP003356, respectively). The EasyFigure tool (minimum length $250 \mathrm{bp}$, maximum $e$ value 0.001 , minimum identity $65 \%)^{[40]}$ was used for comparison between NRPS/PKS clusters from the two genotypes and identification of similar sequences in other bacteria.

Antimicrobial growth inhibition assay: The MIC values for $\mathbf{1}$ and 2 were determined against a number of bacteria and fungi (Table 3). The serial dilution assay was carried out as previously described ${ }^{[41]}$ The starting concentrations of 1, 2, and reference drugs (oxytetracycline hydrochloride (Sigma-Aldrich) dissolved in water; nystatin dihydrate (Sigma-Aldrich) dissolved in $\mathrm{MeOH}$ or DMSO, and gentamycin (SERVA Electrophoresis, Heidelberg, Germany) dissolved in water) were varied according to the activity.

Cytotoxicity assay: Compounds $\mathbf{1}$ and $\mathbf{2}$ were also tested for their cytotoxic activity against mouse fibroblast cell line L929 cultured in DMEM (Lonza) supplemented with $10 \%$ fetal bovine serum (Gibco). The $\mathrm{IC}_{50}$ value was determined by using an MTT assay as previously described. ${ }^{[41]}$

Paenilarvin toxicity bioassay against larvae: Exposure in vivo bioassays were performed as described previously. ${ }^{[42]}$ Queens of two different healthy A. mellifera subsp. carnica bee colonies (from the apiary of one of the authors) were confined on an empty comb in an excluder cage (day -3$)$ and taken out after $24 \mathrm{~h}$ (day -2 ). Larvae started hatching from the eggs on day +1 and were transferred onto diet A ( $20 \mu \mathrm{L}, 50 \%$ royal jelly, $50 \%$ aqueous solution containing yeast extract (2\%), fructose $(12 \%)$ and glucose $(12 \%)$ within $24 \mathrm{~h}$ of egg hatching (day $1, \mathrm{~d} 1$ ). On day $3(\mathrm{~d} 3)$, the larvae were fed diet $B(20 \mu \mathrm{L}, 50 \%$ royal jelly, $50 \%$ aqueous solution containing yeast extract $(3 \%)$, fructose $(15 \%)$ and glucose $(15 \%))$ followed by diet C (50\% royal jelly, $50 \%$ aqueous solution containing yeast extract $(4 \%)$, fructose $(18 \%)$ and glucose $(18 \%))$ from day 4 to day $6(30,40$, and $50 \mu \mathrm{L}$ on successive days). Food was not needed for later metamorphosis. To test the bioactivity of paenilarvins on developing $A$. mellifera, a larval group was fed with diet $C$ supplemented with paenilarvins (A or $\mathrm{B} ; 5 \mu \mathrm{g}$ in $\mathrm{ddH}_{2} \mathrm{O}$ ) on day 4 (control larvae fed normally). Larvae were inspected between days 4 and 22 (but not on days 6/7, 13/14, 20/21) and categorized as dead when they stopped breathing or moving. Dead individuals were removed from the well plate assay.

\section{Acknowledgements}

S.S. is highly indebted to Erasmus Mundus External Cooperation Window for a PhD scholarship and their support. H.B. was supported by the MINAS graduate school. We acknowledge Christel Kakoschke, Silke Reineke, Aileen Teichmann, Diana Telkemeyer, and Wera Collisi for their technical assistance. We are grateful to Dr. Alberto Plaza for proofreading this manuscript.

Keywords: American foulbrood - antifungal agents .
lipoproteins - Paenibacillus larvae - paenilarvins - secondary
metabolites

[1] E. Genersch, Invertebr. Pathol. 2010, 103, S10-S19.

[2] E. Genersch, E. Forsgren, J. Pentikäinen, A. Ashiralieva, S. Rauch, J. Kilwinski, I. Fries, Int. J. Syst. Evol. Microbiol. 2006, 56, 501 - 511.

[3] a) E. Genersch, A. Ashiralieva, I. Fries, Appl. Environ. Microbiol. 2005, 71, $7551-7555$; b) S. Rauch, A. Ashiralieva, K. Hedtke, E. Genersch, Appl. Environ. Microbiol. 2009, 75, 3344-3347.

[4] D. Yue, M. Nordhoff, L. H. Wieler, E. Genersch, Environ. Microbiol. 2008, 10, $1612-1620$.

[5] a) K. Antúnez, M. Anido, J. D. Evans, P. Zunino, Vet. Microbiol. 2010, 141, 385-389; b) K. Antúnez, M. Anido, D. Arredondo, J. D. Evans, P. Zunino, Vet. Microbiol. 2011, 147, 83-89.

[6] K. Antúnez, D. Arredondo, M. Anido, P. Zunino, Microbiol. 2011, 157, $1474-1480$.

[7] L. Poppinga, B. Janesch, A. Fünfhaus, G. Sekot, E. Garcia-Gonzalez, G. Hertlein, K. Hedtke, C. Schäffer, E. Genersch, PLoS Pathog. 2012, 8, e1002716.

[8] A. Fünfhaus, L. Poppinga, E. Genersch, Environ. Microbiol. 2013, 15, $2951-2965$.

[9] A. Fünfhaus, A. Ashiralieva, R. Borriss, E. Genersch, Environ. Microbiol. Rep. 2009, 1, 240-250.

[10] Q. W. T. Chan, R. S. Cornman, I. Birol, N. Y. Liao, S. K. Chan, T. R. Docking, S. D. Jackman, G. A. Taylor, S. J. M. Jones, D. C. de Graaf, J. D. Evans, L. J. Foster, BMC Genomics 2011, 12, 450.

[11] a) A. L. Demain, J. Ind. Microbiol. Biotechnol. 2006, 33, 486-495; b) D. Romero, M. F. Traxler, D. López, R. Kolter, Chem. Rev. 2011, 111, 5492 5505.

[12] H.-A. Schild, S. W. Fuchs, H. B. Bode, B. Grünewald, Appl. Environ. Microbiol. 2014, 80, 2484-2492.

[13] E. Garcia-Gonzalez, S. Müller, P. Ensle, R. D. Süssmuth, E. Genersch, Environ. Microbiol. 2014, 16, 1297-1309.

[14] M. Djukic, E. Brzuszkiewicz, A. Fünfhaus, J. Voss, K. Gollnow, L. Poppinga, H. Liesegang, E. Garcia-Gonzalez, E. Genersch, R. Daniel, PLoS One 2014, 9, e90914.

[15] Z. Ma, N. Wang, J. Hu, S. Wang, J. Antibiot. 2012, 65, 317-322.

[16] Z. Gliński, J. Jarosz, Apidologie 1992, 23, 193-201.

[17] a) . L.-j. Zhao, X.-n. Yang, X.-y. Li, W. Mu, F. Liu, Agric. Sci. China 2011, 10, 728-736; b) W. Raza, X. Yang, H. Wu, Y. Wang, Y. Xu, Q. Shen, Eur. J. Plant Pathol. 2009, 125, 471-483; c) P. G. Stansly, R. G. Shepard, H. J. 
White, Bull. Johns Hopkins Hosp. 1947, 81, 43-54; d) B. Anandaraj, A Vellaichamy, M. Kachman, A. Selvamanikandan, S. Pegu, V. Murugan Biochem. Biophys. Res. Commun. 2009, 379, 179-185; e) C.-D. Qian, X.-C. Wu, Y. Teng, W.-P. Zhao, O. Li, S.-G. Fang, Z.-H. Huang, H.-C. Gao, Antimicrob. Agents Chemother. 2012, 56, 1458-1465; f) X.-C. Wu, C.-D. Qian, H.-H. Fang, Y.-P. Wen, J.-Y. Zhou, Z.-J. Zhan, R. Ding, O. Li, H. Gao, J. Microb. Biotechnol. 2011, 4, 491-502.

[18] H. B. Bode, Curr. Opin. Chem. Biol. 2009, 13, 224-230.

[19] F. Peypoux, M. T. Pommier, D. Marion, M. Ptak, B. C. Das, G. Michel, J. Antibiot. 1986, 39, 636-641.

[20] a) P. Marfey, Carlsberg Res. Commun. 1984, 49, 591-596; b) R. Bhushan H. Brückner, Amino Acids 2004, 27, 231 - 247.

[21] D.-J. Zhang, R.-F. Liu, Y.-G. Li, L.-M. Tao, L. Tian, Chem. Pharm. Bull. 2010 $58,1630-1634$

[22] R. Maget-Dana, F. Peypoux, Toxicology 1994, 87, 151-174.

[23] Y. R. Chung, C. H. Kim, I. Hwang, J. Chun, Int. J. Syst. Evol. Microbiol. 2000, 50, 1495-1500.

[24] K. Tsuge, T. Akiyama, M. Shoda, J. Bacteriol. 2001, 183, 6265-6273.

[25] E. H. Duitman, L. W. Hamoen, M. Rembold, G. Venema, H. Seitz, W. Saenger, F. Bernhard, R. Reinhardt, M. Schmidt, C. Ullrich, T. Stein, F. Leenders, J. Vater, Proc. Natl. Acad. Sci. USA 1999, 96, 13294-13299.

[26] L. Thimon, F. Peypoux, J. Wallach, G. Michel, FEMS Microbiol. Lett. 1995 $128,101-106$

[27] M. H. Zwietering, I. Jongenburger, F. M. Rombouts, K. van 't Riet, Appl. Environ. Microbiol. 1990, 56, 1875-1881.

[28] R. D. Haggett, R. M. Morchat, Int. Biodeterior. Biodegrad. 1992, 29, 87 99.

[29] A. L. Demain, A. Fang, Adv. Biochem. Eng./Biotechnol. 2000, 69, 1-39.

[30] a) F. Campano, J. M. Flores, F. Puerta, J. A. Ruiz, J. M. Ruz in Bee disease diagnosis (Eds.: M. E. Colin, B. V. Ball, M. Kilani), Zaragoza: CIHEAM Options Méditerranéennes, 1999, pp. 61-68; b) K. D. Murray, K. A. Aronstein, J. Microbiol. Methods 2008, 75, 325-328.
[31] M. Kanoaka, A. Isogai, S. Murakoshi, M. Ichjnoe, A. Suzuki, S. Tamura, Agric. Biol. Chem. 1977, 41, 629-635.

[32] a) E. J. Calabrese, L. A. Baldwin, Hum. Exp. Toxicol. 2002, 21, 91-97; b) J. Davies, G. B. Spiegelman, G. Yim, Curr. Opin. Microbiol. 2006, 9, 445453.

[33] R. I. Aminov, Environ. Microbiol. 2009, 11, 2970-2988.

[34] M. A. Wyatt, W. Wang, C. M. Roux, F. C. Beasley, D. E. Heinrichs, P. M. Dunman, N. A. Magarvey, Science 2010, 329, 294-296.

[35] A. Fünfhaus, E. Genersch, Environ. Microbiol. 2012, 4, 194-202.

[36] S. Nordström, I. Fries, J. Apic. Res. 1995, 34, 97-103.

[37] V. M. Markowitz, K. Mavromatis, N. N. Ivanova, I-M. A. Chen, K. Chu, N. C. Kyrpides, Bioinformatics 2009, 25, 2271-2278.

[38] M. Punta, P. C. Coggill, R. Y. Eberhardt, J. Mistry, J. Tate, C. Boursnell, N. Pang, K. Forslund, G. Ceric, J. Clements, A. Heger, L. Holm, E. L. L. Sonnhammer, S. R. Eddy, A. Bateman, R. D. Finn, Nucleic Acids Res. 2012, 40, D290-D301.

[39] K. Blin, M. H. Medema, D. Kazempour, M. A. Fischbach, R. Breitling, E. Takano, T. Weber, Nucleic Acids Res. 2013, 41, W204-12.

[40] M. J. Sullivan, N. K. Petty, S. A. Beatson, Bioinformatics 2011, 27, 10091010.

[41] a) P. W. Okanya, K. I. Mohr, K. Gerth, R. Jansen, R. Müller, J. Nat. Prod. 2011, 74, 603-608; b) R. Jansen, S. Sood, V. Huch, B. Kunze, M. Stadler R. Müller, J. Nat. Prod. 2014, 77, 320-326.

[42] a) P. Aupinel, D. Fortini, H. Dufour, J. N. Tasei, B. Michaud, J. F. Odoux M. H. Pham-Delegue, Bull. Insectol. 2005, 58, 107-111; b) D. J. Lüken, M. Janke, F.-W. Linau, W. von der Ohe, R. Forster, J. Verbraucherschutz Lebensmittelsicherh. 2012, 7, 141-145.

Received: March 29, 2014

Published online on July 28, 2014 


\subsection{Ergänzende Daten zu Kapitel B3}

\section{Inhaltsverzeichnis}

Figure S1-11. ${ }^{1} \mathrm{H},{ }^{13} \mathrm{C},{ }^{1} \mathrm{H}-{ }^{1} \mathrm{H}$ COSY, HSQC, HMBC, ROESY, TOCSY of paenilarvin A in $\mathrm{CD}_{3} \mathrm{OH}$.

Figure S12-13. UV spectrum and ESI HRMS / MS-MS of paenilarvin A.

Figure S14-24. ${ }^{1} \mathrm{H},{ }^{13} \mathrm{C},{ }^{1} \mathrm{H}-{ }^{1} \mathrm{H}$ COSY, HSQC, HMBC, ROESY, TOCSY of paenilarvin B in $\mathrm{CD}_{3} \mathrm{OH}+\mathrm{HCOOH}$.

Figure S25. UV spectrum and ESI HRMS of paenilarvin B.

Figure S26-38. ${ }^{1} \mathrm{H},{ }^{13} \mathrm{C},{ }^{1} \mathrm{H}-{ }^{1} \mathrm{H}$ COSY, HSQC, HMBC, ROESY, TOCSY of paenilarvin B in DMSO-d6.

Figure S39. UV spectrum and ESI HRMS of paenilarvin C.

Figure S40. Toxicity of paenilarvins.

Figure S41. Genetic organization and domain structure of the putative paenilarvin gene cluster.

Abbildungen S1 bis S41 sind auf dem beigefügten, digitalen Medium verfügbar. Dazu bitte den Ordner „Kapitel_B3_Ergänzende_Daten“ öffnen. 


\section{How to kill honey bee larvae: genomic potential and virulence factors of Melissococcus plutonius}

Marvin Djukic ${ }^{1}$, Andreas Leimbach ${ }^{1}$, Daniela Grossar ${ }^{2}$, Jean-Daniel Charrière ${ }^{2}$, Laurent Gauthier $^{2}$, Denise Hartken ${ }^{1}$, Anja Poehlein ${ }^{1}$, Rolf Daniel ${ }^{1}$

${ }^{1}$ Abteilung für Genomische und Angewandte Mikrobiologie \& Göttingen Genomics Laboratory, Institut für Mikrobiologie und Genetik, Georg-August Universität Göttingen, Göttingen, Deutschland

${ }^{2}$ Agroscope, Swiss Bee Research Center, Bern, Switzerland

\section{Anteilserklärung}

Idee/Konzept: JDC, LG, RD

Planung der mikrobiologischen Experimente: MD, AP

Durchführung mikrobiologischer Experimente: MD, DG, DH

Genomsequenzierung und Datenprozessierung: MD, AL, DG, JDC, LG, AP

In silico Genomanalyse: MD

Schreiben des Manuskripts: MD, AL, DG, JDC, LG, DH, AP, RD 


\title{
How to kill honey bee larvae: genomic potential and virulence factors of Melissococcus plutonius
}

\author{
Marvin Djukic', Andreas Leimbach ${ }^{1}$, Daniela Grossar ${ }^{2}$, Jean-Daniel Charrière ${ }^{2}$, \\ Laurent Gauthier $^{2}$, Denise Hartken ${ }^{1}$, Anja Poehlein ${ }^{1}$, Rolf Daniel ${ }^{1 *}$ \\ ${ }^{1}$ Department of Genomic and Applied Microbiology and Göttingen Genomics Laboratory, \\ Institute of Microbiology and Genetics, Georg-August-University Göttingen, Göttingen, \\ Germany
}

${ }^{2}$ Agroscope, Swiss Bee Research Center, Bern, Switzerland

* Correspondence: Rolf Daniel, Genomic and Applied Microbiology and Göttingen Genomics Laboratory, Georg-August University Göttingen, D-37077 Göttingen, Germany Email: rdaniel@gwdg.de

\begin{abstract}
The Gram positive bacterium Melissococcus plutonius is the causative agent of European foulbrood (EFB), a global honey bee disease. The genomes of $14 M$. plutonius strains were analyzed and compared in order to detect potential virulence factors. The genomes exhibited sizes ranging from 2.021 to $2.101 \mathrm{Mbp}$ and comprised 1,595 to 1,686 predicted proteinencoding genes. Comparative genomics revealed genes and predicted proteins that might play a role in EFB pathogenesis such as putative bacteriocins, cell surface and adhesionassociated proteins, an enterococcal polysaccharide antigen, an epsilon toxin, proteolytic enzymes, and capsule-associated proteins. An endo-alpha- $N$-acetylgalactosaminidase, an enhancin and one putative epsilon toxin were verified to be expressed in vivo. Our results showed genomic differences between typical and a highly virulent atypical strain, which indicated that different genomic compositions of $M$. plutonius can contribute to virulence and lead to the death of honey bee larvae. We also detected a high amount of conserved pseudogenes (75 to 156) per genome, which might represent evidence for genomic reduction during evolutionary host adaptation.
\end{abstract}

\section{Introduction}

The Western honey bee (Apis mellifera) is the most important commercial pollinator worldwide (Morse RA, 2000; Aizen et al., 2008; Aizen and Harder, 2009). Approximately $84 \%$ of crop species cultivated in Europe depend on insect pollinators, mainly bees (Gallai 
et al., 2009). Furthermore, honey bees contribute to the pollination of wild plants and are known for their production of economical and medical relevant products such as honey, beeswax, propolis, royal jelly, and apitoxin. European foulbrood (EFB) is one of the major bacterial diseases of honey bees (Forsgren, 2010). The etiological agent of EFB is $M$. plutonius (Bailey, 1957a), a Gram positive, microaerophilic, lanceolate coccus. In general, EFB mainly affects the unsealed brood and honey bee larvae are killed when they are four to five days old (Forsgren, 2010). Infection occurs by ingestion of larval food contaminated with M. plutonius. Subsequently, M. plutonius colonizes the gut of the larvae. Dying larvae are displaced to the wall of the comb cell, often turn yellow and finally take on a brownish color, as they decompose after death (Williams, 2000). In contrast to typical strains, atypical strains display a higher virulence and are not fastidious, meaning that they are able to grow aerobically on some potassium salt-supplemented media, do not require potassium phosphate for growth, show $\beta$-glucosidase activity and hydrolyze esculin (Arai et al., 2012). Furthermore, in contrast to atypical strains, typical M. plutonius strains often lose their virulence after a few cultivation steps in vitro (Bailey, 1957a; Arai et al., 2012), which makes it difficult to investigate virulence factors under laboratory conditions. Until now, virulence factors were not described for M. plutonius and the pathogenicity mechanism still remains unclear.

Here, we present the whole genome sequences of $12 \mathrm{M}$. plutonius strains and a comparative genome analysis with type strain 35311 (Okumura et al., 2011) and atypical strain DAT561 (Okumura et al., 2012). We identified putative virulence factors that might play an important role in EFB pathogenesis. Additionally, we investigated the expression profiles of three putative virulence factors in three EFB-infected larvae. In this way the study contributed to unravel pathogenicity and virulence factors of $M$. plutonius and the underlying molecular mechanisms of EFB.

\section{Materials and Methods}

\subsection{Origin of $M$. plutonius strains}

M. plutonius ATCC 35311 (type strain) was originally isolated in United Kingdom and represents a typical strain according to Bailey and Collins (1982). Strain DAT561 represents an atypical strain and originated from Japan (Okumura et al., 2012). The typical strains 7645B and 765-6B were isolated from EFB outbreaks in Norway and all other typical strains (S1, B5, L9, H6, 21.1, 49.3, 60, 82, 90.0, and 119) from Swiss EFB outbreaks. Isolates B5, H6, and L9 originate from the same Swiss EFB outbreak and the same EFB-infected larva. 
Strains 21.1, 49.3, 60, 82, 90.0, and 119 were isolated from different EFB outbreaks. $M$. plutonius $\mathrm{S} 1$ is a derivate of strain 49.3 and was isolated after five cultivation steps.

\subsection{Growth conditions and isolation of DNA from M. plutonius}

Honey bee larvae with clinical EFB signs were collected from the aforementioned EFB outbreaks in Switzerland and dissected under sterile conditions. Isolates were prepared from diseased larvae mixed with Bailey medium (Bailey, 1957b). Larval smears were streaked on solidified Bailey agar and single colonies picked after anaerobic incubation at $35^{\circ} \mathrm{C}$ for five days. Genomic DNA of $M$. plutonius was extracted of cells in exponential growth phase using the Epicentre MasterPure DNA purification kit (Epicentre, Madison, WI, USA).

\subsection{Genome sequencing, assembly and annotation}

Whole-genome shotgun sequencing of $M$. plutonius strain S1 was performed with a combined sequencing approach using a Genome Analyzer (Illumina, San Diego, CA) and a 454 GS-FLX Titanium XL+ system (GS70 chemistry, Roche Life Science, Mannheim, Germany). 454-shotgun and Illumina Nextera XT shotgun libraries were prepared as recommended by the manufacturers. Sequencing resulted in 149.969 total 454 shotgun reads and 869.292 Illumina 112 bp paired end reads, which were used for a hybrid assembly with MIRA software v3.4.0.1 (Chevreux et al., 1999) resulting in an average coverage of 67x. Editing of the resulting contigs was performed with GAP4, as part of the Staden software package (Staden et al., 2000). Misassembled regions caused by repetitive sequences were resolved and closure of remaining gaps performed by PCR reactions and combinatorial multiplex PCR reactions. Subsequently, Sanger sequencing of the PCR products were performed. Whole-genome shotgun sequencing of M. plutonius isolates H6, L9, B5, 21.1, $60,82,764-5 \mathrm{~B}$, and 765-6B by generating paired-end libraries ( 2 x $112 \mathrm{bp}$ ) with the Nextera XT library preparation kit and employing the Genome Analyzer IIx as recommended by the manufacturer (Illumina, San Diego, CA). Strains 49.3, 90.0 and 119 were sequenced at the Beijing Genomics Institute (Shenzhen, Guangdong, China) with an Illumina Hiseq2000 (Illumina, San Diego, CA) and by employing 90-bp paired-end reads. After read trimming $\begin{array}{lllll}\text { and } & \text { quality } & \text { check } & \text { Trim_Galore } & \text { ving }\end{array}$ (http://www.bioinformatics.babraham.ac.uk/projects/trim_galore/) and FastQC v0.11.2 (http://www.bioinformatics.bbsrc.ac.uk/projects/fastqc/), respectively, a de novo assembly of the paired-end Illumina reads was done with the SPAdes software (Bankevich et al., 2012). Prokka v1.9 (Seemann, 2014) was used for automatic gene prediction and annotation. 
Annotation was afterwards manually curated by employing BLASTP and the Swiss-Prot (Gasteiger et al., 2001), TrEMBL (O'Donovan et al., 2002), and InterProScan 5 database (Jones et al., 2014), and the IMG-ER (integrated microbial genomes-expert review) system (Markowitz et al., 2009). rRNA and tRNA genes were identified with RNAmmer v1.2 (Lagesen et al., 2007) and tRNAscan-SE v1.3.1 (Lowe and Eddy, 1997), respectively. The genome sequences of M. plutonius ATCC 35311 (Accession nr. PRJNA66803) and DAT561 (Accession nr. PRJNA89371) were obtained from NCBI and for comparison the above-described gene prediction and annotation procedures were performed (Supplementary Data 1).

\subsection{Bioinformatic genome analyses}

The sequence type (ST) of $M$. plutonius strains were determined by multilocus sequence typing (MLST) (Larsen et al., 2012) according to the protocol of Haynes et al. (2013). The sequence type of each strain was determined in silico using the public available ST data from the PubMLST database (as of $23^{\text {th }}$ June 2015) (http://pubmlst.org/). The program PHYLOViZ v1.1 (Francisco et al., 2012) was used to calculate and visualize the minimum spanning tree (MST) composed of sequence types (ST) and clonal complexes (CC). Orthologous proteins were identified with the program Proteinortho v5.11 (parameters: identity cutoff $50 \%$, coverage cutoff $50 \%$, e-value cutoff for blastp 1e-05) (Lechner et al., 2011) by using the CDS (coding sequences) protein sequences deduced from the 14 Melissococcus genomes as input. For this purpose, cat_seq v0.1 and cds_extractor v0.6 were used (Leimbach 2014). Based on this data presence and absence of orthologous groups was converted into a simple binary matrix and a gene content tree was calculated via RAxML v8.1.3 (Stamatakis, 2014) with 1000 bootstrap resamplings and GAMMA model of rate heterogeneity. For visualization, the Dendroscope software v3.2.1 (Huson and Scornavacca, 2012) was used.

Harvest v1.1.2 together with Parsnp v1.2 and Gingr v1.2 as part of the Harvest software suite (Treangen et al., 2014) were used to perform core genome alignment, calculate genome phylogeny, and identify and visualize single nucleotide polymorphisms (SNPs) and short insertions and deletions (Indels). M. plutonius 49.3 was used as the reference strain. The Phage Search Tool (PHAST) (Zhou et al., 2011) was used to determine prophage sequences within the bacterial genomes.

GIPSy software v1.1.1 (http://www.bioinformatics.org/ftp/pub/gipsy/) was used to detect genomic islands (parameters: $\mathrm{G}+\mathrm{C}$ content analysis cutoff value of 1.5 standard deviations, 
sensitivity to calculate codon usage deviation of 0.95 , e-value of $1 \mathrm{e}-5$ for transposases prediction using HMMer, e-value of $1 \mathrm{e}-10$ for prediction of pathogenicity islands via virulence factor detection using blastp, reciprocal blastp analysis between the query and subject genome and the tRNA prediction using HMMer were done using an e-value of 1e10 and 1e-5, respectively, and $M$. plutonius ATCC 35311 was set as query). Additionally, a blastp (e-value 1e-50) of the CDS protein sequences against the virulence factor database (VFDB) (Chen et al., 2012) was performed to detect putative virulence factors. The detection of putative bacteriocins was done via BAGEL3 (van Heel et al., 2013), Bactibase (database as of $17^{\text {th }}$ Feb. 2015) (Hammami et al., 2010), and IMG-ER (Markowitz et al., 2009). The MEROPS database v9.12 (Rawlings et al., 2014) was used to detect proteolytic enzymes and their substrates.

We used BRIG v0.95 (Alikhan et al., 2011) and easyFig v2.1 (Sullivan et al., 2011) to visualize whole genome and genome region comparisons, respectively.

\section{5. cDNA synthesis and reverse transcription PCR (RT-PCR)}

Three $5^{\text {th }}$ instar worker larvae, Apis mellifera, displaying EFB symptoms were sampled from the same beehive in Lützelflüh, Switzerland, in June 2013. Furthermore, one healthy honey bee $5^{\text {th }}$ instar larva was sampled from Lohne, Germany, in August 2014 and used as negative control. Sampled larvae were frozen in liquid nitrogen immediately after collection. Honey bee larvae were individually homogenized in sterile TE buffer (10 mM Tris- $\mathrm{HCl}$ (pH 7.4), $1 \mathrm{mM}$ EDTA) supplemented with $3 \mathrm{mg} / \mathrm{ml}$ lysozyme. $50 \mu \mathrm{l}$ of the homogenate were used for a fast EFB confirmation test using the Vita EFB Diagnostic Test kit (Vita Europe, Basingstoke, Great Britain) and $10 \mu \mathrm{l}$ were used for a colony PCR with specific primer pairs targeting the 16S rRNA gene of M. plutonius (Govan et al., 1998) (Supplementary Table 1). Subsequently, parallel isolation of total DNA and RNA was performed by using the DNeasy Blood and Tissue kit and RNeasy Mini kit supplemented with RNAprotect, respectively, as recommended by the manufacturer (Qiagen, Hilden, Germany).

RNA extracts were treated with DNase I (Thermo Scientific, Germany) and purified with RNeasy MinElute CleanUp kit (Qiagen, Hilden, Germany). Complete removal of DNA was verified by a PCR reaction targeting the $16 \mathrm{~S}$ rRNA gene using a specific primer pair (16S08F / 16S-1504R) (Supplementary Table 1). The DreamTaq DNA Polymerase was used as recommended by the manufacturer (Thermo Scientific, Germany). Purified RNA was transcribed to single strand cDNA (sscDNA) using the QuantiTect Reverse Transcription kit 
(Qiagen, Hilden, Germany). The resulting ssDNA was used for RT-PCRs of single genes of M. plutonius directly.

Transcription of $M$. plutonius specific genes in EFB-infected larvae were tested using RTPCR (primers are presented in Supplementary Table 1). These included the putative virulence factors endo-alpha- $N$-acetylgalactosaminidase, enhancin, and a toxin. The transcriptions of the $16 \mathrm{~S}$ rRNA gene, rpoD (RNA polymerase sigma factor), and rho (transcription termination factor) were used as positive controls. Genomic DNA of strain 49.3 was used as positive control and strain S1 as negative control as the toxin gene is not present in this strain. For amplification of the 16S rRNA gene, genomic DNA and cDNA from a healthy honey bee larva was used as negative control. All PCRs were performed using the Bioxact kit as recommended by the manufacturer (Bioline, London, United Kingdom).

\subsection{DNA sequences obtained and GenBank submissions}

The genome sequences reported in this paper have been deposited in the GenBank database under accession numbers JSAY00000000 (M. plutonius 21.1), JSBA00000000 (M. plutonius 49.3), JSBE00000000 (M. plutonius 60), JSBF00000000 (M. plutonius 82), JSAZ00000000 (M. plutonius 90.0), JSBB00000000 (M. plutonius 119), CP006683-CP006684 (M. plutonius S1), JSAW00000000 (M. plutonius B5), JSBC00000000 (M. plutonius H6), JSBD00000000 (M. plutonius L9), JSAV00000000 (M. plutonius 764-5B), JSAX00000000 (M. plutonius 765-6B).

\section{Results}

\subsection{MLST of M. plutonius EFB isolates}

In order to analyze the molecular epidemiology and population structure of M. plutonius and unravel the phylogenetic relationship of 12 isolated strains from Switzerland and Norway, we assigned sequence types (STs) to all isolated strains. The sequences of four genes ( $\mathrm{galK}$, $\arg E, g b p B$, and $p u R$ ) were determined according to the M. plutonius MLST protocol of Haynes et al. (2013). Based on the STs clonal complexes (CC) were calculated as single locus variants (Figure 1). Four isolates belong to ST3 (49.3, S1, 764-5B, and 765-6B) and five isolates belong to ST7 $(21.1,60, \mathrm{~B} 5, \mathrm{H} 6$, and L9) grouped into CC3. Two strains originated from Norway (764-5B and 765-6B) and all other strains from Switzerland (Supplementary Table 2).

The remaining M. plutonius 119 and strain 90.0 belong to ST20 and ST13, respectively, and their corresponding STs are part of CC13 (Supplementary Table 2). Interestingly, the ST 
profile from $M$. plutonius 82, another isolate from Switzerland, with $\operatorname{argE~} 1, \operatorname{galK} 8, \operatorname{gbpB}$ 2, and purR 4 could not be assigned to an already existing ST. Thus, a novel ST, ST32, was defined within CC13.

No strain could be allocated to the known $\mathrm{CC} 12$ to which the atypical strain from Japan belongs. The type strain M. plutonius ATCC 35311 from England was assigned to ST1 (CC13) according to Haynes et al. (2013).
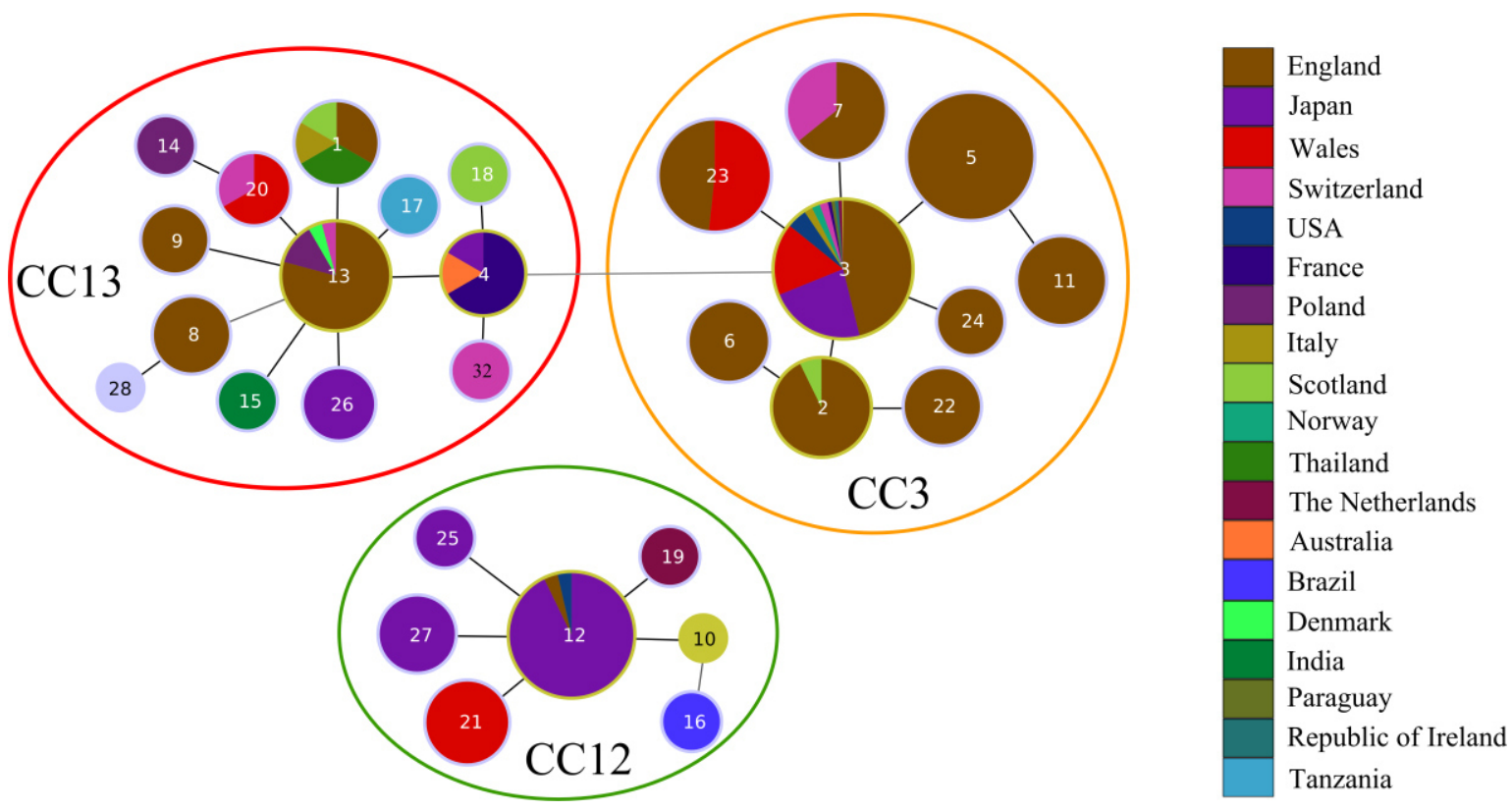

Figure 1: Minimum spanning tree of sequence types (ST) found in M. plutonius isolates from different countries. MLST data obtained from this study was added to those reported previously (Takamatsu et al. 2014) and resulted in three clonal complexes (CC3, CC12, and CC13). Altogether 364 isolates were used. Each circle represents a different ST, and the size indicates the frequency of occurrence. Closest relatives are linked with lines. Black lines indicate a single allelic variant between STs, gray lines variation at two loci. Colors within circles represent the proportion of isolates of a particular ST that were found in the countries indicated on the right. The obtained data was submitted to PubMLST.

\subsection{Genome analysis}

\subsubsection{General genome properties}

The genomes of the typical strains M. plutonius 21.1, 49.3, 60, 82, 90.0, 119, B5, H6, L9, $\mathrm{S} 1,764-5 \mathrm{~B}$, and 765-6B range from 2.021 to 2.101 Mbp and comprise between 1,589 and 1,686 predicted protein-coding genes (Supplementary Table 2). For comparison, the genome sequences of the typical strain M. plutonius ATCC 35311 and atypical strain DAT561 were derived and treated in the same way as that from the isolates. Gene content comparisons were performed by using the genome $M$. plutonius 49.3 as reference (Figure 2). In general, the genomes are very similar in their gene content, except for a plasmid pMP19 that is 
missing in nine typical strains $(82,90.0,119, \mathrm{~B} 5, \mathrm{~L} 9, \mathrm{~S} 1,764-5 \mathrm{~B}, 765-6 \mathrm{~B}$, ATCC 35311) and the atypical strain DAT561. Based on this results, a phylogenetic tree was obtained via the Harvest software suite (Treangen et al., 2014) through a core-genome alignment with SNP detection (Figure 3). This phylogeny can resolve the relationships of the strains in more detail than MLST analysis. It confirms the close relationship between ST3 and ST7 strains, but shows that $M$. plutonius 82 is actually a sister taxon of M. plutonius ATCC35311 within the monophyletic lineage of CC13 strains. Interestingly, a high amount of putative pseudogenes could be detected (between 75 to 156) (Supplementary Table 2). Pseudogenes emerged mostly due to small mutational events in coding regions like SNPs and Indels, which lead to frameshifts and premature stop codons (Tutar, 2012).

All strains harbor a plasmid with high DNA sequence similarity to the recently published plasmid pMP1 (NC_015517) of M. plutonius ATCC 35311. Moreover, several strains such as 21.1, 49.3, 60, B5, and H6 might contain additional plasmids. Strain B5 harbors a 42.7 kbp plasmid (pMP43) encoding phage proteins, which are also present in the chromosome of B5, H6, L9, 49.3, S1, 60, 21.1, 764-5B, and 765-6B (see prophage region 1, Figure 2). In addition, we identified a 19.4-kbp plasmid (pMP19) present in strain 49.3. Contigs of strains 21.1, 60 and H6 show high sequence similarity to this plasmid, indicating that it is also present in these strains.

Through the identification of orthologous proteins we calculated the core genome of this set of M. plutonius strains to 1304 proteins, which represents on average approx. $71 \%$ of the proteins encoded by a $M$. plutonius genome. The pan genome has a total of 1846 proteins. 


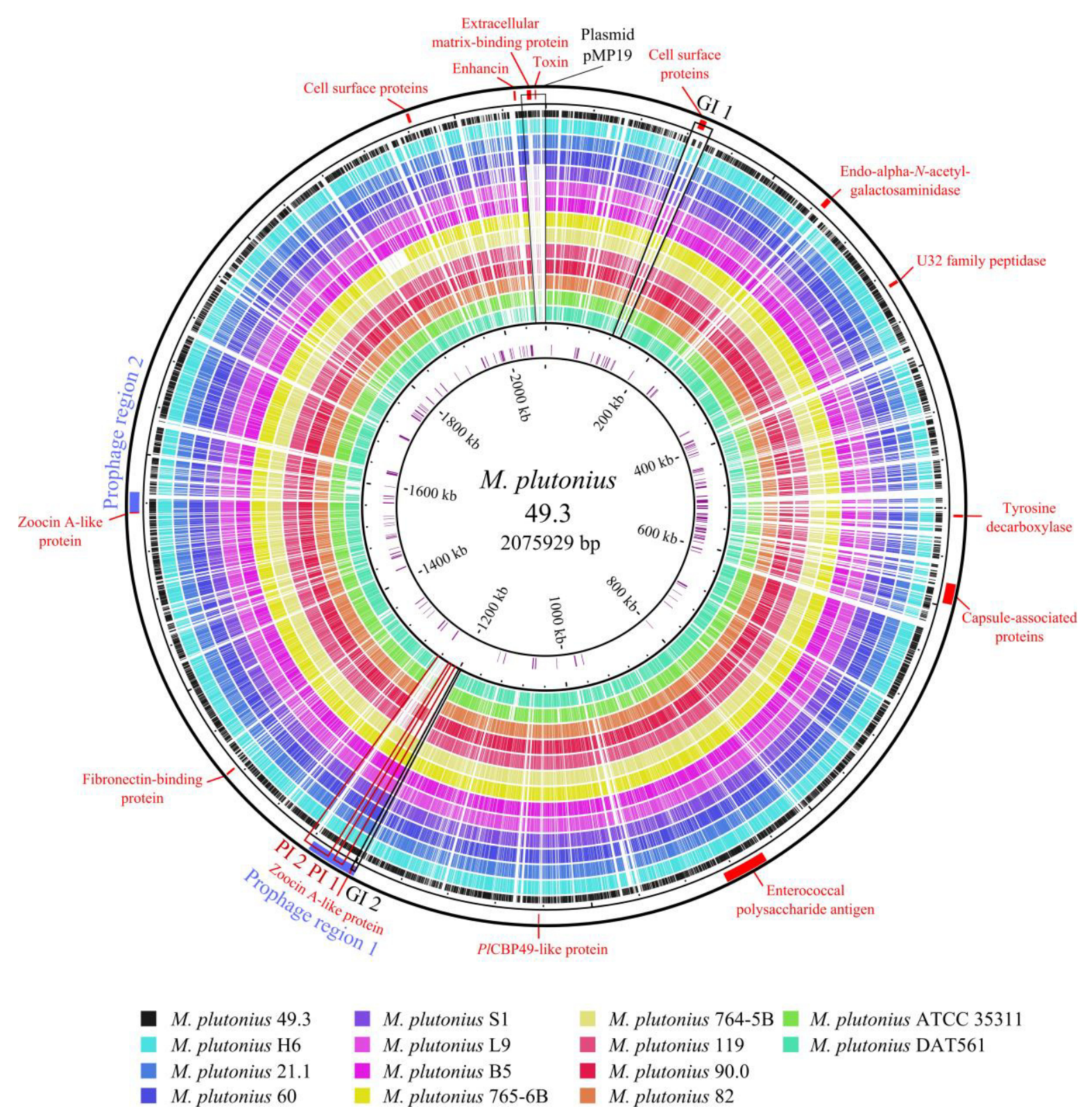

Figure 2: M. plutonius 49.3 artificial circular genome map. Comparison of the M. plutonius 49.3 genome to the genomes of strains H6, 21.1, 60, S1, L9, B5, 765-6B, 764-5B, 119, 90.0, 82, ATCC 35311, and DAT561 using the BRIG software (Alikhan et al., 2011). The inner circle shows the positions of pseudogenes in the M. plutonius 49.3 genome, while virulence factors and prophage regions are depicted on the outer circle and marked with red and blue blocks, respectively. Furthermore, pathogenicity (PI) and genomic islands (GI) are encircled and numbered in red and black. The plasmid pMP19 of M. plutonius 49.3 is indicated as well. 


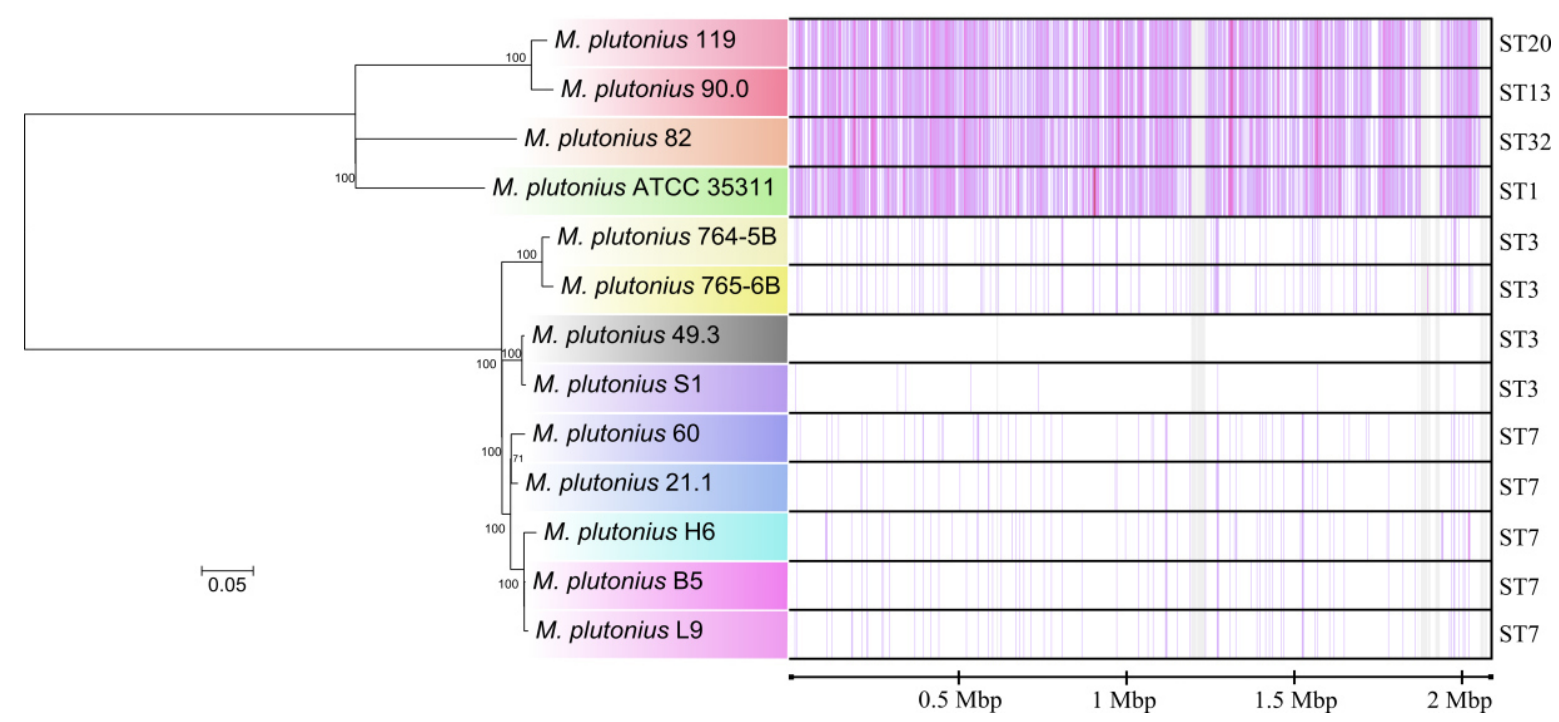

Figure 3: Phylogenetic tree based on core-genome SNP-typing of typical M. plutonius strains used in this study. The phylogenetic tree on the left was obtained via the Harvest software suite (Treangen et al., 2014) and is based on a core-genome SNP typing. M. plutonius 49.3 was set as the reference strain. The strains are marked in the same color code used in Figure B. The SNP and Indel positions in relation to the reference are shown on the right hand side as violet lines. Sequence types are shown as well for comparison purposes.

\subsubsection{Detection of putative virulence factors}

In their assay, Arai et al. (2012) showed that atypical strains are more virulent than typical strains. The atypical strain DAT561 was shown to kill 94\% of EFB-infected honey bee larvae five days after infection while in contrast, typical strains killed approx. $6 \%$ of the EFBinfected larvae within this timeframe. In order to correlate the functional capabilities of the strains to their phylogeny, we calculated a gene content tree based on the presence and absence of proteins in each strain (Figure 4). The gene content tree showed high similarity to the SNP-based phylogeny. The atypical strain DAT561 formed an outgroup while ST3/ST7 strains cluster together. Interestingly, the typical strains originate from different phylogenetic cluster (Figure 3). Therefore, we focused our study on the identification of $M$. plutonius putative virulence factors.

Atypical and typical strains have phenotypic differences (Arai et al., 2012) and Takamatsu et al. (2014) suggested different regulation mechanisms for virulence. To verify these differences on a genomic level, orthologous proteins integrated in all strains were analyzed. We obtained 132 proteins, which are present in the atypical strain but are absent in all typical strains (Supplementary Data 2, Sheet 1a, Figure 4). Some of these orthologous proteins potentially represent virulence factors or variations in metabolic properties necessary for a pathogenic lifestyle. Typical M. plutonius strains have 275 orthologs in common, which are absent in the atypical strain DAT561 (Supplementary Data 2, Sheet 1b). The majority of 
these orthologs are hypothetical or phage-related proteins but several putative virulence factors could be identified. In addition, putative virulence factors were determined by identifying genomic and pathogenicity islands and their associated virulence determinants (Supplementary Data 2, Sheet 2, Sheet 3a, and Sheet 3b). A summary of all identified putative virulence factors is depicted in Supplementary Data 2, Sheet 4. Putative virulence factors include a tyrosine decarboxylase, bacteriocins, PlCBP49-like protein, enhancin, collagenase, cell surface and adhesion-associated proteins, capsule and antigen-forming proteins, and a toxin.

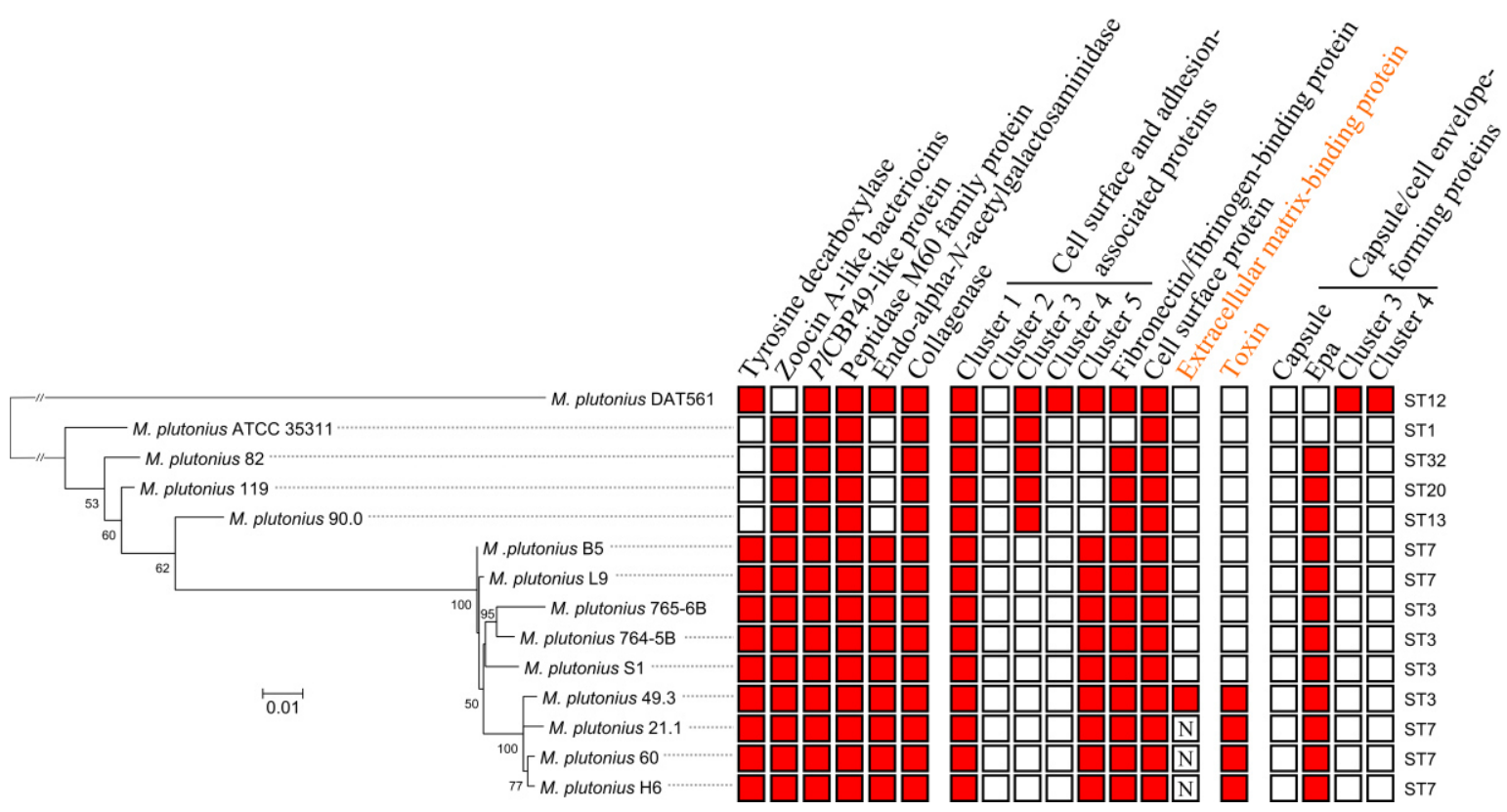

Figure 4: Gene content tree based on presence or absence of orthologous proteins. For constructing the phylogenetic tree a presence/absence binary matrix was created from orthologous groups to calculate a phylogeny with RAxML v8.1.3 (Stamatakis, 2014). M. plutonius DAT561 was used as an outgroup. Numbers at nodes are bootstrap values calculated from 1,000 resamplings to generate a majority consensus tree. The scale bar indicates divergence in presence or absence of proteins. STs are shown on the right. Color-filled boxes to the right of the organisms show the presence of the indicated proteins. Genes encoding virulence factors in brown are located on pMP19. An "N" symbolizes that the respective ORF is not complete due to a gap in the DNA sequence. Epa is the abbreviation for "enterococcal polysaccharide antigen".

\subsection{Identification and analysis of putative virulence factors}

\subsubsection{Tyramine}

Kanbar et al. (2004) showed that tyramine production of E. faecalis has highly toxic effects on honey bee larvae (Kanbar et al., 2005). Furthermore, the development of tyramine treated honey bee brood was impaired and showed classical EFB symptoms as treated larvae changed their color to yellow/brown. We identified an Enterococcus-type tyrosine decarboxylase gene cluster, which is involved in tyramine production (Connil et al., 2002). 
Interestingly, the genes encoding the tyrosine decarboxylase of the typical strains 82, 90.0, 119 and ATCC 35311 are putatively dysfunctional due to a nonsense mutation (Supplementary Data 2, Sheet 4).

\subsubsection{Bacteriocins}

A high number of bacteria produce peptides called bacteriocins, which possess antimicrobial activities against very close related species or even against strains of the same species (Zacharof and Lovitt, 2012). A total of seven (typical strains) or five (atypical strain) genes and gene clusters encoding for putative bacteriocin biosynthesis and transport functions were identified in the genomes of the typical strains and the atypical strain DAT561 (Supplementary Data 2, Sheet 4). These clusters share high similarity to putative bacteriocin biosynthesis clusters of Enterococcus and transport clusters of Streptococcus spp. (Supplementary Figure 1). Here, we only focus on putative functional genes encoding for bacteriocin biosynthesis proteins, although the functionality of most of the gene clusters is uncertain due to nonsense mutations in the corresponding ORFs (Supplementary Data 2, Sheet 4). Two putative functional ORFs share low similarity with Zoocin A-like bacteriocins (Supplementary Data 2, Sheet 4, see "Bacteriocin-associated proteins", ORF1 and 2), and one with an unclassified bacteriocin determined by BAGEL3 (van Heel et al., 2013) (Supplementary Data 2, Sheet 4, see "Bacteriocin-associated proteins", ORF3). ORF1 and ORF2 share $14 \%$ and $18 \%$ amino acid sequence identity ( $31 \%$ and $39 \%$ coverage) to Zoocin A, respectively, a streptococcolytic enzyme with weak $\beta$-lactamase activity (Heath et al., 2004). Remarkably, ORF1 is only present in typical strains of ST3 and ST7. On the contrary, ORF3 was found in all other ST determined in this study, excluding the atypical strain. Thus, putative bacteriocin biosynthesis proteins were only identified in typical strains. In addition, we found lysozyme subfamily 2 domain/GH73 family domain-containing proteins (Supplementary Data 2, Sheet 4), which might be involved in bacterial cell wall degradation (Joris et al., 1992).

\subsubsection{Larval glycoprotein and peritrophic matrix degrading enzymes}

The peritrophic matrix lines the midgut of invertebrates and is comprised of secreted chitin and (glyco)proteins, mainly peritrophins (Terra, 2001). It compartmentalizes digestive processes, protects from ingested xenobiotics, and acts as a mechanical barrier against abrasive food pieces and pathogens (Garcia-Gonzalez et al., 2014c; Terra, 2001). In the genomes of $M$. plutonius a potential chitin-binding domain-containing protein, consisting of 
a signal peptide and a type 3 chitin binding domain, was identified (Supplementary Data 2, Sheet 4). It belongs to the auxiliary activity 10, a family of lytic polysaccharide monooxygenases, and exhibited $37 \%$ amino acid sequence similarity to PlCBP49 (JX185746) of $P$. larvae. PlCBP49 represents a key virulence factor of $P$. larvae and is able to degrade the peritrophic matrix of the honey bee larva (Garcia-Gonzalez et al., 2014c). Additionally, a peptidase M60 family protein (enhancin), which can potentially degrade the peritrophic matrix (Toprak et al., 2012; Tellam et al., 1999; Fang et al., 2009; Peng et al., 1999) of the honey bee larvae, was detected (Figure 4, Figure 5, and Supplementary Data 2, Sheet 4). It contains a signal peptide and shows high similarity to an enhancin-like protein of Bacillus thuringiensis serovar kurstaki str. YBT1520 (51\% identity, ACN22337) (Figure 5). The latter was shown to disrupt the insect midgut peritrophic matrix (Fang et al., 2009). The peptidase M60 family protein also shows low amino acid sequence similarity to a M60 family protein of P. larvae DSM 25719 (22\% identity, ERIC1_1c29890) (Djukic et al., 2014). Additionally, it is homologous to several pseudogenes of P. larvae DSM 25719 and P. larvae DSM 25430, which are fragmented by transposase insertions or mutations (Figure 5) and putatively dysfunctional. The typical $M$. plutonius strains harbor an identical enhancin protein (744 amino acids), whereas the enhancin of atypical strain DAT561 is slightly truncated (728 amino acids).

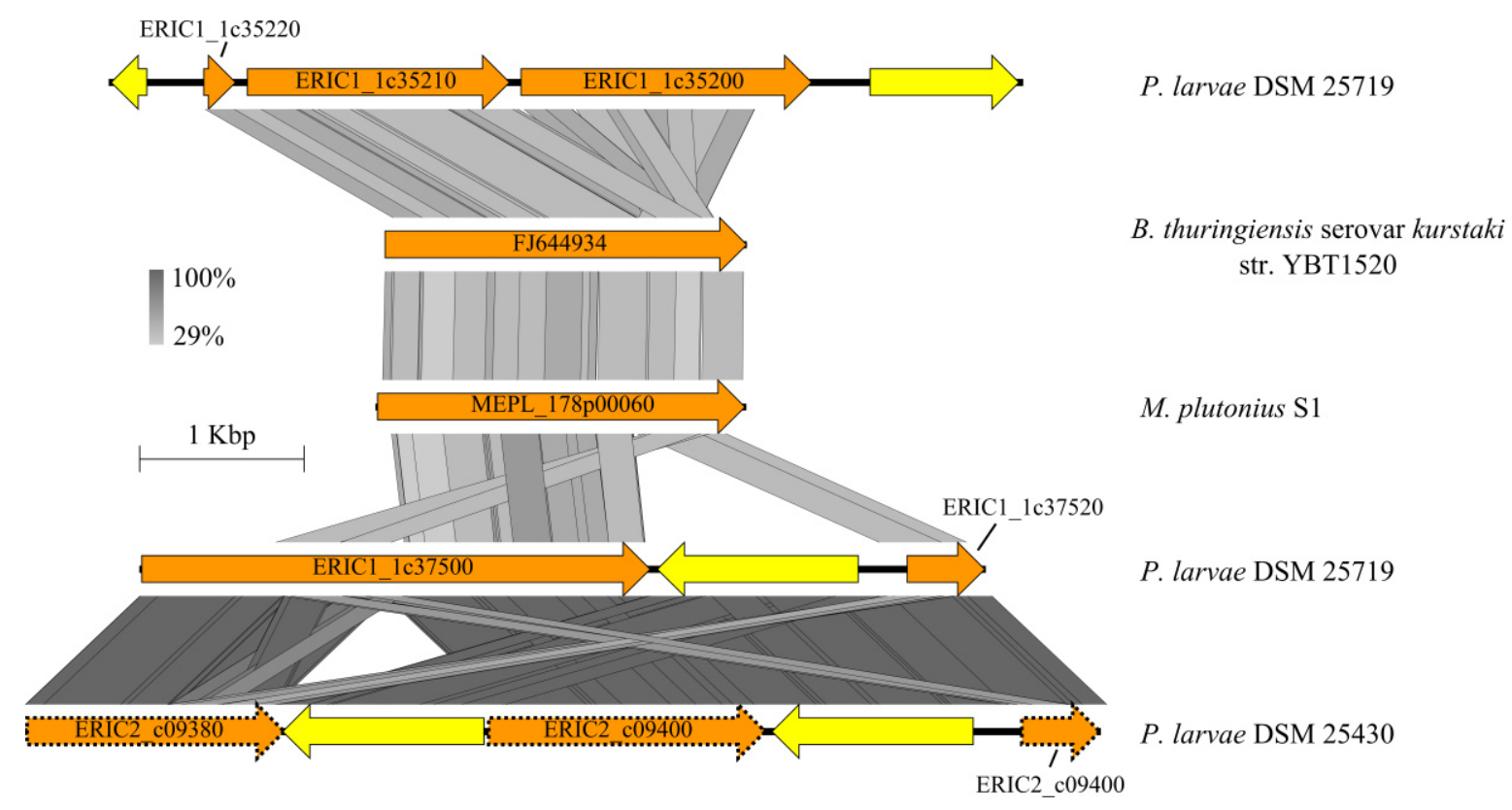

Figure 5: Comparison of the enhancin gene cluster of M. plutonius S1 with P. larvae DSM 25719, $P$. larvae DSM 25430 and B. thuringiensis serovar kurstaki str. YBT1520. The graphical presentation was done with the Easyfig software (minimum blast hit length of $50 \mathrm{bp}$ ) (Sullivan et al., 2011). ORFs depicted as dotted arrows represent pseudogenes. ORFs related to enhancin are orange and transposases are shown in yellow. 
Furthermore, we detected a gene encoding putative endo-alpha- $N$-acetylgalactosaminidase (EC 3.2.1.97) that catalyzes the release of oligosaccharides via hydrolysis of the $O$ glycosidic bond between alpha-acetylgalactosamine at the reducing end of mucin-type sugar chains (O-glycan) and serine/threonine residues of proteins, which is putatively dysfunctional in the M. plutonius strains 119, 82, 90.0 and ATCC 35311 due to nonsense mutations. As shown in Supplementary Figure 2, the peptidase M60 family protein as well as the endo-alpha- $N$-acetylgalactosaminidase are transcribed in vivo during $\mathrm{EFB}$ pathogenesis, while an expression of these putative virulence factors was not detected in a healthy honey bee larva (data not shown).

\subsubsection{Cell surface and adhesion-associated proteins}

The ability to adhere to extracellular matrix proteins of animal cells like fibronectin, fibrinogen, collagen, and laminin is the first and critical step to establish an infection for many pathogenic bacteria (Courtney et al., 1994; Holmes et al., 2001; Massey et al., 2001; Spigaglia et al., 2013). Altogether five gene clusters and three single ORFs were associated with cell surface and adhesion and putatively have an impact on virulence. Each typical strain has nonsense mutations in at least one of the cluster involved in adhesion (Supplementary Data 2, Sheet 4). An overview of the identified cell surface and adhesionassociated proteins including their domain structures is depicted in Figure 6 and the presence and absence of selected proteins is shown in Figure 4 and Supplementary Data 2, Sheet 4. Interestingly, the genomes of the typical strains encode less potentially functional cell surface and adhesion-associated proteins than the atypical strains DAT561. Two gene clusters (one and five) of the typical strains are putative remnants of clusters detected in the atypical strain DAT561, cluster three contains one ORF with a nonsense mutation and cluster four is missing in all typical strains (Figure 4).

A fibronectin/fibrinogen-binding domain (DUF814)-containing protein was discovered in all strains used in this study (Figure 4 and Figure 6). The corresponding ORF encodes a protein, which shares high similarity (70\% identity and $99 \%$ coverage) to the fibronectinbinding protein of Enterococcus caccae and E. moraviensis (WP_010772361 and WP_010765067, respectively). Fibronectin and fibrinogen are essential parts of the extracellular matrix of animal cells. Thus, many bacterial pathogens harbor proteins for adhesion involving these proteins, e.g. FbpA, a surface fibronectin-binding protein required for intestinal and liver colonization of Listeria monocytogenes (Dramsi et al., 2004). A putative extracellular matrix-binding protein (MEPL7_19p00060, Figure 4 and Figure 6) is 
plasmid-encoded (pMP19) and only present in the typical strains 21.1, 49.3, 60 and H6 (Supplementary Data 2, Sheet 4). It contains eight copies of a DUF1542 domain. In Staphylococcus aureus it was shown that some DUF1542-containing proteins are involved in cell cluster formation, cellular adhesion and antibiotic resistance (Clarke et al., 2002; Schroeder et al., 2009). This protein shares the highest amino acid sequence identity (47\%) with a matrix-binding protein of Lactobacillus rhamnosus (WP_033571521).

Melissococcus plutonius 49.3
(typical strain)

MEPL7_7c00660 MEPL7_7c00650

Cluster 1

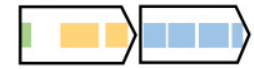

Cluster 2

MEPL7 1 01020

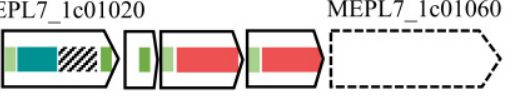

Cluster 3
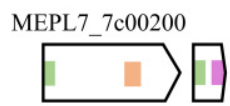

MEPL7 7c00230

Cluster 4

MEPL7_1c02050

MEPL7_1c02070

MEPL7_1c02080

Cluster 5

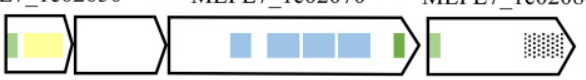

Fibronectin/fibrinogen

-binding protein

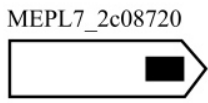

MEPL7_2c02580

Cell surface protein

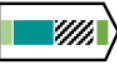

Melissococcus plutonius DAT561

(atypical strain)
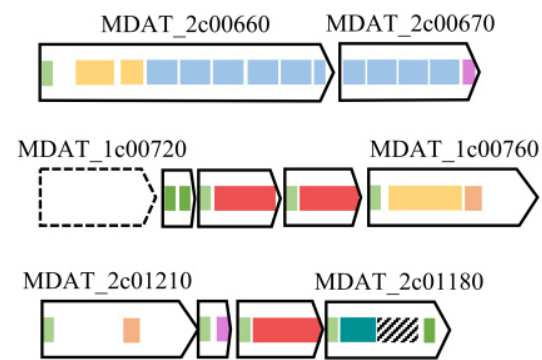

MDAT 2c00950 MDAT 2c00960 MDAT_2c01120 MDAT_2c01110
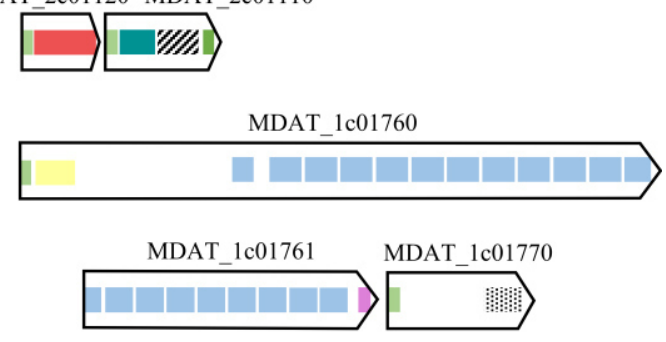

MDAT $1 \mathrm{c} 07560$

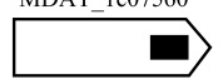

MDAT_1c12950

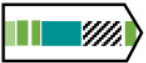

Extracellular matrixbinding protein

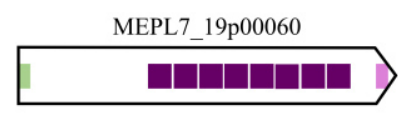

WxL domain (IPR027944)

Bacterial Ig-like domain (IPR022038)

DUF1542 (IPR011439)

Adhesion domain (IPR008966)

CAP domain (IPR014044)

DUF814 (IPR008532)

Figure 6: Domain structure of putative cell surface and adhesion proteins identified in M. plutonius 49.3 and DAT561 with a putative role as virulence factors. Signal peptides, transmembrane regions and domains were determined using InterProScan 5, and are depicted using the color code shown in the legend. Cluster sizes range from 2 to $5.3 \mathrm{kbp}$ in M. plutonius 49.3 and $1.7 \mathrm{kbp}$ to $9.8 \mathrm{kbp}$ in $M$. 
plutonius DAT561. The presence of orthologous genes and gene cluster identified in the other strains are shown in Figure 4 and Supplementary Data 2, Sheet 4.

\subsubsection{Toxin}

In addition to M. plutonius, Paenibacillus larvae causes also an important bacterial disease afflicting the honey bee brood (American foulbrood (AFB)). In a recent review, it was shown that $P$. larvae attacks honey bee larvae epithelial cells via secreted toxins during pathogenesis (Poppinga and Genersch, 2015). These P. larvae-toxins destroy the epithelial integrity and enable bacteria to breach the epithelium of larvae via a paracellular route. Only the genomes of the typical M. plutonius strains 21.1, 49.3, 60 and H6 harbor a putative toxinencoding ORF (Supplementary Data 2, Sheet 4), while all other typical strains and the atypical strain DAT561 lack a respective gene. The toxin, now designated "melissotoxin A", is plasmid-encoded (pMP19). It shows 33\% amino acid sequence identity to an epsilon toxin ETX/mosquitocidal toxin MTX2 family protein of Brevibacillus laterosporus (WP_018669999), a common secondary invader in EFB disease (Djukic et al., 2011). The putative toxin harbors an N-terminal signal peptide and is similar to proteins of PFAM family PF03318 such as the Clostridium epsilon toxin ETX and the Bacillus mosquitocidal toxin MTX2, and Aerolysin-like toxins. Noteworthy, the melissotoxin A-encoding gene is expressed during infection in vivo (Supplementary Figure 2).

\subsubsection{Capsule/cell envelope-forming proteins}

Capsules are a layer of surface-associated polysaccharides. They protect the bacteria against desiccation, attack from phages, antimicrobial peptides, and sometimes from phagocytosis (Schembri et al., 2004; Campos et al., 2004). We detected four gene clusters, which are associated with capsule and cell envelope-forming proteins (Supplementary Data 2, Sheet 4).

Gene cluster 1 comprises a putative capsule locus, which was described for E. faecium strains by Palmer et al. (2012). The putative capsule-encoding gene cluster of E. faecium 504 and E. caccae ATCC BAA-1240 share high sequence similarity to this cluster, although all Melissococcus strains contain nonsense mutations in genes involved in capsule formation (Supplementary Data 2, Sheet 4). The number of putatively non-functional ORFs due to nonsense mutations varies between one (strain DAT561) and three to four (all typical strains).

The second gene cluster has a similar composition as the enterococcal polysaccharide antigen (epa)-locus of E. faecalis (Xu et al., 1997; Hancock et al., 2012; Xu et al., 1998), E. 
haemoperoxidus and E. caccae (Figure 7). A role of Epa as virulence factor is suggested at least for E. faecalis (Hancock et al., 2012). Furthermore, Epa facilitates resistance to bile salts and antimicrobial peptides (Rigottier-Gois et al., 2014).M. plutonius ATCC 35311 and the atypical strain DAT561 are the only strains, which have frameshift mutations in at least one gene of this cluster (Supplementary Data 2, Sheet 4).

Cluster three and four consist of two ORFs each, which both are putatively only functional in the atypical strain DAT561 (Supplementary Data 2, Sheet 4). ORFs belonging to these clusters encode lipid A-like transporters.

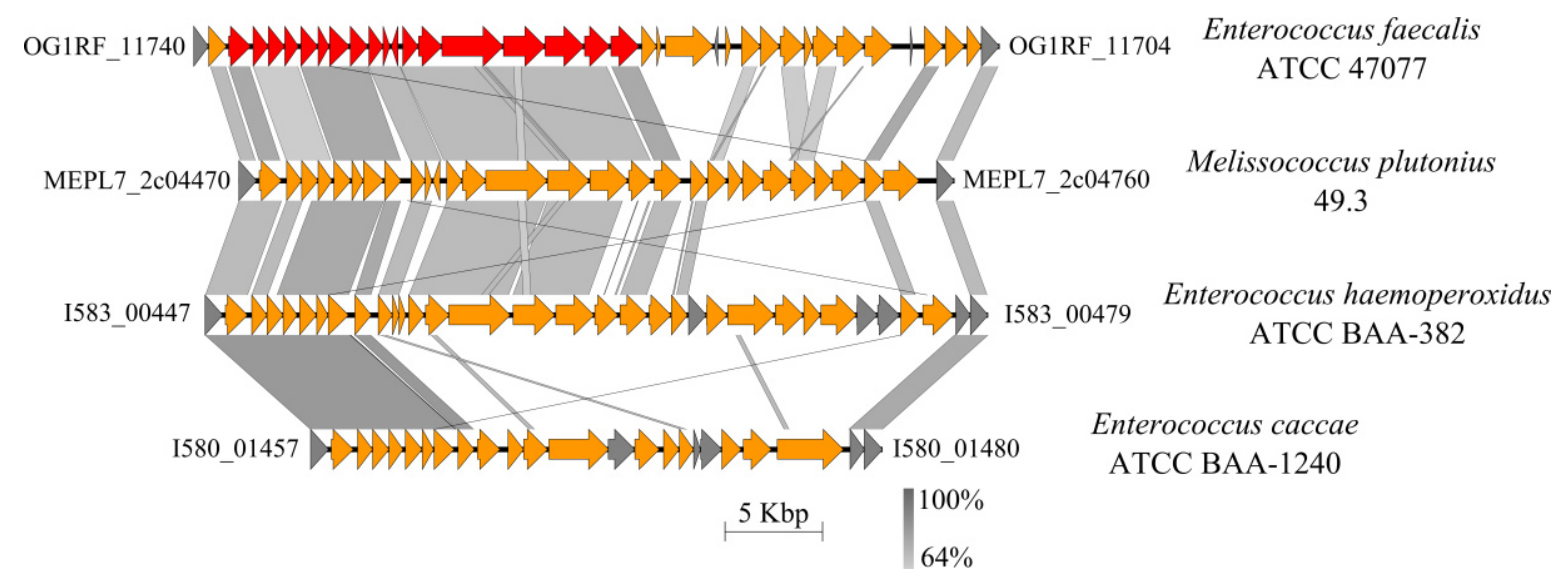

Figure 7: Comparison of a gene cluster of M. plutonius with gene clusters of Enterococci encoding for Epa. ORFs labeled with locus tags represent the corresponding ends of the shown genome segments. ORFs related to the epa-locus are marked in orange and ORFs encoding Epa of E. faecalis are depicted in red. Conserved hypothetical proteins are shown in gray. The gene cluster shows highest sequence similarity to Epa of E. faecalis and E. haemoperoxidus.

\subsection{Energy and sugar metabolism}

Competition for resources with the host results in evolutionary pressure on bacteria. For this reason, we studied in more details the potential pathways for energy and sugar metabolism in M. plutonius. All M. plutonius strains lack a tricarboxylic acid cycle (TCA) and the electron transport system for oxidative phosphorylation. Enzymes for a glycolysis system were found in all strains, but the genes encoding pyruvate kinase and transketolase of the atypical strain DAT561 are interrupted by frameshift mutations. Enzymes required for homolactic acid fermentation were identified, but a glucose-6-phosphate dehydrogenase, a 6-phosphogluconolactonase, and a decarboxylating 6-phosphogluconate dehydrogenase as part of the heterolactic acid fermentation are also encoded. An overview about glycolysis, the pentose phosphate pathway, the Entner-Doudoroff pathway, mixed acid fermentation, sugar interconversions (partly) and pyruvate metabolism of $M$. plutonius is shown in Figure 
8. Additionally, amino acid decarboxylation and the arginine deiminase pathway can contribute to energy production.

Furthermore, we detected a number of genes encoding enzymes that target plant cell wall polysaccharides as described for the honey bee gut microbiota (Engel et al., 2012) (Supplementary Figure 3).

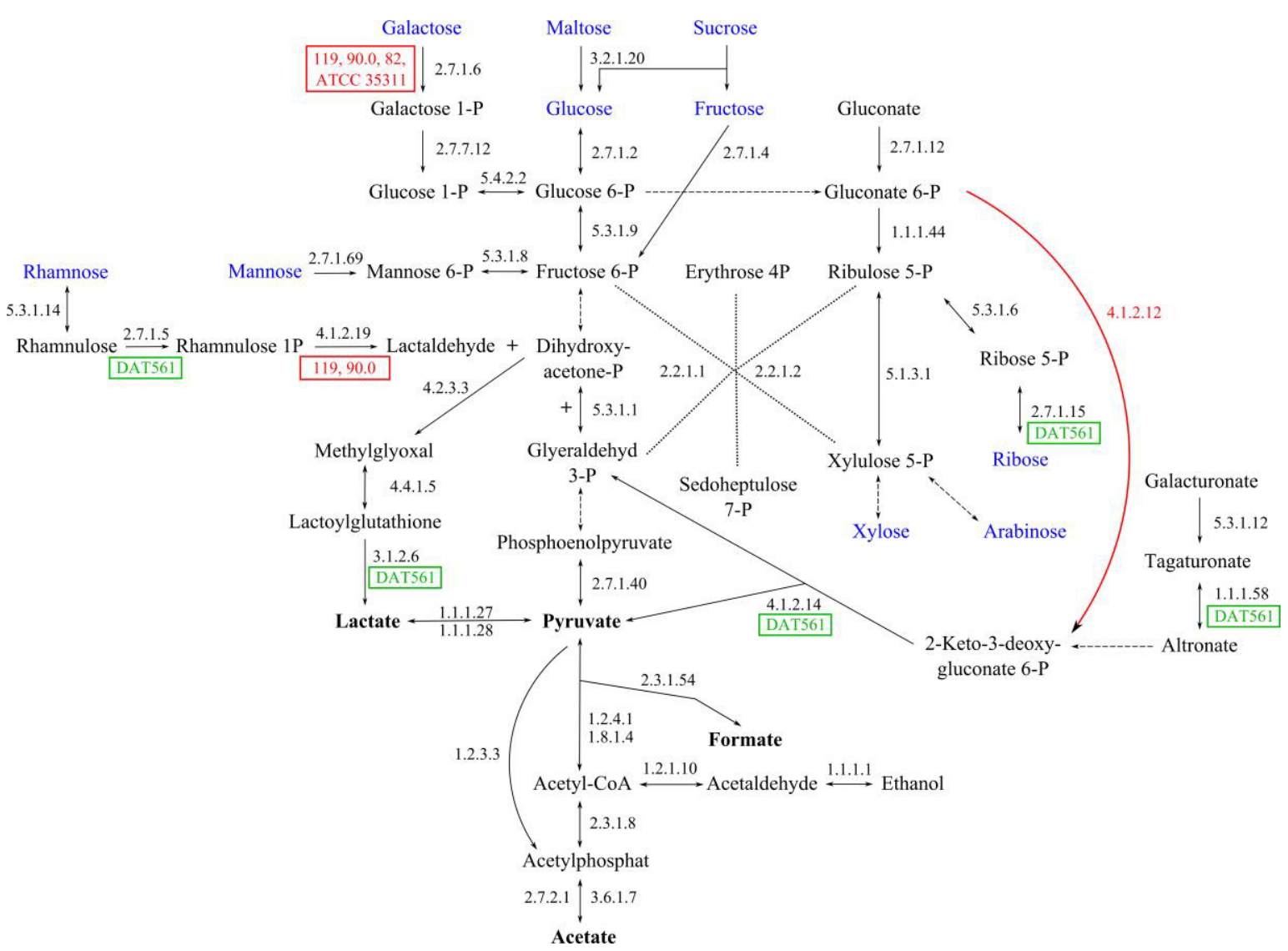

Figure 8: Glycolysis, pentose phosphate pathway, Entner-Doudoroff (ED-) pathway, mixed acid fermentation and sugar interconversions (partly). The reactions are schematized (cofactors, cosubstrates, $\mathrm{CO}_{2}$-formation are not shown). Dotted arrows indicate a summary of multiple reactions, which were found to be present in all strains. Gene products are visualized via EC numbers. Green blocks indicate strain-specific reactions. Red blocks display all strains missing the respective enzyme. Sugars present in honey and degraded pectin backbones (see Supplementary Figure 3) are visualized in blue. Pyruvate and putative end products are shown in bold. All strains lack a pyruvatedecarboxylase (EC 4.1.1.1), which is part of the ethanol fermentation. Additionally, all strains lack a phosphogluconate dehydrogenase (red arrow, EC 4.1.2.12), which is part of the Entner-Doudoroffpathway.

\section{Discussion}

The focus of this study was the genome-based identification of putative virulence factors in the honey bee pathogen M. plutonius and their role in pathogenesis. Based on our findings we predict a model of pathogenesis and infection for M. plutonius (Figure 9) involving the following stages: (i) the uptake of contaminated food and proliferation of M. plutonius in the 
gut of the honey bee larva, (ii) competition for host resources, (iii) breaching the peritrophic matrix, (iv) adhesion to eukaryotic cells and toxin production, and (v) degradation of the honey bee larva and encapsulation of $M$. plutonius.

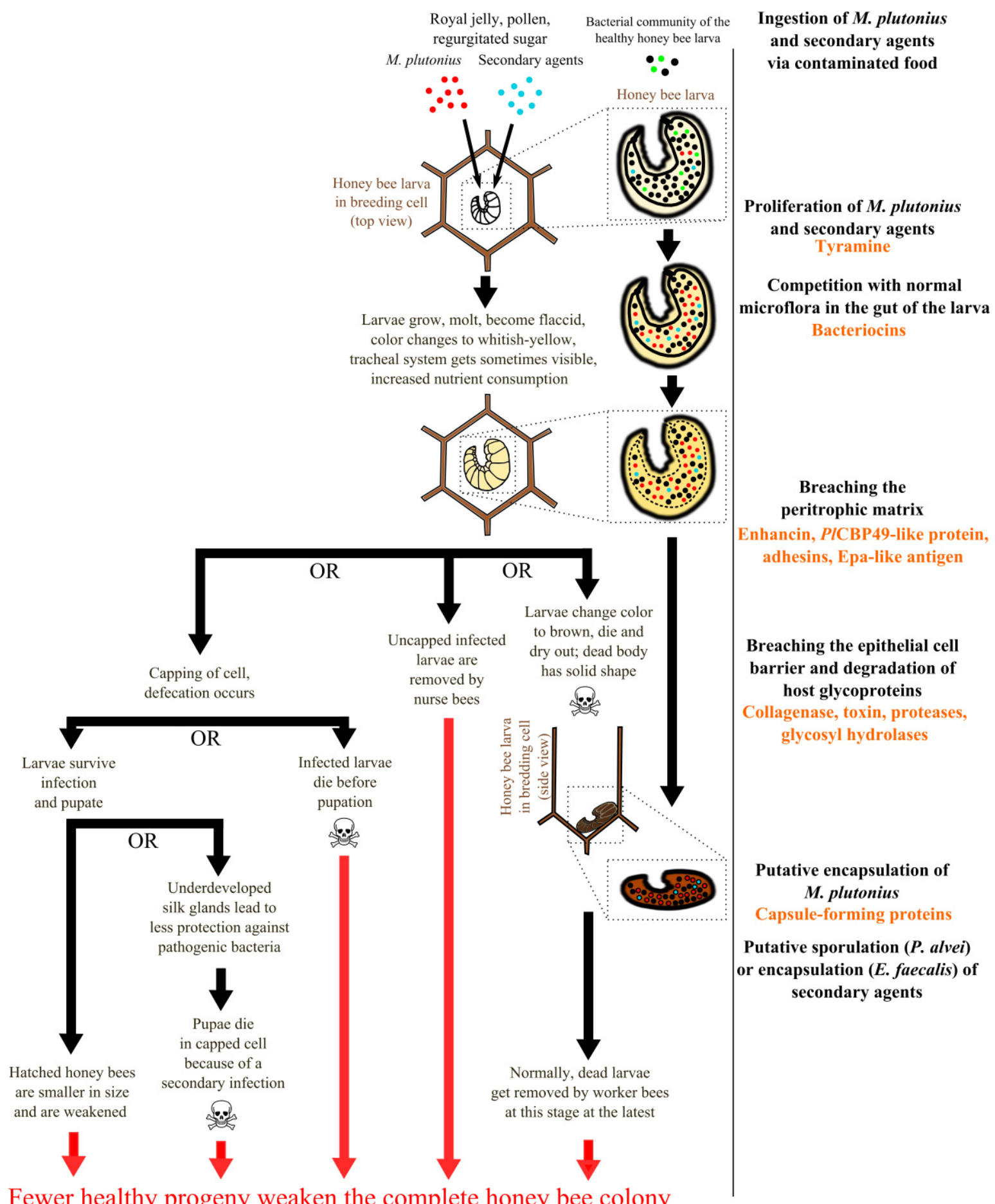

Figure 9: Proposed M. plutonius pathogenesis and infection model. Virulence factors of M. plutonius are shown in orange. 


\section{1. (i) Uptake of contaminated food and proliferation of $M$. plutonius in the gut of} the honey bee larva

The normal development of the healthy honey bee larva lasts 12 days from the egg to pupation. The embryonic development of the honey bee egg lasts three days. Afterwards, nurse bees feed the hatched honey bee larva with royal jelly for another three days. Developing worker larvae are fed with a mixture of glandular secretions produced by nurse bees, honey and pollen for 2.5 to 3.5 days until pupation (Winston, 1987). During the feeding period after the egg hatching, honey bee larvae are subject to infection by M. plutonius via contaminated food (Forsgren, 2010). As only the early larval stages are susceptible to an infection (Bailey and Ball, 1991), royal jelly, honey and beebread represent the major risk of contamination. Although royal jelly possesses antibacterial activity (Fujiwara et al., 1990), bacteria like Lactobacillus kunkeei and the Acetobacteraceae of the "Alpha 2.2" group can survive in this habitat (Corby-Harris et al., 2014). Recently, Asama et al. (2015) detected M. plutonius in royal jelly. Studies about microbes surviving honey were carried out frequently and were based on cloning procedures or cultivation steps (Olaitan et al., 2007; Snowdon and Cliver, 1996). Microorganisms in honey have to withstand its characteristics like concentrated sugar and acidity (Olaitan et al., 2007). M. plutonius was found only twice in honey obtained from EFB-infected honey bee colonies (Wootton et al., 1981; Takamatsu et al., 2014) and in bulked honey (Hornitzky and Smith, 1998). This might be a consequence of methods not sensitive enough for very low bacterial loads and detection of low-abundant bacterial groups. M. plutonius is described as a capsule-forming bacterium (Ritter, 2012) that bears the potential to overcome the antimicrobial effects of honey. Therefore, the presence of encapsulated bacteria in honey is likely. Nevertheless, other food contamination sources have been examined more recently. The detection of M. plutonius in beebread (Anderson et al., 2014; Asama et al., 2015) and the secondary agent E. faecalis in floral nectar (Anderson et al., 2013) indicates a putative pollen- and nectar association of EFB-associated bacteria. The combination of the food sources (glandular secretions, honey, and pollen) and the transfer to honey bee larva habitat may result in optimal growth conditions for several bacteria like L. kunkeei, Alpha- and Gammaproteobacteria, and Clostridia (Vojvodic et al., 2013; Ahn et al., 2012), which reside dormant in encapsulated or sporulated forms.

Our genome analyses revealed that all M. plutonius strains are putatively able to degrade the pectin backbone of the pollen cell wall using a large variety of enzymes. Interestingly, the strains differ in their genetic equipment of these enzymes, as different enzymes are 
putatively non-functional due to mutations in the corresponding genes of all typical strains from Switzerland and Norway (Supplementary Figure 3). Pectin degradation might result in pollen perforation and therefore in the release of its nutrient-rich content (Engel et al., 2012). All strains harbor genes encoding enzymes for the essential energy metabolism pathways glycolysis and the pentose phosphate pathway. The putative lack of function of transketolase and pyruvate kinase of $M$. plutonius DAT 561 is highly questionable and might be a consequence of the 454 sequencing approach chosen by Okumura et al. (2012), which is not suitable to dissolve homopolymer stretches (Luo et al., 2012). As shown in Figure 8 and Supplementary Figure 3, the atypical strain DAT561 is putatively able to use a variety of sugar substrates as energy and carbohydrate sources via glycolysis, pentose phosphate pathway, ED-pathway and sugar interconversions, which supports recent results (Arai et al., 2012). As these substrates are ingredients of honey, royal jelly, and pollen, the atypical strain DAT561 is more adapted to the natural resources found in the larval gut than typical strains, which putatively results in faster growth (Figure 8). Besides the metabolic differences between typical and atypical strains, the production of tyramine by $M$. plutonius might be toxic for honey bee larvae (Kanbar et al., 2004). It was shown that the production of tyramine led to a classic EFB symptom, whereas tyramine-treated larvae changed their color to yellow/brown (Kanbar et al., 2004). In a recent study, Perez et al. (2014) indicated that tyramine biosynthesis is transcriptionally induced at low $\mathrm{pH}$ and improves the fitness of $E$. faecalis in acidic environments. Interestingly, the typical strains 82 (ST32), 90.0 (ST13), 119 (ST20) and ATCC 35311 (ST1) lack the required tyrosine decarboxylase (Figure 4), which could lead to decreased virulence. The aforementioned strains phylogenetically cluster together, while all ST3/ST7 strains form another clade (Figure 3). The assimilation of food and putatively the production of tyramine by $M$. plutonius are the first steps in EFB pathogenesis and impact the further development of the honey bee larva severely. EFB-infected larvae, which survived an EFB infection, are often weakened and spin feeble cocoons due to less well-developed silk glands (Bailey and Ball, 1991). As a consequence, this might favor secondary infections (Figure 9) and the spread of the pathogen to neighboring larvae via the feces (Alippi, 1999).

\section{2. (ii) Competition for host resources}

During the infection cycle it is essential for M. plutonius to be able to compete with the natural microbiota. The M. plutonius genomes contain genetic determinants encoding proteins with high similarity to bacteriocins. Bacteriocins are antimicrobial peptides 
produced by bacteria to impede the growth of competing strains and obtain more nutrients and living space in environments (Yang et al., 2014). The ability to produce bacteriocins in the space-limited and nutrient-embattled environment of the larval gut is an advantage. In the genome of $M$. plutonius, we found three putative genes encoding bacteriocin biosynthesis. These genes only share low amino acid sequence similarity to Zoocin A, a streptococcolytic enzyme (Heath et al., 2004). Interestingly, only the genome of the highly virulent, atypical strain $M$. plutonius DAT561 lacks the respective genes (Figure 4, Supplementary Data 2, Sheet 4). Additionally, only the atypical strain DAT561 and the typical strain M. plutonius ATCC 35311 lack the complete gene cluster encoding an Epa (Figure 4 and Figure 7). Epa was first discovered in E. faecalis (Xu et al., 1997, 1998). Teng et al. (2009) showed that the epa locus is involved in the biosynthesis of a rhamnopolysaccharide. This polysaccharide is important for biofilm formation and virulence in a mouse peritonitis model (Teng et al., 2009; Rigottier-Gois et al., 2014), but also facilitates resistance to antimicrobial peptides. Therefore, Epa might contribute to overall fitness rather than virulence of typical $M$. plutonius strains. We identified three ORFs encoding GH-73 family domain-containing proteins. Usually, GH-73 family proteins are described as beta- $N$-acetylglucosaminidases and were shown to be involved in daughter cell separation (Eckert et al., 2006), although one GH-73 family enzyme of L. monocytogenes acts as a virulence factor during host-cell invasion (Bublitz et al., 2009). Both, typical strains as well as the atypical strain DAT561 harbor such genes but their role in pathogenesis is unknown.

One of the most striking pathogenic features of the AFB pathogen $P$. larvae, the nonribosomal peptide synthases (NRPSs) and polyketide synthases (PKs) (Djukic et al., 2014), are missing in M. plutonius genomes. The NRPS/PKS gene clusters of P. larvae contribute to its antibacterial and antifungal behavior (Sood et al., 2014; Garcia-Gonzalez. et al., 2014a, 2014b). In contrast to $M$. plutonius, pure cultures of $P$. larvae can be obtained from AFB-diseased honey bee larvae, indicating that $P$. larvae is eliminating other bacterial competitors (Garcia-Gonzalez et al., 2014a). The secondary invaders P. alvei, E. faecalis, B. laterosporus and A. eurydice are usually found in the remains of EFB-diseased honey bee larvae. These secondary agents most likely play a role as putative saprophytes (Genersch, 2010; Forsgren, 2010). 


\section{3. (iii) Degradation of larval glycoproteins and breaching the peritrophic matrix}

Virulence of pathogenic organisms is largely determined by the ability to degrade host glycoproteins and to metabolize the resultant carbohydrates. The honey bee larval gut is coated by a chitin-containing peritrophic matrix, which is degraded during $P$. larvae infection (Garcia-Gonzalez and Genersch, 2013). The peritrophic matrix is the first barrier, which has to be overcome when trying to breach the epithelium and enter the haemocoel (Yue et al., 2008; Garcia-Gonzalez et al., 2014c). M. plutonius has been described to be able to destroy the peritrophic matrix (Shimanuki, 1990). A recent study described a new member of the AA10 family of chitin-binding and chitin-degrading lytic polysaccharide monooxygenases, namely PlCBP49, which was identified as a key virulence factor in AFB (Garcia-Gonzalez et al., 2014c). A lack of PlCBP49 activity resulted in approximately 95\% reduction in larval mortality. Interestingly, a PlCBP49 homolog was present in the genomes of the M. plutonius strains. We identified one ORF (Supplementary Data 2, Sheet 4) present in all M. plutonius strains, which shares $37 \%$ identity to PlCBP49 of P. larvae. In addition, we identified one gene in all M. plutonius strains encoding a peptidase M60 family protein (enhancin, PF03272), a metalloprotease that can disrupt the peritrophic matrix (Peng et al., 1999; Tellam et al., 1999; Fang et al., 2009; Toprak et al., 2012) by degrading the insect intestinal mucin (Wang and Granados, 1997). The function of enhancin was originally described for granuloviruses (Wang et al., 1994; Peng et al., 1999), but also for Bacillus and Yersinia species (Galloway et al., 2005; Fang et al., 2009). P. larvae DSM 25719 (genotype ERIC I) and DSM 25430 (genotype ERIC II) contain peptidase M60 family-encoding genes, but these are not functional due to insertion of transposases or frameshift mutations (Djukic et al., 2014) (Figure 5). Both, the PlCBP49-like protein and the putative enhancin could contribute to the virulence of $M$. plutonius.

The peritrophic matrix is comprised of chitin and (glyco)proteins, mainly peritrophins derived from mucins (Terra, 2001). We detected an endo-alpha- $N$-acetylgalactosaminidase (EC 3.2.1.97), which is only encoded by the genomes of the typical ST3/ST7 strains and the atypical strain DAT561 (Figure 3 and Figure 4). Ashida et al. (2008) suggested, that the endo-alpha- $N$-acetylgalactosaminidase of Clostridium perfringens is an extracellular soluble enzyme, which may penetrate the mucin layer and reach the surface of epithelial cells. Therefore, it could be possible that this enzyme is able to damage intestinal mucus (Ashida et al., 2008). The impact of the endo-alpha- $N$-acetylgalactosaminidase of $M$. plutonius on peritrophins of the peritrophic matrix of the epithelial cells still has to be investigated. Like enhancin, the endo-alpha- $N$-acetylgalactosaminidase-encoding gene is transcribed in vivo in 
EFB-infected larvae, but the latter is putatively not functional in four of the genomes (strain 82, 90.0, 119 and ATCC 35311) (Supplementary Data 2, Sheet 4, and Figure 4). According to Yue et al. (2008) and Garcia-Gonzalez and Genersch (2013), the "degradation of the peritrophic matrix is a key step in AFB pathogenesis and might mark the transition from the non-invasive to the invasive stage of infection". Consequently, the degradation of the peritrophic matrix by $M$. plutonius could lead to an invasive stage of infection, too.

\section{4. (iv) Adhesion to eukaryotic cells and toxin production}

Adhesion to extracellular matrix proteins of eukaryotic cells is a key step in virulence of several pathogenic bacteria (Holmes et al., 2001; Massey et al., 2001; Teng et al., 2003; Spigaglia et al., 2013). With putative collagen adhesins, s-layer and cell surface proteins, and a fibronectin/fibrinogen-binding protein, M. plutonius might be able to attach to eukaryotic epithelial cells. Nevertheless, differences within the typical strains and between typical and atypical strains were recorded (Figure 4 and Figure 6). The atypical strain $M$. plutonius DAT561 harbors more putatively functional gene clusters encoding for adhesion and surface proteins, whereas typical strains either lack these clusters or only harbor remnants (Figure 4 and Figure 6). The lack of genes, and the divergences in gene sizes and domain structures of the corresponding proteins, respectively, could contribute to decreased virulence of typical strains. Strikingly, the obtained results also fit to the phylogenetic tree based on a core-genome SNP typing (Figure 3), in which ST3/ST7 strains on the one hand and all other STs on the other hand form different clades.

Regarding pathogenicity, a plasmid (pMP19) was found in M. plutonius 49.3 with highly similar contigs in strains 21.1, 60, and H6. The plasmid comprises 20 ORFs of which two ORFs encoding an extracellular matrix-binding protein and melissotoxin A are putatively contributing to virulence. The extracellular matrix-binding protein shows high similarity to extracellular matrix-binding proteins of diverse Lactobacilli and Streptococci and consists of an N-terminal signal peptide, eight copies of the DUF1542 domain and a C-terminal LPXTG cell wall anchor domain. DUF1542 domains are typically found in cell surface proteins. It has been shown in Staphylococcus aureus that some DUF1542-containing proteins are involved in cellular adhesion and antibiotic resistance (Clarke et al., 2002). The epsilon toxin ETX/mosquitocidal toxin MTX2-like protein (Supplementary Data 2, Sheet 4) encoded by the plasmid is the first toxin identified for M. plutonius, and we could show that it is expressed in vivo (Supplementary Figure 2). Melissotoxin A includes a signal peptide and is most likely extracellular. The related epsilon toxin of Clostridium perfringens is a 
major virulence factor and leads to a rapid change of host cell membrane permeability for ions by forming channels in lipid bilayers (Petit et al., 2001). Interestingly, the virulence plasmid pMP19 is not stably maintained during in vitro propagation, as shown by the absence of the plasmid in M. plutonius $\mathrm{S} 1$, which is a five times laboratory-passaged offspring of M. plutonius 49.3. Furthermore, M. plutonius H6, L9 and B5 were isolated from the same EFB-infected honey bee larva and exhibited a close phylogenetic relationship (Figure 3), but only strain H6 still harbors the plasmid after three cultivation steps. It is already known that typical strains of $M$. plutonius lose their pathogenicity after several cultivation steps in the laboratory (Arai et al., 2012), which is most likely due to the loss of plasmid pMP19. Loss of virulence during in vitro cultivation, as a result of plasmid curing, has already been shown for several important human pathogens such as Shigella flexneri (Schuch and Maurelli, 1997) and Yersinia pestis (Bhaduri and Smith, 2011). We hypothesize that the typical strains 21.1, 49.3, 60 and H6 are more virulent than the other typical strains analyzed in this study because of the presence of the putative virulence plasmid pMP19. Furthermore, these four strains also cluster together in the phylogenetic tree based on the presence/absence of orthologous proteins mainly due to the presence of the plasmid pMP19 (Figure 4). The exact role of the pMP19-encoded melissotoxin A and the extracellular matrix protein have to be investigated further. Nevertheless, other genetic determinants might still be important for virulence, because the atypical strain DAT561 remains virulent even after multiple cultivation steps (Arai et al., 2012), although it lacks pMP19 and genes encoding for melissotoxin A and an extracellular matrix protein. This might be due to a different virulence mechanism of atypical $M$. plutonius strains in comparison to typical strains. A recent study proposed that the genetic variability in the aforementioned epa locus is a key determinant in the ability of E. faecalis to colonize the human gastrointestinal tract due to phase variation (Rigottier-Gois et al., 2014). Thus, the epa locus could play an important role in typical M. plutonius strains, as it putatively mediates biofilm formation (Teng et al., 2009; Rigottier-Gois et al., 2014).

\section{5. (v) Degradation of the honey bee larva and encapsulation of M. plutonius}

Once $M$. plutonius gains access to the larval gut epithelium, proteases possibly in combination with melissotoxin A might be responsible for disruption of the epithelial barrier. We identified 41 different families of proteolytic enzymes encoded by all analyzed $M$. plutonius strains (Supplementary Data 2, Sheet 3a, Sheet 3b). All strains harbor an U32family peptidase, which might serve as a collagenase (Supplementary Data 2, Sheet 4). The 
ability to cleave collagen and collagen fibers with collagenases is regarded as a potent virulence factor concerning invasion and spreading of the bacteria within their host (Harrington, 1996). Additionally, an ORF encoding a membrane-bound serine peptidase (S41A family) was found only in the atypical strain M. plutonius DAT561 (Supplementary Data 2, Sheet 3a, Sheet 3b). However, the role of this protein is unknown, as serine proteases are ubiquitous enzymes with diverse biological functions (Hedstrom, 2002; Barrett, 2004). Once the larva is dead, proteases are further needed for the subsequent degradation of the larval remains. In contrast to AFB (Djukic et al., 2014), secondary agents like P. alvei (Djukic et al., 2012), B. laterosporus (Djukic et al., 2011), L. kunkeei (Djukic et al., 2015), and bacteria of the genus Enterococcus are present in EFB-infected larva. Although $M$. plutonius alone causes EFB, several infectious stages might be enhanced by interplay with these secondary agents, as several of these genera are described as saprophytic (Forsgren, 2010). If nutrients are depleted P. larvae undergoes sporulation. M. plutonius is described to form a capsule that allows survival in feces and wax for several months up to several years (Ritter, 2012). We detected a gene cluster encoding capsule-forming proteins in all $M$. plutonius strains analyzed in this study (Supplementary Data 2, Sheet 4), although all strains contain nonsense mutations in at least one gene putatively needed for its biosynthesis (Figure 4). Nevertheless, capsule-forming strains might be more resistant to disadvantageous environmental changes and able to survive longer in the remains of the diseased larva. As M. plutonius breaches the gut epithelium and enters the hemolymph, it gets into contact with hemocytes and antimicrobial factors of the larva (Evans and Lopez, 2004). Hemocytes respond to infectious particles by phagocytosis and subsequent autolysis (Chan et al., 2009). Various pathogenic bacteria use capsules to resist phagocytosis by host immune cells (Green et al., 1985; Boyce et al., 2000; Neo et al., 2010; Hancock et al., 2012). Thus, expression of a capsule might support virulence in the late stages of the infection.

\subsection{Conclusion and prospects}

With our study, based on the identification of putative virulence genes from different $M$. plutonius genomes, we propose a pathogenesis and infection model (Figure 9) which we believe will be helpful to guide future EFB research. Continuous tyramine secretion, suppression of the natural honey bee larvae flora and consumption of natural resources, breakdown of the peritrophic matrix, putative biofilm formation and adhesion to larval cells, and subsequent toxin production most likely lead to death of the honey bee larvae. The remains putatively are degraded by a set of proteases secreted by $M$. plutonius and secondary 
agents.

The genetic equipment coding for virulence factors differs between most strains (Figure 4). Typical and atypical strains share a PlCBP49-like protein, enhancin, collagenase and cell surface proteins, which putatively represent basic virulence factors needed for infection of the honey bee larva. Moreover, we expect differences in virulence within the typical strains, as the typical strains belonging to CC13 (M. plutonius ATCC 35311 (ST1), 90.0 (ST13), 119 (ST20) and 82 (ST32)) lack putatively important virulence factors (e.g. tyrosine decarboxylase, endo-alpha- $N$-acetylgalactosaminidase) and thus, might be less virulent than ST3/ST7 strains. Additionally, ST3/ST7 strains harboring the virulence plasmid pMP19 might be more virulent than ST3/ST7 strains lacking the plasmid. Additionally, typical and atypical strains putatively established different virulence mechanisms. The highly virulent atypical strain DAT561 lacks a toxin and putatively forms different cell envelope-associated and adhesion-associated proteins. The atypical strain might compensate the missing virulence factors with faster growth in the larval gut by increased metabolic capabilities with respect to usage of different nutrient sources. Faster nutrient consumption of atypical strains might lead to starvation of the honey bee larvae. We assume that in case of atypical strains the combination of fast nutrient consumption and establishment of virulence factors lead to an accelerated death of the honey bee larvae. Nevertheless, infection studies are needed to predict a difference in virulence between atypical strains and typical strains harboring the pMP19-encoded melissotoxin A and the extracellular matrix-binding protein.

\section{Author contributions}

MD, DG and DH performed microbiological and molecular experiments. MD performed in silico genome analysis and drafted the manuscript. MD, DG, JDC, and LG were involved in acquiring genome information. AL and AP supported genome analysis. MD and AP designed microbiological experiments. JDC, LG, and RD supervised the work and were involved in the experimental design. All authors contributed to the writing of the manuscript and approved submission.

\section{Conflict of Interest Statement}

The authors declare that the research was conducted in the absence of any commercial or financial relationships that could be construed as a potential conflict of interest. 


\section{Acknowledgements}

The authors would like to thank Dr. Eva Forsgren for supply of the Norwegian M. plutonius strains and Dr. Heiko Nacke and Dr. Dominik Schneider for proofreading the manuscript. We thank the company Vita Europe (Basingstoke, Great Britain) and especially Wolfgang Egner ( $\mathrm{S}+\mathrm{B}$ medVet GmbH, Babenhausen, Germany) for support with Vita EFB Diagnostic Test kits. Furthermore, we thank the "Bundesministerium für Bildung und Forschung" and the "Niedersächsisches Ministerium für Wissenschaft und Kultur" for support. Additionally, we acknowledge support by the German Research Foundation and the Open Access Publication Funds of the Göttingen University. Financial support was granted by the Swiss Federal Food Safety and Veterinary Office to DG.

\section{References}

Ahn, J.-H., Hong, I.-P., Bok, J.-I., Kim, B.-Y., Song, J., and Weon, H.-Y. (2012). Pyrosequencing analysis of the bacterial communities in the guts of honey bees Apis cerana and Apis mellifera in Korea. J. Microbiol. 50, 735-45. doi:10.1007/s12275-0122188-0.

Aizen, M. A., Garibaldi, L. A., Cunningham, S. A., and Klein, A. M. (2008). Long-term global trends in crop yield and production reveal no current pollination shortage but increasing pollinator dependency. Curr. Biol. 18, 1572-5. doi:10.1016/j.cub.2008.08.066.

Aizen, M. A., and Harder, L. D. (2009). The global stock of domesticated honey bees is growing slower than agricultural demand for pollination. Curr. Biol. 19, 915-8. doi:10.1016/j.cub.2009.03.071.

Alikhan, N.-F., Petty, N. K., Ben Zakour, N. L., and Beatson, S. A. (2011). BLAST Ring Image Generator (BRIG): simple prokaryote genome comparisons. BMC Genomics 12, 402. doi:10.1186/1471-2164-12-402.

Alippi, A. (1999). "Bacterial diseases," in Bee disease diagnosis, eds. M. Colin, B. Ball, and M. Kilani (Zaragoza: CIHEAM), 31-59. 
Anderson, K. E., Carroll, M. J., Sheehan, T., Mott, B. M., Maes, P., and Corby-Harris, V. (2014). Hive-stored pollen of honey bees: Many lines of evidence are consistent with pollen preservation, not nutrient conversion. Mol. Ecol. doi:10.1111/mec.12966.

Anderson, K. E., Sheehan, T. H., Mott, B. M., Maes, P., Snyder, L., Schwan, M. R., et al. (2013). Microbial ecology of the hive and pollination landscape: bacterial associates from floral nectar, the alimentary tract and stored food of honey bees (Apis mellifera). PLoS One 8, e83125. doi:10.1371/journal.pone.0083125.

Arai, R., Tominaga, K., Wu, M., Okura, M., Ito, K., Okamura, N., et al. (2012). Diversity of Melissococcus plutonius from Honeybee Larvae in Japan and Experimental Reproduction of European Foulbrood with Cultured Atypical Isolates. PLoS One 7, e33708. doi:10.1371/journal.pone.0033708.

Asama, T., Arima, T.-H., Gomi, T., Keishi, T., Tani, H., Kimura, Y., et al. (2015). Lactobacillus kunkeei YB38 from honeybee products enhances IgA production in healthy adults. J. Appl. Microbiol., in press. doi:10.1111/jam.12889.

Ashida, H., Maki, R., Ozawa, H., Tani, Y., Kiyohara, M., Fujita, M., et al. (2008). Characterization of two different endo-alpha- $N$-acetylgalactosaminidases from probiotic and pathogenic enterobacteria, Bifidobacterium longum and Clostridium perfringens. Glycobiology 18, 727-34. doi:10.1093/glycob/cwn053.

Bailey, L. (1957a). European Foul Brood: a Disease of the Larval Honeybee (Apis mellifera L.) caused by a Combination of Streptococcus pluton (Bacillus pluton White) and Bacterium eurydice White. Nature 180, 1214-1215.

Bailey, L. (1957b). The Isolation and Cultural Characteristics of Streptococcus pluton and further Oberservations on Bacterium eurydice. J. gen. Microbiol. 17, 39-48.

Bailey, L., and Ball, B. (1991). Honey Bee Pathology. London: Academic Press Limited.

Bailey, L., and Collins, M. D. (1982). Reclassification of "Streptococcus pluton" (White) in a new genus Melissococcus, as Melissococcus pluton nom. rev.; comb. nov. J. Appl. Bacteriol. 53, 215-217. doi:10.1111/j.1365-2672.1982.tb04679.x. 
Bankevich, A., Nurk, S., Antipov, D., Gurevich, A. A., Dvorkin, M., Kulikov, A. S., et al. (2012). SPAdes: a new genome assembly algorithm and its applications to single-cell sequencing. J. Comput. Biol. 19, 455-77. doi:10.1089/cmb.2012.0021.

Barrett, A. J. (2004). Bioinformatics of proteases in the MEROPS database. Curr. Opin. Drug Discov. Devel. 7, 334-41.

Bhaduri, S., and Smith, J. L. (2011). Virulence Plasmid (pYV)-Associated Expression of Phenotypic Virulent Determinants in Pathogenic Yersinia Species: A Convenient Method for Monitoring the Presence of pYV under Culture Conditions and Its Application for Isolation/Detection of Yersinia pestis in Food. J. Pathog. 2011, 727313. doi:10.4061/2011/727313.

Boyce, J. D., Chung, J. Y., and Adler, B. (2000). Pasteurella multocida capsule: Composition, function and genetics. J. Biotechnol. 83, 153-160. doi:10.1016/S01681656(00)00309-6.

Bublitz, M., Polle, L., Holland, C., Heinz, D. W., Nimtz, M., and Schubert, W. D. (2009). Structural basis for autoinhibition and activation of Auto, a virulence-associated peptidoglycan hydrolase of Listeria monocytogenes. Mol. Microbiol. 71, 1509-1522. doi:10.1111/j.1365-2958.2009.06619.x.

Campos, M. A., Vargas, M. A., Regueiro, V., Llompart, C. M., Albertí, S., and Bengoechea, J. A. (2004). Capsule Polysaccharide Mediates Bacterial Resistance to Antimicrobial Peptides. Infect. Immun 72, 7107-7114. doi:10.1128/IAI.72.12.7107.

Chan, Q. W. T., Melathopoulos, A. P., Pernal, S. F., and Foster, L. J. (2009). The innate immune and systemic response in honey bees to a bacterial pathogen, Paenibacillus larvae. BMC Genomics 10, 9. doi:387 10.1186/1471-2164-10-387.

Chen, L., Xiong, Z., Sun, L., Yang, J., and Jin, Q. (2012). VFDB 2012 update: toward the genetic diversity and molecular evolution of bacterial virulence factors. Nucleic Acids Res. 40, D641-5. doi:10.1093/nar/gkr989.

Chevreux, B., Wetter, T., and Suhai, S. (1999). Genome sequence assembly using trace signals and additional sequence information. Comput. Sci. Biol.: Proc. German Conference on Bioinformatics GCB '99 GCB, 45-56. 
Clarke, S. R., Harris, L. G., Richards, R. G., and Foster, S. J. (2002). Analysis of Ebh, a 1.1megadalton cell wall-associated fibronectin-binding protein of Staphylococcus aureus. Infect. Immun. 70, 6680-7. doi:10.1128/IAI.70.12.6680-6687.2002.

Connil, N., Breton, Y. L., Dousset, X., Auffray, Y., Rince, A., and Prévost, H. (2002). Identification of the Enterococcus faecalis Tyrosine Decarboxylase Operon Involved in Tyramine Production. 68, 3537-3544. doi:10.1128/AEM.68.7.3537.

Corby-Harris, V., Maes, P., and Anderson, K. E. (2014). The Bacterial Communities Associated with Honey Bee (Apis mellifera) Foragers. PLoS One 9, e95056. doi:10.1371/journal.pone.0095056.

Courtney, H. S., Li, Y., Dale, J. B., and Hasty, D. L. (1994). Cloning, sequencing, and expression of a fibronectin/fibrinogen-binding protein from group A streptococci. Infect. Immun. 62, 3937-46.

Djukic, M., Becker, D., Poehlein, A., Voget, S., and Daniel, R. (2012). Genome sequence of Paenibacillus alvei DSM 29, a secondary invader during European foulbrood outbreaks. J. Bacteriol. 194, 6365. doi:10.1128/JB.01698-12.

Djukic, M., Brzuszkiewicz, E., Fünfhaus, A., Voss, J., Gollnow, K., Poppinga, L., et al. (2014). How to Kill the Honey Bee Larva: Genomic Potential and Virulence Mechanisms of Paenibacillus larvae. PLoS One 9, e90914. doi:10.1371/journal.pone.0090914.

Djukic, M., Poehlein, A., Strauß, J., Tann, F., Leimbach, A., Hoppert, M., et al. (2015). High quality draft genome of Lactobacillus kunkeei EFB6, isolated from a German European foulbrood outbreak of honeybees. Stand. Genomic Sci. 10, 16. doi:10.1186/1944-3277$10-16$.

Djukic, M., Poehlein, A., Thürmer, A., and Daniel, R. (2011). Genome sequence of Brevibacillus laterosporus LMG 15441, a pathogen of invertebrates. J. Bacteriol. 193, 5535-6. doi:10.1128/JB.05696-11.

Dramsi, S., Bourdichon, F., Cabanes, D., Lecuit, M., Fsihi, H., and Cossart, P. (2004). FbpA, a novel multifunctional Listeria monocytogenes virulence factor. Mol. Microbiol. 53, 639-649. doi:10.1111/j.1365-2958.2004.04138.x. 
Eckert, C., Lecerf, M., Dubost, L., Arthur, M., and Mesnage, S. (2006). Functional analysis of AtlA, the major $\mathrm{N}$-acetylglucosaminidase of Enterococcus faecalis. J. Bacteriol. 188, 8513-8519. doi:10.1128/JB.01145-06.

Engel, P., Martinson, V. G., and Moran, N. A. (2012). Functional diversity within the simple gut microbiota of the honey bee. Proc. Natl. Acad. Sci. U. S. A. 109, 11002-7. doi:10.1073/pnas.1202970109.

Evans, J. D., and Lopez, D. L. (2004). Bacterial Probiotics Induce an Immune Response in the Honey Bee (Hymenoptera: Apidae). 97, 752-756. doi:http://dx.doi.org/10.1603/0022-0493(2004)097[0752:BPIAIR]2.0.CO;2.

Fang, S., Wang, L., Guo, W., Zhang, X., Peng, D., Luo, C., et al. (2009). Bacillus thuringiensis bel protein enhances the toxicity of Cry1Ac protein to Helicoverpa armigera larvae by degrading insect intestinal mucin. Appl. Environ. Microbiol. 75, 5237-43. doi:10.1128/AEM.00532-09.

Forsgren, E. (2010). European foulbrood in honey bees. J. Invertebr. Pathol. 103 Suppl, S5-9. doi:10.1016/j.jip.2009.06.016.

Francisco, A. P., Vaz, C., Monteiro, P. T., Melo-Cristino, J., Ramirez, M., and Carrio, J. A. (2012). PHYLOViZ: phylogenetic inference and data visualization for sequence based typing methods. BMC Bioinformatics 13, 87. doi:10.1186/1471-2105-13-87.

Fujiwara, S., Imai, J., Fujiwara, M., Yaeshima, T., Kawashima, T., and Kobayashi, K. (1990). A potent antibacterial protein in royal jelly. Purification and determination of the primary structure of royalisin. J. Biol. Chem. 265, 11333-7.

Gallai, N., Salles, J.-M., Settele, J., and Vaissière, B. E. (2009). Economic valuation of the vulnerability of world agriculture confronted with pollinator decline. Ecol. Econ. 68, 810-821. doi:10.1016/j.ecolecon.2008.06.014.

Galloway, C. S., Wang, P., Winstanley, D., and Jones, I. M. (2005). Comparison of the bacterial Enhancin-like proteins from Yersinia and Bacillus spp. with a baculovirus Enhancin. J. Invertebr. Pathol. 90, 134-137. doi:10.1016/j.jip.2005.06.008. 
Garcia-Gonzalez, E., and Genersch, E. (2013). Honey bee larval peritrophic matrix degradation during infection with Paenibacillus larvae, the aetiological agent of American foulbrood of honey bees, is a key step in pathogenesis. Environ. Microbiol. 15, 2894-2901. doi:10.1111/1462-2920.12167.

Garcia-Gonzalez, E., Müller, S., Ensle, P., Süssmuth, R. D., and Genersch, E. (2014a). Elucidation of sevadicin, a novel non-ribosomal peptide secondary metabolite produced by the honey bee pathogenic bacterium Paenibacillus larvae. Environ. Microbiol. 16, 1297-309. doi:10.1111/1462-2920.12417.

Garcia-Gonzalez, E., Müller, S., Hertlein, G., Heid, N., Süssmuth, R. D., and Genersch, E. (2014b). Biological effects of paenilamicin, a secondary metabolite antibiotic produced by the honey bee pathogenic bacterium Paenibacillus larvae. Microbiologyopen 3, 642656. doi:10.1002/mbo3.195.

Garcia-Gonzalez, E., Poppinga, L., Fünfhaus, A., Hertlein, G., Hedtke, K., Jakubowska, A., et al. (2014c). Paenibacillus larvae chitin-degrading protein PICBP49 is a key virulence factor in American Foulbrood of honey bees. PLoS Pathog. 10, e1004284. doi:10.1371/journal.ppat.1004284.

Gasteiger, E., Jung, E., and Bairoch, A. (2001). SWISS-PROT: connecting biomolecular knowledge via a protein database. Curr. Issues Mol. Biol. 3, 47-55.

Genersch, E. (2010). Honey bee pathology: current threats to honey bees and beekeeping. Appl. Microbiol. Biotechnol. 87, 87-97. doi:10.1007/s00253-010-2573-8.

Govan, V. A., Brözel, V., Allsopp, M. H., and Davison, S. (1998). A PCR detection method for rapid identification of Melissococcus pluton in honeybee larvae. Appl. Environ. Microbiol. 64, 1983-5.

Green, B. D., Battisti, L., Koehler, T. M., Thorne, C. B., and Ivins, B. E. (1985). Demonstration of a capsule plasmid in Bacillus anthracis. Infect. Immun. 49, 291-297.

Hammami, R., Zouhir, A., Le Lay, C., Ben Hamida, J., and Fliss, I. (2010). BACTIBASE second release: a database and tool platform for bacteriocin characterization. BMC Microbiol. 10, 22. doi:10.1186/1471-2180-10-22. 
Hancock, L. E., Murray, B. E., and Sillanpää, J. (2012). "Enterococcal Cell Wall Components and Structures," in Enterococci: From Commensals to Leading Causes of Drug Resistant Infection, eds. M. Gilmore, D. Clewell, Y. Ike, and N. Shankar (Boston, MA: Massachusetts Eye and Ear Infirmary).

Harrington, D. J. (1996). Bacterial collagenases and collagen-degrading enzymes and their potential role in human disease. Infect. Immun. 64, 1885-1891.

Haynes, E., Helgason, T., Young, J. P. W., Thwaites, R., and Budge, G. E. (2013). A typing scheme for the honeybee pathogen Melissococcus plutonius allows detection of disease transmission events and a study of the distribution of variants. 5, 525-529. doi:10.1111/1758-2229.12057.

Heath, L. S., Heath, H. E., LeBlanc, P. A., Smithberg, S. R., Dufour, M., Simmonds, R. S., et al. (2004). The streptococcolytic enzyme zoocin A is a penicillin-binding protein. FEMS Microbiol. Lett. 236, 205-211. doi:10.1016/j.femsle.2004.05.038.

Hedstrom, L. (2002). Serine protease mechanism and specificity. Chem. Rev. 102, 4501-24.

Van Heel, A. J., de Jong, A., Montalbán-López, M., Kok, J., and Kuipers, O. P. (2013). BAGEL3: Automated identification of genes encoding bacteriocins and (non)bactericidal posttranslationally modified peptides. Nucleic Acids Res. 41, 448-453. doi:10.1093/nar/gkt391.

Holmes, A. R., McNab, R., Millsap, K. W., Rohde, M., Hammerschmidt, S., Mawdsley, J. L., et al. (2001). The pavA gene of Streptococcus pneumoniae encodes a fibronectinbinding protein that is essential for virulence. Mol. Microbiol. 41, 1395-408.

Hornitzky, M. A., and Smith, L. (1998). Procedures For The Culture Of Melissococcus Pluton From Diseased Brood And Bulk Honey Samples. J. Apic. Res. 37, 293-294.

Huson, D. H., and Scornavacca, C. (2012). Dendroscope 3: An interactive tool for rooted phylogenetic trees and networks. Syst. Biol. 61, 1061-1067. doi:10.1093/sysbio/sys062. 
Jones, P., Binns, D., Chang, H.-Y., Fraser, M., Li, W., McAnulla, C., et al. (2014). InterProScan 5: genome-scale protein function classification. Bioinformatics 30, 1236 40. doi:10.1093/bioinformatics/btu031.

Joris, B., Englebert, S., Chu, C. P., Kariyama, R., Daneo-Moore, L., Shockman, G. D., et al. (1992). Modular design of the Enterococcus hirae muramidase-2 and Streptococcus faecalis autolysin. FEMS Microbiol. Lett. 70, 257-264. doi:10.1016/03781097(92)90707-u.

Kanbar, G., Engels, W., Nicholson, G., Hertle, R., and Winkelmann, G. (2005). Corrigendum to: Tyramine functions as a toxin in honey bee larvae during -transmitted infection by Melissococcus plutonius [FEMS Microbiol. Lett. 234 (2004) 149-154]. FEMS Microbiol. Lett. 245, 193-193. doi:10.1016/j.femsle.2004.03.022.

Kanbar, G., Engels, W., Nicholson, G. J., Hertle, R., and Winkelmann, G. (2004). Tyramine functions as a toxin in honey bee larvae during Varroa-transmitted infection by Melissococcus pluton. FEMS Microbiol. Lett. 234, 149-54. doi:10.1016/j.femsle.2004.03.022.

Lagesen, K., Hallin, P., Rødland, E. A., Staerfeldt, H.-H., Rognes, T., and Ussery, D. W. (2007). RNAmmer: consistent and rapid annotation of ribosomal RNA genes. Nucleic Acids Res. 35, 3100-8. doi:10.1093/nar/gkm160.

Larsen, M. V., Cosentino, S., Rasmussen, S., Friis, C., Hasman, H., Marvig, R. L., et al. (2012). Multilocus sequence typing of total-genome-sequenced bacteria. J. Clin. Microbiol. 50, 1355-1361. doi:10.1128/JCM.06094-11.

Lechner, M., Findeiss, S., Steiner, L., Marz, M., Stadler, P. F., and Prohaska, S. J. (2011). Proteinortho: detection of (co-)orthologs in large-scale analysis. BMC Bioinformatics 12, 124. doi:10.1186/1471-2105-12-124.

Leimbach, A. bac-genomics-scripts. 2014. Available at: https://github.com/aleimba/bacgenomics-scripts.

Lowe, T. M., and Eddy, S. R. (1997). tRNAscan-SE: a program for improved detection of transfer RNA genes in genomic sequence. Nucleic Acids Res. 25, 955-64. 
Luo, C., Tsementzi, D., Kyrpides, N., Read, T., and Konstantinidis, K. T. (2012). Direct comparisons of Illumina vs. Roche 454 sequencing technologies on the same microbial community DNA sample. PLoS One 7. doi:10.1371/journal.pone.0030087.

Markowitz, V. M., Mavromatis, K., Ivanova, N. N., Chen, I.-M. A., Chu, K., and Kyrpides, N. C. (2009). IMG ER: a system for microbial genome annotation expert review and curation. Bioinformatics 25, 2271-8. doi:10.1093/bioinformatics/btp393.

Massey, R. C., Kantzanou, M. N., Fowler, T., Day, N. P. J., Schofield, K., Elisabeth, R., et al. (2001). Fibronectin-binding protein A of Staphylococcus aureus has multiple, substituting, binding regions that mediate adherence to fibronectin and invasion of endothelial cells. Cell Microbiol. 3, 839-851.

Morse R. A., and Calderone, N. W. (2000). The value of honey bees as pollinators of U.S. crops in 2000. Bee Cult. 128, 1-15.

Neo, Y., Li, R., Howe, J., Hoo, R., Pant, A., Ho, S., et al. (2010). Evidence for an intact polysaccharide capsule in Bordetella pertussis. Microbes Infect. 12, 238-245. doi:10.1016/j.micinf.2009.12.002.

O’Donovan, C., Martin, M. J., Gattiker, A., Gasteiger, E., Bairoch, A., and Apweiler, R. (2002). High-quality protein knowledge resource: SWISS-PROT and TrEMBL. Brief. Bioinform. 3, 275-84.

Okumura, K., Arai, R., Okura, M., Kirikae, T., Takamatsu, D., Osaki, M., et al. (2011). Complete genome sequence of Melissococcus plutonius ATCC 35311. J. Bacteriol. 193, 4029-30. doi:10.1128/JB.05151-11.

Okumura, K., Arai, R., Okura, M., Kirikae, T., Takamatsu, D., Osaki, M., et al. (2012). Complete genome sequence of Melissococcus plutonius DAT561, a strain that shows an unusual growth profile and is representative of an endemic cluster in Japan. $J$. Bacteriol. 194, 3014. doi:10.1128/JB.00437-12.

Olaitan, P. B., Adeleke, O. E., and Ola, I. O. (2007). Honey: A reservoir for microorganisms and an inhibitory agent for microbes. Afr. Health Sci. 7, 159-165. doi:10.5555/afhs.2007.7.3.159. 
Palmer, K. L., Godfrey, P., Griggs, A., Kos, V. N., Zucker, J., Desjardins, C., et al. (2012). Comparative Genomics of Enterococci: Variation in Enterococcus faecalis, Clade Structure in E. faecium, and Defining Characteristics of E. gallinarum and E. casseliflavus. MBio 3, e00318-11-e00318-11. doi:10.1128/mBio.00318-11.

Peng, J., Zhong, J., and R. Granados, R. (1999). A baculovirus enhancin alters the permeability of a mucosal midgut peritrophic matrix from lepidopteran larvae. J. Insect Physiol. 45, 159-166. doi:10.1016/S0022-1910(98)00110-3.

Perez, M., Calles-Enríquez, M., Nes, I., Martin, M. C., Fernandez, M., Ladero, V., et al. (2015). Tyramine biosynthesis is transcriptionally induced at low $\mathrm{pH}$ and improves the fitness of Enterococcus faecalis in acidic environments. Appl. Microbiol. Biotechnol. 99, 3547-58. doi:10.1007/s00253-014-6301-7.

Petit, L., Maier, E., Gibert, M., Popoff, M. R., and Benz, R. (2001). Clostridium perfringens epsilon toxin induces a rapid change of cell membrane permeability to ions and forms channels in artificial lipid bilayers. J. Biol. Chem. 276, 15736-40. doi:10.1074/jbc.M010412200.

Poppinga, L., and Genersch, E. (2015). Molecular pathogenesis of American Foulbrood: how Paenibacillus larvae kills honey bee larvae. Curr. Opin. Insect Sci., 1-8. doi:10.1016/j.cois.2015.04.013.

Rawlings, N. D., Waller, M., Barrett, A. J., and Bateman, A. (2014). MEROPS: the database of proteolytic enzymes, their substrates and inhibitors. Nucleic Acids Res. 42, D503-9. doi:10.1093/nar/gkt953.

Rigottier-Gois, L., Madec, C., Navickas, A., Matos, R. C., Akary-Lepage, E., Mistou, M.Y., et al. (2014). The Surface Rhamnopolysaccharide Epa of Enterococcus faecalis Is a Key Determinant of Intestinal Colonization. J. Infect. Dis. 211, 62-71. doi:10.1093/infdis/jiu402.

Ritter, W. (2012). Bienen gesund erhalten. Stuttgart: Eugen Ulmer.

Schembri, M. A., Dalsgaard, D., and Klemm, P. (2004). Capsule Shields the Function of Short Bacterial Adhesins. J. Bacteriol. 186, 1249-1257. doi:10.1128/JB.186.5.12491257.2004. 
Schroeder, K., Jularic, M., Horsburgh, S. M., Hirschhausen, N., Neumann, C., Bertling, A., et al. (2009). Molecular characterization of a novel Staphylococcus aureus surface protein (SasC) involved in cell aggregation and biofilm accumulation. PLoS One 4. doi:10.1371/journal.pone.0007567.

Schuch, R., and Maurelli, A. T. (1997). Virulence plasmid instability in Shigella flexneri 2a is induced by virulence gene expression. Infect. Immun. 65, 3686-3692.

Seemann, T. (2014). Prokka: rapid prokaryotic genome annotation. Bioinformatics 30, 2068-9. doi:10.1093/bioinformatics/btu153.

Shimanuki, H. (1990). "Bacteria," in Honey Bee Pests, Predators, and Diseases, eds. R. Morse and R. Nowogrodzki (New York: Cornell University Press), 27-47.

Snowdon, J. A., and Cliver, D. O. (1996). Microorganisms in honey. Int. J. Food Microbiol. 31, 1-26. doi:10.1016/0168-1605(96)00970-1.

Sood, S., Steinmetz, H., Beims, H., Mohr, K. I., Stadler, M., Djukic, M., et al. (2014). Paenilarvins: Iturin Family Lipopeptides from the Honey Bee Pathogen Paenibacillus larvae. ChemBioChem, 15, 1947-1955. doi:10.1002/cbic.201402139.

Spigaglia, P., Barketi-Klai, A., Collignon, A., Mastrantonio, P., Barbanti, F., Rupnik, M., et al. (2013). Surface-layer (S-layer) of human and animal Clostridium difficile strains and their behaviour in adherence to epithelial cells and intestinal colonization. J. Med. Microbiol. 62, 1386-93. doi:10.1099/jmm.0.056556-0.

Staden, R., Beal, K. F., and Bonfield, J. K. (2000). The Staden package, 1998. Methods Mol Biol 132, 115-130.

Stamatakis, A. (2014). RAxML version 8: a tool for phylogenetic analysis and post-analysis of large phylogenies. Bioinformatics 30, 1312-3. doi:10.1093/bioinformatics/btu033.

Sullivan, M. J., Petty, N. K., and Beatson, S. A. (2011). Easyfig: a genome comparison visualizer. Bioinformatics 27, 1009-10. doi:10.1093/bioinformatics/btr039.

Takamatsu, D., Morinishi, K., Arai, R., Sakamoto, A., Okura, M., and Osaki, M. (2014). Typing of Melissococcus plutonius isolated from European and Japanese honeybees 
suggests spread of sequence types across borders and between different Apis species. Vet. Microbiol. 171, 221-6. doi:10.1016/j.vetmic.2014.03.036.

Tellam, R. L., Wijffels, G., and Willadsen, P. (1999). Peritrophic matrix proteins. Insect Biochem. Mol. Biol. 29, 87-101.

Teng, F., Kawalec, M., Weinstock, G. M., Hryniewicz, W., and Murray, B. E. (2003). An Enterococcus faecium secreted antigen, SagA, exhibits broad-spectrum binding to extracellular matrix proteins and appears essential for E. faecium growth. Infect. Immun. 71, 5033-41. doi:10.1128/IAI.71.9.5033.

Teng, F., Singh, K. V., Bourgogne, A., Zeng, J., and Murray, B. E. (2009). Further characterization of the epa gene cluster and Epa polysaccharides of Enterococcus faecalis. Infect. Immun. 77, 3759-3767. doi:10.1128/IAI.00149-09.

Terra, W. R. (2001). The origin and functions of the insect peritrophic membrane and peritrophic gel. Arch. Insect Biochem. Physiol. 47, 47-61. doi:10.1002/arch.1036.

Toprak, U., Harris, S., Baldwin, D., Theilmann, D., Gillott, C., Hegedus, D. D., et al. (2012). Role of enhancin in Mamestra configurata nucleopolyhedrovirus virulence: selective degradation of host peritrophic matrix proteins. J. Gen. Virol. 93, 744-53. doi:10.1099/vir.0.038117-0.

Treangen, T. J., Ondov, B. D., Koren, S., and Phillippy, A. M. (2014). The Harvest suite for rapid core-genome alignment and visualization of thousands of intraspecific microbial genomes. Genome Biol. 15, 524. doi:10.1186/s13059-014-0524-x.

Tutar, Y. (2012). Pseudogenes. Comp. Funct. Genomics 2012. doi:10.1155/2012/424526.

Vojvodic, S., Rehan, S. M., and Anderson, K. E. (2013). Microbial gut diversity of Africanized and European honey bee larval instars. PLoS One 8, e72106. doi:10.1371/journal.pone.0072106.

Wang, P., and Granados, R. R. (1997). An intestinal mucin is the target substrate for a baculovirus enhancin. Proc. Natl. Acad. Sci. U. S. A. 94, 6977-82.

Wang, P., Hammer, D. A., and Granados, R. R. (1994). Interaction of Trichoplusia ni granulosis virus-encoded enhancin with the midgut epithelium and peritrophic 
membrane of four lepidopteran insects. J. Gen. Virol. 75, 1961-1967. doi:10.1099/0022-1317-75-8-1961.

Williams, D. L. (2000). A veterinary approach to the European honey bee (Apis mellifera). Vet. J. 160, 61-73. doi:10.1053/tvj1.2000.0474.

Winston, M. L. (1987). The Biology of the Honey Bee. London: Havard University Press.

Wootton, M., Hornitzky, M. A. Z., and Ryland, L. (1981). Thermal destruction of Streptococcus pluton in Australian honeys and its effect on honey quality. J. Apic. Res. 20, 115-120.

Xu, Y., Jiang, L., Murray, B. E., and Weinstock, G. M. (1997). Enterococcus faecalis antigens in human infections. Infect. Immun. 65, 4207-4215.

Xu, Y., Murray, B. E., and Weinstock, G. M. (1998). A cluster of genes involved in polysaccharide biosynthesis from Enterococcus faecalis OG1RF. Infect. Immun. 66, $4313-4323$.

Yang, S.-C., Lin, C.-H., Sung, C. T., and Fang, J.-Y. (2014). Antibacterial activities of bacteriocins: application in foods and pharmaceuticals. Front. Microbiol. 5, 241. doi:10.3389/fmicb.2014.00241.

Yue, D., Nordhoff, M., Wieler, L. H., and Genersch, E. (2008). Fluorescence in situ hybridization (FISH) analysis of the interactions between honeybee larvae and Paenibacillus larvae, the causative agent of American foulbrood of honeybees (Apis mellifera). Environ. Microbiol. 10, 1612-20. doi:10.1111/j.1462-2920.2008.01579.x.

Zacharof, M. P., and Lovitt, R. W. (2012). Bacteriocins Produced by Lactic Acid Bacteria a Review Article. APCBEE Procedia 2, 50-56. doi:10.1016/j.apcbee.2012.06.010.

Zhou, Y., Liang, Y., Lynch, K. H., Dennis, J. J., and Wishart, D. S. (2011). PHAST: a fast phage search tool. Nucleic Acids Res. 39, W347-52. doi:10.1093/nar/gkr485. 


\title{
4.1 Ergänzende Daten zu Kapitel B4
}

\author{
Inhaltsverzeichnis
}

Supplementary Data 1. Genbank files of M. plutonius ATCC 35311 and DAT561.

Supplementary Data 2. Detection of putative virulence factors.

"Supplementary Data" 1 und 2 sind auf dem beigefügten, digitalen Medium verfügbar. Dazu bitte den Ordner „Kapitel_B4_Ergänzende_Daten“ öffnen.

Supplementary Figure 1. Comparison of bacteriocin biosynthesis and transport clusters of M. plutonius S1 with clusters of M. plutonius DAT561, Enterococcus faecalis FLY1 (accession nr. NZ_ACAR00000000) and Streptococcus iniae ISNO (accession nr. CP007587).

Supplementary Figure 2. Expression of $M$. plutonius putative virulence factors during infection.

Supplementary Figure 3. Pectin degradation by $M$. plutonius. Adaptation of Figure 3A from Engel et al. (2012).

Supplementary Table 1. Primer used in this study.

Supplementary Table 2. General data about M. plutonius strains used in this study. 
$\mathbf{A}$

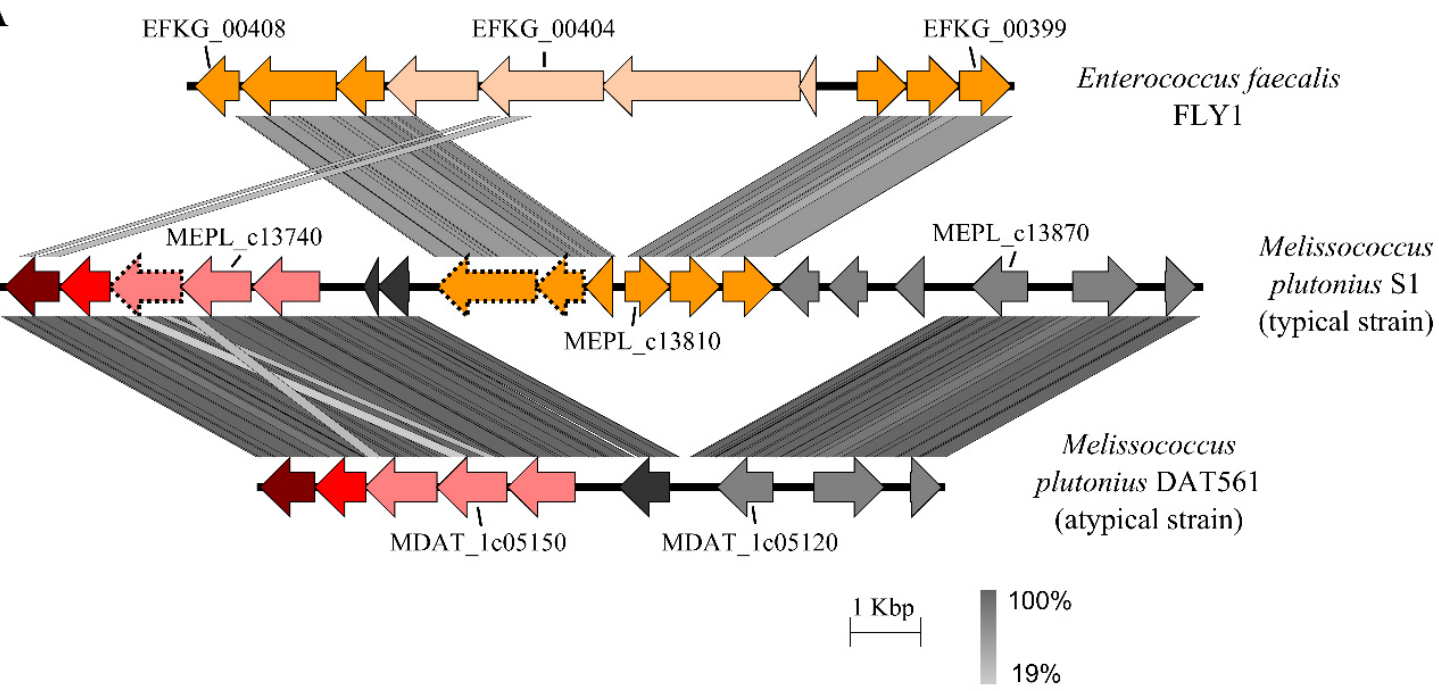

B

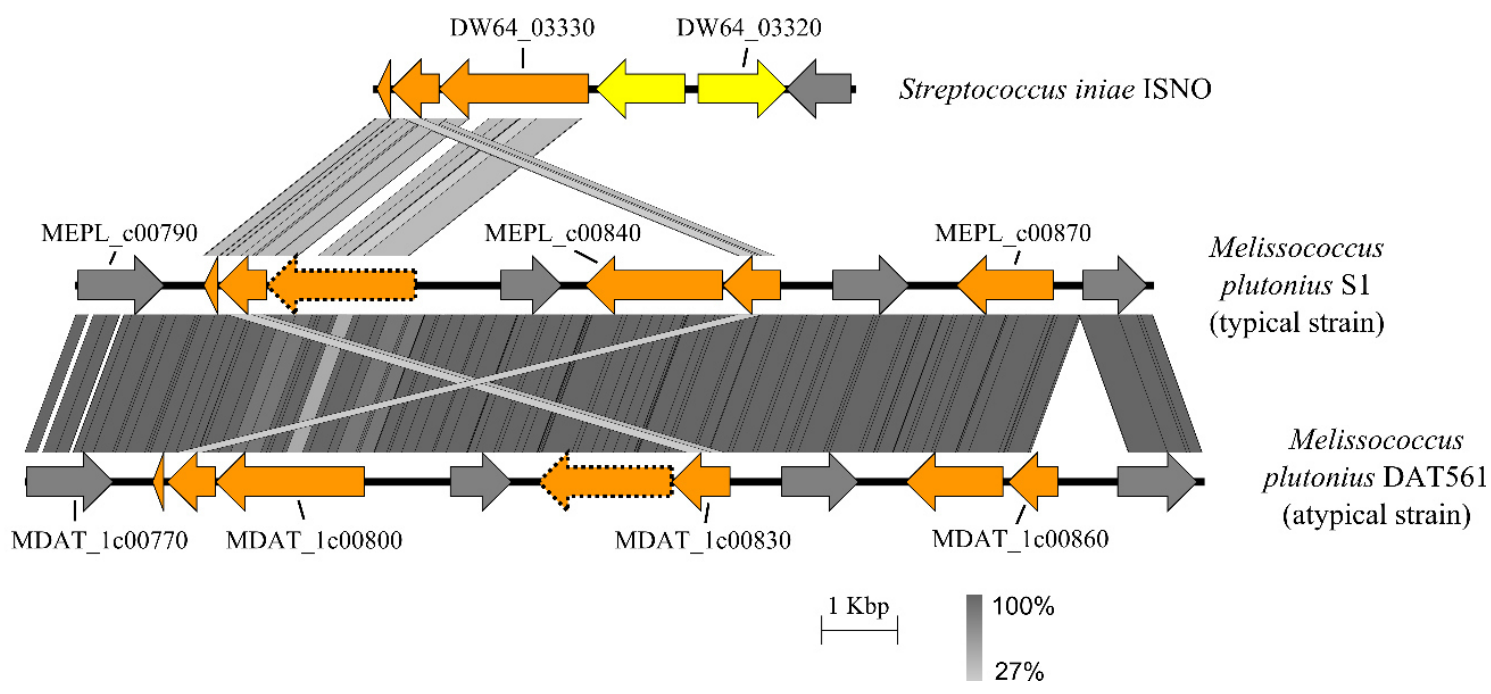

Supplementary Figure 1. Comparison of bacteriocin biosynthesis and transport clusters of $M$. plutonius S1with clusters of M. plutonius DAT561, Enterococcus faecalis FLY1 (accession nr. NZ_ACAR00000000) and Streptococcus iniae ISNO (accession nr. CP007587). The graphical presentation was done with the Easyfig software (minimum blast hit length of $50 \mathrm{bp}$ ) (Sullivan et al. 2011). ORFs related to bacteriocin biosynthesis and transport are orange-shaded, an iron uptake system is red-shaded, transposases are yellow and genomic context is shown in gray and black. ORFs depicted as dotted arrows represent pseudogenes. The bacteriocin biosynthesis cluster one (A) shows high similarity to a cluster present in E. faecalis FLY1, M. plutonius DAT561 lacks this region completely. Cluster two (B) represents a bacteriocin transport cluster. Three ORFs of cluster 2 share similarity to ORFs present in S. iniae ISNO. M. plutonius DAT561 encodes an additional ORF (MDAT_1c00860), encoding a transcriptional regulatory protein. M. plutonius S1 is chosen as a representative for all typical strains in this study for this analysis.

\section{Reference:}

Sullivan, M. J., Petty, N. K., and Beatson, S. A. (2011). Easyfig: a genome comparison visualizer. Bioinformatics 27, 1009-10. doi:10.1093/bioinformatics/btr039. 


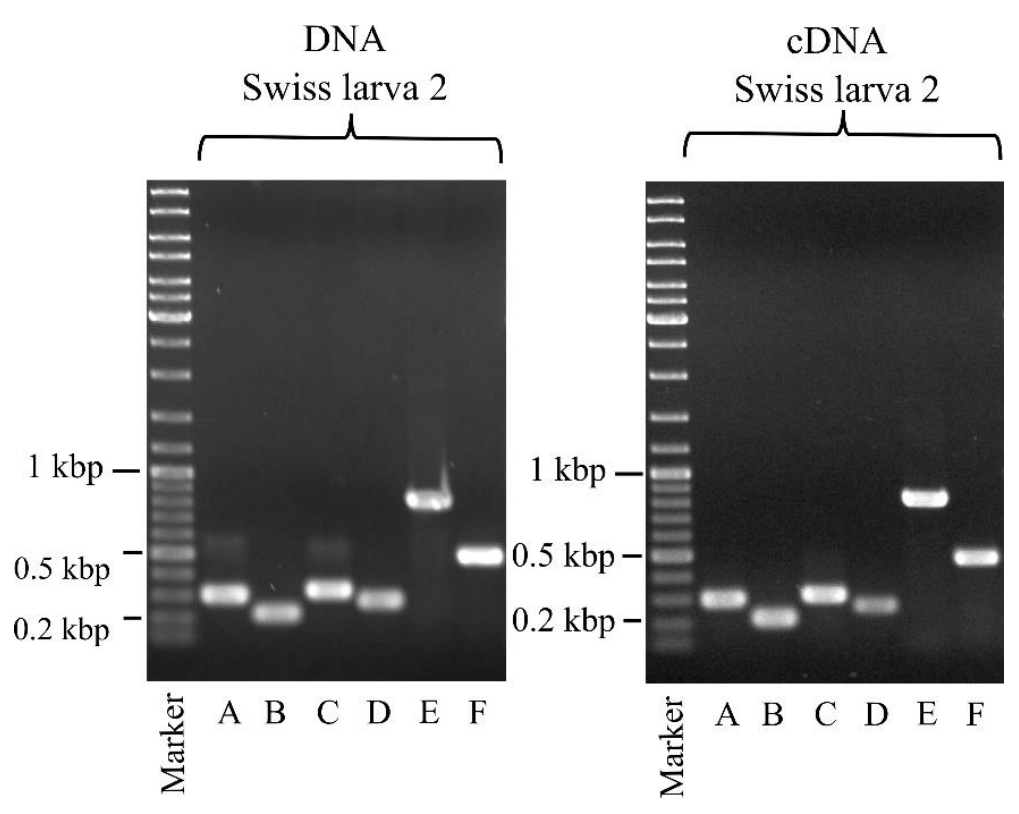

Supplementary Figure 2: Expression of $M$. plutonius putative virulence factors during infection. On the basis of isolated DNA and cDNA from EFB-infected and healthy larvae, we tested the expression of A - endo-alpha- $N$-acetylgalactosaminidase, B - enhancin, $\mathrm{C}$ - toxin, D - transcription termination factor $r h o, \mathrm{E}-16 \mathrm{~S}$ rRNA, F - RNA polymerase sigma factor rpoD and the 16S rRNA gene of M. plutonius via RT-PCR. In all EFB-infected larvae positive PCR products were obtained for DNA and cDNA. 


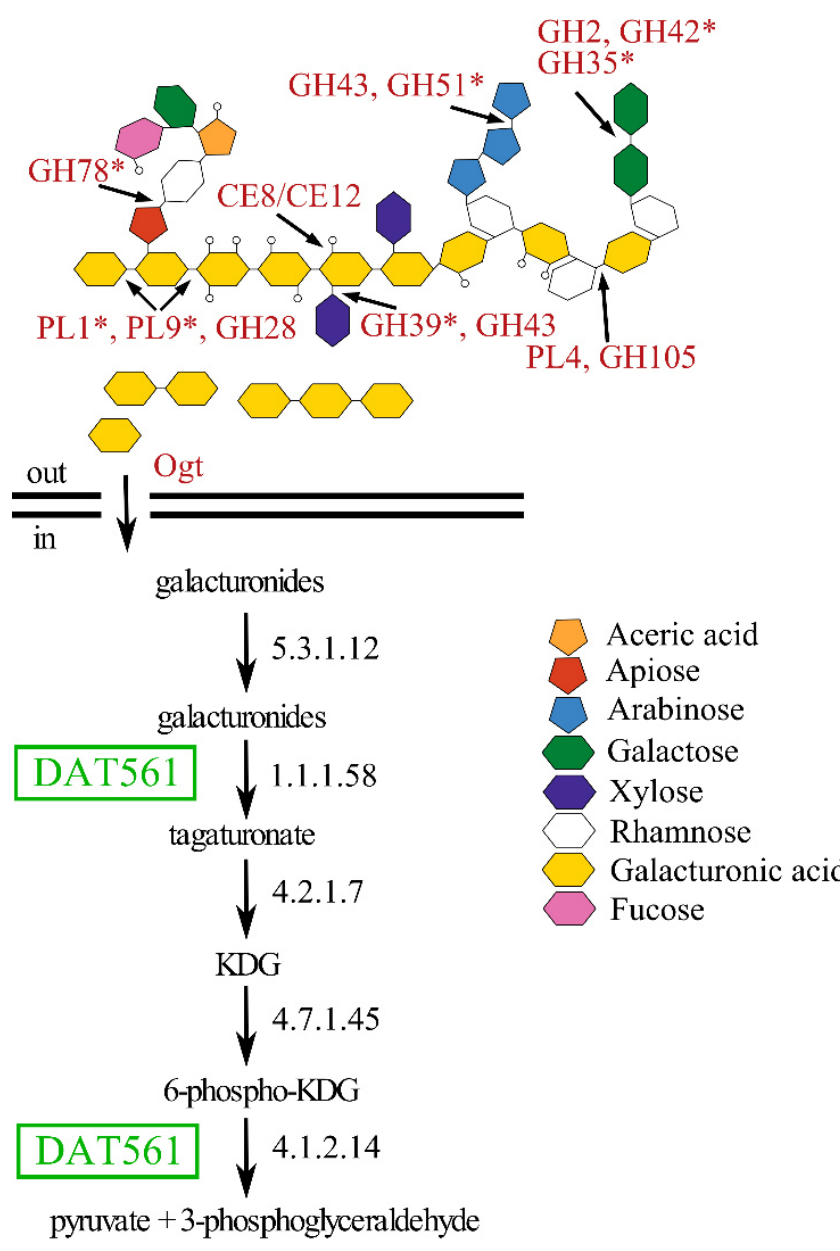

Reference:

Engel, P., Martinson, V. G., and Moran, N. A. (2012). Functional diversity within the simple gut microbiota of the honey bee. Proc. Natl. Acad. Sci. U. S. A. 109, 11002-7.
Supplementary Figure 3. Pectin degradation by $M$. plutonius. Adaptation of Figure 3A from Engel et al. (2012). A number of genes encoding plant cell walldegrading enzymes (including pollen walls) were identified in the genomes of all $M$. plutonius strains. Shown are identified families of glycoside hydrolases (GH), polysaccharide lyases (PL) and carbohydrate esterases (CE). Ogt is an acronym for oligogalacturonide transporter. Gene products are represented via EC numbers. Asterisks mark putative absence in at least one of the strains. The visualized pathway for galacturonide conversion could only be delineated with all components in M. plutonius DAT561, as genes encoding for tagaturonate reductase $\mathrm{UxaB}$ (EC 1.1.1.58) and 2-dehydro-3-deoxyphosphogluconate aldolase $\mathrm{KdgA}$ (EC 4.1.2.14) are putatively dysfunctional in the typical strains. PL1 is missing in $M$. plutonius DAT561. PL9, GH35, and GH42 are present only in strains 119, 90.0, 82, ATCC 35311, and DAT561. Additionally, a GH78 was detected only in DAT561, an extracellular GH51 is missing in strain 7656B, and a GH39 is present only in $M$. plutonius S1 and ATCC 35311. 


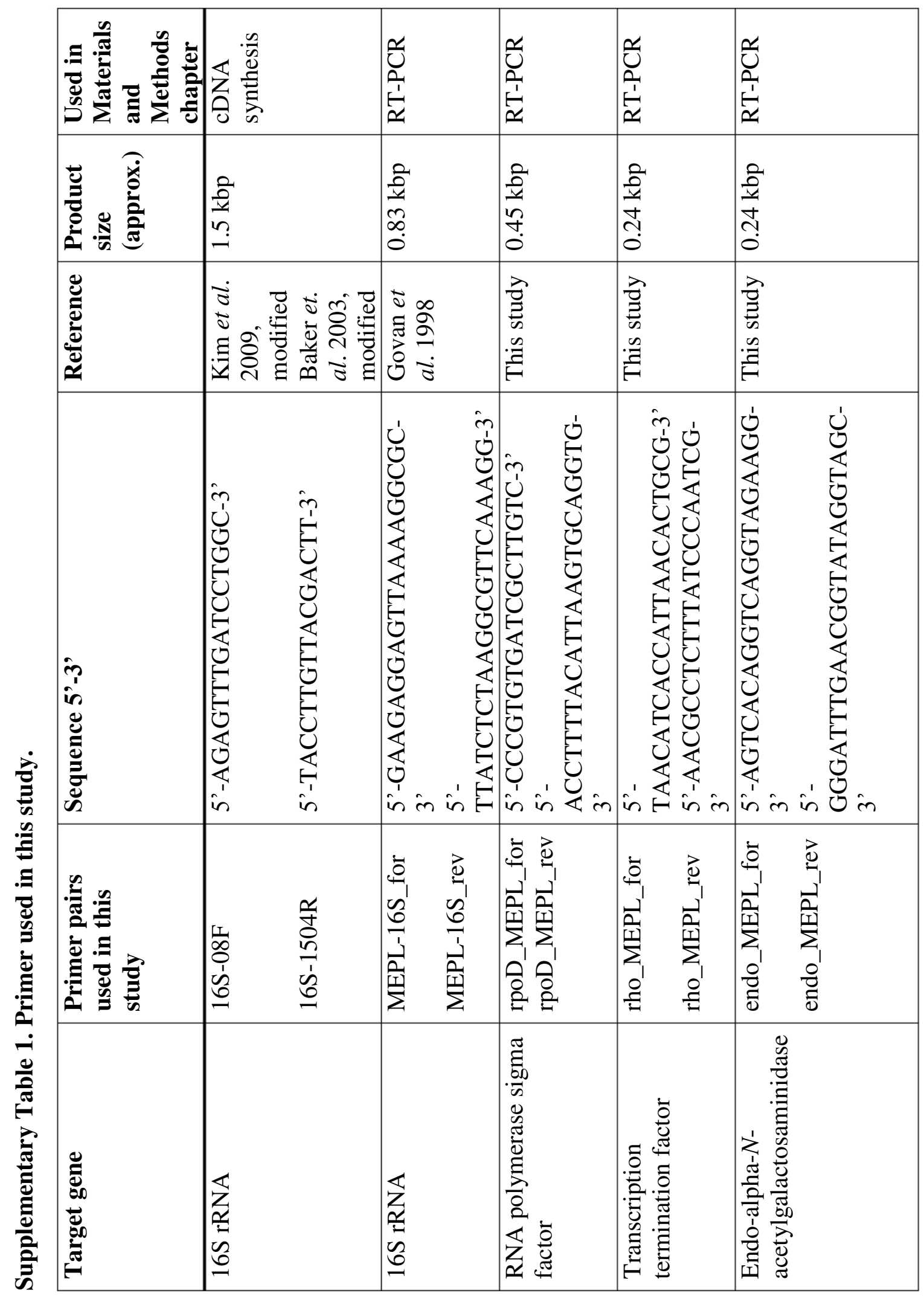




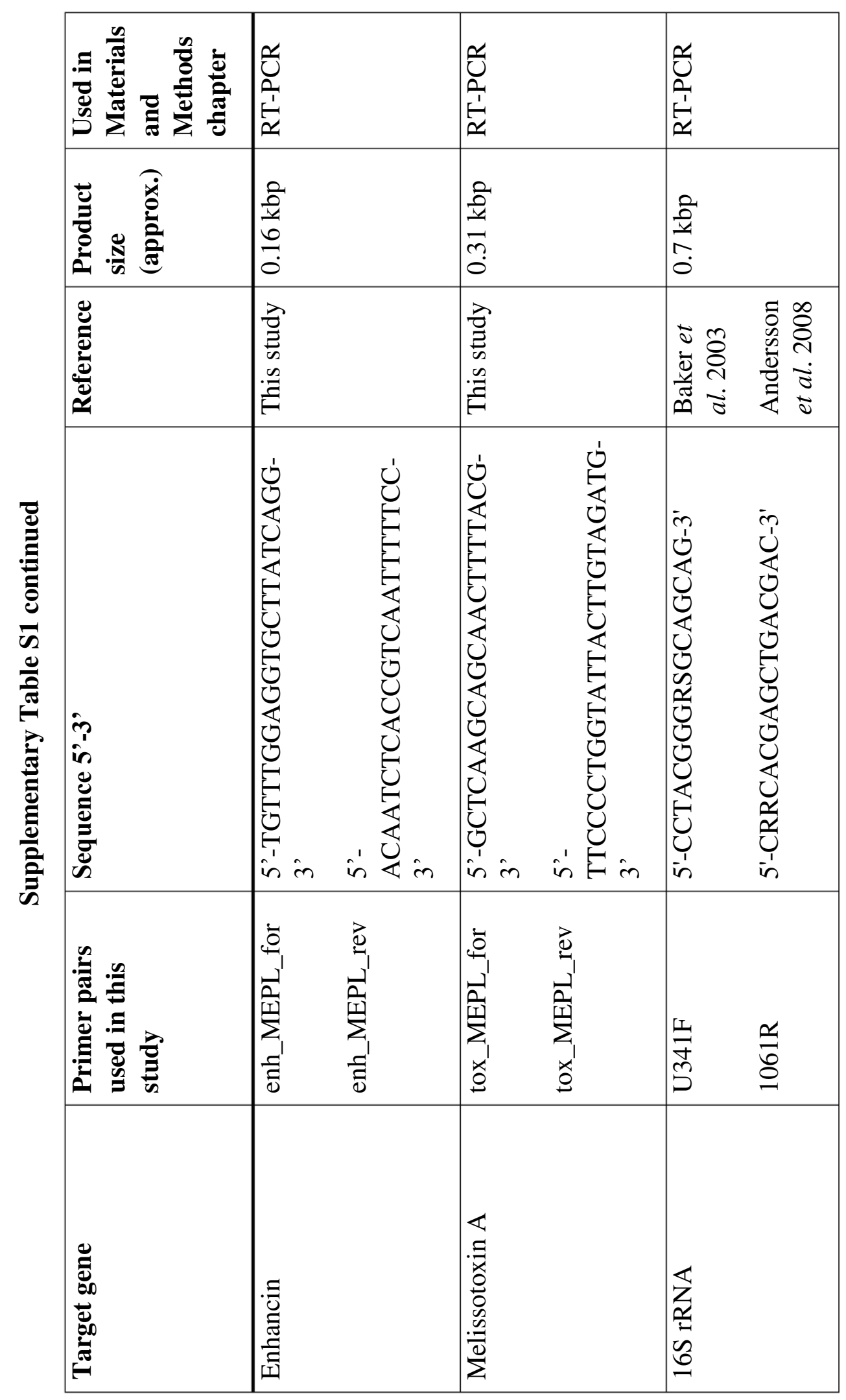




\section{References:}

Andersson, A. F., Lindberg, M., Jakobsson, H., Bäckhed, F., Nyrén, P., et al. (2008). Comparative analysis of human gut microbiota by barcoded pyrosequencing. PLoS One 3. doi:10.1371/journal.pone.0002836.

Baker, G. C., Smith, J. J., Cowan, D. A. (2003). Review and re-analysis of domain-specific 16S primers. J. Microbiol. Methods 55, 541-555. doi:10.1016/j.mimet.2003.08.009.

Govan, V. A., Brözel, V., Allsopp, M. H., Davison, S. (1998). A PCR detection method for rapid identification of Melissococcus pluton in honeybee larvae. Appl. Environ. Microbiol. 64, 1983-5.

Kim, Y. M., Ahn, C. K., Woo, S. H., Jung, G. Y., Park, J. M. (2009). Synergic degradation of phenanthrene by consortia of newly isolated bacterial strains. J. Biotechnol. 144, 293-298. doi:10.1016/j.jbiotec.2009.09.021. 


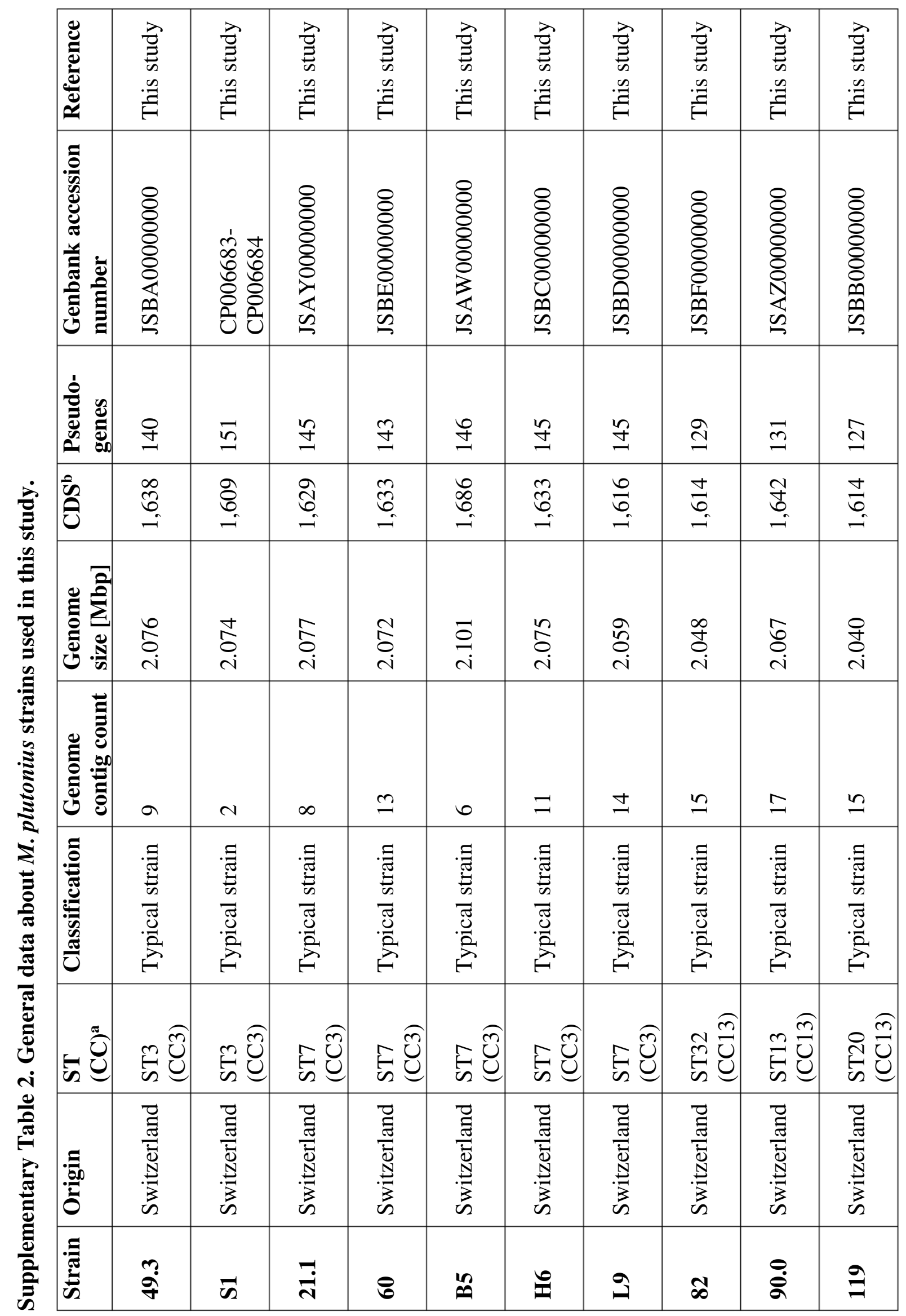




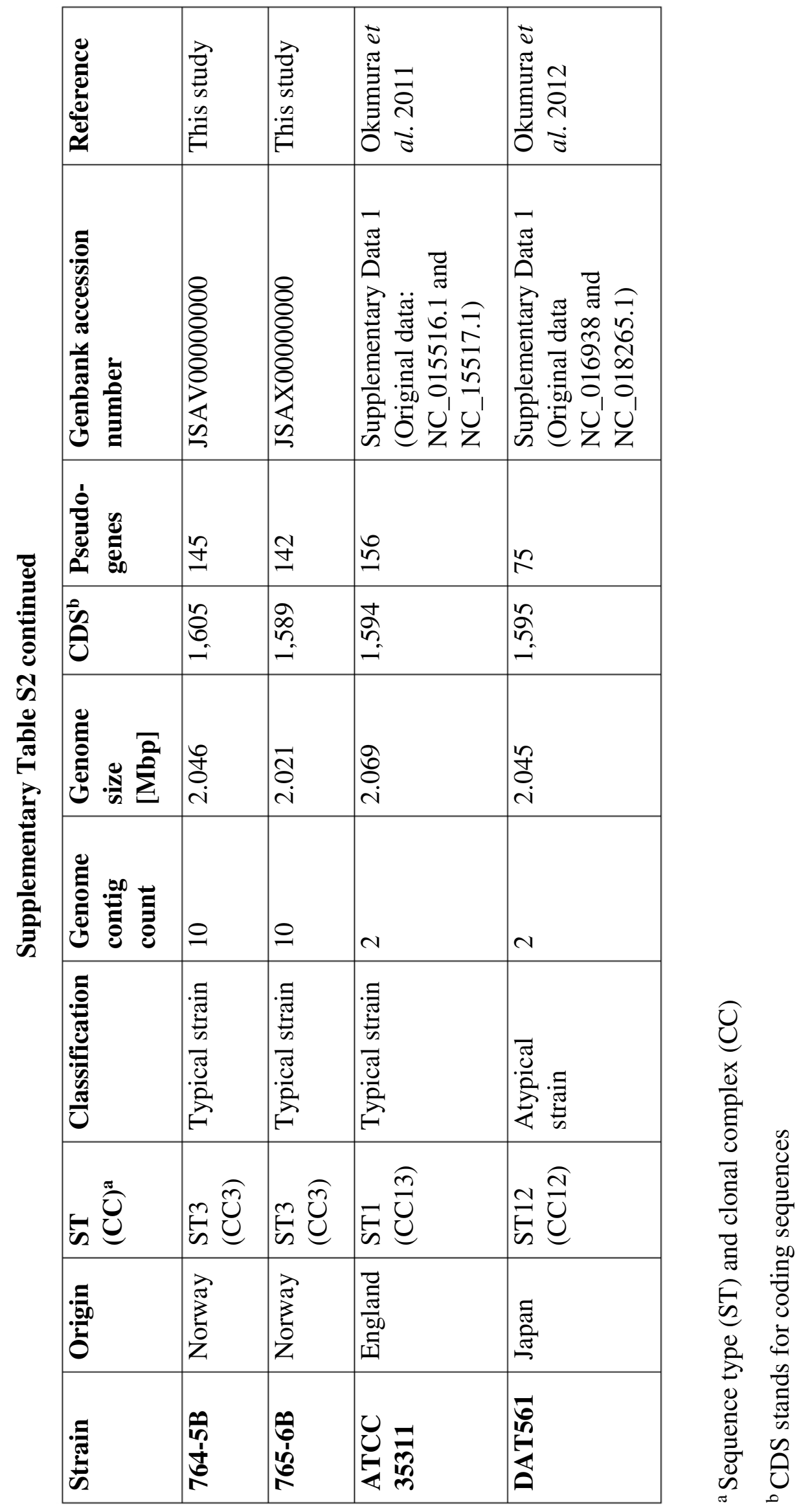




\section{References:}

Okumura, K., Arai, R., Okura, M., Kirikae, T., Takamatsu, D., et al. (2011). Complete genome sequence of Melissococcus plutonius ATCC 35311. J. Bacteriol. 193, 402930. doi:10.1128/JB.05151-11.

Okumura, K., Arai, R., Okura, M., Kirikae, T., Takamatsu, D., et al. (2012). Complete genome sequence of Melissococcus plutonius DAT561, a strain that shows an unusual growth profile and is representative of an endemic cluster in Japan. J. Bacteriol. 194, 3014. doi:10.1128/JB.00437-12. 


\title{
Genome Sequence of Brevibacillus laterosporus LMG 15441, a Pathogen of Invertebrates
}

\author{
Marvin Djukic ${ }^{1}$, Anja Poehlein ${ }^{1},{\text { Andrea } \text { Thürmer }^{1} \text { und Rolf Daniel }}^{1}$ \\ Journal of Bacteriology (2011), Vol. 193 (19), 5535-5536 \\ ${ }^{1}$ Abteilung für Genomische und Angewandte Mikrobiologie \& Göttingen Genomics \\ Laboratory, Institut für Mikrobiologie und Genetik, Georg-August Universität Göttingen, \\ Göttingen, Deutschland
}

\section{Anteilserklärung}

Idee/Konzept: AP, RD

Genomsequenzierung: AT

Lückenschluss und Datenauswertung: MD

Schreiben des Manuskripts: MD, AP, RD 


\title{
Genome Sequence of Brevibacillus laterosporus LMG 15441, a Pathogen of Invertebrates
}

\author{
Marvin Djukic, ${ }^{1}$ Anja Poehlein, ${ }^{1}$ Andrea Thürmer, ${ }^{1}$ and Rolf Daniel ${ }^{1,2 *}$ \\ Göttingen Genomics Laboratory ${ }^{1}$ and Department of Genomic and Applied Microbiology, ${ }^{2}$ Institute of Microbiology and \\ Genetics, Georg-August University of Göttingen, Grisebachstraße 8, D-37077 Göttingen, Germany
}

Received 29 June 2011/Accepted 18 July 2011

\begin{abstract}
Here we announce the genome sequence of the bacterium Brevibacillus laterosporus LMG 15441, which is a pathogen of invertebrates. The genome consists of one chromosome and two circular plasmids. Sequence analysis revealed a large potential to produce polyketides, nonribosomal peptides, and toxins.
\end{abstract}

Brevibacillus laterosporus (Br. laterosporus) (14), formerly classified as Bacillus laterosporus (12), is a Gram-positive and spore-forming aerobic bacterium, which is a pathogen of invertebrates (4). It has been found as a secondary invader during European foulbrood, which is a serious infectious disease of honey bees (6). The formation of canoe-shaped lamellar parasporal inclusions adjacent to spores is characteristic of $\mathrm{Br}$. laterosporus (10).

The genome sequence of Br. laterosporus LMG 15441 was determined by using a 454 GS-FLX system (Roche 454 Life Science, Mannheim, Germany). The initial assembly of three pyrosequencing shotgun runs yielded 84 contigs and 22-fold coverage. Closure of remaining gaps was performed by PCR and Sanger sequencing of the products. The genome of $\mathrm{Br}$. laterosporus comprises one chromosome $(5,106,578 \mathrm{bp})$, which is represented by a single scaffold (8 contigs), and two circular plasmids of 32,617 bp (pBRLA33) and 7,095 bp (pBRLA07). The chromosome, pBRLA33, and pBRLA07 encode 4,826, 36, and 9 predicted protein-encoding genes, respectively.

The genome sequence revealed that $\mathrm{Br}$. laterosporus has the potential to synthesize a variety of polyketides and nonribosomal peptides. It has been shown that Br. laterosporus is able to produce basiliskamides (1), tupuseleiamides (1), loloatins $(8,9)$, and tauramamide (5). Six putative hybrid clusters encoding polyketide synthases (PKS) and nonribosomal peptide synthetases (NRPS), five genes clusters for NRPS, and four single NRPS-encoding genes were identified. These genes and gene cluster are all located on the chromosome and comprise approximately $400 \mathrm{kbp}$. One NRPS/PKS-hybrid cluster (BRLA_c15910 to BRLA_c15820) shows 99\% DNA sequence identity to a nonribosomal peptide synthetase operon of Brevibacillus texasporus (GenBank accession no. AY953371), which encodes a peptide antibiotic. In addition to polyketides and nonribosomal peptides, the genome harbors genes encoding four putative toxins. One of these genes (BRLA_c21160) shows similarity to the $35.8-\mathrm{kDa}$ mosquitocidal toxin of Lysinibacillus sphaericus (GenBank accession no. AAB36655). The second gene (BRLA_c20010) revealed homology to the

* Corresponding author. Mailing address: Department of Genomic and Applied Microbiology and Göttingen Genomics Laboratory, Institute of Microbiology and Genetics, Georg-August University Göttingen, Grisebachstraße 8, D-37077 Göttingen, Germany. Phone: 49551-393827. Fax: 49-551-3912181. E-mail: rdaniel@gwdg.de. thiol-activated cytolysin alveolysin, which is able to destroy cholesterol-containing membranes (7). The other putative genes are related to binary toxins and each consist of two putative genes, which exhibit similarity to the protective antigen and lethal factor of Bacillus anthracis (2). Other potential virulence factors of $\mathrm{Br}$. laterosporus LMG 15441 include putative genes encoding collagenase, chitinases, virulence factor MviN, immune inhibitor A, and bacillolysin (3). In addition, the genome of Br. laterosporus harbors eight different genes coding for putative flagellins. Flagellin is the structural protein that forms the filament of bacterial flagella (11), but it is also a known virulence factor of pathogenic bacteria (13).

Nucleotide sequence accession number. The genome sequence of Br. laterosporus LMG 15441 has been deposited in GenBank under accession number AFRV00000000.

This work was supported by the German Federal Ministry of Education and Research (Bundesministerium für Bildung und Forschung $[\mathrm{BMBF}])$.

\section{REFERENCES}

1. Barsby, T., M. T. Kelly, and R. J. Andersen. 2002. Tupuseleiamides and basiliskamides, new acyldipeptides and antifungal polyketides produced in culture by a Bacillus laterosporus isolate obtained from a tropical marine habitat. J. Nat. Prod. 65:1447-1451.

2. Barth, H., K. Aktories, M. R. Popoff, and B. G. Stiles. 2004. Binary bacterial toxins: biochemistry, biology, and applications of common Clostridium and Bacillus proteins. Microbiol. Mol. Biol. Rev. 68:373-402.

3. Chung, M. C., et al. 2006. Secreted neutral metalloproteases of Bacillus anthracis as candidate pathogenic factors. J. Biol. Chem. 281:3140831418 .

4. de Oliveira, E. J., L. Rabinovitch, R. G. Monnerat, L. K. J. Passos, and V. Zahner. 2004. Molecular characterization of Brevibacillus laterosporus and its potential use in biological control. Appl. Environ. Microbiol. 70:6657-6664

5. Desjardine, K., et al. 2007. Tauramamide, a lipopeptide antibiotic produced in culture by Brevibacillus laterosporus isolated from a marine habitat: structure elucidation and synthesis. J. Nat. Prod. 70:1850-1853.

6. Forsgren, E. 2010. European foulbrood in honey bees. J. Invertebr. Pathol. 103:S5-S9.

7. Geoffroy, C., J. Mengaud, J. E. Alouf, and P. Cossart. 1990. Alveolysin, the thiol-activated toxin of Bacillus alvei, is homologous to listeriolysin O, perfringolysin $\mathrm{O}$, pneumolysin, and streptolysin $\mathrm{O}$ and contains a single cysteine. J. Bacteriol. 172:7301-7305.

8. Gerard, J., P. Haden, M. T. Kelly, and R. J. Andersen. 1996. Loloatin B, a cyclic decapeptide antibiotic produced in culture by a tropical marine bacterium. Tetrahedron Lett. 37:7201-7204.

9. Gerard, J. M., P. Haden, M. T. Kelly, and R. J. Andersen. 1999. Loloatins A-D, cyclic decapeptide antibiotics produced in culture by a tropical marine bacterium. J. Nat. Prod. 62:80-85

10. Huang, X. W., et al. 2005. An extracellular protease from Brevibacillus 
laterosporus $\mathrm{G} 4$ without parasporal crystals can serve as a pathogenic factor in infection of nematodes. Res. Microbiol. 156:719-727.

11. Kuwajima, G., J. I. Asaka, T. Fujiwara, K. Node, and E. Kondo. 1986 Nucleotide sequence of the hag gene encoding flagellin of Escherichia coli. J. Bacteriol. 168:1479-1483.

12. Laubach, C. A. 1916. Studies on aerobic spore-bearing non-pathogenic bacteria. Spore-bearing organisms in water. J. Bacteriol. 1:505-512.
13. Samakovlis, C., B. Asling, H. G. Boman, E. Gateff, and D. Hultmark 1992. In vitro induction of cecropin genes-an immune response in a Drosophila blood cell line. Biochem. Biophys. Res. Commun. 188:11691175 .

14. Shida, O., H. Takagi, K. Kadowaki, and K. Komagata. 1996. Proposal for two new genera, Brevibacillus gen nov and Aneurinibacillus gen nov. Int. J. Syst. Bacteriol. 46:939-946. 


\title{
Genome Sequence of Paenibacillus alvei DSM 29, a Secondary Invader during European Foulbrood Outbreaks
}

\author{
Marvin Djukic ${ }^{1}$, Dominik Becker ${ }^{1}$, Anja Poehlein ${ }^{1}$, Sonja Voget ${ }^{1}$ und Rolf Daniel $^{1}$
}

Journal of Bacteriology (2012), Vol. 194 (22), 6365

${ }^{1}$ Abteilung für Genomische und Angewandte Mikrobiologie \& Göttingen Genomics Laboratory, Institut für Mikrobiologie und Genetik, Georg-August Universität Göttingen, Göttingen, Deutschland

\section{Anteilserklärung}

Idee/Konzept: AP, RD

Genomsequenzierung: SV

Lückenschluss und Datenauswertung: MD, DB

Schreiben des Manuskripts: MD, AP, RD 


\section{Journal of Bacteriology}
Genome Sequence of Paenibacillus alvei DSM 29, a Secondary Invader during European Foulbrood Outbreaks

Marvin Djukic, Dominik Becker, Anja Poehlein, Sonja Voget and Rolf Daniel

J. Bacteriol. 2012, 194(22):6365. DOI: 10.1128/JB.01698-12.

Updated information and services can be found at:

http://jb.asm.org/content/194/22/6365

\begin{tabular}{cl}
\hline REFERENCES & $\begin{array}{l}\text { These include: } \\
\text { This article cites } 8 \text { articles, } 4 \text { of which can be accessed free at: } \\
\text { http://jb.asm.org/content/194/22/6365\#ref-list-1 }\end{array}$ \\
CONTENT ALERTS & $\begin{array}{l}\text { Receive: RSS Feeds, eTOCs, free email alerts (when new } \\
\text { articles cite this article), more» }\end{array}$ \\
\hline
\end{tabular}




\title{
Genome Sequence of Paenibacillus alvei DSM 29, a Secondary Invader during European Foulbrood Outbreaks
}

\author{
Marvin Djukic, Dominik Becker, Anja Poehlein, Sonja Voget, and Rolf Daniel \\ Department of Genomic and Applied Microbiology, and Göttingen Genomics Laboratory, Institute of Microbiology and Genetics, Georg-August University Göttingen, \\ Göttingen, Germany
}

Paenibacillus alvei is known as a secondary invader during European foulbrood of honeybees. Here, we announce the 6.83-Mb draft genome sequence of $P$. alvei type strain DSM 29. Putative genes encoding an antimicrobial peptide, a binary toxin, a mosquitocidal toxin, alveolysin, and different polyketides and nonribosomal peptides were identified.

Paeni form aenibacillus alvei is an aerobic, Gram-positive, and endosporeforming bacterium, which shows swarming activity on solidified culture media (5). P. alvei, Brevibacillus laterosporus, Enterococcus faecalis, and Achromobacter eurydice occur as secondary invaders of honeybees during outbreaks of European foulbrood (3). In addition, $P$. alvei is described as a causative agent of human infections (8).

The genome sequence of $P$. alvei DSM 29, which was isolated from foulbrood-diseased honeybees, was determined by using the 454 GS-FLX system and Titanium XL chemistry (Roche 454 Life Science, Mannheim, Germany). The initial assembly of one paired-end and three shotgun pyrosequencing runs yielded 266 contigs and 18-fold coverage. Closing of gaps was performed by PCR and Sanger sequencing of the resulting products. The draft genome $(6.83 \mathrm{Mb})$ consists of 25 contigs, comprising one chromosome and at least four plasmids (pPAV14, pPAV16, pPAV109, and pPAV141). The genome ( $45.9 \mathrm{~mol} \% \mathrm{G}+\mathrm{C}$ content) contains approximately 6,605 predicted protein-encoding genes.

Analysis of the $P$. alvei genome sequence revealed that this organism bears the potential to produce a variety of polyketides and nonribosomal peptides. At least six putative gene clusters encoding nonribosomal peptide synthetases, three single nonribosomal peptide synthetase-encoding genes, and five putative hybrid clusters harboring polyketide synthases and nonribosomal peptide synthetases were identified.

We found 10 different putative genes coding for chitin-degrading enzymes. Chitin stabilizes the cuticles of the epidermis and trachea as well as the peritrophic matrix in insects (7). Hyaluronate lyases are known virulence factors, as they are able to degrade the connective tissue of eukaryotes (6). We identified one putative hyaluronate lyase (PAV_3c01910), which, together with chitindegrading enzymes, might contribute to the invasive capacity of the pathogen.

The genome of $P$. alvei harbors putative genes for one antimicrobial peptide (PAV_2c01250 to PAV_2c01270) and different toxins such as one binary toxin (PAV_1c12540, PAV_1c12550), one putative mosquitocidal toxin (PAV_2c04820), and alveolysin (PAV_7c02420). The latter is a thiol-activated membranolytic toxin (4). In addition, other genes are related to a toxin-antitoxin system (PAV_109p01200), hemolysin III-like proteins, and an insecticidal toxin complex. The insecticidal toxin complex produces orally active toxins, which are located on the surface of the toxinproducing pathogen (2). Three components (A to C) are required to exhibit full toxicity. The complete operon coding for the insecticidal toxin complex of $P$. alvei shows high similarity to the tca operon of Bacillus thuringiensis IBL 200 (1). However, the gene region of $P$. alvei shows some differences in gene organization compared to that of $B$. thuringiensis. In $P$. alvei, the genes encoding component A are duplicated (PAV_1c12470 and PAV_1c12480, PAV_1c12500 and PAV1c_12510) and component $\mathrm{B}$ is encoded by two separate open reading frames (PAV_1c12450 and PAV_1c12460).

Nucleotide sequence accession number. The genome sequence of $P$. alvei DSM 29 has been deposited in GenBank under accession number AMBZ00000000.

\section{ACKNOWLEDGMENT}

This work was partly supported by the German Federal Ministry of Education and Research (Bundesministerium für Bildung und Forschung $[\mathrm{BMBF}])$.

\section{REFERENCES}

1. Blackburn MB, Martin PAW, Kuhar D, Farrar RR, Gundersen-Rindal DE. 2011. The occurrence of Photorhabdus-like toxin complexes in Bacillus thuringiensis. PLoS One 6:e18122. doi:10.1371/journal.pone.0018122.

2. ffrench-Constant RH, Dowling A, Waterfield NR. 2007. Insecticidal toxins from Photorhabdus bacteria and their potential use in agriculture. Toxicon 49:436-451.

3. Forsgren E. 2010. European foulbrood in honey bees. J. Invertebr. Pathol. 103:S5-S9.

4. Geoffroy C, Mengaud J, Alouf JE, Cossart P. 1990. Alveolysin, the thiolactivated toxin of Bacillus alvei, is homologous to listeriolysin O, perfringolysin $\mathrm{O}$, pneumolysin, and streptolysin $\mathrm{O}$ and contains a single cysteine. J. Bacteriol. 172:7301-7305.

5. Kim Y-G, Lee J-H, Cho MH, Lee J. 2011. Indole and 3-indolylacetonitrile inhibit spore maturation in Paenibacillus alvei. BMC Microbiol. 11:119.

6. Li S, Jedrzejas MJ. 2001. Hyaluronan binding and degradation by Streptococcus agalactiae hyaluronate lyase. J. Biol. Chem. 276:41407-41416.

7. Merzendorfer H. 2003. Chitin metabolism in insects: structure, function and regulation of chitin synthases and chitinases. J. Exp. Biol. 206:43934412.

8. Reboli AC, Bryan CS, Farrar WE. 1989. Bacteremia and infection of a hip prosthesis caused by Bacillus alvei. J. Clin. Microbiol. 27:1395-1396.

Received 10 September 2012 Accepted 13 September 2012 Address correspondence to Rolf Daniel, rdaniel@gwdg.de. Copyright (C) 2012, American Society for Microbiology. All Rights Reserved. doi:10.1128/JB.01698-12 


\title{
High quality draft genome of Lactobacillus kunkeei EFB6, isolated from a German European foulbrood outbreak of honeybees
}

\begin{abstract}
Marvin Djukic ${ }^{1}$, Anja Poehlein ${ }^{1}$, Juliane Strau $\beta^{1}$, Fabian Jannik Tann ${ }^{1}$, Andreas
\end{abstract} Leimbach $^{1}$, Michael Hoppert ${ }^{2}$ und Rolf Daniel ${ }^{1}$

Standards in Genomic Sciences (2015), Vol. 10, 16

\footnotetext{
${ }^{1}$ Abteilung für Genomische und Angewandte Mikrobiologie \& Göttingen Genomics Laboratory, Institut für Mikrobiologie und Genetik, Georg-August Universität Göttingen, Göttingen, Deutschland

${ }^{2}$ Abteilung für Allgemeine Mikrobiologie, Institut für Mikrobiologie und Genetik, GeorgAugust Universität Göttingen, Göttingen, Deutschland
}

\section{Anteilserklärung}

Idee/Konzept: MD, AP, RD

Planung der mikrobiologischen Experimente: MD, AP

Durchführung mikrobiologischer Experimente: MD, JS, FJT, MH

Genomsequenzierung: AP

Datenprozessierung: MD, AL, AP

In silico Genomanalyse: MD

Schreiben des Manuskripts: MD, AP, MH, RD 


\title{
High quality draft genome of Lactobacillus kunkeei EFB6, isolated from a German European foulbrood outbreak of honeybees
}

\author{
Marvin Djukic ${ }^{1}$, Anja Poehlein ${ }^{1}$, Juliane Strauß ${ }^{1}$, Fabian Jannik Tann ${ }^{1}$, Andreas Leimbach¹, Michael Hoppert ${ }^{2}$ \\ and Rolf Daniel ${ }^{1 *}$
}

\begin{abstract}
The lactic acid bacterium Lactobacillus kunkeei has been described as an inhabitant of fructose-rich niches. Here we report on the genome sequence of L. kunkeei EFB6, which has been isolated from a honeybee larva infected with European foulbrood. The draft genome comprises 1,566,851 bp and 1,417 predicted protein-encoding genes.
\end{abstract}

Keywords: Lactobacillus kunkeei, Lactic acid bacteria, European foulbrood, Honeybee, Cellular surface protein, Biofilm formation

\section{Introduction}

Honeybees are the most economically valuable pollinators of agricultural crops [1]. A disappearance of honeybees would result in an approximately $90 \%$ decrease in production of some fruits [2]. European foulbrood (EFB) and American foulbrood (AFB) are the two most important honeybee diseases affecting the brood [3]. While the AFB is caused by the spore-forming, Gram positive bacterium Paenibacillus larvae [4], EFB is caused by the capsuleproducing Melissococcus plutonius [5]. It has been shown that members of the lactic acid bacteria (LABs) inhibit the growth of M. plutonius [6] and P. larvae [7]. LABs are found in a variety of habitats, including human and animal microbiomes, and are used as food additives.

The honeybee crop microbiome consists of 13 bacterial species belonging to the genera Lactobacillus and Bifidobacterium [8]. These bacteria play a key role in the production of honey and bee bread. The latter serves as long-term food storage for adult honeybees and larvae. L. kunkeei is a common symbiont for Apis and the dominating $\mathrm{LAB}$ member in bees [6]. The organism is a specialist for colonization of the honeybee crop and

\footnotetext{
* Correspondence: rdaniel@gwdg.de

'Department of Genomic and Applied Microbiology \& Göttingen Genomics Laboratory, Institute of Microbiology and Genetics, Georg-August University of Göttingen, Göttingen, Germany

Full list of author information is available at the end of the article
}

interacts with the epithelial layer of the crop. L. kunkeei has been described as a fructophilic LAB [9]. Initially, it was isolated from wine [10], but it has also been found on flowers and in honey.

L. kunkeei EFB6 is the first LAB isolated from a German EFB-diseased larva. Here, we describe genomic features of this organism, focusing on factors that improve competition with bacteria such as M. plutonius and P. larvae. In addition, potential cell surface proteins that might play a role in cellular adhesion and biofilm formation are analyzed.

\section{Organism information}

In October 2012, an EFB outbreak in Bavaria (Germany) was confirmed. EFB-diseased larvae from this outbreak were collected, immediately frozen in liquid nitrogen and stored at $-80^{\circ} \mathrm{C}$ for further investigation. Several EFBinfected larvae were dissected under sterile conditions. To obtain LAB the guts of the larvae, which formed a yellow, glue-like slime, were suspended in MRS medium (Carl Roth GmbH \& Co KG, Karlsruhe, Germany) and subsequently streaked on solidified MRS to isolate single colonies. Strain L. kunkeei EFB6 (Table 1, Additional file 1: Table S1) was isolated from these agar plates after aerobic incubation at $35^{\circ} \mathrm{C}$.

L. kunkeei EFB6 is a non-sporulating, low G + C Gram positive member of the Lactobacteriaceae and taxonomically related to the genus Pediococcus. The strain exhibited 


\begin{tabular}{|c|c|c|c|}
\hline MIGS ID & Property & Term & Evidence code \\
\hline & Classification & Domain Bacteria & TAS [11] \\
\hline & & Phylum Firmicutes & TAS [12-15] \\
\hline & & Class Bacilli & TAS [16] \\
\hline & & Order Lactobacillales & TAS [17] \\
\hline & & Family Lactobacillaceae & TAS [18] \\
\hline & & Genus Lactobacillus & TAS [18-21] \\
\hline & & Species Lactobacillus kunkeei & TAS [10] \\
\hline & & strain: EFB6 & TAS (this study) \\
\hline & Gram stain & Positive & TAS [10] \\
\hline & Cell shape & Rod-shaped & IDA \\
\hline & Motility & Non-motile & IDA \\
\hline & Sporulation & Non-sporulating & NAS \\
\hline & Temperature range & Mesophile & TAS [10] \\
\hline & Optimum temperature & $30^{\circ} \mathrm{C}$ & NAS \\
\hline & $\mathrm{pH}$ range; Optimum & $4.5-6.2 ; 6$ & NAS \\
\hline & Carbon source & Varied & NAS \\
\hline MIGS-6 & Habitat & Honeybee larva & IDA \\
\hline MIGS-6.3 & Salinity & $5 \% \mathrm{NaCl}(\mathrm{w} / \mathrm{v})$ & TAS [10] \\
\hline MIGS-22 & Oxygen requirement & Facultative & IDA \\
\hline MIGS-15 & Biotic relationship & Host-associated & TAS [6] \\
\hline \multirow[t]{2}{*}{ MIGS-14 } & Pathogenicity & Non-pathogen & NAS \\
\hline & Biosafety level & 1 & TAS [22] \\
\hline MIGS-23 & Isolation & EFB-diseased honeybee larva & IDA \\
\hline MIGS-4 & Geographic location & Bavaria, Germany & IDA \\
\hline MIGS-5 & Sample collection & October 1, 2012 & IDA \\
\hline MIGS-4.1 & Latitude & $49^{\circ} 14^{\prime} \mathrm{N}$ & IDA \\
\hline MIGS-4.2 & Longitude & $11^{\circ} 05^{\prime} \mathrm{E}$ & IDA \\
\hline MIGS-4.4 & Altitude & $400 \mathrm{~m}$ a.s.l & IDA \\
\hline
\end{tabular}

a $100 \% 16 \mathrm{~S}$ rRNA gene nucleotide sequence identity to the type strain L. kunkeei YH-15 (Table 1, Figure 1). Cells harvested in exponential growth phase exhibited a length ranging from 0.7 to $1.3 \mu \mathrm{m}$ and a diameter ranging from 0.3 to $0.5 \mu \mathrm{m}$ as determined by transmission electron microscopy (TEM) of either negatively stained or ultrathin-sectioned samples (Figure 2). Preparations for ultrathin sectioning and negative staining of cells were performed as described by [23]. The L. kunkeei EFB6 cell wall is approximately $12 \mathrm{~nm}$ thick. This value is rather thin compared to cell walls of other Gram positives [24]. Three distinct wall layers of L. kunkeei EFB6 (two darker stained outer and inner layers and a brighter layer in between) could be distinguished by TEM. Surface layers and cellular appendages (pili, fimbriae) were not detected.

\section{Genome sequencing and annotation}

\section{Genome project history}

The organism was selected for sequencing on the basis of its use as potential inhibitor for the primary agents of AFB and EFB [6,7]. The aim was to investigate potential factors to increase bacterial competition fitness and cell surface proteins, which might be important for cellular adhesion and biofilm formation.

A summary of the project information is shown in Table 2.

\section{Growth conditions and DNA isolation}

To isolate genomic DNA L. kunkeei EFB6 was grown aerobically in $50 \mathrm{ml}$ MRS medium at $35^{\circ} \mathrm{C}$ with shaking at $150 \mathrm{rpm}$ (Lab-Therm Lab-Shaker, Adolf Kühner AG, Birsfelden, Switzerland). Cells were harvested in 


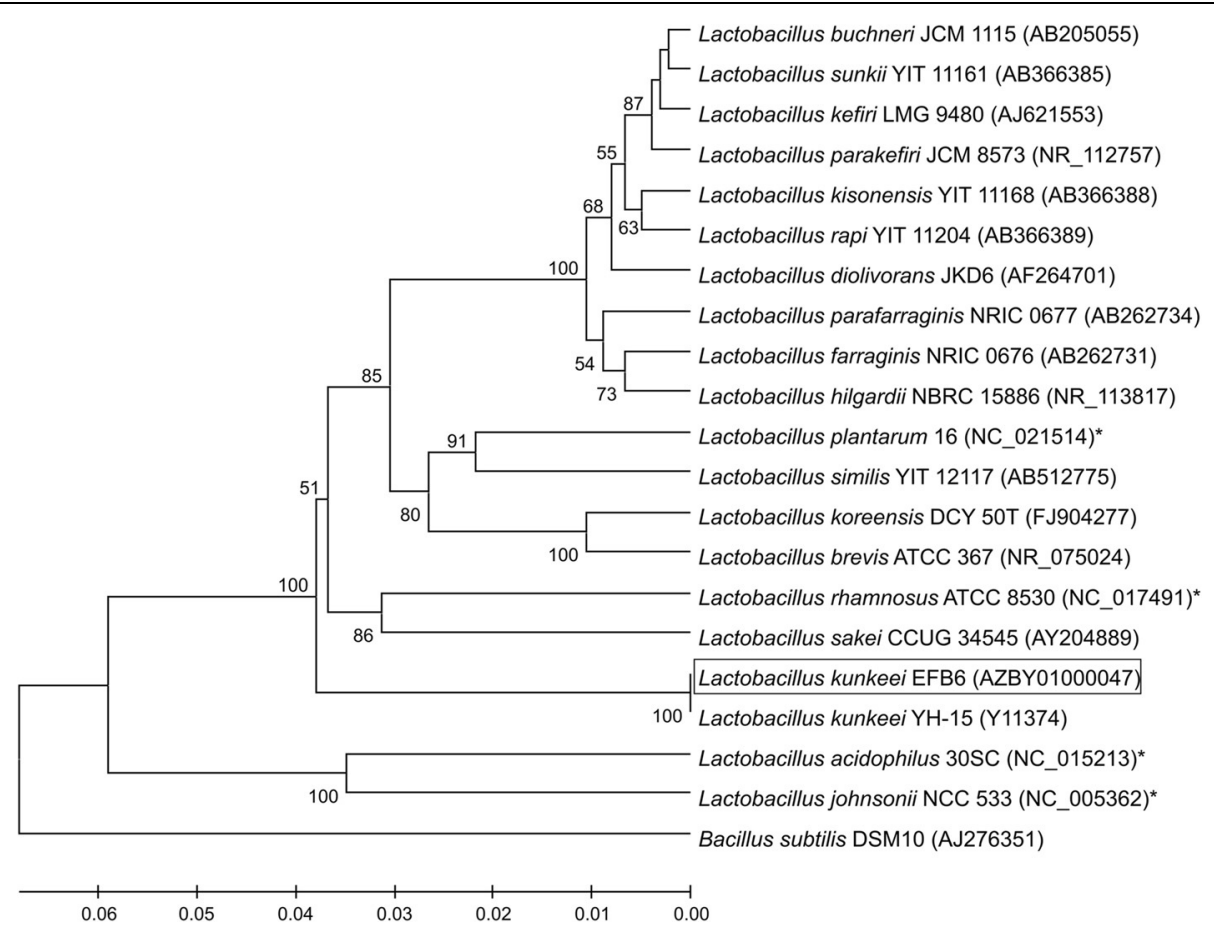

Figure 1 Phylogenetic tree highlighting the position of L. kunkeei EFB6 relative to other Lactobacillus strains based on 16S rRNA gene sequences. GenBank accession numbers are indicated in parentheses. Asterisks indicate that a consensus sequence was calculated from all $16 \mathrm{~S}$ rRNA gene sequences present in the corresponding genome. L. kunkeei EFB6 is boxed. Sequences were aligned using ClustalW 1.6 [25]. The phylogenetic tree was obtained by using the UPGMA method within MEGA 6.06 software [26]. Numbers at nodes are bootstrap values calculated from 1,000 resamplings to generate a majority consensus tree. Bacillus subtilis DSM10 was used as outgroup. The scale bar indicates the nucleotide sequence divergence.

exponential growth phase using a Beckman Coulter Allegra $^{\text {Tx }} \mathrm{X}-12 \mathrm{R}$ centrifuge (Beckman Coulter $\mathrm{GmbH}$, Krefeld, Germany) for 25 minutes at 2,750 $g$ and $4^{\circ} \mathrm{C}$. Genomic DNA was isolated using the Epicentre ${ }^{\circ}$ MasterPure $^{\mathrm{m}}$ DNA Purification kit (Epicentre ${ }^{\circ}$, Madison, WI, USA).

\section{Genome sequencing and assembly}

Whole-genome sequencing of L. kunkeei EFB6 was performed by employing the Genome Analyzer II (Illumina, San Diego, CA). The shotgun library was prepared according to the manufacturer's protocols. For de novo

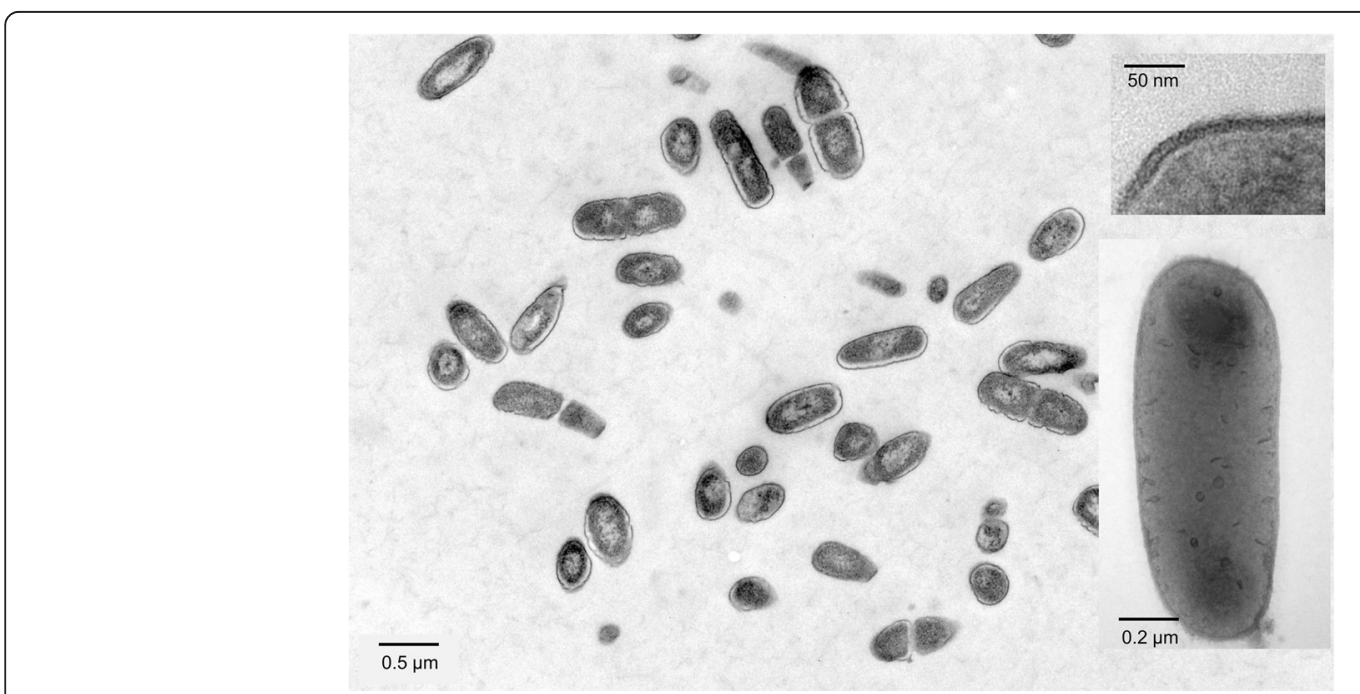

Figure 2 Electron microscopy of $L$. kunkeei EFB6. Large image and upper right inset: stained ultrathin sections; lower right inset: negatively stained single cell (staining salt: uranyl acetate, $4 \%, \mathrm{w} / \mathrm{v})$. 
Table 2 Genome sequencing project information

\begin{tabular}{|c|c|c|}
\hline MIGS ID & Property & Term \\
\hline MIGS-31 & Finishing quality & Improved high-quality draft \\
\hline MIGS-28 & Libraries used & $\begin{array}{l}\text { One Illumina paired-end } \\
\text { library with1 kb insert size }\end{array}$ \\
\hline MIGS-29 & Sequencing platforms & Illumina GAll \\
\hline MIGS-31.2 & Fold coverage & $142.96 \times$ Illumina \\
\hline MIGS-30 & Assemblers & SPAdes 2.5 \\
\hline \multirow[t]{8}{*}{ MIGS-32 } & Gene calling method & YACOP, Glimmer \\
\hline & Locus Tag & LAKU \\
\hline & Genbank ID & AZBY00000000 \\
\hline & GenBank Date of Release & May, 2014 \\
\hline & GOLD ID & Gi0053745 \\
\hline & NCBI project ID & 227106 \\
\hline & BIOPROJECT & PRJNA227106 \\
\hline & Project relevance & Host-associated \\
\hline
\end{tabular}

assembly, we used 2,000,000 paired-end Illumina reads (112 bp) and the SPAdes 2.5 software [27]. The final assembly contained 55 contigs larger than $500 \mathrm{bp}$ and revealed an average coverage of 142.96 .

\section{Genome annotation}

For automatic gene prediction the software tools YACOP [28] and Glimmer [29] were used. Identification of rRNA and tRNA genes was performed by employing RNAmmer [30] and tRNAscan [31], respectively. The annotation provided by the IMG-ER system [32] was corrected manually. For this purpose, data obtained from different databases (Swiss-Prot [33], TrEMBL [34] and InterPro [35]) were used to improve the quality of the annotation.

\section{Genome properties}

The genome statistics are provided in Table 3. The high quality draft genome sequence consists of 55 contigs that account for a total of $1,566,851 \mathrm{bp}$ and a $\mathrm{G}+\mathrm{C}$ content of $37 \mathrm{~mol} \%$. Of the 1,455 predicted genes, 1,417 were putatively protein-encoding, 35 represented putative tRNA genes and three putative rRNA genes. For the majority of the protein-encoding genes $(75 \%)$ a function could be assigned. The distribution of these genes into COG functional categories [36] is shown in Table 4.

\section{Insights into the genome}

Five different Lactobacillus species were used for genome comparisons with L. kunkeei EFB6 based on blastp [37]. Results are shown in Figure 3. All five species are of interest as probiotics, part of the gastrointestinal tract of animals or humans, or used in the production of fermented food.

The identification of orthologous proteins was performed with the program Proteinortho 5.04 [39] by using
Table 3 Genome statistics

\begin{tabular}{lr}
\hline Attribute & \multicolumn{1}{c}{ Value } \\
\hline Genome size (bp) & $1,566,851$ \\
DNA coding (bp) & $1,413,077$ \\
DNA G + C (bp) & 578,359 \\
DNA scaffolds & 55 \\
Total genes & 1,455 \\
Protein coding genes & 1,417 \\
RNA genes & 38 \\
Pseudo Genes & 0 \\
Genes in internal clusters & 20 \\
Genes with function prediction & 1,012 \\
Genes assigned to COGs & 1,195 \\
Genes assigned Pfam domains & 1,221 \\
Genes with signal peptides & 62 \\
Genes with transmembrane helices & 419 \\
CRISPR repeats & 0 \\
\hline
\end{tabular}

Table 4 Number of genes associated with the general COG functional categories

\begin{tabular}{lrrll}
\hline Code & Value & \% age & Description \\
\hline A & 137 & 10.57 & Translation, ribosomal structure and biogenesis \\
K & 0 & 0.00 & RNA processing and modification \\
L & 95 & 7.33 & Transcription \\
B & 94 & 7.25 & Replication, recombination and repair \\
D & 0 & 0.00 & Chromatin structure and dynamics \\
& 24 & 1.85 & Cell cycle control, cell division, chromosome \\
V & 18 & 1.39 & Defense mechanisms \\
T & 32 & 2.47 & Signal transduction mechanisms \\
M & 88 & 6.79 & Cell wall/membrane biogenesis \\
N & 10 & 0.77 & Cell motility \\
U & 25 & 1.93 & Intracellular trafficking and secretion \\
O & 45 & 3.47 & Posttranslational modification, protein \\
& & & turnover, chaperones \\
C & 49 & 3.78 & Energy production and conversion \\
G & 67 & 5.17 & Carbohydrate transport and metabolism \\
E & 112 & 8.64 & Amino acid transport and metabolism \\
F & 68 & 5.25 & Nucleotide transport and metabolism \\
H & 34 & 2.62 & Coenzyme transport and metabolism \\
I & 35 & 2.70 & Lipid transport and metabolism \\
P & 61 & 4.71 & Inorganic ion transport and metabolism \\
Q & 13 & 1.00 & Secondary metabolites biosynthesis, \\
& & & transport and catabolism \\
R & 155 & 11.96 & General function prediction only \\
S & 134 & 10.34 & Function unknown \\
- & 260 & 17.87 & Not in CoGs \\
\hline & & & \\
\hline
\end{tabular}




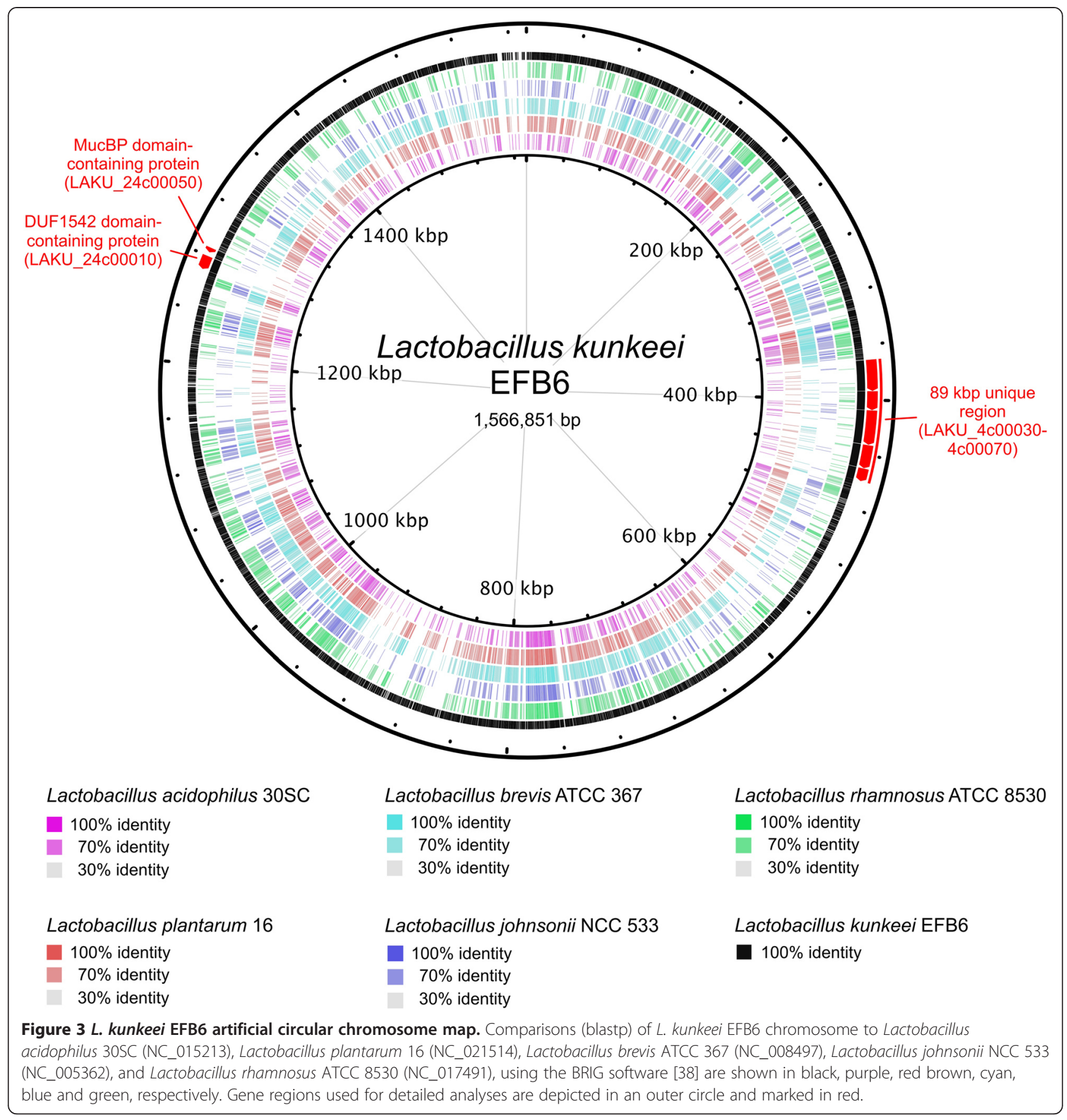

the protein content deduced from 232 lactobacilli genomes as references (GenBank database as of 28.02.2014). For this purpose ncbi_ftp_download v0.2, cat_seq v0.1 and cds_extractor v0.6 were used [40]. With an identity cutoff of 50\%, we identified 425 proteins in L. kunkeei EFB6 without orthologs in any other Lactobacillus species. Among these unique L. kunkeei EFB6 proteins, we selected 7 proteins for detailed analyses.

Analysis of the $89-\mathrm{kb}$ region shown in Figure 3 revealed five ORFs (LAKU_4c00030-LAKU_4c00070) without orthologs in any genomes derived from lactobacilli deposited in GenBank (as of 28.02.2014). Furthermore, no homologs could be identified in any other sequenced microbial genome (NCBI nr-database as of 05.03.2014) by using blastp (e-value cutoff of 1e-20). Except for LAKU_4c00060 (7,521 amino acids), we could identify an N-terminal signal peptide and a noncytoplasmic domain (Figure 4A) using Phobius' domain prediction software [41]: LAKU_4c00040 (4,579 amino acids) and LAKU_4c00070 (3,129 amino acids) contain 


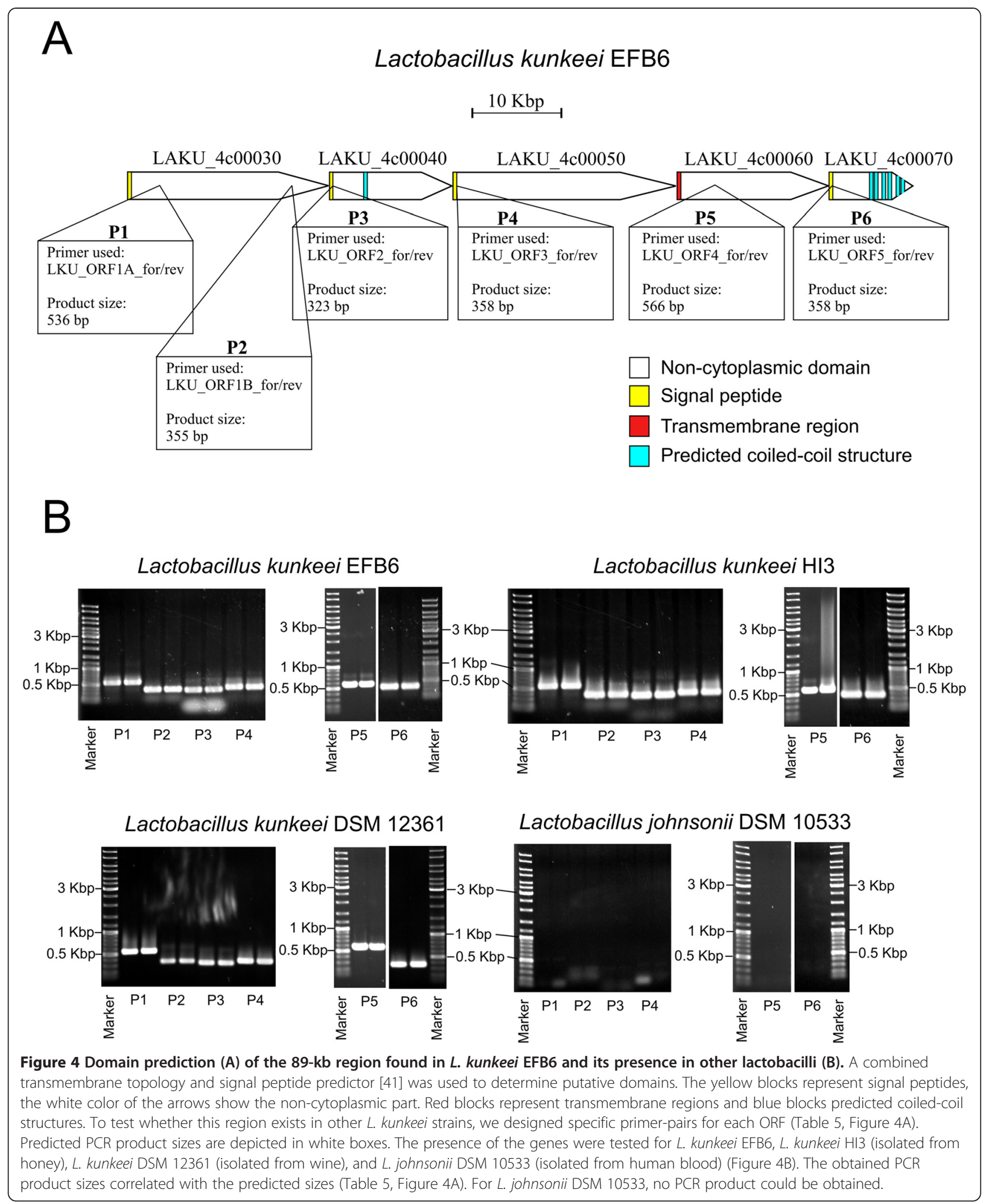

coiled coil structures. Except of LAKU_4c00050 (8,342 amino acids), all ORFs show weak similarity to large surface proteins or extracellular matrix-binding proteins found in bacteria such as Staphylococcus, Streptococcus, Burkholderia, Weissella, Mannheimia, and Marinomonas, but also in Lactobacillus and Pediococcus. Since, $L$. 
Table 5 Primer used in this study

\begin{tabular}{|c|c|c|c|}
\hline Primer & DNA sequence $\left(5^{\prime}-3^{\prime}\right)$ & Open reading frame & Product size \\
\hline LKU_ORF1A_for & AACCAAGAGTAACGATGCCC & LAKU_4C00030 & $536 \mathrm{bp}$ \\
\hline LKU_ORF1A_rev & CTITGGTAATCGGCTTGTGC & & \\
\hline LKU_ORF1B_for & CGATGCACAAACTGCTTACG & LAKU_4c00030 & $355 \mathrm{bp}$ \\
\hline LKU_ORF1B_rev & CATCCTITTGTGCGTCGTTG & & \\
\hline LKU_ORF2_for & AGCTCTITIAGGTGCGTCTG & LAKU_4c00040 & $323 \mathrm{bp}$ \\
\hline LKU_ORF2_rev & TATGCGTCTTGGTGGTTGGC & & \\
\hline LKU_ORF3_for & GCGACTITGTCTGTTITGGG & LAKU_4c00050 & $358 \mathrm{bp}$ \\
\hline LKU_ORF3_rev & ATAGCCCCAGCATATCCAGC & & \\
\hline LKU_ORF4_for & CTACGTTGAGGTTTCCGCTC & LAKU_4c00060 & $566 \mathrm{bp}$ \\
\hline LKU_ORF4_rev & GTTGGAGTTACCTTGCCACC & & \\
\hline LKU_ORF5_for & TCCCAGTAGTAACAAGTAACACC & LAKU_4c00070 & $358 \mathrm{bp}$ \\
\hline LKU_ORF5_rev & AAGCGGTTGATTTCCATTGAC & & \\
\hline
\end{tabular}

kunkeei EFB6 is the first sequenced genome harboring these cluster, we designed specific primer pairs for detection of each ORF in other Lactobacillus strains by PCR (Table 6). As shown in Figure 4B, all five ORFs were present in other L. kunkeei strains isolated from honey and wine. On the basis of domain prediction and IMG's bidirectional best hits [32], we assume that this gene cluster encodes cell surface or secreted proteins involved in cell adhesion or biofilm formation.

During genome comparison, we identified two additional proteins (LAKU_24c00010 and LAKU_24c00050) without a homolog in any of the publicly available genome sequences. These proteins show only weak sequence similarity to known proteins and might be involved in cellular adhesion. LAKU_24c00010 contains a signal peptide, transmembrane helices and 29 DUF1542 domains, which are typically found in cell surface proteins. In Staphylococcus aureus, it has been shown that some DUF1542containing proteins are involved in cellular adhesion and antibiotic resistance [42]. LAKU_24c00010 showed the highest sequence identities to the matrix-binding protein (WP_010490864) of "Lactobacillus zeae" КCTC
3804 (40\%) [43] and the extracellular matrix binding protein (YP_005866289) of Lactobacillus rhamnosus ATCC 53103 (36\%) (Figure 5).

Additionally, LAKU_24c00050 contains N terminal transmembrane helices, two mucin-binding protein domains as well as a $C$ terminal Gram positive-anchoring domain. Proteins with this domain combination are usually associated with bacterial surface proteins. LAKU_24c00050 showed similarity to the Mlp protein (WP_004239242) of Streptococcus mitis and other mucus-binding proteins (Figure 6). Due to the mucosal surface-colonizing properties of lactobacilli, they have been investigated as potential recombinant mucosal vaccines [45].

In the genome of L. kunkeei EFB6, we identified genes encoding all proteins of the general secretory (Sec) pathway and putative polysaccharide biosynthesis proteins, which may participate in capsule or S layer formation. Recently, Butler et al. (2013) [47] detected a lysozyme produced by L. kunkeei Fhon2N and suggested a bacteriolysin or class III bacteriocin function. In L. kunkeei EFB6, we identified four genes belonging to the glycoside hydrolase family 25. Enzymes of this family are known

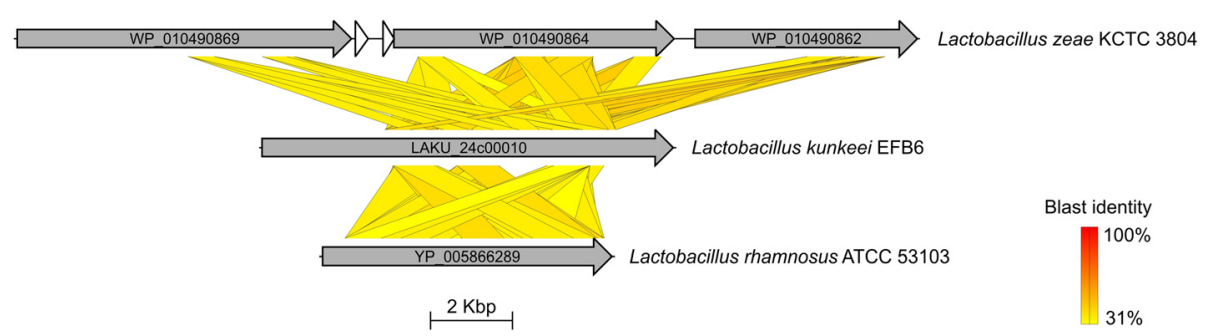

Figure 5 Tblastx comparison of $L$. kunkeei ORF LAKU_24c00010 to matrix binding proteins of L. rhamnosus ATCC 53103 and "L. zeae" KCTC 3804. The graphical presentation was done with Easyfig software (minimum blast hit length of $200 \mathrm{bp}$ and a maximum e-value of $1 \mathrm{e}^{-100}$ ) [44]. LAKU_24c00010 shows similarities to WP_010490869, WP_010490864 and WP_0104908662 of "L. zeae" KCTC 3804 , but also to YP_005866289 (L. rhamnosus ATCC 53103). The ORFs used for comparison are labeled with NCBI accession numbers. The blast identity is shown in a colored scale ranging from $31 \%$ (yellow) to $100 \%$ (red). 


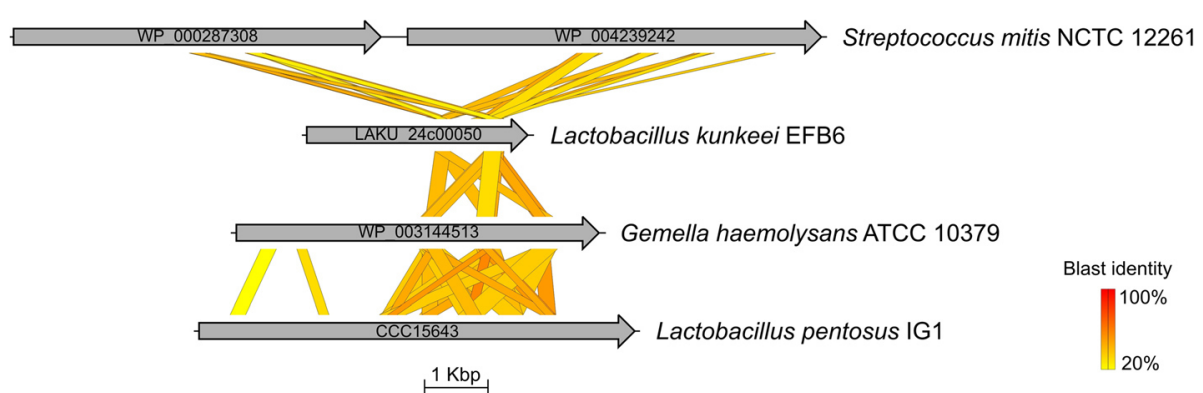

Figure 6 Tblastx comparison of MucBP domain-containing proteins. Comparison of MucBP domain-containing proteins were performed using the program Easyfig (mininum blast hit length of $50 \mathrm{bp}$ and maximum e-value of $1 \mathrm{e}^{-10}$ ) [44]. LAKU_24c00050 shows similarity to ORFs of Streptococcus mitis NCTC 12261 (NCBI accession numbers inside arrows, which represent ORFs used for comparison). Additionally, LAKU_24c00050 shows similarity to WP_003144513 of Gemella haemolysans ATCC 10379 and CCC15643 of Lactobacillus pentosus IG1 [46]. The blast identity is shown in a colored scale ranging from $20 \%$ (yellow) to $100 \%$ (red).

to possess lysozyme activity. Two of the deduced proteins (LAKU_13c00160 and LAKU_32c00010) contain a signal peptide, indicating secretion of the proteins. LAKU_19c00290 harbors transmembrane helices and is probably anchored in the cell wall. LAKU_6c00080 did not contain a putative signal peptide or transmembrane helices.

\section{Rapid test PCR}

Specific primer pairs have been designed to test other strains by PCR for the presence of an $89 \mathrm{~kb}$ region, which harbors five open reading frames (ORFs). Genomic DNA of the L. kunkeei strains EFB6, HI3 and DSM 12361, and Lactobacillus johnsonii DSM 10533 was used as template for PCR amplifications employing the thermal cycler peqSTAR 2X (PEQLAB Biotechnologie GmbH, Erlangen, Germany). PCR amplification was performed with the BIO-X-ACT ${ }^{\mathrm{Tm}}$ Short DNA Polymerase (Bioline, Luckenwalde, Germany) and an initial denaturation step at $98^{\circ} \mathrm{C}$ for $2 \mathrm{~min}$, followed by 30 cycles of denaturation for $20 \mathrm{~s}$ at $96^{\circ} \mathrm{C}$, annealing for $20 \mathrm{~s}$ at $60^{\circ} \mathrm{C}$ and elongation for $30 \mathrm{~s}$ at $68^{\circ} \mathrm{C}$. Subsequently, a final elongation step of $10 \mathrm{~min}$ at $68^{\circ} \mathrm{C}$ was performed. PCR products were purified employing the QIAquick PCR Purification Kit (Qiagen, Hilden, Germany).

\section{Conclusion}

In this study, we characterized the genome of L. kunkeei strain EFB6 isolated from an EFB-diseased larva. In a recent study was shown that $L$. kunkeei has the potential for biofilm formation and adhesion to the honey crop [6]. Our genome analysis supports these results. Using large surface proteins or extracellular matrix-binding proteins, L. kunkeei might be able to attach to eukaryotic epithelial cells. Furthermore, due to the presence of polysaccharide biosynthesis proteins and several enzymes with lysozyme activity, it is possible that $L$. kunkeei is actively protecting its niche against bacterial competitors. As
LABs have been shown to have an inhibitory growth effect on M. plutonius, the use of LABs as probiotic additive against the EFB-causing agent is conceivable.

\section{Additional file}

Additional file 1: Associated MIGS Record.

\section{Abbreviations}

AFB: American foulbrood of honeybees; EFB: European foulbrood of honeybees; LABs: Lactic acid bacteria; TEM: Transmission electron microscopy.

\section{Competing interests}

The authors declare that they have no competing interests.

\section{Authors' contributions}

$M D, A P$ and $R D$ designed research, MD, JS and FJT isolated and characterized strain EFB6, MD, AP and AL carried out genome analyses, MH performed electron microscopy, MD and RD wrote the manuscript with help of AP. All authors read and approved the final manuscript.

\section{Acknowledgements}

We thank the "Bundesministerium für Bildung und Forschung" and the "Niedersächsisches Ministerium für Wissenschaft und Kultur" for support. Additionally, we acknowledge support by the German Research Foundation and the Open Access Publication Funds of the Göttingen University.

\section{Author details}

'Department of Genomic and Applied Microbiology \& Göttingen Genomics Laboratory, Institute of Microbiology and Genetics, Georg-August University of Göttingen, Göttingen, Germany. ${ }^{2}$ Department of General Microbiology, Institute of Microbiology and Genetics, Georg-August University of Göttingen, Göttingen, Germany.

Received: 20 May 2014 Accepted: 4 December 2014 Published: 19 February 2015

\section{References}

1. Klein A-M, Vaissière BE, Cane JH, Steffan-Dewenter I, Cunningham SA, Kremen C, et al. Importance of pollinators in changing landscapes for world crops. Proc R Soc B. 2007;274:303-13.

2. Southwick EE, Southwick Jr L. Estimating the economic value of honey bees (Hymenoptera: Apidae) as agricultural pollinators in the United States. J Econ Entomol. 1992;85:621-33.

3. Forsgren E. European foulbrood in honey bees. J Invertebr Pathol. 2010;103 (Suppl):S5-9. 
4. Djukic M, Brzuszkiewicz E, Fünfhaus A, Voss J, Gollnow K, Poppinga L, et al. How to Kill the Honey Bee Larva: Genomic Potential and Virulence Mechanisms of Paenibacillus larvae. PLoS One. 2014;9:e90914.

5. Bailey L, Collins MD. Reclassification of "Streptococcus pluton" (White) in a new genus Melissococcus, as Melissococcus pluton nom. rev.; comb. nov. J Appl Microbiol. 1982;53:215-7.

6. Vásquez A, Forsgren E, Fries I, Paxton RJ, Flaberg E, Szekely L, et al. Symbionts as major modulators of insect health: lactic acid bacteria and honeybees. PLoS One. 2012;7:e33188.

7. Forsgren E, Olofsson TC, Vásquez A, Fries I. Novel lactic acid bacteria inhibiting Paenibacillus larvae in honey bee larvae. Apidologie. 2009;41:99-108.

8. Olofsson TC, Vásquez A. Detection and identification of a novel lactic acid bacterial flora within the honey stomach of the honeybee Apis mellifera. Curr Microbiol. 2008;57:356-63.

9. Endo A, Irisawa T, Futagawa-Endo Y, Takano K, du Toit M, Okada S, et al. Characterization and emended description of Lactobacillus kunkeei as a fructophilic lactic acid bacterium. Int J Syst Evol Micr. 2012;62:500-4.

10. Edwards CG, Haag KM, Collins MD, Hutson RA, Huang YC. Lactobacillus kunkeei sp. nov.: a spoilage organism associated with grape juice fermentations. J Appl Microbiol. 1998;84:698-702.

11. Woese CR, Kandler O, Wheelis ML. Towards a natural system of organisms: proposal for the domains Archaea, Bacteria, and Eucarya. Proc Natl Acad Sci. 1990:87:4576-9.

12. Gibbons NE, Murray RGE. Proposals Concerning the Higher Taxa of Bacteria. Int J Syst Bacteriol. 1978;28:1-6.

13. Garrity G, Holt J. The Road Map to the Manual. In: Bergey's Manual of Systematic Bacteriology, vol. 1. 2nd ed. New York: Springer; 2001. p. 119-69.

14. Murray R. The Higher Taxa, or, a Place for Everything...? In: Bergey's Manual of Systematic Bacteriology, vol. 1. 1st ed. Baltimore: The Williams and Wilkins Co; 1984. p. 31-4.

15. List Editor. List of new names and new combinations previously effectively, but not validly, published. List no. 132. Int J Syst Evol Microbiol. 2010;60:469-72. doi: 10.1099/ijs.0.022855-0.

16. Ludwig W, Schleifer K, Whitman W. Class I. Bacilli class nov. In: Bergey's Manual of Systematic Bacteriology, Second Edition, Volume 3. New York: Springer; 2009. p. 19-20.

17. Schleifer K, Whitman W. Order II. Lactobacillales ord. nov. In: Bergey's Manual of Systematic Bacteriology, Second Edition, Volume 3 (The Firmicutes). New York: Springer; 2009. p. 464.

18. Skerman V, McGowan V, Sneath P. Approved Lists of Bacterial Names. Int J Syst Bacteriol. 1980;30:225-420.

19. Haakensen M, Dobson C, Hill J, Ziola B. Reclassification of Pediococcus dextrinicus (Coster and White 1964) Back 1978 (Approved Lists 1980) as Lactobacillus dextrinicus comb. nov., and emended description of the genus Lactobacillus. Int J Syst Evol Micr. 2009;59:615-21.

20. Cai Y, Pang H, Kitahara M, Ohkuma M. Lactobacillus nasuensis sp. nov., a lactic acid bacterium isolated from silage, and emended description of the genus Lactobacillus. Int J Syst Evol Micr. 2012;62:1140-4

21. Bergey DH, Breed RS, Hammer BW, Huntoon FM, Murray EGD, Harrison FC. Genus X. Lactobacillus Beijerinck, 191. In: Bergey DH, Breed RS, Hammer BW, Huntoon FM, Murray EGD, Harrison FC, editors. Bergey's Manual of Systematic Bacteriology. 4th ed. Baltimore: The Williams and Wilkins Co; 1934. p. 300-21.

22. Bundesanstalt für Arbeitsschutz und Arbeitsmedizin. Technical rules for biological agents. TRBA 466. Available at: http://www.baua.de/de/ Themen-von-A-Z/Biologische-Arbeitsstoffe/TRBA/TRBA-466.html.

23. Hoppert M. Microscopic Techniques in Biotechnology. Weinheim, FRG: Wiley-VCH Verlag GmbH \& Co. KGaA; 2003. Available at: http://doi.wiley. com/10.1002/3527602615.

24. Mayer F. Cytology and Morphogenesis of Bacteria (Handbook of plant Anatomy IV, 2). Stuttgart: Borntraeger; 1988.

25. Thompson JD, Higgins DG, Gibson TJ. CLUSTAL W: improving the sensitivity of progressive multiple sequence alignment through sequence weighting, position-specific gap penalties and weight matrix choice. Nucleic Acids Res. 1994:22:4673-80.

26. Kumar S, Nei M, Dudley J, Tamura K. MEGA: a biologist-centric software for evolutionary analysis of DNA and protein sequences. Brief Bioinform. 2008;9:299-306.

27. Bankevich A, Nurk S, Antipov D, Gurevich AA, Dvorkin M, Kulikov AS, et al. SPAdes: a new genome assembly algorithm and its applications to single-cell sequencing. J Comput Biol. 2012;19:455-77.
28. Tech M, Merkl R. YACOP: Enhanced gene prediction obtained by a combination of existing methods. In Silico Biol. 2003;3:441-51.

29. Delcher AL, Harmon D, Kasif S, White O, Salzberg SL. Improved microbial gene identification with GLIMMER. Nucleic Acids Res. 1999;27:4636-41.

30. Lagesen $\mathrm{K}$, Hallin P, Rødland EA, Staerfeldt H-H, Rognes T, Ussery DW. RNAmmer: consistent and rapid annotation of ribosomal RNA genes. Nucleic Acids Res. 2007;35:3100-8.

31. Lowe TM, Eddy SR. tRNAscan-SE: a program for improved detection of transfer RNA genes in genomic sequence. Nucleic Acids Res. 1997;25:955-64.

32. Markowitz VM, Mavromatis K, Ivanova NN, Chen I-MA, Chu K, Kyrpides NC. IMG ER: a system for microbial genome annotation expert review and curation. Bioinformatics. 2009;25:2271-8

33. Gasteiger E, Jung E, Bairoch A. SWISS-PROT: connecting biomolecular knowledge via a protein database. Curr Issues Mol Biol. 2001;3:47-55.

34. O'Donovan C, Martin MJ, Gattiker A, Gasteiger E, Bairoch A, Apweiler R. High-quality protein knowledge resource: SWISS-PROT and TrEMBL. Brief Bioinform. 2002;3:275-84.

35. Hunter S, Jones P, Mitchell A, Apweiler R, Attwood TK, Bateman A, et al. InterPro in 2011: new developments in the family and domain prediction database. Nucleic Acids Res. 2012;40:D306-12.

36. Tatusov RL, Fedorova ND, Jackson JD, Jacobs AR, Kiryutin B, Koonin EV, et al. The COG database: an updated version includes eukaryotes. BMC Bioinformatics. 2003;4:41.

37. Altschul SF, Gish W, Miller W, Myers EW, Lipman DJ. Basic local alignment search tool. J Mol Biol. 1990;215:403-10.

38. Alikhan NF, Petty NK, Ben Zakour NL, Beatson SA. BLAST Ring Image Generator (BRIG): simple prokaryote genome comparisons. BMC Genomics. 2011;12:402.

39. Lechner M, Findeiss S, Steiner L, Marz M, Stadler PF, Prohaska SJ. Proteinortho: detection of (co-)orthologs in large-scale analysis. BMC Bioinformatics. 2011;12:124.

40. Leimbach A. bac-genomics-scripts. 2014. Available at: https://github.com/ aleimba/bac-genomics-scripts.

41. Käll L, Krogh A, Sonnhammer EL. A combined transmembrane topology and signal peptide prediction method. J Mol Biol. 2004;338:1027-36.

42. Clarke SR, Harris LG, Richards RG, Foster SJ. Analysis of Ebh, a 1.1megadalton cell wall-associated fibronectin-binding protein of Staphylococcus aureus. Infect Immun. 2002;70:6680-7.

43. Dicks LMT, Du Plessis EM, Dellaglio F, Lauer E. Reclassification of Lactobacillus casei subsp. casei ATCC 393 and Lactobacillus rhamnosus ATCC 15820 as Lactobacillus zeae nom. rev., designation of ATCC 334 as the neotype of $L$. casei subsp. casei, and rejection of the name Lactobacillus paracasei. Int J Syst Bacteriol. 1996;46:337-40.

44. Sullivan MJ, Petty NK, Beatson SA. Easyfig: a genome comparison visualizer. Bioinformatics. 2011;27:1009-10.

45. Turner MS, Hafner LM, Walsh T, Giffard PM. Peptide surface display and secretion using two LPXTG-containing surface proteins from Lactobacillus fermentum BR11. Appl Environ Microbiol. 2003:69:5855-63.

46. Zanoni P, Farrow JAE, Phillips BA, Collins MD. Lactobacillus pentosus, (Fred, Peterson and Anderson) sp. nov., nom. rev. Int J Syst Bacteriol. 1987;37:339-41.

47. Butler E, Alsterfjord M, Olofsson TC, Karlsson C, Malmström J, Vásquez A. Proteins of novel lactic acid bacteria from Apis mellifera mellifera: an insight into the production of known extra-cellular proteins during microbial stress. BMC Microbiol. 2013;13:235.

doi:10.1186/1944-3277-10-16

Cite this article as: Djukic et al:: High quality draft genome of

Lactobacillus kunkeei EFB6, isolated from a German European foulbrood outbreak of honeybees. Standards in Genomic Sciences 2015 10:16. 


\title{
7.1 Ergänzende Daten zu Kapitel B7
}

\author{
Inhaltsverzeichnis
}

Table S1. Associated MIGS Record.

Die Tabelle S1 ist auf dem beigefügten, digitalen Medium verfügbar. Dazu bitte den Ordner „Kapitel_B7_Ergänzende_Daten“ öffnen. 


\title{
First Insights into the Genome of Fructobacillus sp. EFB-N1, Isolated from Honey Bee Larva Infected with European Foulbrood
}

\author{
Marvin Djukic ${ }^{1}$, Rolf Daniel ${ }^{1}$ und Anja Poehlein ${ }^{1}$ \\ Genome Announcements (2015), Vol. 3 (4), e00868-15 \\ ${ }^{1}$ Abteilung für Genomische und Angewandte Mikrobiologie \& Göttingen Genomics \\ Laboratory, Institut für Mikrobiologie und Genetik, Georg-August Universität Göttingen, \\ Göttingen, Deutschland
}

\section{Anteilserklärung}

Idee/Konzept: MD, AP, RD

Genomsequenzierung: AP

In silico Genomanalyse: MD

Schreiben des Manuskripts: MD, AP, RD 


\title{
First Insights into the Genome of Fructobacillus sp. EFB-N1, Isolated from Honey Bee Larva Infected with European Foulbrood
}

\author{
Marvin Djukic, Rolf Daniel, Anja Poehlein \\ Genomic and Applied Microbiology and Göttingen Genomics Laboratory, Institute of Microbiology and Genetics, Georg-August University Göttingen, Göttingen, \\ Germany
}

European foulbrood is a worldwide disease affecting the honey bee brood. Here, we report the draft genome sequence of Fructobacillus sp. EFB-N1, which was isolated from an infected honey bee larva derived from a Swiss European foulbrood outbreak. The genome consists of 68 contigs and harbors 1,629 predicted protein-encoding genes.

Received 24 June 2015 Accepted 24 June 2015 Published 30 July 2015

Citation Djukic M, Daniel R, Poehlein A. 2015. First insights into the genome of Fructobacillus sp. EFB-N1, isolated from honey bee larva infected with European foulbrood. Genome Announc 3(4):e00868-15. doi:10.1128/genomeA.00868-15

Copyright $\odot 2015$ Djukic et al. This is an open-access article distributed under the terms of the Creative Commons Attribution 3.0 Unported license.

Address correspondence to Rolf Daniel, rdaniel@gwdg.de.

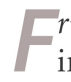
ructobacillus sp. EFB-N1 was isolated from a honey bee larva infected with European foulbrood (EFB). The nonmotile isolate grows aerobically on de Man/Rogosa/Sharpe (MRS) agar (1) at $32^{\circ} \mathrm{C}$. The $16 \mathrm{~S}$ rRNA gene sequence comparison showed a $99.7 \%$ DNA sequence identity to Fructobacillus tropaeoli F214-1, which is a fructophilic lactic acid bacterium isolated from a flower (2). Comparison of recA gene sequences is used as a marker for classification of Fructobacillus species $(3,4)$. The recA gene sequence of strain EFB-N1 revealed 91\% DNA sequence similarity to the corresponding gene of F. tropaeoli.

Chromosomal DNA of Fructobacillus sp. EFB-N1 was isolated with the MasterPure complete DNA purification kit as recommended by the manufacturer (Epicentre, Madison, WI, USA). The extracted DNA was used to generate Illumina shotgun libraries, which were subsequently sequenced with a Genome Analyzer IIx as recommended by the manufacturer (Illumina, San Diego, CA, USA). Trimming and removal of low-quality reads with Trim Galore version 0.4.0 (http://www .bioinformatics.babraham.ac.uk/projects/trim_galore) resulted in 5,374,914 paired-end Illumina reads. Genome assembly was performed with SPAdes software version 3.5.0 (5) and yielded 68 contigs ( $>500 \mathrm{bp}$ ) and 368 -fold coverage. The draft genome of Fructobacillus sp. EFB-N1 comprises $1.64 \mathrm{Mbp}$, with an overall $\mathrm{G}+\mathrm{C}$ content of $43.7 \%$. Open reading frame (ORF) and RNA detection were verified with Prodigal version 2.6.2 (6) and Barrnap version 0.6 (http://www.vicbioinformatics.com /software.barrnap.shtml), respectively. Annotation was performed with Prokka version 1.11 (7). The draft genome harbored one rRNA cluster, 46 tRNA genes, 1,210 predicted protein-coding genes with function assignment, and 419 putative genes coding for hypothetical proteins.

Genes coding for a putative bacterial conjugation machinery were located on contig FEFB_c000008. In addition, potential genes encoding proteins involved in adhesion and biofilm formation that showed high amino acid sequence similarity to corresponding predicted proteins encoded by the genomes of other
Fructobacilli were identified. Noteworthy, the genome of Fructobacillus sp. EFB-N1 lacks a complete glycolysis system and pentose phosphate pathway, as putative genes encoding phosphofructokinase, fructose-bisphosphate aldolase, transketolase, and transaldolase were missing. Additionally, strain EFB-N1 completely lacked genes coding for citrate cycle enzymes. A putative lactate dehydrogenase-encoding gene as part of a homolactic fermentation pathway is present. In addition, the presence of a putative sucrase-encoding gene indicated the ability to convert sucrose to fructose and glucose.

Nucleotide sequence accession numbers. This whole-genome shotgun project has been deposited at DDBJ/EMBL/GenBank under the accession number LDUY00000000. The version described in this paper is the first version, LDUY01000000.

\section{ACKNOWLEDGMENTS}

We thank the "Bundesministerium für Bildung und Forschung" and the "Niedersächsisches Ministerium für Wissenschaft und Kultur" for support.

\section{REFERENCES}

1. De Man JC, Rogosa M, Sharpe ME. 1960. A medium for the cultivation of lactobacilli. J Appl Bacteriol 23:130-135. http://dx.doi.org/10.1111/j.1365 -2672.1960.tb00188.x.

2. Endo A, Irisawa T, Futagawa-Endo Y, Sonomoto K, Itoh K, Takano K, Okada S, Dicks LM. 2011. Fructobacillus tropaeoli sp. nov., a fructophilic lactic acid bacterium isolated from a flower. Int J Syst Evol Microbiol 61: 898-902. http://dx.doi.org/10.1099/ijs.0.023838-0.

3. Torriani S, Felis GE, Dellaglio F. 2001. Differentiation of Lactobacillus plantarum, $L$. pentosus, and $L$. paraplantarum by recA gene sequence analysis and multiplex PCR assay with recA gene-derived primers. Appl Environ Microbiol 67:3450-3454. http://dx.doi.org/10.1128/AEM.67.8.3450 -3454.2001 .

4. Endo A, Okada S. 2008. Reclassification of the genus Leuconostoc and proposals of Fructobacillus fructosus gen. nov., comb. nov., Fructobacillus durionis comb. nov., Fructobacillus ficulneus comb. nov. and Fructobacillus pseudoficulneus comb. nov. Int J Syst Evol Microbiol 58:2195-2205. http:// dx.doi.org/10.1099/ijs.0.65609-0. 
5. Bankevich A, Nurk S, Antipov D, Gurevich AA, Dvorkin M, Kulikov AS, Lesin VM, Nikolenko SI, Pham S, Prjibelski AD, Pyshkin AV, Sirotkin AV, Vyahhi N, Tesler G, Alekseyev MA, Pevzner PA. 2012. SPAdes: a new genome assembly algorithm and its applications to single-cell sequencing. J Comput Biol 19:455-477. http://dx.doi.org/ 10.1089/cmb.2012.0021.
6. Hyatt D, Chen G-L, Locascio PF, Land ML, Larimer FW, Hauser LJ. 2010. Prodigal: prokaryotic gene recognition and translation initiation site identification. BMC Bioinformatics 11:119. http://dx.doi.org/10.1186/ 1471-2105-11-119.

7. Seemann T. 2014. Prokka: rapid prokaryotic genome annotation. Bioinformatics 30:2068-2069. http://dx.doi.org/10.1093/bioinformatics/btu153. 


\section{Diskussion}

Honigbienen besitzen weltweit sowohl ökonomisch wie auch ökologisch einen sehr hohen Stellenwert (Klein et al., 2007; Steffan-Dewenter et al., 2005). Daher beschäftigten sich die Menschen bereits sehr früh mit Bienenkrankheiten. Bereits im antiken Griechenland schrieb Aristoteles (384 - 322 vor Christus) in seinem Werk „Historia Animalium IX“ seine Gedanken und Forschungen über die Krankheiten der Biene nieder. Die erste Namensgebung „Faulbrut“ erfolgte von Schirach im Jahr 1769 (Genersch, 2008). Ungefähr ein Jahrhundert später beschrieb Dzierzon zwei verschiedene Krankheiten der Honigbienenbrut - die eine ,mild und behandelbar' und die andere ,bösartig und nicht behandelbar“ (Dzierzon, 1882). 1885 wurde das Bakterium „Bacillus alvei“ als Erreger der Faulbrut ermittelt (Cheshire \& Cheyne, 1885), jedoch gelang es White 21 Jahre später nicht, den Erreger aus Faulbrut-verstorbenen Larven isolieren (Genersch, 2008; White, 1906). Stattdessen gab er einem anderen, von ihm aus der Faulbrut isolierten Bakterium den Namen „Bacillus larvae“ (White, 1906). Von diesem Zeitpunkt an war klar, dass es zwei verschiedene, bakteriell verursachte Bienenbrutkrankheiten mit demselben Namen „Faulbrut“" gab. Zum einen die EFB, welche durch M. plutonius verursacht und von „Bacillus alvei“ als Sekundärerreger regelmäßig begleitet wird (Bailey, 1983), und zum anderen die AFB mit „Bacillus larvae“ als auslösenden Erreger (White, 1906). Die Speziesbezeichnungen wurden im Laufe der Zeit aktualisiert, wenngleich diese historische Ausführung zeigen soll, dass beide Krankheiten bereits seit langer Zeit bekannt und Ziel von Forschungsaktivitäten sind. Die exakte Pathogenese beider Krankheiten ist noch nicht aufgeklärt, allerdings kann man sagen, dass die AFB-bezogene Forschung der EFBForschung bei weitem vorauseilt, da bis zu dieser Studie kein einziger Virulenzfaktor von M. plutonius bekannt war. In den folgenden Abschnitten werden die ermittelten Daten aus der Genomanalyse der Erreger in den Kontext der jeweiligen Pathogenese gestellt.

\section{Unterschiede und Gemeinsamkeiten zwischen AFB und EFB: Funktionelle und vergleichende Genomanalyse von $P$. larvae (AFB) und M. plutonius (EFB)}

Sowohl P. larvae als auch M. plutonius lösen eine intestinale Infektion bei der Honigbienenlarve aus. Ein vergleichendes Infektions- und Pathogenesemodell ist in Abbildung 7 dargestellt. 


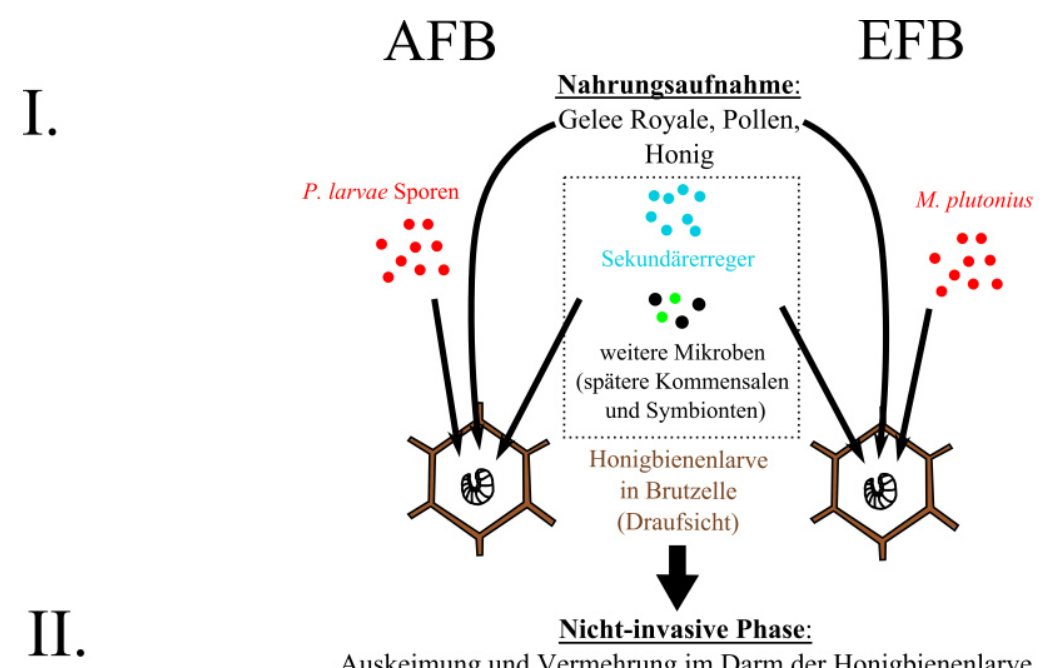

Auskeimung und Vermehrung im Darm der Honigbienenlarve

Konkurrenzkampf im Darm mit mikrobiellen Kommensalen und Symbionten Verdrängung aller Kommensalen, Symbionten und Sekundärerreger ( $P$. larvae)

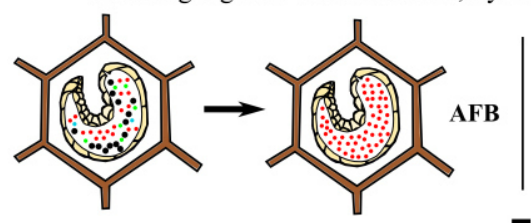

EFB
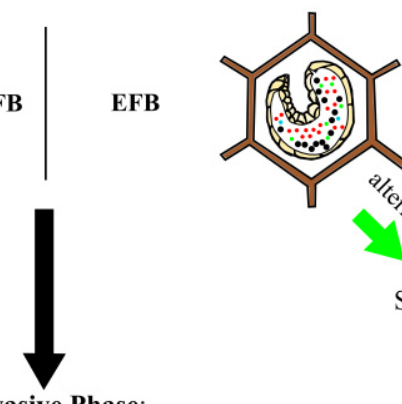

arve oder Puppe überleben EFB-Infektion. Schlüpfende Bienen sind oftmals kleinwüchsig

III.

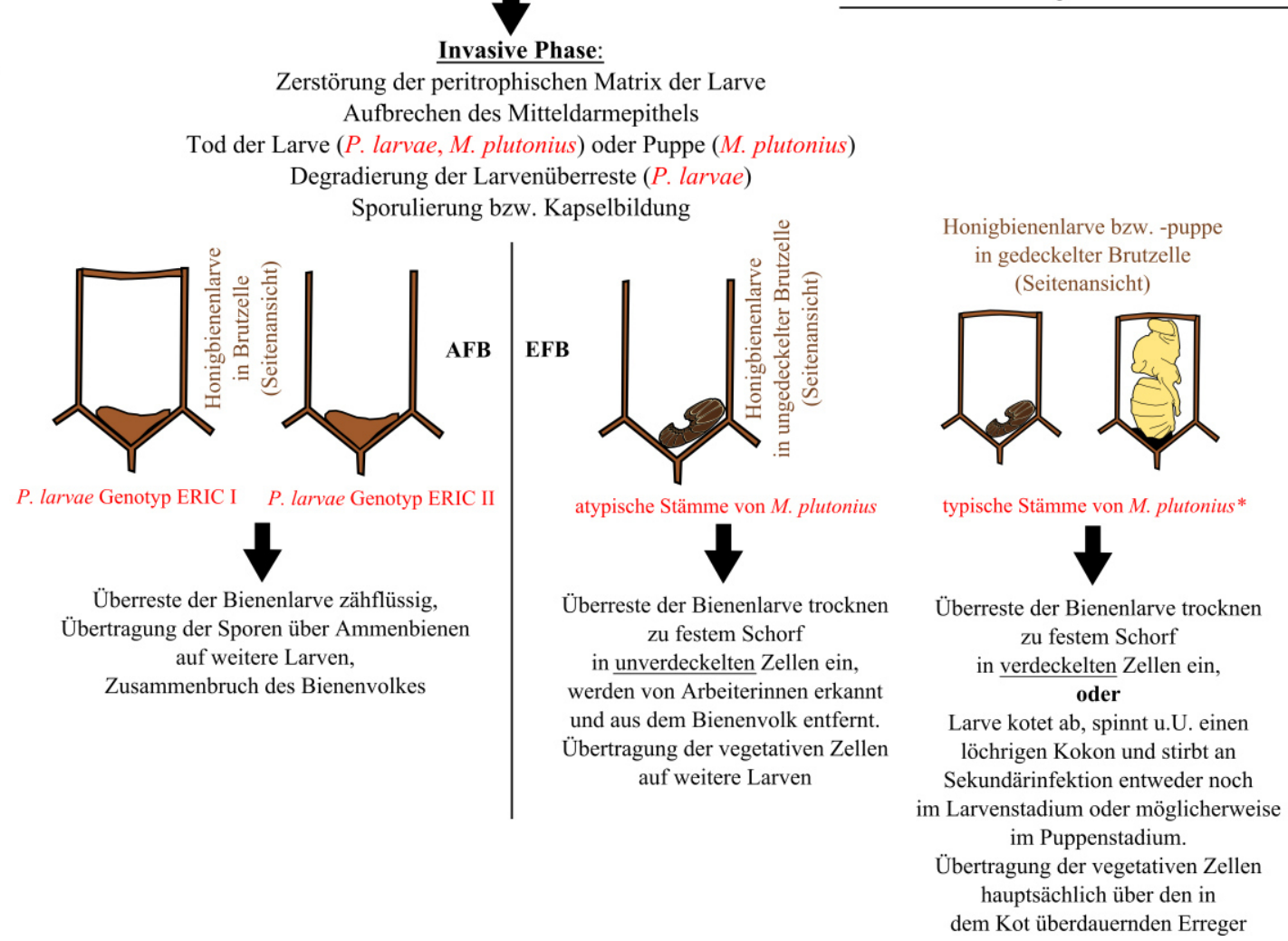

Abbildung 7: AFB und EFB-Infektionsmodell.

Die Erstellung des AFB-Infektions- und Pathogenesemodells beruht auf Kapitel B2 und Genersch \& Poppinga (2015). Das EFB-Modell beruht auf Kapitel B4.

Beide Modelle können in Nahrungsaufnahme (I), nicht-invasive Phase (II) und invasive Phase (III) eingeteilt werden. Obwohl normalerweise nur Larven für AFB und EFB-Infektionen anfällig sind, führt der Mangel an Nachwuchs letztlich zum Kollaps des Bienenvolkes. Zu beachten ist beim 
EFB-Infektionsmodell, dass Larven, die bereits eine EFB-Infektion überlebt haben, oftmals nur einen ungenügenden Schutz (löchriger Kokon) ausbilden und somit für Sekundärinfektionen anfällig sind (Ritter, 2012). Eine Sekundärinfektion muss dabei nicht zwangsläufig von $M$. plutonius ausgehen. Die Markierung (Stern) bedeutet, dass nicht jede Infektion mit einem typischen Stamm (M. plutonius) zum Tod der Larve oder Puppe führen muss.

\subsection{Nahrungsaufnahme und Nicht-invasive Phase der AFB- und EFB-Pathogenese}

\subsection{1 Überdauerungsformen bei $P$. larvae und $M$. plutonius}

Über die Aufnahme von kontaminierter Nahrung gelangen infektiöse Sporen von P. larvae oder vegetative Zellen von M. plutonius in den Darm der Larve (Forsgren, 2010; Genersch, 2010b). Sowohl Endosporen als auch Kapseln gelten als Überdauerungsformen bei Bakterien. Sie bieten in der Regel Schutz gegen Austrocknung und Hitze, aber auch gegen toxische oder andere schädigende Agenzien (Cross, 1990; Nicholson et al., 2002). Die Sporenbildung ist bei $P$. larvae ein essentieller Vorgang und wird als letzter Schritt der AFB Pathogenese angesehen. Zugleich sind es die P. larvae-Sporen, die zu einer Infektion führen und somit den Beginn der Pathogenese einläuten (Abbildung 7) (Genersch, 2010a). Durch die Genomanalyse von P. larvae wurden Gene ermittelt, die zu eben dieser Sporenbildung führen. Im Vergleich dazu wird M. plutonius als kapselbildendes Bakterium beschrieben (Genersch, 2010b; Ritter, 2012). Auch eine Kapsel kann als Virulenzfaktor angesehen werden, da diese einigen invasiven Mikroben erlaubt, vom Immunsystem des Wirtsorganismus nicht erkannt zu werden (Graveline et al., 2007; Peterson et al., 1978; Wessels et al., 1991). In allen in dieser Arbeit sequenzierten M. plutonius-Genomen wurden Nonsens- oder Frameshift-Mutationen in dem Kapsel-kodierenden Gencluster (cps-Lokus) ermittelt. Das ermittelte Cluster besteht bei M. plutonius aus 15 Genen und weist auffällige DNA-Sequenzähnlichkeiten zu Kapsel-kodierenden Genclustern von verschiedenen Enterococci auf (Abbildung 8). 


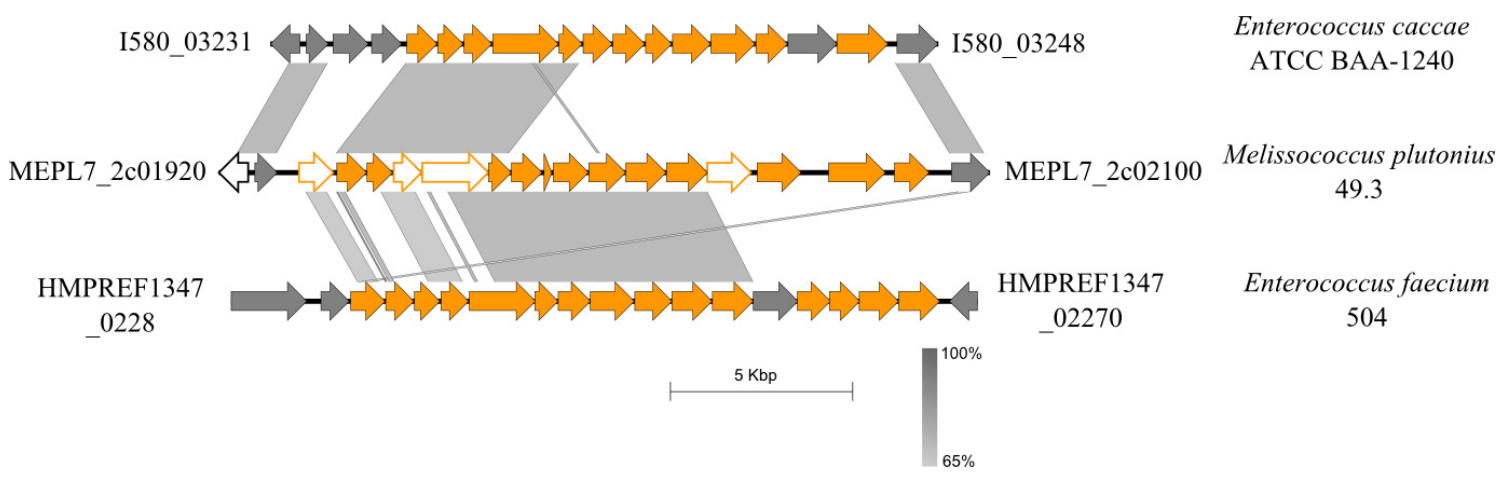

Abbildung 8: Vergleich der für die Kapselbildung kodierenden Genclustern von verschiedenen Enterococci mit dem entsprechenden Gencluster aus M. plutonius 49.3.

Die jeweiligen Enden der dargestellten Genomsegmente sind mit Locus-tags versehen. ORFs, die potentielle Kapselproteine kodieren, sind in orange hervorgehoben. ORFs, die konservierte, hypothetische Proteine kodieren, sind in grau markiert. Nicht ausgefüllte ORFs stellen Pseudogene dar.

Bei E. faecalis konnte gezeigt werden, dass nicht alle Gene des cps-Lokus für eine Kapselbildung benötigt werden (Chowdhury et al., 2014; Hancock and Gilmore, 2002; Thurlow et al., 2009). Auch ist bekannt, dass der cps-Lokus von E. faecium starke Variationen aufweist (Palmer et al., 2012). Die Vermutung liegt nahe, dass möglicherweise auch bei M. plutonius nicht alle Gene dieses Lokus für eine Kapselbildung notwendig sind. Welche Gene allerdings essentiell und welche nicht essentiell sind, kann anhand der vorliegenden Daten und einer vergleichenden Genomanalyse nicht abgeleitet werden. Die Überdauerungsform würde einen Vorteil darstellen, da diese Schutz vor Austrocknung im Kot der Larve bieten könnte.

\subsubsection{Konkurrenzkampf im Darm der Honigbienenlarve}

Im Darm der Honigbienenlarve keimt $P$. larvae aus und geht in das nicht-invasive, kommensalen-artige Stadium über (Poppinga \& Genersch, 2015). Dabei vermehrt sich $P$. larvae massiv, bis annähernd der gesamte Darm ausgefüllt ist (Abbildung 7). P. larvae ist im Gegensatz zu M. plutonius in der Lage, sich gegen bakterielle Kommensalen, Symbionten und Sekundärerreger (auch Pilze) durchzusetzen. Durch die vergleichende Genomanalyse der Stämme DSM 25719 (Genotyp ERIC I) und DSM 25430 (Genotyp ERIC II) wurden diverse NRPS und NRPS/PKS Hybridgencluster nachgewiesen, die in $M$. plutonius nicht vorkommen (Kapitel B2, für eine aktualisierte Fassung siehe Abbildung 9). Weiterführende Analysen haben gezeigt, dass drei der vier Gencluster Sekundärmetabolite kodieren, die gegen Bakterien und Pilze wirken (Kapitel B2 und B3) (Garcia-Gonzalez, et al., 2014a, 2014b). 
A)

ERIC I and II:

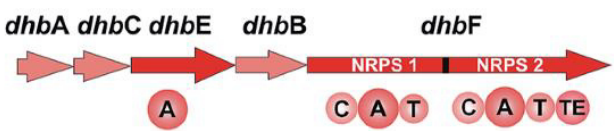

C)

ERIC II:
B)
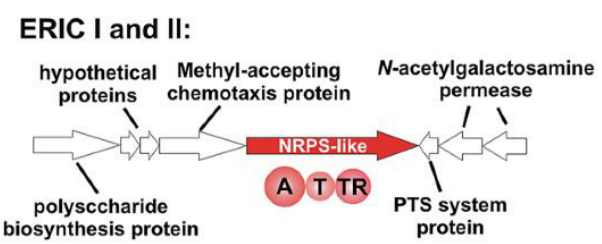

AT AT KS CTA CTA CAT C ATE CATE CAT CATCATE CATTE

ERIC I:

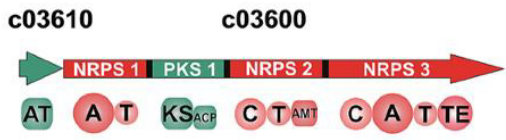

D)

ERIC II:

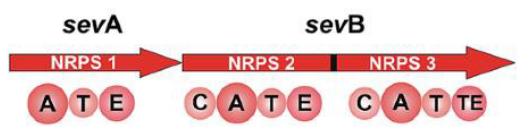

ERIC I:

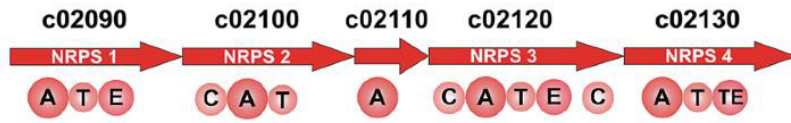

E)

ERIC I and II:

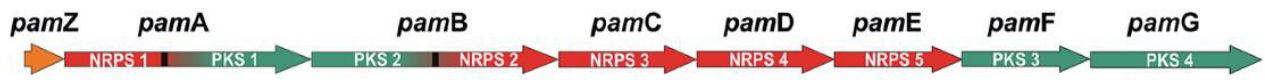

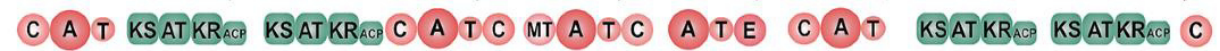

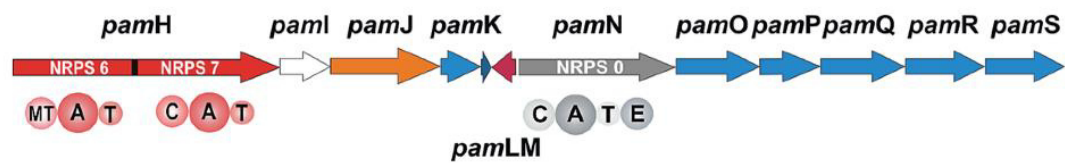

Abbildung 9: Übersicht über die in P. larvae (ERIC I und II) ermittelten NRPS und NRPS/PKS Hybridgencluster (Kapitel B2). Die Abbildung stammt aus Müller et al., 2015.

Das Bacillibactin Gencluster ist in A dargestellt. In B erkennt man ein bislang nicht näher charakterisiertes, NRPS-ähnliches Gencluster. Das Lipopeptid Gencluster von ERIC II ist zuständig für die Biosynthese von Paenilarvinen (C). Genotyp ERIC I kodiert nur einen Teil des Genclusters, dessen Sekundärmetabolit bislang unbekannt ist. D zeigt das für die SevadicinBiosynthese zuständige Gencluster von ERIC II. Das dazugehörige, leicht größere Gencluster von ERIC I wurde noch nicht näher erforscht. Das in ERIC I und II vorkommende, für PaenilamicinBiosynthese verantwortliche Gencluster ist in E abgebildet. Gene, die für die VorläuferproteinBiosynthese zuständig sind, wurden in blau markiert, Gene die für Resistenz-/Transportproteine kodieren in orange, Gene für die transkriptionelle Regulation in rot und Gene, die für Proteine mit bislang unbekannter Funktion kodieren, in weiß. Domänen wurden unterhalb des entsprechenden Gens aufgelistet: C: Kondensationsdomäne; A: Adenylierungsdomäne; T: Thiolierungsdomäne; TE: Thioesterasedomäne; TR: Thioesterase-Reduktase-Domäne; AMT: Klasse III Animotransferasedomäne; E: Epimerisierungsdomäne; MT: Methylierungsdomäne; KS: Ketosynthase-Domäne; AT: Acyltransferase Domäne; KR: Ketoreduktase Domäne; ACP: AcylTrägerprotein. 
Sevadicine zeigen antibakterielle und Paenilarvine antimykotische Aktivität (GarciaGonzalez et al., 2014a) (Kapitel B3). Dagegen zeigen Paenilamicine mit antibakterieller und antimykotischer Aktivität ein breites Wirkungsspektrum (Garcia-Gonzalez et al., 2014b). Mit Hilfe der Paenilarvine und Paenilamicine kann P. larvae sein Habitat gegen Nahrungskonkurrenten verteidigen. Zusätzlich besitzen Paenilarvine und Paenilamicine auch zytotoxische Aktivität. Dennoch zeigen Paenilamicin-Deletionsmutanten keinen Einfluss auf die Larvensterblichkeit (Garcia-Gonzalez et al., 2014b; Poppinga \& Genersch, 2015). Ein weiteres NRPS-Gencluster bei P. larvae (ERIC I und II) ist für die Biosynthese eines Bacillibactin- bzw. Paenibactin-artigen Siderophors zuständig (Kapitel B2) (Hertlein et al., 2014). Siderophore sollen die Eisenversorgung der Zelle in einer eisenarmen Umgebung sicherstellen (Lee et al., 2011). Wirtsorganismus und pathogene Organismen stehen in einem ständigen Konkurrenzkampf und für die meisten pathogenen Bakterien ist Eisen ein limitierender Faktor (Müller et al., 2015). Wenngleich die Rolle dieses Siderophors bei der AFB-Pathogenese ungeklärt ist und Siderophore nicht immer als Virulenzfaktoren angesehen werden (Müller et al., 2015), stellt es einen Fitnessfaktor für den Träger dar. Neben der Bindung und Aufnahme von Eisenionen über Siderophore sind auch weitere spezialisierte Eisentransportproteine von Bedeutung (Krewulak \& Vogel, 2008). Dazu gehören das ABC Transportsystem FeuABC und FeoAB (Cartron et al., 2006; Miethke et al., 2006), deren Gencluster nur bei P. larvae und dem atypischen $M$. plutonius Stamm DAT561 vollständig vorliegen.

Im Gegensatz zu den nicht-ribosomal gebildeten Peptiden werden Bacteriocine am Ribosom erzeugt. Der Konkurrenzkampf im Darm der Honigbienenlarve erfolgt sowohl bei der EFB als auch bei der AFB über die Bildung dieser antimikrobiellen Peptide. Bacteriocine sind abundant und besitzen eine große Diversität (Yang et al., 2014). Laut Klaenhammer besitzen 99 Prozent aller Bakterien die genetische Ausstattung um zumindest ein Bacteriocin zu bilden (Klaenhammer, 1988). Im Genom von P. larvae DSM 25719 (ERIC I) wurden drei und im Genom von DSM 25430 (ERIC II) zwei BacteriocinBiosynthese-Gencluster identifiziert, die Ähnlichkeiten zu Lantibiotika besitzen (Kapitel B2). Lantibiotika sind Bacteriocine, die Aminosäuren wie Lanthionin oder BMethyllanthionin aber auch dehydratisierte Aminosäuren enthalten (Guder et al., 2000). Neuere Analysen zeigen sogar neun potentielle Bacteriocin-kodierende Gene oder Gencluster bei DSM 25430 (ERIC II) (Müller et al., 2015), wenngleich die Funktionalität der meisten Gene und Gencluster aufgrund von Nonsens-Mutationen und inserierten Transposasen unklar ist. Bei M. plutonius wurden sieben (typische Stämme) bzw. fünf 
(atypischer Stamm) Gene bzw. Gencluster ermittelt, die für Bacteriocine und BacteriocinTransportproteine kodieren (Kapitel B4). Die Funktionalität der Gene bzw. Gencluster ist bisher unklar, da die Mehrzahl der Gencluster Mutationen enthält, die zu Pseudogenen führten.

Zusammenfassend kann man sagen, dass $M$. plutonius durch das vollständige Fehlen dieser NRPS und NRPS/PKS Hybridgencluster sowohl im Konkurrenzkampf gegen kompetitive Bakterien und Pilze im Vergleich zu P. larvae im Nachteil ist und diesen auch nicht durch die Bildung von Bacteriocinen kompensieren kann. Des Weiteren kann von einer verminderten Eisenaufnahmefähigkeit der typischen $M$. plutonius Stämme ausgegangen werden, weil entsprechende Gene als Pseudogene vorliegen.

\subsubsection{Energie und Zuckermetabolismus}

Sowohl Energiegewinnung als auch der Zuckerstoffwechsel eines Organismus können an das jeweilige Habitat angepasst sein. Die Honigbienenlarve wird mit einer sehr nährstoffhaltigen Zuckerlösung gefüttert, die auch Pollen enthalten kann (Kapitel B4). Damit sich Pathogene in diesem Habitat gegen Nahrungskonkurrenten durchsetzen können, ist neben der Betrachtung von Virulenzfaktoren auch die Analyse von Fitnessfaktoren notwendig.

Der Energiemetabolismus von $P$. larvae und $M$. plutonius unterscheiden sich grundlegend. Während P. larvae als ein fakultativ anaerobes Bakterium beschrieben ist (Heyndrickx et al., 1996), wachsen typische M. plutonius Stämme ausschließlich unter mikroaerophilen bis anaeroben Bedingungen mit ungefähr $5 \mathrm{Vol} .-\% \mathrm{CO}_{2}$. Lediglich atypische M. plutonius Stämme sind in der Lage unter aeroben Bedingungen zu wachsen. Über die Genomanalyse von P. larvae konnten alle Enzyme der Atmungskette ermittelt werden (Kapitel B2). Zusätzlich ist $P$. larvae in der Lage, Nitrat als alternativen Elektronenakzeptor zu benutzen. Allerdings enthält nur der Stamm DSM 25430 (ERIC II) neben Genen für die Nitratreduktase auch Gene für eine Nitritreduktase. Dagegen besitzt $M$. plutonius keinen vollständigen Citratzyklus und auch kein Elektronentransportsystem für oxidative Phosphorylierung (Okumura et al., 2011). Stattdessen erfolgt die Energiegewinnung über Aminosäuredecarboxylierung und über den Arginin-Deiminase-Weg (Kapitel B4) (Schimke et al., 1966).

Sowohl im Genom von $P$. larvae als auch in dem von $M$. plutonius sind alle Gene für die Glykolyse und den Pentose-Phosphat-Wegs vorhanden (Kapitel B2 und B4). Wie in Abbildung $\quad 10$ dargestellt, unterscheiden sich allerdings die 
Zuckerumwandlungsmöglichkeiten. Abhängig von der jeweiligen Pflanze enthält Nektar relativ hohe Konzentrationen an Saccharose. Laut der aktuellen KEGG-Datenbank ist eine Umwandlung dieses Disaccharides über Glucoinvertase (EC 3.2.1.20) zu Fructose und Glucose möglich. Das entsprechende Gen wurde bei M. plutonius detektiert (Abbildung 10). Eine spezifische Sucrase (Invertase) wurde hingegen in keinem Genom entdeckt. Die von der Larve aufgenommene Zuckerlösung enthält hauptsächlich bereits gespaltene Saccharose, also Fructose und Glucose (Olaitan et al., 2007), welche von P. larvae und $M$. plutonius verstoffwechselt werden können. Interessanterweise ist $P$. larvae in der Lage, extrazelluläre Trehalose über ABC Transporter aufzunehmen und in Glucose zu spalten (Kapitel B2). Trehalose ist ein Hauptbestandteil der Hämolymphe von adulten Honigbienen (Woodring et al., 1993). Geht man davon aus, dass dieses Disaccharid auch in der Hämolymphe von Honigbienenlarven vorkommt, so erscheint die Möglichkeit der Aufnahme und Verwertung des Zuckers als Vorteil. Im Gegensatz zu P. larvae kann der der Larvennahrung zugefügte Pollen von $M$. plutonius für metabolische Zwecke genutzt werden. Es wurden potentielle Gene gefunden, die für den Abbau des Pektinrückgrats von Pollenzellwänden zuständig sind (Kapitel B4). Laut CAZy Datenbank besitzen typische Stämme von $M$. plutonius das genomische Potential, Proteine von 14 verschiedenen Glycosidhydrolase-(GH-) Familien und drei verschiedenen Polysaccharid-Lyase-(PL-) Familien zu bilden, die nach Engel et al. (2012) in den Abbau von pflanzlichen Zellwänden involviert sind. Im Gegensatz dazu besitzen beide P. larvae-Stämme nur das Potential zur Bildung von Proteinen zweier GH-Familien.

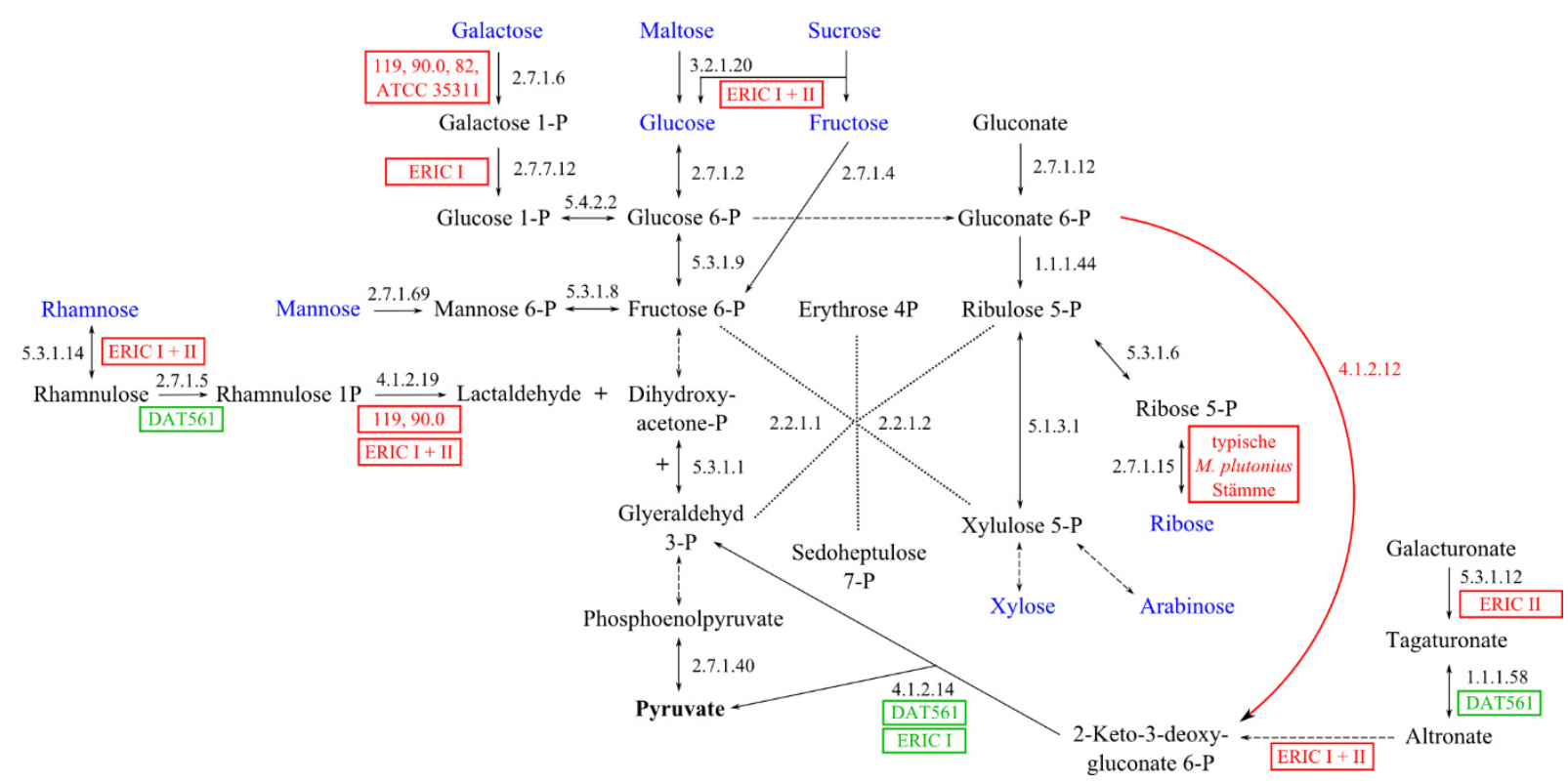

Abbildung 10: Glykolyse, Pentose-Phosphat Weg, Entner-Doudoroff-(ED-) Weg und Zuckerumwandlungen von M. plutonius und P. larvae (editierte Abbildung aus Kapitel B4). 
Die Reaktionen wurden schematisiert. Gepunktete Pfeile indizieren multiple Reaktionsschritte. Genprodukte sind als EC Nummern angegeben. Grüne Blöcke bedeuten, dass nur der angegebene Stamm zu der Reaktion befähigt ist. Stämme in roten Blöcken sind zu der jeweiligen Reaktion nicht befähigt. Zucker, die im Honig und im Pektinrückgrat vorhanden sind, wurden blau markiert. Allen Stämmen fehlt die Phosphogluconat-Dehydrogenase (roter Pfeil, EC 4.1.2.12), welche Teil des ED-Wegs ist. ERIC I = P. larvae DSM 25719, ERIC II = P. larvae DSM 25430.

Sowohl $P$. larvae als auch $M$. plutonius vermehren sich aktiv im Darm der Honigbienenlarve und müssen Substrate für metabolische Zwecke aufnehmen. Beide Organismen sind in der Lage, den durch die Larve aufgenommenen Zucker zu verstoffwechseln. Während $P$. larvae potentielle Nahrungskonkurrenten und Sekundärerreger wahrscheinlich über Bildung von Sekundärmetaboliten eliminiert und sich potentiell auch von deren Überresten ernähren kann, ist M. plutonius wahrscheinlich zur Verwertung von Polleninhalten fähig.

\subsubsection{Tyramin als frühzeitig sekretierter, potentieller Virulenzfaktor}

Biogene Amine (BA) stellen insbesondere in der Lebensmittelindustrie ein Risiko für den Menschen dar (De Palencia et al., 2011; Perin et al., 2014). Bei höheren Organismen führen diese BA zu Hypertonie und Adrenalinfreisetzung (Kanbar et al., 2004). Höhere Konzentrationen zeigen zudem membranolytische Aktivitäten (Kanbar et al., 2004) und somit toxische Effekte. Tyramin gehört $\mathrm{zu}$ den BA und gilt bei verschiedenen Enterokokken und Lactokokken als Virulenz- (Jiménez et al., 2013; Kanbar et al., 2004, 2005; De Palencia et al., 2011) und Fitnessfaktor in Umgebungen mit niedrigem pH-Wert (Perez et al., 2014). Bei M. plutonius wurde ein Gencluster entdeckt, das für die Bildung und Freisetzung von Tyramin verantwortlich ist (Kapitel B4). Dieses Gencluster zeigt sehr hohe DNA-Sequenzähnlichkeiten zu einem Gencluster von E. faecalis. In vitro-Versuche haben gezeigt, dass Tyramin zu einem EFB-typischen Symptom führt: die Gelbfärbung bei infizierten Larven. Außerdem wurde nachgewiesen, dass Tyramin eine schädigende Wirkung auf vier bis fünf Tage alte Honigbienenlarven besitzt (Kanbar et al., 2004, 2005). Die Tyrosin-Decarboxylase ist für die Bildung von Tyramin unter Freisetzung von Kohlendioxid zuständig. Interessanterweise besitzen einige typische M. plutonius Stämme eine Nonsens-Mutation im Gen für die Tyrosin-Decarboxylase. Dies könnte eventuell zu einer verringerten Virulenz führen.

Neue Analysen zeigen, dass auch P. larvae zur Tyraminbildung in der Lage sein könnte.

Das dafür zuständige Protein zeigt eine hohe Sequenzähnlichkeit zu der TyrosinDecarboxylase von M. plutonius. Es wäre also auch möglich, dass die TyrosinDecarboxylase bei P. larvae einen Virulenzfaktor darstellt. Da die entsprechenden Gene 
von ERIC I und II Unterschiede in ihrer DNA-Sequenzlänge aufweisen und sich auch das dazugehörige Gencluster unterscheidet, könnte das zu einer abweichenden Virulenz bei ERIC I und II führen.

Die Wirkung von Tyramin auf Epithelzellen der Honigbiene muss noch untersucht werden. Die Gelbfärbung von EFB-infizierten Honigbienenlarven bereits in einem sehr frühen Stadium der Infektion spricht für eine sehr frühe Bildung des BA. Eine Gelbfärbung bei jungen Honigbienenlarven ist allerdings bei einer AFB-Infektion im Allgemeinen nicht zu sehen. Somit muss zum einen geklärt werden, ob P. larvae Tyramin sekretiert und zum anderen, welcher Prozess zu einer Gelbfärbung bei der EFB-infizierten Larve führt.

\subsection{Invasive Phase der AFB- und EFB-Pathogenese}

Auf die nicht-invasive Phase folgt die invasive Phase, die im Fall einer AFB-Infektion mit dem Tod der Larve einhergeht. Eine EFB-Infektion muss nicht zwangsläufig zum Tod der Honigbienenlarve führen (Abbildung 7). In diesem Abschnitt werden die Unterschiede zwischen der AFB- und EFB-Pathogenese mit den über die Genomanalyse ermittelten Virulenzfaktoren in Verbindung gebracht und zusammengefasst.

\subsubsection{Die Degradation der peritrophischen Matrix (PM) der Honigbienenlarve als Übergang zur invasiven Phase}

Die invasive Phase wird bei der AFB durch die Degradation der PM durch P. larvae eingeleitet (Garcia-Gonzalez \& Genersch, 2013). Die PM kleidet den Darm der Honigbienenlarve aus und besteht aus sekretierten Glycoproteinen (Peritrophine) und Chitin (Terra, 2001). Die PM ist die erste Barriere, die überwunden werden muss, um das Darmepithel zu erreichen und in das Haemocoel vorzudringen (Garcia-Gonzalez et al., 2014c; Yue et al., 2008). P. larvae degradiert die PM durch die Sekretion von PlCBP49, einem essentiellen Virulenzfaktor, der eine Chitinase-ähnliche Funktion besitzt (GarciaGonzalez et al., 2014c). Dieses Enzym stellt zugleich ein neues Mitglied der „Auxiliary Activity 10“ (AA10)-Familie der lytischen Polysaccharid-Monooxygenasen dar (GarciaGonzalez et al., 2014c). Auch M. plutonius ist in der Lage, die PM zu durchbrechen (Alippi, 1999; Shimanuki, 1990). Der genaue Vorgang wurde bislang allerdings nicht geklärt. In silico Analysen haben gezeigt, dass M. plutonius im Genom ein Gen besitzt, welches für ein Protein der AA10-Familie kodiert und Ähnlichkeiten zu PlCBP49 aufweist (Kapitel B4). Auch spricht das Vorhandensein von einem Gene für ein Peptidase-M60Familienprotein (Enhancin) bei M. plutonius für die Fähigkeit, die PM abbauen zu können, 
da diese enzymatische Reaktion bei Enhancinen anderer Organismen bereits belegt wurde (Fang et al., 2009; Tellam et al., 1999; Toprak et al., 2012). Das Genom von P. larvae enthält auch Gene für Peptidasen der M60 Familie, allerdings sind diese Gene möglicherweise durch Nonsens-Mutationen und Transposase-Insertionen defekt. Eine Funktionszuordnung eines M60-Familie-ähnlichen Proteins bei P. larvae DSM 25719 (ERIC I) im AFB-Infektionsverlauf steht noch aus.

Endo-alpha- $N$-acetylgalactosaminidasen katalysieren die Freisetzung von Oligosacchariden über die Hydrolyse der $O$-glycosidischen Bindung zwischen alphaAcetylgalactosamin und Serin-/Threonin-Resten von Proteinen. Ob die Endo-alpha- $N$ acetylgalactosaminidase von einigen $M$. plutonius Stämmen (typische Stämme 21.1, 49.3, 60, B5, H6, L9, S1, 764-5B, 765-6B und der atypische Stamm DAT561) eine Wirkung auf die Glycoproteine der PM hat, ist noch nicht geklärt, wenngleich dieses Enzym bei einigen Bakterien als Virulenzfaktor angesehen werden kann (Kapitel B4) (Ashida et al., 2008; Gregg \& Boraston, 2009).

Der Abbau der PM ist von großer Bedeutung bei der AFB-Pathogenese. P. larvae Stämme, die eine $P l C B P 49-D e l e t i o n(\Delta c b p 49)$ aufweisen, zeigten eine um ca. 95 Prozent verringerte Mortalitätsrate bei AFB-infizierten Honigbienenlarven (Garcia-Gonzalez, et al., 2014c). Eine detaillierte Analyse der potentiell PM-schädigenden Proteine von $M$. plutonius steht noch aus, sollte aber Bestandteil weiterer Analysen sein, da der Abbau der PM auch bei M. plutonius der Startschuss für die invasive Phase darstellen könnte. Denkbare Kandidaten für eine Analyse wären Enhancin und die Endo-alpha- $N$ acetylgalactosaminidase, welche beide während einer EFB-Infektion exprimiert werden. Eine Überprüfung der Expression des PlCBP49-homologen Gens bei M. plutonius wurde noch nicht vorgenommen.

\subsubsection{Durchbruch durch das Darmepithel und die Zersetzung des Wirtskörpers}

Nach dem Abbau der PM ist die Darmepithelzellschicht der Honigbienenlarve ohne Schutz und stellt das nächste Angriffsziel in der AFB- und EFB-Pathogenese dar. Anhand vergleichender Genomanalysen im Jahr 2013 wurden bei P. larvae DSM 25719 (ERIC I) sieben und bei P. larvae DSM 25430 (ERIC II) fünf Toxin-kodierende Genloki festgestellt werden (Kapitel B2). Neuen Analysen zufolge besitzt P. larvae DSM 25719 (ERIC I) allerdings elf und $P$. larvae DSM 25430 (ERIC II) sechs Genloki, die für Toxine kodieren. Dennoch wird bislang vermutet, dass der Genotyp ERIC II aufgrund der Mutationen in den jeweiligen Toxin-bildenden Genen nicht in der Lage ist, Toxine zu produzieren (Kapitel 
B2) (Poppinga \& Genersch, 2015). Dem ERIC I-Stämmen dagegen können bislang zwei Toxine zugeordnet werden, die zu einer Steigerung der Virulenz während einer AFBInfektion führen (Fünfhaus et al., 2013).

Durch die Analyse von 14 M. plutonius-Genomen konnte das erste und bislang einzige Gen ermittelt werden, das für ein potentielles Toxin (Melissotoxin A) kodiert (Kapitel B4). Dieses Toxin-Gen ist im Gegensatz zu den P. larvae-Toxinen nicht chromosomal kodiert sondern liegt plasmidkodiert (pMP19) vor. Die Wirkungsweise des Toxins ist bislang unbekannt. Das entsprechende Gen wird jedoch während der EFB-Pathogenese exprimiert (Kapitel B4). Interessanterweise besitzen nur einige typische Stämme dieses Toxin, nicht aber der atypische Stamm DAT561, obwohl dieser Stamm eine sehr hohe Virulenz aufweist. Die Larven-Mortalitätsrate lag fünf Tage nach einer in vitro Infektion mit $M$. plutonius DAT561 bei 95 Prozent (Arai et al., 2012). Zusätzlich konnte gezeigt werden, dass typische Stämme nur zu einer fünf- bis siebzehnprozentigen Sterberate nach fünf Tagen bei Honigbienenlarven führten (Arai et al., 2012). Die von den Autoren der in vitroInfektionsstudie gewählte sehr kurze Inkubationszeit von insgesamt fünf Tagen ist allerdings kritisch zu betrachten, da Larven auch in einem späteren Stadium, nach dem ersten Abkoten oder als Puppe an einer Infektion sterben können (siehe Entwicklung der Honigbiene in Kapitel A2). Die Überprüfung der Letalität der typischen Stämme wurde somit vernachlässigt. Unabhängig davon ist bekannt, dass atypische Stämme ihre Pathogenität nach einigen Anzuchten verlieren (Arai et al., 2012). Erklärbar wäre dieser Verlust unter anderem durch den Verlust eines über horizontalem Gentransfer vermittelten Elements, in diesem Fall des Plasmids pMP19. M. plutonius verliert nach ungefähr drei bis fünf Anzuchten dieses Plasmid (Kapitel B4). Somit könnte der Verlust des Plasmids und des Toxins bei M. plutonius zu einer verringerten Virulenz oder zu einem Verlust der Pathogenität führen. Weitergehende Analysen sind hier gefordert.

Während die Sekretion von Toxinen beim P. larvae-Genotyp ERIC I eine entscheidende Rolle spielt, ist beim ERIC II-Genotyp ein Oberflächenprotein an der Virulenz beteiligt. Die Fähigkeit von Organismen, an eukaryotischen, extrazellulären Matrixproteinen wie Laminin, Fibronectin, Fibrinogen und Collagen zu binden, wurde schon häufig als virulente Eigenschaft beschrieben (Courtney et al., 1994; Holmes et al., 2001; Massey et al., 2001; Spigaglia et al., 2013). SplA ist ein „S-Layer“-Protein, welches die Bindung von P. larvae DSM 25430 (ERIC II) an Darmepithelzellen der Honigbienenlarve induziert (Poppinga et al., 2012). Ein Verlust dieses Proteins führte zu einer ungefähr 45 Prozent verringerten Larvenmortalität und eine ungefähr um drei Tage verlängerten Larven- 
Überlebensdauer (Poppinga et al., 2012). Ein homologes Protein wurde bei M. plutonius nicht detektiert. Dennoch wurden diverse Gene für Zelloberflächenproteine gefunden, die zur Virulenz des EFB-Erregers über Adhäsion- und Biofilmbildung beitragen könnten (Kapitel B4). Entscheidend dürfte hier auch die Bildung eines „Enterococcal Polysaccharide Antigens“ (Epa) sein, das bei E. faecalis bereits als Virulenzfaktor beschrieben wurde (Rigottier-Gois et al., 2014; Teng et al., 2009). Zudem scheinen typische Stämme in den meisten Fällen nur noch Überreste der Genregionen zu besitzen, die in dem atypischen Stamm für die Oberflächenproteine kodieren. Inwieweit die unterschiedliche Ausstattung an Oberflächenproteinen von typischen und atypischen $M$. plutonius Stämmen zu abweichender Virulenz führen kann, muss in weiterführenden Studien geklärt werden.

P. larvae besitzt eine Reihe von Proteasen, die in M. plutonius nicht vorkommen und gleichzeitig als Virulenzfaktoren bei anderen Organismen beschrieben sind. Die Thermolysin-ähnliche Metalloprotease Bacillolysin (Peptidase der M4 Familie) wird von vier (ERIC I) bzw. drei (ERIC II) verschiedenen, orthologen Genen im Genom von $P$. larvae kodiert und gilt als Virulenzfaktor bei Burkholderia cenocepacia (Kooi et al., 2006), Bacillus pseudomycoides, Bacillus thuringiensis (Luo et al., 2013) und Bacillus anthracis (Chung et al., 2006). Eine Bacillolysin-Protease von B. thuringiensis ist in der Lage, intestinales Gewebe von Caenorhabditis elegans zu zerstören (Luo et al., 2013). Zusammen mit dem Immun-Inhibitor A (nur ERIC II), Collagenasen und einer Hyaluronidase können so unter anderem Zell-Zell-Verbindungen in eukaryotischen Gewebe aufgelöst und für weitere Stoffwechselprozesse zur Verfügung gestellt werden (Kapitel B2) (Chung et al., 2006; Jung et al., 1999; Li et al., 2000; Lindsay et al., 2009). Dem Immun-Inhibitor A werden zudem die Spaltung sekretierter antimikrobieller Peptide des Wirtsorganismus zugeschrieben (Lövgren et al., 1990).

Zusammenfassend lässt sich ableiten, dass P. larvae also mit den genannten Enzymen potentiell in der Lage ist, tiefer in das Gewebe (Haemocoel) der Larve vorzudringen, es abzubauen und dem Immunsystem der Larve zu entkommen.

Die Genomanalysen der vierzehn M. plutonius-Stämme haben gezeigt, dass das genetische Repertoire zur Überwindung von Zell-Zell-Barrieren sehr begrenzt ist. Bislang konnte nur eine putative Collagenase der U32 Peptidasefamilie identifiziert werden (Kapitel B4). EFB und AFB zeigen im späteren Verlauf der Krankheit unterschiedliche Symptome (Kapitel A3.1). Während P. larvae die tote Larve vollständig zu einer braunen, zähflüssigen Masse zersetzt, trocknet die Larve bei einer EFB-Infektion zu einem festen Schorf ein. Die These, 
dass der Primärerreger der EFB nicht in der Lage ist, sämtliches Bienenlarvengewebe zu zersetzen, wird somit durch die Genomanalysen der vierzehn Stämme untermauert. Der bereits erwähnte Einfluss von möglichen Sekundärerregern, potentiellen Saprophyten (Forsgren, 2010), könnte zum Zersetzungsprozess der Honigbienenlarve beitragen.

Zusammenfassend ist $\mathrm{zu}$ erkennen, dass sich die genetische Ausstattung an Virulenzfaktoren bei $P$. larvae und $M$. plutonius unterscheidet. Ferner besitzen die $P$. larvae-Genotypen (Kapitel B2) als auch teilweise die einzelnen M. plutonius-Stämme (Kapitel B4) eine verschiedenartige genetische Ausstattung. Das lässt darauf schließen, dass sich auch innerhalb der jeweiligen Art unterschiedliche Pathogenitätsmechanismen entwickelt haben und nicht nur Unterschiede zwischen $P$. larvae und $M$. plutonius bestehen.

\subsection{Genomreduktion als Zeichen der Evolution: Der Einfluss von Phagen, mobilen genetischen Elementen und Nonsens-Mutationen auf die Virulenz der Erreger}

Bislang wurden noch keine Studien zur Evolution der Erreger P. larvae und M. plutonius durchgeführt. Man kann allerdings davon ausgehen, dass die bei beiden Erregern auftretende Genomreduktion über Transposasen, Phagen und Mutationen ein evolutiver Prozess ist und eine Anpassung an die Lebensumstände darstellt (McCutcheon \& Moran, 2011). Die Genomreduktion stellt wahrscheinlich eine Anpassung der Bakterien an den Wirt, die Honigbienenlarve, und damit verbunden an ein Leben in einem Honigbienenvolk dar. Man geht von der Hypothese aus, dass sich endosymbiotisch lebende Bakterien aus freilebenden wirtsunabhängigen Bakterien im Laufe der Evolution entwickelt haben (McCutcheon \& Moran, 2011; Nilsson et al., 2005), und dass die evolutive Anpassung an den Wirt durch die Genomreduktion angetrieben wird (Wolf \& Koonin, 2013). Ein allgemeines Modell für diesen Prozess ist in Abbildung 11 aufgezeigt. 


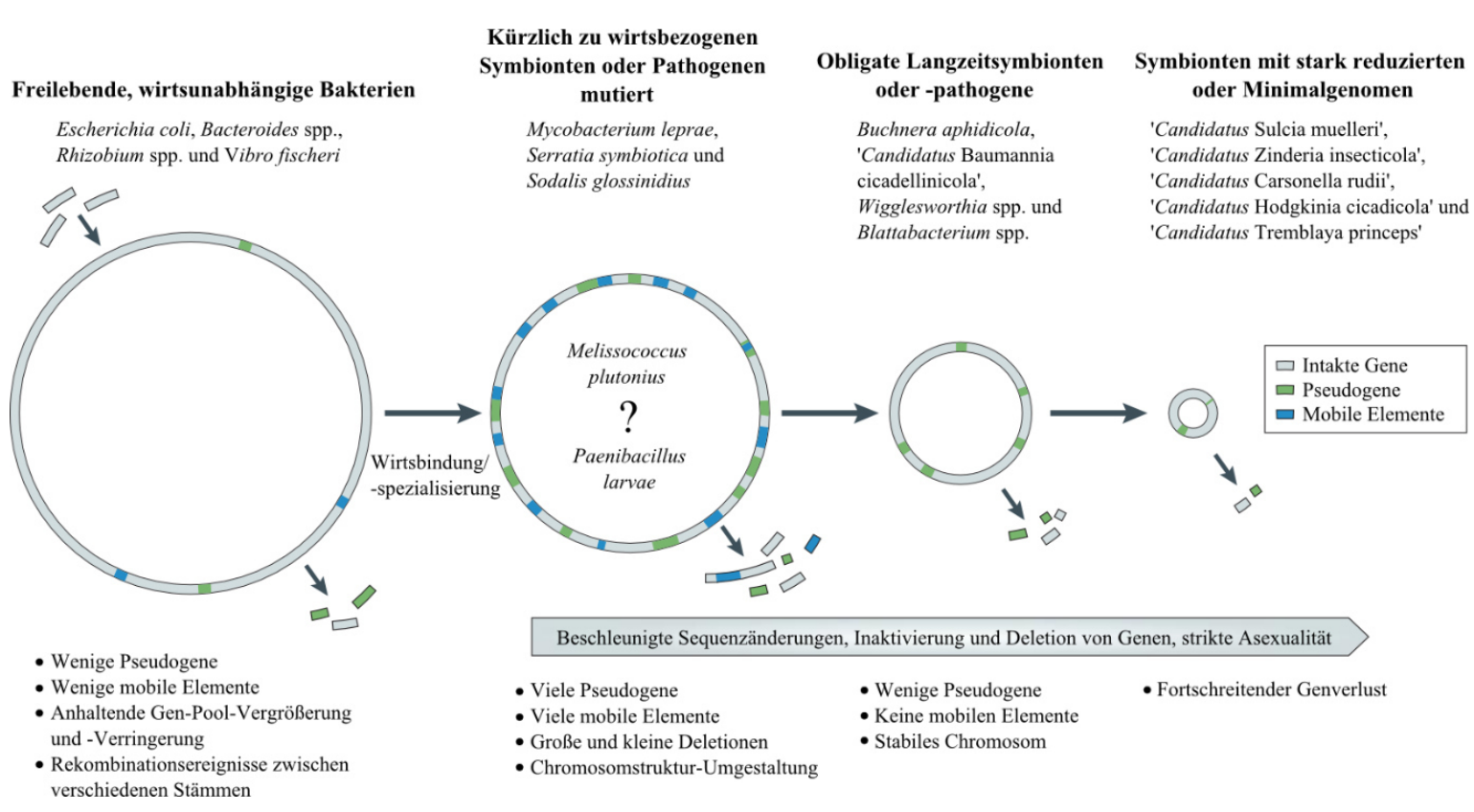

Abbildung 11: Stadien der Genomreduktion in wirtsabhängigen Bakterien. Die Abbildung entstammt aus McCutcheon \& Moran (2011) und wurde übersetzt. Das mutmaßliche Stadium von $P$. larvae und M. plutonius wurde hinzugefügt.

Im Rahmen der Genomanalysen der bakteriellen Erreger P. larvae und M. plutonius konnten Phagen, Pseudogene und mobile genetische Elemente identifiziert werden, die einen Einfluss auf die Virulenz der Organismen haben. Es ist bereits bekannt, dass sowohl Virulenz- als auch Fitnessfaktoren über mobile genetische Elemente wie Transposons, Plasmide, Bacteriophagen oder Pathogenitätsinseln kodiert und über horizontalem Gentransfer übertragen werden können (Frost et al., 2005). Als Beispiele hierfür gelten das Shiga-Toxin von enterohaemorragischen Escherichia coli (EHEC)-Stämmen oder das Botulinum-Toxin von Clostridium botulinum, die Phagen-assoziiert sein können (Adams et al., 2014; Brüssow et al., 2004). In den Genomen von P. larvae ERIC I und ERIC II wurden 22 bzw. 8 Phagenregionen ermittelt. Dagegen konnten in den M. plutonius Genomen nur ein (atypischer Stamm) bis drei Phagenregionen gefunden werden. Während bei M. plutonius Bacteriocin-kodierende Gene innerhalb dieser Regionen gefunden wurden (Kapitel B4), trägt der von Oliveira et al. (2013) ermittelte Phage phiIBB_Pl23 ein Plx1Toxin-Homolog, welches während der AFB Pathogenese eine Rolle spielt (Fünfhaus et al., 2013). Zwei nahezu identische Kopien dieses Gens liegen im Genom von ERIC I vor (Abbildung 12). 


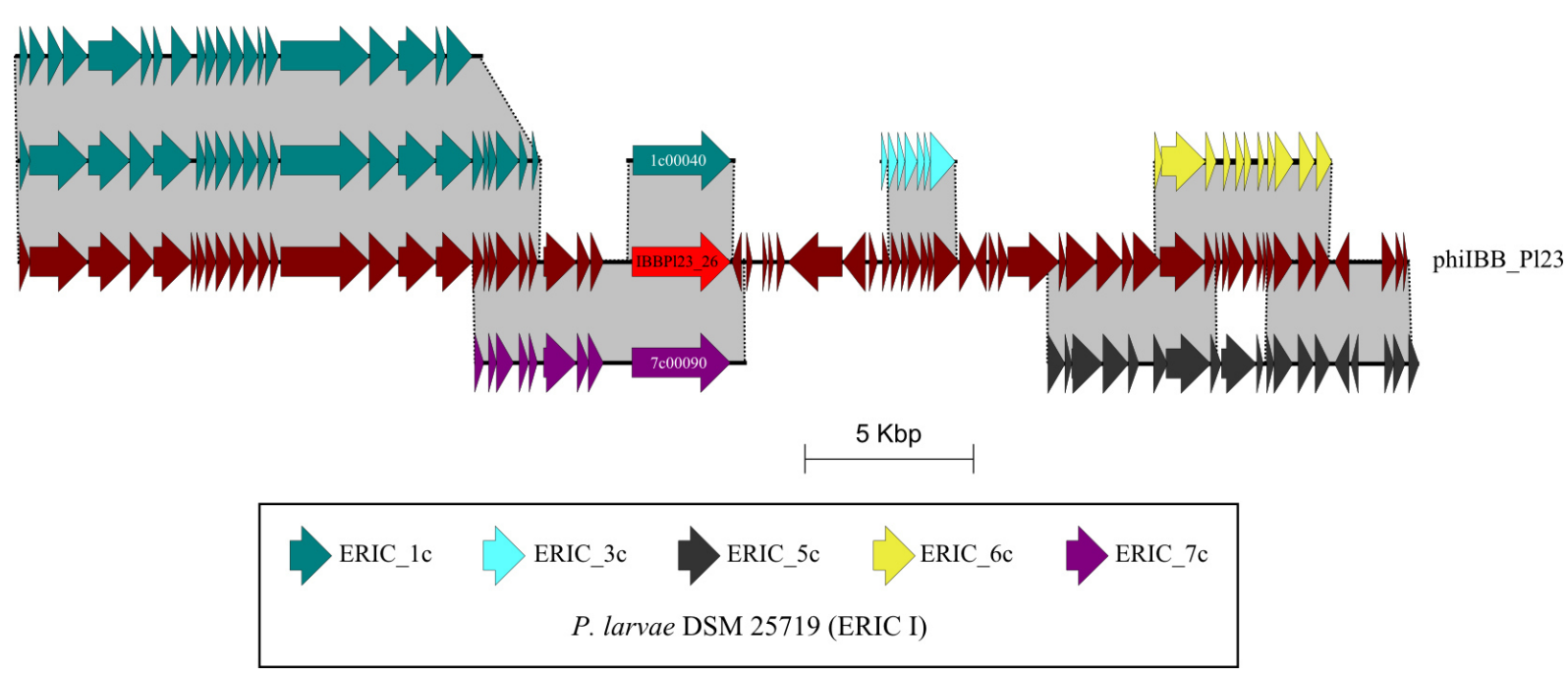

Abbildung 12: Vergleich von Phage phiIBB_Pl23 mit dem Genom von P. larvae DSM 25719 (ERIC I).

Abgebildet sind die Bereiche des Genoms von P. larvae DSM 25719 (ERIC I), die hohe DNASequenzähnlichkeiten zu dem Phagen phiIBB_Pl23 aufweisen. Das Toxin-kodierende Gen von philBB_Pl23 ist als roter Pfeil gekennzeichnet. Homologe Bereiche sind in grau markiert. Bis auf das Toxin-kodierende Gen besitzen alle abgebildeten ORFs hohe Ähnlichkeiten zu Phagenkodierenden Genen. Jedes Contig besitzt eine eigene Farbe.

Pseudogene fungieren als Relikte von Proteinen oder Stoffwechselwegen, die das Bakterium aufgrund seiner Anpassung an die neue Umgebung nicht mehr benötigt (Goodhead \& Darby, 2015). Es wurden bei P. larvae DSM 25719 (ERIC I) 75 Pseudogene und bei DSM 25430 (ERIC II) 99 potentielle Pseudogene nachgewiesen (Kapitel B2). Dies entspricht 1,5 bzw. 2,5 Prozent der proteinkodierenden Sequenzbereiche der Genome. In den Genomen von M. plutonius wurden Pseudogene in höherer Abundanz ermittelt: Zwischen 75 bis 156 Pseudogene wurden gefunden, die wiederum 4,5 bis 8,9 Prozent der proteinkodierenden Sequenzbereiche ausmachen. Auch hier sind in beiden Organismen Fitness- und Virulenzfaktoren betroffen. Mutationen in den Genen von einigen Collagenasen, Chitinasen und dem Immun-Inhibitor A haben bei ERIC I zu Pseudogenen geführt. Auch bei M. plutonius sind Virulenzfaktoren wie das Tyrosin-Decarboxylase-Gen oder Oberflächenprotein-Gene betroffen.

Transposasen als Teil mobiler, genetischer Elemente katalysieren die intrazelluläre Umgestaltung des Genoms (Frost et al., 2005). Im Gegensatz zu den 256 (ERIC I) bzw. 366 (ERIC II) Transposasen, die von P. larvae kodiert werden, wurden bei M. plutonius nur drei Integrasen identifiziert. Die Transposasen bei P. larvae DSM 25719 und DSM 25430 besitzen wie die Prophagenbereiche und Nonsens-Mutationen einen Einfluss auf die Virulenz des Erregers. So sind durch Transposase-Insertionen Virulenzfaktoren wie 
Enhancin, Bacteriocin-Synthese oder auch Toxin-kodierende Gene betroffen und wahrscheinlich nicht mehr funktionell (Kapitel B2).

Die Genome freilebender Bakterien besitzen einen höheren GC-Nukleotid-Gehalt als die von spezialisierten, wirtsbezogenen Bakterien (McCutcheon \& Moran, 2011; Moran, 2002; Rocha \& Danchin, 2002). Zudem wird eine verringerte Fähigkeit zur Bildung essentieller Aminosäuren als eine weitere Anpassung und Bindung an den Wirtsorganismus angesehen (Bennett et al., 2014). Aufgrund der geringen Genomgröße von M. plutonius und dem niedrigen GC Gehalt des Genoms (Abbildung 13), der hohen Anzahl an Pseudogenen, dem eingeschränkten Wachstum unter sauerstoffhaltiger Atmosphäre und der Auxotrophie gegenüber 15 bis 18 Aminosäuren, kann davon ausgegangen werden, dass sich dieser Organismus in einen fortgeschrittenen Zustand der Anpassung an die Honigbienenlarve befindet. P. larvae besitzt im Vergleich zu anderen Paenibacillen ein reduziertes Genom und auch einen vergleichsweise niedrigen GC-Gehalt (Abbildung 13). Wie bereits beschrieben, ist die Anzahl an mobilen Elementen hoch. Deswegen kann man auch hier von einer Anpassung des Genoms an das Habitat „Honigbienenlarve“ ausgehen.

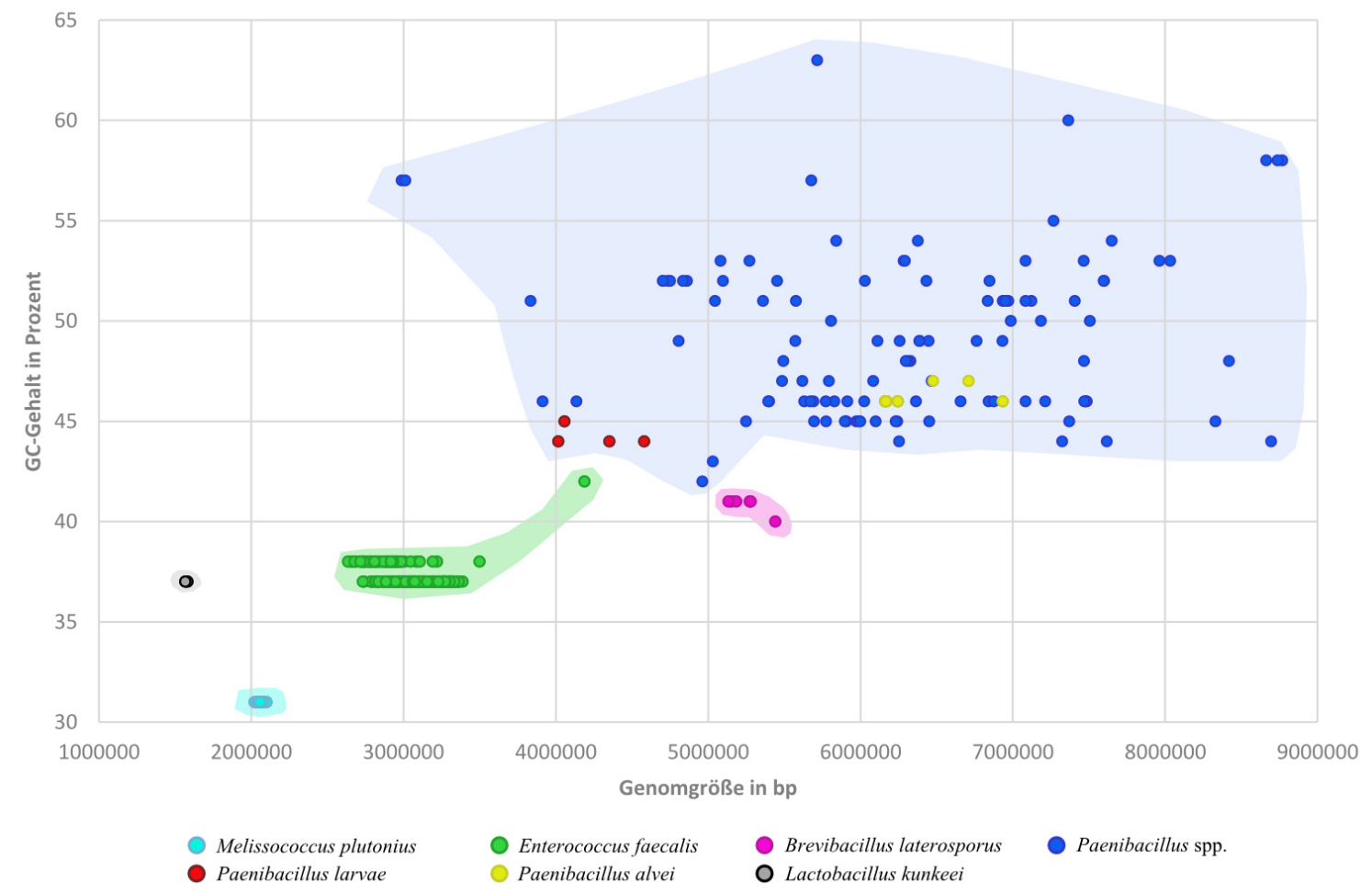

Abbildung 13: Übersicht über die Genomgrößen in Relation zum GC-Gehalt von AFB- und EFBassoziierten Bakterien. Für die Abbildung wurden alle Genome von Melissococcus plutonius (14), Paenibacillus larvae (4), Enterococcus faecalis (370), Paenibacillus alvei (10), Brevibacillus laterosporus (6), Lactobacillus kunkeei (2) und Paenibacillus spp. (108) aus der IMG/ER 
Datenbank (Markowitz et al., 2009) verwendet. Die Paenibacillus spp. (P. larvae und P. alvei ausgenommen) wurden zu Vergleichszwecken hinzugefügt.

\section{Initiale Genomanalyse von Sekundärerregern der Europäischen Faulbrut}

Br. laterosporus, P. alvei, E. faecalis und A. eurydice sind laut Literatur häufig auftretende Sekundärerreger der EFB (Forsgren, 2010). Die Bedeutung dieser Organismen während einer EFB Infektion ist nicht geklärt, obwohl Br. laterosporus und $P$. alvei die Rolle der Saprophyten zugesprochen wird (Bailey, 1963b; Forsgren, 2010). Gencluster aus M. plutonius, die für Virulenzfaktoren kodieren, zeigen oftmals sehr hohe Ähnlichkeiten zu Genclustern oder Genen aus E. faecalis (Kapitel B4). M. plutonius steht in einer nahen phylogenetischen Verwandtschaft zum Genus Enterococcus (Cai \& Collins, 1994). Im Jahr 1963 hat Bailey Koinfektionsstudien durchgeführt und kam zu dem Ergebnis, dass $M$. plutonius zwar der Haupterreger der EFB ist, beide Bakterien zusammen aber eine verstärkende Wirkung auf die Virulenz besitzen (Bailey, 1963b).

Im Rahmen dieser Arbeit konnten $P$. alvei Stämme aus verschiedenen EFB-infizierten Honigbienenlarven isoliert werden, wenngleich Phäno- und Genotypisierung noch ausstehen.

In den Genomen von P. alvei DSM 29 und Br. laterosporus LMG 15441 wurden eine Reihe von Virulenzfaktoren ermittelt, die potentiell Einfluss auf den Verlauf der EFBPathogenese haben könnten (Kapitel B5 und B6). Die hohe Anzahl an PKS-, NRPS- und NRPS/PKS Hybrid-Genregionen in beiden Organismen ist auffällig. Br. laterosporus besitzt 13 Gencluster zur Biosynthese von Sekundärmetaboliten (Weber et al., 2015), ein Siderophor-assoziiertes Gencluster und zwei Regionen, die für potentielle Bakteriocine kodieren. In früheren Studien wurde gezeigt, dass Br. laterosporus zur Bildung von nichtribosomal hergestellten Peptiden in der Lage ist, die den Tupuseleiamiden (Barsby et al., 2002), Basilikamiden (Barsby et al., 2002), Loloatinen (Gerard et al., 1996, 1999), Tauramiden (Desjardine et al., 2007) und Bogorolen (Barsby et al., 2001, 2006) zugeordnet werden können und antimykotische und antibakterielle Eigenschaften besitzen. Im Genom von P. alvei DSM 29 sind mindestens 14 NRPS- bzw. NPRS/PKS Hybridgencluster kodiert. Zudem besitzen beide Stämme Gene zur Synthese von Bacteriocinen (Zhao et al., 2012). Die Sekretion solcher Sekundärmetabolite und Bacteriocine hat wahrscheinlich einen Einfluss auf die Zusammensetzung der im Darm der Honigbienenlarve vorkommenden Bakteriengemeinschaft. Sowohl Br. laterosporus LMG 15441 als auch $P$. alvei DSM 29 besitzen das Potential zur Bildung von Chitinasen, die am Abbau des Larvenkadavers oder der PM der Honigbienenlarve beteiligt sein könnten. 
Damit hätten beide Stämme wahrscheinlich auch die Möglichkeit, den Start der invasiven Phase von M. plutonius zu beeinflussen. Die antimykotische und insektizide Wirkung einer Chitinase von Br. laterosporus wurde von Prasanna et al. (2013) nachgewiesen. Ferner wurde dieses Bakterium als Invertebratenpathogen beschrieben (Logan et al., 2002; Pessanha et al., 2015; Ruiu, 2013; Ruiu et al., 2012). Das Vorhandensein von jeweils vier verschiedenen Toxin-kodierenden Genen bei $\mathrm{Br}$. laterosporus und $P$. alvei könnte für einen Beitrag während der invasiven Phase der EFB Pathogenese sprechen. Das von $B r$. laterosporus LMG 15441 kodierte epsilon Toxin (BRLA_c21160) weist signifikante Protein-Sequenzähnlichkeiten zu dem von M. plutonius kodierten Melissotoxin A auf. Ein epsilon Toxin stellt ein potentiell porenbildendes, zellmembranschädigendes Protein dar (Petit et al., 2001). Ein weiteres Toxin von Br. laterosporus LMG 15441 besitzt hohe Protein-Sequenzähnlichkeit zu dem P. alvei-spezifischen Toxin „Alveolysin“. Dieses stellt ein Mitglied der sogenannten „Thiol-aktivierten Zytolysine“ (TACY) dar, denen eine Immunabwehr-bekämpfende Rolle während einer Pathogenese zugesprochen wird (Billington et al., 2000; Geoffroy et al., 1990). Zwei weitere, Toxin-kodierende Gencluster von $P$. alvei DSM 29 sind in Abbildung 14 ersichtlich. Eines dieser Toxine ist ein ToxinKomplex (Tc), welcher aus drei Untereinheiten besteht und von diversen Organismen kodiert wird. Die Funktionsweise dieses Komplexes wurde erst kürzlich von Meusch et al. (2014) aufgeklärt (Abbildung 14A). Dabei wird ein von zwei Untereinheiten umgebenes Toxin über eine dritte, membranperforierende Komponente in eukaryotische Zellen geschleust und verursacht dort den Zelltod (Simon et al., 2014). Ein weiteres Toxin von $P$. alvei DSM 29 (Abbildung 14B) zeigt hohe DNA-Sequenzähnlichkeit zu einem Bereich der Tx6-Toxin-Genregion aus P. larvae DSM 25719 (ERIC I). Die Wirkungsweise ist bislang unklar. Auch zeigen zwei binäre Toxine von $B r$. laterosporus LMG 15441 Proteinsequenzähnlichkeiten zu den Toxinen Plx2, Plx3 und Plx4 von P. larvae (Kapitel B2). Sowohl P. alvei als auch Br. laterosporus sind somit potentiell zur Bildung von Toxinen befähigt. Die Wirkung dieser Toxine auf die Epithelzellen von Larven sollte Ziel weiterer Forschungstätigkeiten sein. 


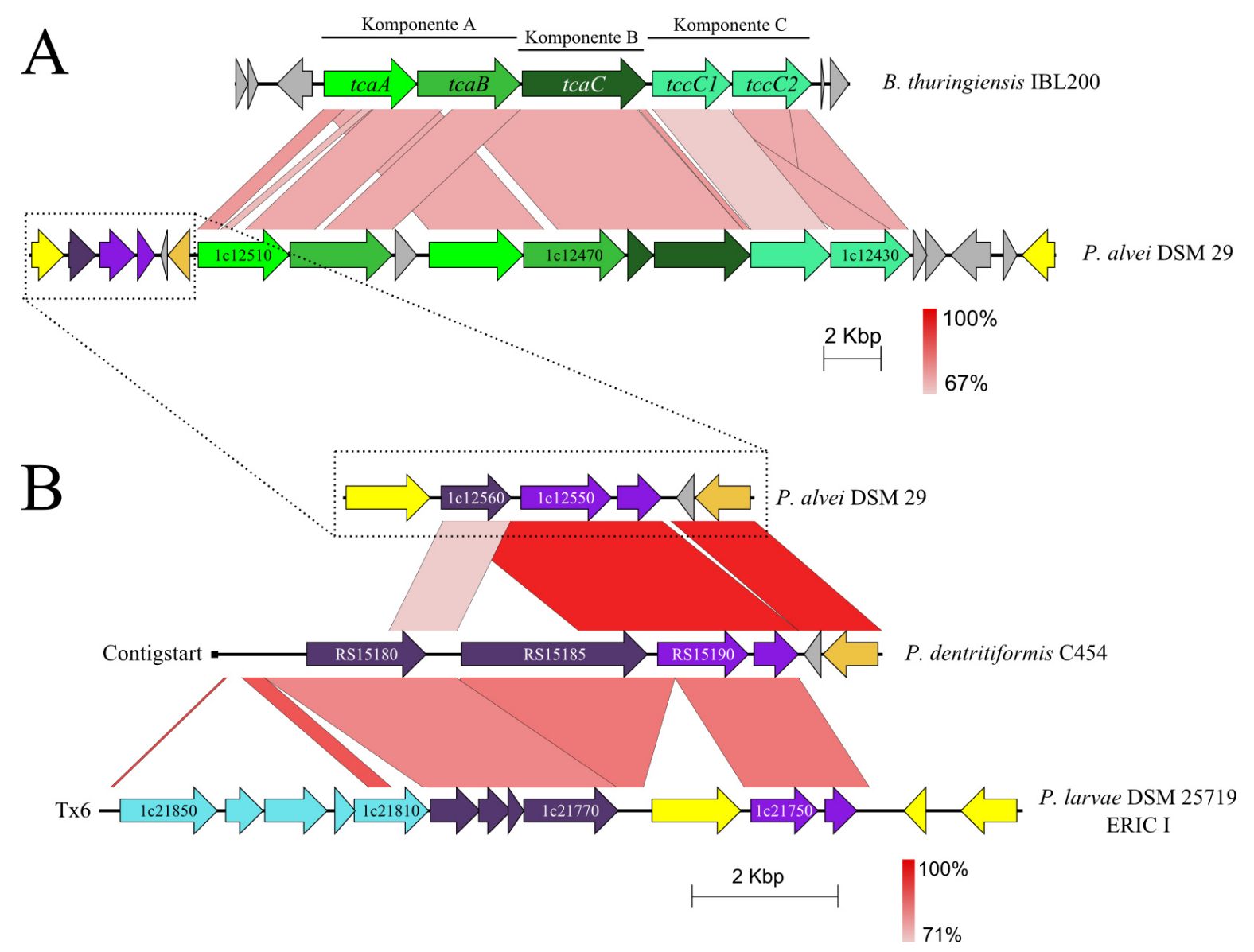

Abbildung 14: Vergleich von Toxin-kodierenden Genregionen im Genom von P. alvei DSM29. Das Tc-kodierende Gencluster (A) ist in verschiedenen Grüntönen markiert und zeigt hohe DNASequenzähnlichkeit zu dem $t c$-Lokus von B. thuringiensis IBL200 (Blackburn et al., 2011). Ein solcher Tc besteht aus drei Komponenten (A, B und C). Flankiert ist der tc-Lokus von verschiedenen Transposasen (gelb) und einem Phagen-assoziierten Gen (ocker). Außerdem befindet sich eine Toxin-kodierende Genregion in direkter Nachbarschaft, die in verschiedenen Lila-Tönen markiert und in B vergrößert abgebildet wurde. Die Toxin-kodierende Genregion (B) von $P$. alvei DSM 29 besitzt wiederum hohe DNA-Sequenzähnlichkeit zu einer Region im Genom von $P$. dentritiformis $\mathrm{C} 454$ und der Tx6-Region (cyan und lila) von P. larvae DSM 25719 (Genotyp ERIC I) (Kapitel B2). Grau markiert sind genomischer Kontext.

Nach der Zerstörung der Epithelzellen in der invasiven Phase der AFB Pathogenese folgt das Vordringen von $P$. larvae in das Haemocoel und die vollständige Degradation des Larvenkadavers (Kapitel B2). Br. laterosporus LMG 15441 weist die genetische Ausstattung zur Bildung von Collagenasen, Bacillolysin (Tian et al., 2007) und ImmunInhibitor A auf, P. alvei besitzt dagegen Collagenase- und Hyaluronidase-kodierende Gene. Vorausgesetzt beide Sekundärerreger sind in der Lage die PM abzubauen, Epithelzellen anzugreifen und extrazelluläre Matrixproteine zu degradieren, kann das auch zu einem Vordringen des Primärerregers M. plutonius in das Haemocoel bedeuten, ohne dass dieser die dafür notwendigen Enzyme besitzen muss. Aktuelle Analysen haben allerdings gezeigt, dass die Sekundärerreger nicht in jeder EFB-infizierten Larve 
vorkommen. Auch ist bekannt, dass die Sekundärerreger nicht für die EFB-Infektion verantwortlich sind, sondern diese allein von M. plutonius ausgelöst wird (Bailey, 1983). Jedoch ist anzunehmen, dass die hier analysierten Sekundärerreger nicht nur die Rolle der Saprophyten einnehmen können, sondern potentiell auch die EFB-Pathogenese beschleunigen und die Larvenmortalität erhöhen könnten.

\section{Die Rolle von Lactobacillus kunkeei als potentieller EFB-Antagonist und}

\section{Fructobacillus sp. EFB-N1 als fructophiles Bakterium}

Die mikrobielle Gemeinschaft im Darm der Honigbienenlarve unterscheidet sich je nach larvalem Stadium (Vojvodic et al., 2013), nach Standort (Hroncova et al., 2015) und wahrscheinlich auch nach Jahreszeit. Zudem kotet die Larve am zehnten Tag ihrer Entwicklung ab und geht in das Puppenstadium über, bei der keine externe Nahrung aufgenommen wird. Die schlüpfende, adulte Biene hingegen wird von Arbeiterinnen gefüttert und entwickelt eine Darmbakteriengemeinschaft, die sich von der der Larve unterscheidet (Ahn et al., 2012) und von acht bakteriellen Phylotypen dominiert wird, die fünf verschiedenen Bakterienklassen angehören (Abbildung 15).

Phylogenie der bakteriellen Phyla

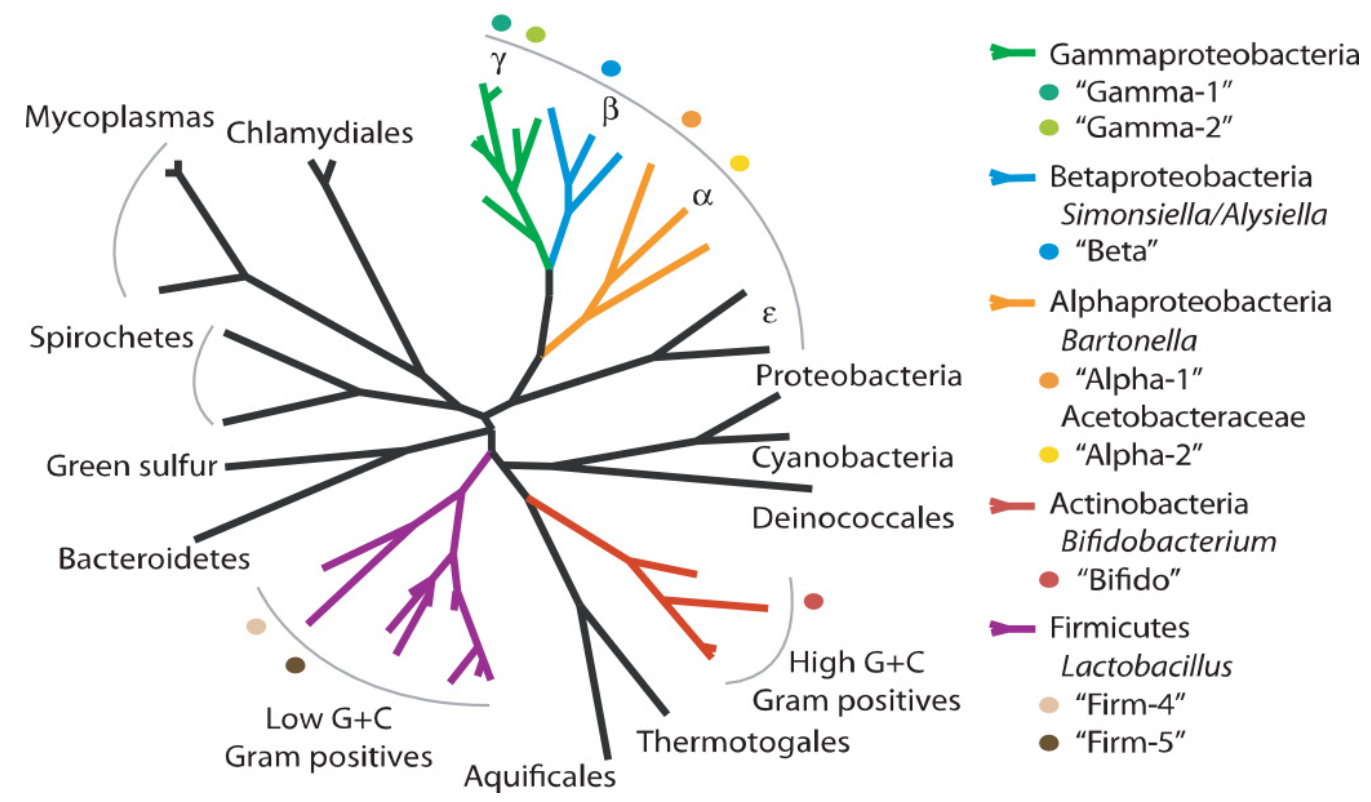

Abbildung 15: Phylogenetische Positionen der bakteriellen Gemeinschaft des Honigbienendarms. Die Abbildung entstammt aus Martinson et al. (2011).

Faktoren wie Honig, Gelee Royale, Nektar, Pollen bzw. Bienenbrot und Sekrete der Ammenbienen spielen eine Rolle für die Zusammensetzung und Diversität des Darmmikrobioms der Larve. L. kunkeei wurde bereits in diversen Habitaten nachgewiesen, 
darunter im Wein (Edwards et al., 1998), an Blumen (Neveling et al., 2012), Pollen und im Honig (Endo \& Salminen, 2013). Eine besondere Nische besetzt das Bakterium im Honigmagen von adulten Bienen (Olofsson \& Vásquez, 2008; Vásquez et al., 2012). Wie Vertreter der Gattung Fructobacillus gehört auch L. kunkeei zu den fructophilen Bakterien, d.h. das Fructose der Glucose als Kohlenstoffquelle vorgezogen wird (Endo \& Salminen, 2013; Endo et al., 2011; Neveling et al., 2012). Es war auch bekannt, dass L. kunkeei in einer antagonistischen Beziehung zu M. plutonius steht (Vásquez et al., 2012). Neue Studien zeigen, dass L. kunkeei häufig in einer hohen relativen Abundanz in EFBinfizierten Larven vorkommt aber auch Bestandteil der gesunden Honigbienenlarvengemeinschaft sein kann (Moran, 2015). Der im Rahmen dieser Arbeit analysierte Stamm EFB6 wurde aus einer EFB-infizierten Larve aus einem Deutschen EFB Ausbruch isoliert. Die Foki der Genomanalyse lagen zum einen auf der Ermittlung von Zelloberflächenproteinen, die eine Biofilmbildung oder eine Bindung an eukaryotische Zellen im Honigbienenmagen ermöglichen, und zum anderen auf antibakterielle Peptide, die L. kunkeei zum Verteidigen seiner Nische nutzen könnte. Vásquez et al. (2012) erzielte mit einer Kombination aus verschiedenen Milchsäurebakterien und L. kunkeei die besten Ergebnisse, die Larvenmortalität bei einer EFB-Infektion zu verringern, wenngleich die Reduktion nur ungefähr 20 Prozent betrug. Butler et al. (2013) vermutet, dass Milchsäurebakterien über die Bildung von Bacteriocinen, Milchsäure, Ameisensäure, Essigsäure und Wasserstoffperoxid die Ausbreitung von M. plutonius im Darm der Larve verhindern oder zumindest verlangsamen können. Im Genom von Stamm EFB6 sind Gene für die Synthese von Bacteriocinen kodiert, die möglicherweise $M$. plutonius entgegenwirken könnten. Eine Analyse dieser Bacteriocine steht allerdings noch aus. Auch wurde bislang nicht bewiesen, dass L. kunkeei alleine M. plutonius inhibieren kann.

Wie bereits erwähnt, ist die hohe relative Abundanz von L. kunkeei in EFB-infizierten Larven beachtenswert. Im Laufe der Jahrzehnte isolierten Forscher neben dem Haupterreger am häufigsten ein Bakterium mit dem Namen Bacterium eurydice (später Achromobacter eurydice) aus EFB-infizierten Larven (Bailey \& Gibbs, 1962; Bailey, $1957 a, 1957 b, 1963 a)$, das eindeutig von E. faecalis, P. alvei und Br. laterosporus abgegrenzt werden konnte. In den Zwanzigern bis Vierzigern des letzten Jahrhunderts wurde sogar postuliert, dass A. eurydice eine „Variante“ von M. plutonius wäre und sich im Darm der Larve zu dem Erreger ,umwandeln würde“. Interessanterweise konnten in aktuellen Studien bislang alle Sekundärerreger bis auf A. eurydice nachgewiesen werden. Neue Analysen basieren allerdings auf der Untersuchung der 16S rRNA-Gensequenzen. 
Die 16S rRNA Gensequenz von A. eurydice wurde bislang nicht ermittelt. Auch steht dieser Organismus in keiner nationalen oder internationalen Kulturensammlung zur Verfügung. Shrivastava vermutete 1982, dass A. eurydice aufgrund von phänotypischen und biochemischen Studien dem Genus Lactobacillus zuzuordnen wäre (Shrivastava, 1982). Zudem führt der von Bailey beschriebene Agar zur Isolierung von A. eurydice (Bailey, 1963a) unter anaeroben Bedingungen aus infizierten Larven, Körperregionen der adulten Honigbiene, Pollen oder Blumen, zur Isolierung von L. kunkeei (Shrivastava, 1982, und eigene Analysen), nicht aber zu Spezies der Gattung Achromobacter. Wie bereits beschrieben, wird L. kunkeei auch mit genau diesen Habitaten in Verbindung gebracht. Zusammenfassend liegt somit die Vermutung nahe, dass es sich bei A. eurydice um $L$. kunkeei handelt.

Im Gegensatz zu Lactobacillus stellt Fructobacillus keine dominante Gattung im Darm der Honigbienenlarve dar und wurde bislang in diesem Zusammenhang nicht näher erforscht. Im Rahmen dieser Arbeit wurde das Bakterium Fructobacillus sp. EFB-N1 aus einer EFBinfizierten Honigbienenlarve isoliert. Durch die initiale Genomanalyse von Fructobacillus sp. EFB-N1 konnte gezeigt werden, dass das Bakterium nah verwandt mit der Spezies $F$. tropaeoli ist. Fructobacillen wurden bislang in keinem EFB-Ausbruch detektiert. Sowohl Vertreter der Gattung Fructobacillus als auch Lactobacillus spp. besiedeln HonigbienenBrutzellen, Bienenbrot und Nektar. Nektar enthält je nach Pflanze relativ hohe Konzentrationen an Saccharose. Sowohl L. kunkeei als auch Fructobacillus sp. EFB-N1 sind über Invertase oder Sucrase-Isomaltase in der Lage, Saccharose in Fructose und Glucose zu spalten. Es wird vermutet, dass Fructobacillen durch ihre Stoffwechseltätigkeiten das Wachstum essentiell notwendiger Bakterien im Darm der Honigbienenlarve animieren (Rokop et al., 2015). Die Bereitstellung von Monosacchariden wie Glucose und Fructose könnte diese These unterstützen. Die Bedeutung der Fructobacillen muss in weiterführenden Analysen untersucht werden.

\section{Ausblick}

Im Rahmen dieser Arbeit konnten über in silico-Analysen potentielle Virulenzfaktoren von den Primärerregern der $\mathrm{AFB}(P$. larvae) und $\mathrm{EFB}(M$. plutonius $)$, und von Sekundärerregern der EFB, Br. laterosporus und $P$. alvei, ermittelt werden. Mithilfe weiterführender Experimente sollte es möglich sein, die Wirkungsweise einzelner Virulenzfaktoren sowohl in vitro als auch in vivo näher zu untersuchen. 
Ein erster Ansatzpunkt stellt das in dieser Arbeit identifizierte und analysierte Virulenzplasmid pMP19 von M. plutonius dar, welches Gene für ein Matrixbindeprotein und ein Melissotoxin trägt. Die Vermutung liegt nahe, dass pMP19-tragende Stämme virulenter sind als Stämme ohne dieses Plasmid. Ein erster Schritt zur Ermittlung der Bedeutung dieses Plasmids wären Infektionsversuche von pMP19-tragenden M. plutoniusStämmen mit Honigbienenlarven. Da M. plutonius das Virulenzplasmid nach einigen Kultivierungsschritten verliert, sollte man parallel dazu Infektionsversuche mit denselben Stämmen durchführen, die das Plasmid verloren haben. Über die Ergebnisse könnte gezeigt werden, ob das Plasmid und die darauf kodierten Gene eine Wirkung während der EFB-Pathogenese besitzen. Wenn mit diesem Versuch bestätigt werden kann, dass das Vorhandensein des pMP19-Plasmids zu einer erhöhten Virulenz führt, wäre eine Analyse der einzelnen Gene des Plasmids sinnvoll. Die Bedeutung chromosomal kodierter Virulenzfaktoren, wie beispielsweise die Tyrosin-Decarboxylase oder das PlCBP49homologes Protein, könnten über knock-out-Studien in Verbindung mit Infektionsstudien ermittelt werden.

Ferner wäre es wichtig, die Bedeutung der Sekundärerreger während der EFB-Pathogenese näher zu erforschen. Dazu gehören zum einen Infektionsassays mit den Sekundärerregern $B r$ laterosporus, $P$. alvei und E. faecalis. Ko-Infektionsstudien, in denen eine Kombination von $M$. plutonius mit jeweils einem oder mehreren Sekundärerregern auf erhöhte Larvenmortalität getestet wird, könnten Auskunft über den Einfluss dieser Organismen auf die EFB-Pathogenese geben. In dieser Arbeit wurden die Genome von $P$. alvei und Br. laterosporus initial analysiert. Weitergehende in silico-Analysen sind notwendig, um eine vollständige Übersicht über alle Virulenzfaktoren zu erlangen. Auch aus EFB-infizierten Honigbienenlarven isolierte E. faecalis-Stämme sollten einer Genomanalyse unterzogen werden. Es wäre interessant $\mathrm{zu}$ erforschen, inwieweit sich solche Stämme von anderen E. faecalis-Stämmen unterscheiden. Nachfolgend könnte man die einzelnen Virulenzfaktoren der Sekundärerreger auf Wirksamkeit gegen Honigbienenlarven testen.

Im Gegensatz zu M. plutonius wurde die Wirkungsweise verschiedener Virulenzfaktoren bei $P$. larvae bereits beschrieben. Die Erforschung bisher wenig oder gar nicht untersuchter Toxine bei P. larvae ERIC I über etablierte knock-out- und Infektionsstudien sollten der Aufklärung der AFB-Pathogenese dienen. Mehr Fragen sind bei der ERIC II-spezifischen AFB-Pathogenese offen. Die bei ERIC II ermittelten Toxin-kodierenden Gene und Genregionen werden bislang als funktionsunfähig angesehenen, da sie nur Bruchstücke der 
in ERIC I vorkommenden, Toxin-kodierenden Regionen darstellen. Nicht immer sind allerdings alle Gene eines Toxin-kodierenden Genclusters notwendig, um ein funktionales Toxin zu exprimieren. Diesen Regionen sollte daher dennoch Beachtung geschenkt werden. Auch hier könnten knock-out- und Infektionsstudien zu einem besseren Verständnis der AFB-Pathogenese beitragen.

Pathogenitätsmechanismen wie die Spaltung von antimikrobiellen Peptiden, PhagozytoseEntzug und der Abbau von Matrix-assoziierten Proteinen werden dem Immun-Inhibitor A zugeschrieben (Mukherjee et al., 2011). Der nur von P. larvae ERIC II kodierte ImmunInhibitor A wurde bislang keiner Analyse unterzogen, dabei stellt dieser einen potentiell entscheidenden Unterschied zwischen den Genotypen dar. Die Erstellung von ImmunInhibitor A-knock-out-Mutanten wäre dementsprechend für eine Analyse der ERIC IIspezifischen AFB-Pathogenese anzuraten. 


\section{Referenzen}

Adams, V., Li, J., Wisniewski, J. A., Uzal, F. A., Moore, R. J., et al. (2014). Virulence Plasmids of Spore-Forming Bacteria. Microbiol. Spectr. 2, PLAS-0024-2014.

Ahn, J.-H., Hong, I.-P., Bok, J.-I., Kim, B.-Y., Song, J., et al. (2012). Pyrosequencing analysis of the bacterial communities in the guts of honey bees Apis cerana and Apis mellifera in Korea. J. Microbiol. 50, 735-45.

Alippi, A. (1999). "Bacterial diseases," in Bee disease diagnosis, eds. M. Colin, B. Ball, und M. Kilani (Zaragoza: CIHEAM), 31-59.

Arai, R., Tominaga, K., Wu, M., Okura, M., Ito, K., et al. (2012). Diversity of Melissococcus plutonius from Honeybee Larvae in Japan and Experimental Reproduction of European Foulbrood with Cultured Atypical Isolates. PLoS One 7, e33708.

Ashida, H., Maki, R., Ozawa, H., Tani, Y., Kiyohara, M., et al. (2008). Characterization of two different endo-alpha- $N$-acetylgalactosaminidases from probiotic and pathogenic enterobacteria, Bifidobacterium longum and Clostridium perfringens. Glycobiology $18,727-34$.

Bailey, L. (1957a). European Foul Brood: a Disease of the Larval Honeybee (Apis mellifera L.) caused by a Combination of Streptococcus pluton (Bacillus pluton White) and Bacterium eurydice White. Nature 180, 1214-1215.

Bailey, L. (1957b). The Isolation and Cultural Characteristics of Streptococcus pluton and further Oberservations on Bacterium eurydice. J. gen. Microbiol. 17, 39-48.

Bailey, L. (1963a). The Habitat of Bacterium eurydice. J. gen. Microbiol. 31, 147-150.

Bailey, L. (1963b). The Pathogenicity for Honey-Bee Larvae of Microorganisms Associated with European Foulbrood. J. Insect Pathol. 5, 198-205.

Bailey, L. (1983). Melissococcus pluton, the cause of European foulbrood of honey bees (Apis spp.). J. Appl. Microbiol. 55, 65-69.

Bailey, L., Gibbs, A. J. (1962). Cultural Characters of Streptococcus pluton and its Differentiation from Associated Enterococci. J. gen. Microbiol. 28, 385-391.

Barsby, T., Kelly, M. T., Andersen, R. J. (2002). Tupuseleiamides and Basiliskamides, New Acyldipeptides and Antifungal Polyketides Produced in Culture by a Bacillus laterosporus Isolate Obtained from a Tropical Marine Habitat. J. Nat. Prod. 65, $1447-1451$.

Barsby, T., Kelly, M. T., Gagné, S. M., Andersen, R. J. (2001). Bogorol A Produced in Culture by a Marine Bacillus sp. Reveals a Novel Template for Cationic Peptide Antibiotics. Org. Lett. 3, 437-440. 
Barsby, T., Warabi, K., Sørensen, D., Zimmerman, W. T., Kelly, M. T., et al. (2006). The Bogorol family of antibiotics: template-based structure elucidation and a new approach to positioning enantiomeric pairs of amino acids. J. Org. Chem. 71, 6031-7.

Bennett, G. M., Mccutcheon, J. P., MacDonald, B. R., Romanovicz, D., Moran, N. A. (2014). Differential genome evolution between companion symbionts in an insectbacterial symbiosis. MBio 5, e01697-14.

Billington, S. J., Jost, B. H., Songer, J. G. (2000). Thiol-activated cytolysins: structure, function and role in pathogenesis. FEMS Microbiol. Lett. 182, 197-205.

Blackburn, M. B., Martin, P. A. W., Kuhar, D., Farrar, R. R., Gundersen-Rindal, D. E. (2011). The occurrence of photorhabdus-like toxin complexes in Bacillus thuringiensis. PLoS One 6, e18122.

Brüssow, H., Canchaya, C., Hardt, W., Bru, H. (2004). Phages and the Evolution of Bacterial Pathogens: from Genomic Rearrangements to Lysogenic. Microbiol. Mol. Biol. Rev. 68, 560-602.

Butler, E., Alsterfjord, M., Olofsson, T. C., Karlsson, C., Malmström, J., et al. (2013). Proteins of novel lactic acid bacteria from Apis mellifera mellifera: an insight into the production of known extra-cellular proteins during microbial stress. BMC Microbiol. $13,235$.

Cai, J., Collins, M. D. (1994). Evidence for a close phylogenetic relationship between Melissococcus pluton, the causative agent of European foulbrood disease, and the genus Enterococcus. Int. J. Syst. Bacteriol. 44, 365-7.

Cartron, M. L., Maddocks, S., Gillingham, P., Craven, C. J., Andrews, S. C. (2006). Feo Transport of ferrous iron into bacteria. BioMetals 19, 143-157.

Cheshire, F. R., Cheyne, W. W. (1885). The pathogenic history and history under cultivation of a new bacillus (B. alvei), the cause of a disease of the hive bee hitherto known as foulbrood. J. Roy. Microsc. Soc. 5, 581-601.

Chowdhury, S. A., Nallapareddy, S. R., Arias, C. A., Murray, B. E. (2014). The majority of a collection of U.S. endocarditis Enterococcus faecalis isolates obtained from 1974 to 2004 lack capsular genes and belong to diverse, non-hospital-associated lineages. $J$. Clin. Microbiol. 52, 549-556.

Chung, M.-C., Popova, T. G., Millis, B. A., Mukherjee, D. V, Zhou, W., et al. (2006). Secreted neutral metalloproteases of Bacillus anthracis as candidate pathogenic factors. J. Biol. Chem. 281, 31408-18.

Courtney, H. S., Li, Y., Dale, J. B., Hasty, D. L. (1994). Cloning, sequencing, and expression of a fibronectin/fibrinogen-binding protein from group A streptococci. Infect. Immun. 62, 3937-46.

Cross, A. S. (1990). The biologic significance of bacterial encapsulation. Curr. Top. Microbiol. Immunol. 150, 87-95. 
Desjardine, K., Pereira, A., Wright, H., Matainaho, T., Kelly, M., et al. (2007). Tauramamide, a lipopeptide antibiotic produced in culture by Brevibacillus laterosporus isolated from a marine habitat: structure elucidation and synthesis. $J$. Nat. Prod. 70, 1850-3.

Dzierzon, J. (1882). Rational bee-keeping. London: Houlston and Sons.

Edwards, C. G., Haag, K. M., Collins, M. D., Hutson, R. A., Huang, Y. C. (1998). Lactobacillus kunkeei sp. nov.: a spoilage organism associated with grape juice fermentations. J. Appl. Microbiol. 84, 698-702.

Endo, A., Irisawa, T., Futagawa-Endo, Y., Sonomoto, K., Itoh, K., et al. (2011). Fructobacillus tropaeoli sp. nov., a fructophilic lactic acid bacterium isolated from a flower. Int. J. Syst. Evol. Microbiol. 61, 898-902.

Endo, A., Salminen, S. (2013). Honeybees and beehives are rich sources for fructophilic lactic acid bacteria. Syst. Appl. Microbiol. 36, 444-448.

Engel, P., Martinson, V. G., Moran, N. A. (2012). Functional diversity within the simple gut microbiota of the honey bee. Proc. Natl. Acad. Sci. U. S. A. 109, 11002-7.

Fang, S., Wang, L., Guo, W., Zhang, X., Peng, D., et al. (2009). Bacillus thuringiensis bel protein enhances the toxicity of Cry1 Ac protein to Helicoverpa armigera larvae by degrading insect intestinal mucin. Appl. Environ. Microbiol. 75, 5237-43.

Forsgren, E. (2010). European foulbrood in honey bees. J. Invertebr. Pathol. 103 Suppl, S5-9.

Frost, L. S., Leplae, R., Summers, A. O., Toussaint, A. (2005). Mobile genetic elements: the agents of open source evolution. Nat. Rev. Microbiol. 3, 722-32.

Fünfhaus, A., Poppinga, L., Genersch, E. (2013). Identification and characterization of two novel toxins expressed by the lethal honey bee pathogen Paenibacillus larvae, the causative agent of American foulbrood. Environ. Microbiol. 15, 2951-2965.

Garcia-Gonzalez, E., Genersch, E. (2013). Honey bee larval peritrophic matrix degradation during infection with Paenibacillus larvae, the aetiological agent of American foulbrood of honey bees, is a key step in pathogenesis. Environ. Microbiol. 15, 2894 2901.

Garcia-Gonzalez, E., Müller, S., Ensle, P., Süssmuth, R. D., Genersch, E. (2014a). Elucidation of sevadicin, a novel non-ribosomal peptide secondary metabolite produced by the honey bee pathogenic bacterium Paenibacillus larvae. Environ. Microbiol. 16, 1297-309.

Garcia-Gonzalez, E., Müller, S., Hertlein, G., Heid, N., Süssmuth, R. D., et al. (2014b). Biological effects of paenilamicin, a secondary metabolite antibiotic produced by the honey bee pathogenic bacterium Paenibacillus larvae. Microbiologyopen 3, 642-656. 
Garcia-Gonzalez, E., Poppinga, L., Fünfhaus, A., Hertlein, G., Hedtke, K., et al. (2014c). Paenibacillus larvae chitin-degrading protein PICBP49 is a key virulence factor in American Foulbrood of honey bees. PLoS Pathog. 10, e1004284.

Genersch, E. (2008). Paenibacillus larvae and American Foulbrood - Long since known and still surprising. J. fur Verbraucherschutz und Leb. 3, 429-434.

Genersch, E. (2010a). American Foulbrood in honeybees and its causative agent, Paenibacillus larvae. J. Invertebr. Pathol. 103, S10-9.

Genersch, E. (2010b). Honey bee pathology: current threats to honey bees and beekeeping. Appl. Microbiol. Biotechnol. 87, 87-97.

Geoffroy, C., Mengaud, J., Alouf, J. E., Cossart, P. (1990). Alveolysin, the thiol-activated toxin of Bacillus alvei, is homologous to listeriolysin $\mathrm{O}$, perfringolysin $\mathrm{O}$, pneumolysin, and streptolysin $\mathrm{O}$ and contains a single cysteine. J. Bacteriol. 172, 7301-5.

Gerard, J., Haden, P., Kelly, M. T., Andersen, R. J. (1996). Loloatin B, A cyclic decapeptide antibiotic produced in culture by a tropical marine bacterium. Tetrahedron Lett. 37, 7201-7204.

Gerard, J. M., Haden, P., Kelly, M. T., Andersen, R. J. (1999). Loloatins A-D, cyclic decapeptide antibiotics produced in culture by a tropical marine bacterium. J. Nat. Prod. 62, 80-5.

Goodhead, I., Darby, A. C. (2015). Taking the pseudo out of pseudogenes. Curr. Opin. Microbiol. 23, 102-109.

Graveline, R., Segura, M., Radzioch, D., Gottschalk, M. (2007). TLR2-dependent recognition of Streptococcus suis is modulated by the presence of capsular polysaccharide which modifies macrophage responsiveness. Int. Immunol. 19, 375389.

Gregg, K. J., Boraston, A. B. (2009). Cloning, recombinant production, crystallization and preliminary X-ray diffraction analysis of a family 101 glycoside hydrolase from Streptococcus pneumoniae. Acta Crystallogr. Sect. F Struct. Biol. Cryst. Commun. 65, 133-135.

Guder, A., Wiedemann, I., Sahl, H. G. (2000). Posttranslationally modified bacteriocins-the lantibiotics. Biopolymers 55, 62-73.

Hancock, L. E., Gilmore, M. S. (2002). The capsular polysaccharide of Enterococcus faecalis and its relationship to other polysaccharides in the cell wall. Proc. Natl. Acad. Sci. U. S. A. 99, 1574-1579.

Hertlein, G., Müller, S., Garcia-Gonzalez, E., Poppinga, L., Süssmuth, R. D., et al. (2014). Production of the Catechol Type Siderophore Bacillibactin by the Honey Bee Pathogen Paenibacillus larvae. PLoS One 9, e108272. 
Heyndrickx, M., Vandemeulebroecke, K., Hoste, B., Janssen, P., Kersters, K., et al. (1996). Reclassification of Paenibacillus (formerly Bacillus) pulvifaciens (Nakamura 1984) Ash et al. 1994, a later subjective synonym of Paenibacillus (formerly Bacillus) larvae (White 1906) Ash et al. 1994, as a subspecies of P. larvae, with emended description. Int. J. Syst. Bacteriol. 46, 270-279.

Holmes, A. R., McNab, R., Millsap, K. W., Rohde, M., Hammerschmidt, S., et al. (2001). The pavA gene of Streptococcus pneumoniae encodes a fibronectin-binding protein that is essential for virulence. Mol. Microbiol. 41, 1395-408.

Hroncova, Z., Havlik, J., Killer, J., Doskocil, I., Tyl, J., et al. (2015). Variation in Honey Bee Gut Microbial Diversity Affected by Ontogenetic Stage, Age and Geographic Location. PLoS One 10, e0118707.

Jiménez, E., Ladero, V., Chico, I., Maldonado-Barragán, A., López, M., et al. (2013). Antibiotic resistance, virulence determinants and production of biogenic amines among enterococci from ovine, feline, canine, porcine and human milk. $B M C$ Microbiol. 13, 288.

Jung, C. M., Matsushita, O., Katayama, S., Minami, J., Sakurai, J., et al. (1999). Identification of metal ligands in the Clostridium histolyticum ColH collagenase. J. Bacteriol. 181, 2816-22.

Kanbar, G., Engels, W., Nicholson, G., Hertle, R., Winkelmann, G. (2005). Corrigendum to: Tyramine functions as a toxin in honey bee larvae during -transmitted infection by Melissococcus plutonius [FEMS Microbiol. Lett. 234 (2004) 149-154]. FEMS Microbiol. Lett. 245, 193-193.

Kanbar, G., Engels, W., Nicholson, G. J., Hertle, R., Winkelmann, G. (2004). Tyramine functions as a toxin in honey bee larvae during Varroa-transmitted infection by Melissococcus pluton. FEMS Microbiol. Lett. 234, 149-54.

Klaenhammer, T. R. (1988). Bacteriocins of lactic acid bacteria. Biochimie 70, 337-349.

Klein, A.-M., Vaissière, B. E., Cane, J. H., Steffan-Dewenter, I., Cunningham, S. A., et al. (2007). Importance of pollinators in changing landscapes for world crops. Proc. Biol. Sci. 274, 303-13.

Kooi, C., Subsin, B., Chen, R., Pohorelic, B., Sokol, P. A. (2006). Burkholderia cenocepacia $\mathrm{ZmpB}$ is a broad-specificity zinc metalloprotease involved in virulence. Infect. Immun. 74, 4083-4093.

Krewulak, K. D., Vogel, H. J. (2008). Structural biology of bacterial iron uptake. Biochim. Biophys. Acta - Biomembr. 1778, 1781-1804.

Lee, J. Y., Passalacqua, K. D., Hanna, P. C., Sherman, D. H. (2011). Regulation of petrobactin and bacillibactin biosynthesis in Bacillus anthracis under iron and oxygen variation. PLoS One 6, e20777. 
Li, S., Kelly, S. J., Lamani, E., Ferraroni, M., Jedrzejas, M. J. (2000). Structural basis of hyaluronan degradation by Streptococcus pneumoniae hyaluronate lyase. EMBO J. $19,1228-40$.

Lindsay, A.-M., Zhang, M., Mitchell, Z., Holden, M. T. G., Waller, A. S., et al. (2009). The Streptococcus equi prophage-encoded protein SEQ2045 is a hyaluronan-specific hyaluronate lyase that is produced during equine infection. Microbiology 155, 443-9.

Logan, N. A., Forsyth, G., Lebbe, L., Goris, J., Heyndrickx, M., et al. (2002). Polyphasic identification of Bacillus and Brevibacillus strains from clinical, dairy and industrial specimens and proposal of Brevibacillus invocatus sp. nov.. Int. J. Syst. Evol.

Microbiol. 52, 953-66.

Lövgren, A., Zhang, M., Engström, A., Dalhammar, G., Landén, R. (1990). Molecular characterization of Immune Inhibitor A, a secreted virulence protease from Bacillus thuringiensis. Mol. Microbiol. 4, 2137-2146.

Luo, X., Chen, L., Huang, Q., Zheng, J., Zhou, W., et al. (2013). Bacillus thuringiensis metalloproteinase Bmp1 functions as a nematicidal virulence factor. Appl. Environ. Microbiol. 79, 460-468.

Markowitz, V. M., Mavromatis, K., Ivanova, N. N., Chen, I.-M. A., Chu, K., et al. (2009). IMG ER: a system for microbial genome annotation expert review and curation. Bioinformatics 25, 2271-8.

Martinson, V. G., Danforth, B. N., Minckley, R. L., Rueppell, O., Tingek, S., et al. (2011). A simple and distinctive microbiota associated with honey bees and bumble bees. Mol. Ecol. 20, 619-28.

Massey, R. C., Kantzanou, M. N., Fowler, T., Day, N. P. J., Schofield, K., et al. (2001). Fibronectin-binding protein A of Staphylococcus aureus has multiple, substituting, binding regions that mediate adherence to fibronectin and invasion of endothelial cells. Cell. Microbiol. 3, 839-851.

McCutcheon, J. P., Moran, N. A. (2011). Extreme genome reduction in symbiotic bacteria. Nat. Rev. Microbiol. 10, 13-26.

Meusch, D., Gatsogiannis, C., Efremov, R. G., Lang, A. E., Hofnagel, O., et al. (2014). Mechanism of Tc toxin action revealed in molecular detail. Nature 508, 61-5.

Miethke, M., Klotz, O., Linne, U., May, J. J., Beckering, C. L., et al. (2006). Ferribacillibactin uptake and hydrolysis in Bacillus subtilis. Mol. Microbiol. 61, 14131427.

Moran, N. A. (2002). Microbial minimalism: Genome reduction in bacterial pathogens. Cell 108, 583-586.

Moran, N. A. (2015). Genomics of the honey bee microbiome. Curr. Opin. Insect Sci. 10, $22-28$. 
Mukherjee, D. V., Tonry, J. H., Kim, K. S., Ramarao, N., Popova, T. G., et al. (2011). Bacillus anthracis protease InhA increases blood-brain barrier permeability and contributes to cerebral hemorrhages. PLoS One 6, e17921.

Müller, S., Garcia-Gonzalez, E., Genersch, E., Süssmuth, R. D. (2015). Involvement of secondary metabolites in the pathogenesis of the American foulbrood of honey bees caused by Paenibacillus larvae. Nat. Prod. Rep. 32, 765-78.

Neveling, D. P., Endo, A., Dicks, L. M. T. (2012). Fructophilic Lactobacillus kunkeei and Lactobacillus brevis isolated from fresh flowers, bees and bee-hives. Curr. Microbiol. $65,507-15$.

Nicholson, W. L., Fajardo-Cavazos, P., Rebeil, R., Slieman, T. A., Riesenman, P. J., et al. (2002). Bacterial endospores and their significance in stress resistance. Antonie van Leeuwenhoek, Int. J. Gen. Mol. Microbiol. 81, 27-32.

Nilsson, A. I., Koskiniemi, S., Eriksson, S., Kugelberg, E., Hinton, J. C. D., et al. (2005). Bacterial genome size reduction by experimental evolution. Proc. Natl. Acad. Sci. U. S. A. 102, 12112-12116.

Okumura, K., Arai, R., Okura, M., Kirikae, T., Takamatsu, D., et al. (2011). Complete genome sequence of Melissococcus plutonius ATCC 35311. J. Bacteriol. 193, 402930 .

Olaitan, P. B., Adeleke, O. E., Ola, I. O. (2007). Honey: A reservoir for microorganisms and an inhibitory agent for microbes. Afr. Health Sci. 7, 159-165.

Oliveira, A., Melo, L. D. R., Kropinski, A. M., Azeredo, J. (2013). Complete Genome Sequence of the Broad-Host-Range Paenibacillus larvae Phage phiIBB_Pl23. Genome Announc. 1, e00438-13-e00438-13.

Olofsson, T. C., Vásquez, A. (2008). Detection and identification of a novel lactic acid bacterial flora within the honey stomach of the honeybee Apis mellifera. Curr. Microbiol. 57, 356-63.

De Palencia, P. F., Fernández, M., Mohedano, M. L., Ladero, V., Quevedo, C., et al. (2011). Role of tyramine synthesis by food-borne Enterococcus durans in adaptation to the gastrointestinal tract environment. Appl. Environ. Microbiol. 77, 699-702.

Palmer, K. L., Godfrey, P., Griggs, A., Kos, V. N., Zucker, J., et al. (2012). Comparative Genomics of Enterococci: Variation in Enterococcus faecalis, Clade Structure in E. faecium, and Defining Characteristics of E. gallinarum and E. casseliflavus. MBio 3, e00318-11-e00318-11.

Perez, R. H., Zendo, T., Sonomoto, K. (2014). Novel bacteriocins from lactic acid bacteria (LAB): various structures and applications. Microb. Cell Fact. 13 Suppl 1, S3.

Perin, L. M., Miranda, R. O., Todorov, S. D., Franco, B. D. G. de M., Nero, L. A. (2014). Virulence, antibiotic resistance and biogenic amines of bacteriocinogenic lactococci and enterococci isolated from goat milk. Int. J. Food Microbiol. 185, 121-126. 
Pessanha, R. R., Carramaschi, I. N., dos Santos Mallet, J. R., Queiroz, M. M. C., Zahner, V. (2015). Evaluation of larvicidal activity and effects on post embrionary development of laboratory reared Lucilia cuprina (Wiedemann, 1830) (Diptera: Calliphoridae), treated with Brevibacillus laterosporus. J. Invertebr. Pathol. 128, 4446.

Peterson, P. K., Wilkinson, B. J., Kim, Y., Schmeling, D., Quie, P. G. (1978). Influence of encapsulation on staphylococcal opsonization and phagocytosis by human polymorphonuclear leukocytes. Infect. Immun. 19, 943-949.

Petit, L., Maier, E., Gibert, M., Popoff, M. R., Benz, R. (2001). Clostridium perfringens epsilon toxin induces a rapid change of cell membrane permeability to ions and forms channels in artificial lipid bilayers. J. Biol. Chem. 276, 15736-40.

Poppinga, L., Genersch, E. (2015). Molecular pathogenesis of American Foulbrood: how Paenibacillus larvae kills honey bee larvae. Curr. Opin. Insect Sci., 1-8.

Poppinga, L., Janesch, B., Fünfhaus, A., Sekot, G., Garcia-Gonzalez, E., et al. (2012). Identification and functional analysis of the S-layer protein SplA of Paenibacillus larvae, the causative agent of American Foulbrood of honey bees. PLoS Pathog. 8, e1002716.

Prasanna, L., Eijsink, V. G. H., Meadow, R., Gåseidnes, S. (2013). A novel strain of Brevibacillus laterosporus produces chitinases that contribute to its biocontrol potential. Appl. Microbiol. Biotechnol. 97, 1601-11.

Rigottier-Gois, L., Madec, C., Navickas, A., Matos, R. C., Akary-Lepage, E., et al. (2014). The Surface Rhamnopolysaccharide Epa of Enterococcus faecalis Is a Key Determinant of Intestinal Colonization. J. Infect. Dis. 211, 62-71.

Ritter, W. (2012). Bienen gesund erhalten. Stuttgart: Eugen Ulmer KG.

Rocha, E. P. C., Danchin, A. (2002). Base composition bias might result from competition for metabolic resources. Trends Genet. 18, 291-294.

Rokop, Z. P., Horton, M. A., Newton, I. L. G. (2015). Interactions between co-occurring lactic acid bacteria in the honey bee hive. Appl. Environ. Microbiol. In Druck.

Ruiu, L. (2013). Brevibacillus laterosporus, a Pathogen of Invertebrates and a BroadSpectrum Antimicrobial Species. Insects 4, 476-492.

Ruiu, L., Satta, A., Floris, I. (2012). Observations on house fly larvae midgut ultrastructure after Brevibacillus laterosporus ingestion. J. Invertebr. Pathol. 111, 211-6.

Schimke, R. T., Berlin, C. M., Sweeney, E. W., Carroll, W. R. (1966). The generation of energy by the arginine dihydrolase pathway in Mycoplasma hominis 07. J. Biol. Chem. 241, 2228-2236.

Shimanuki, H. (1990). "Bacteria," in Honey Bee Pests, Predators, and Diseases, eds. R. Morse und R. Nowogrodzki (New York: Cornell University Press), 27-47. 
Shrivastava, K. P. (1982). Bacterium (Lactobacillus?) eurydice strains from bumble bees. J. Invertebr. Pathol. 40, 180-185.

Simon, N. C., Aktories, K., Barbieri, J. T. (2014). Novel bacterial ADP-ribosylating toxins: structure and function. Nat. Rev. Microbiol. 12, 599-611.

Spigaglia, P., Barketi-Klai, A., Collignon, A., Mastrantonio, P., Barbanti, F., et al. (2013). Surface-layer (S-layer) of human and animal Clostridium difficile strains and their behaviour in adherence to epithelial cells and intestinal colonization. J. Med. Microbiol. 62, 1386-93.

Steffan-Dewenter, I., Potts, S. G., Packer, L., Ghazoul, J. (2005). Pollinator diversity and crop pollination services are at risk [3] (multiple letters). Trends Ecol. Evol. 20, 651653.

Tellam, R. L., Wijffels, G., Willadsen, P. (1999). Peritrophic matrix proteins. Insect Biochem. Mol. Biol. 29, 87-101.

Teng, F., Singh, K. V., Bourgogne, A., Zeng, J., Murray, B. E. (2009). Further characterization of the epa gene cluster and Epa polysaccharides of Enterococcus faecalis. Infect. Immun. 77, 3759-3767.

Terra, W. R. (2001). The origin and functions of the insect peritrophic membrane and peritrophic gel. Arch. Insect Biochem. Physiol. 47, 47-61.

Thurlow, L. R., Thomas, V. C., Hancock, L. E. (2009). Capsular polysaccharide production in Enterococcus faecalis and contribution of $\mathrm{CpsF}$ to capsule serospecificity. J. Bacteriol. 191, 6203-6210.

Tian, B., Yang, J., Lian, L., Wang, C., Li, N., et al. (2007). Role of an extracellular neutral protease in infection against nematodes by Brevibacillus laterosporus strain G4. Appl. Microbiol. Biotechnol. 74, 372-80.

Toprak, U., Harris, S., Baldwin, D., Theilmann, D., Gillott, C., et al. (2012). Role of enhancin in Mamestra configurata nucleopolyhedrovirus virulence: selective degradation of host peritrophic matrix proteins. J. Gen. Virol. 93, 744-53.

Vásquez, A., Forsgren, E., Fries, I., Paxton, R. J., Flaberg, E., et al. (2012). Symbionts as major modulators of insect health: lactic acid bacteria and honeybees. PLoS One 7, e33188.

Vojvodic, S., Rehan, S. M., Anderson, K. E. (2013). Microbial gut diversity of Africanized and European honey bee larval instars. PLoS One 8, e72106.

Weber, T., Blin, K., Duddela, S., Krug, D., Kim, H. U., et al. (2015). antiSMASH 3.0--a comprehensive resource for the genome mining of biosynthetic gene clusters. Nucleic Acids Res. 43, 237-243.

Wessels, M. R., Moses, A. E., Goldberg, J. B., DiCesare, T. J. (1991). Hyaluronic acid capsule is a virulence factor for mucoid group A streptococci. Proc. Natl. Acad. Sci. U. S. A. 88, 8317-8321. 
White, G. (1906). The bacteria of the apiary, with special reference to bee diseases. USDA, Bur. Entomol. Tech. Ser., 1-50.

Wolf, Y. I., Koonin, E. V. (2013). Genome reduction as the dominant mode of evolution. BioEssays 35, 829-837.

Woodring, J., Boulden, M., Das, S., Gäde, G. (1993). Studies on blood sugar homeostasis in the honeybee (Apis mellifera, L.). J. Insect Physiol. 39, 89-97.

Yang, S.-C., Lin, C.-H., Sung, C. T., Fang, J.-Y. (2014). Antibacterial activities of bacteriocins: application in foods and pharmaceuticals. Front. Microbiol. 5, 241.

Yue, D., Nordhoff, M., Wieler, L. H., Genersch, E. (2008). Fluorescence in situ hybridization (FISH) analysis of the interactions between honeybee larvae and Paenibacillus larvae, the causative agent of American foulbrood of honeybees (Apis mellifera). Environ. Microbiol. 10, 1612-20.

Zhao, J., Guo, L., Zeng, H., Yang, X., Yuan, J., et al. (2012). Purification and characterization of a novel antimicrobial peptide from Brevibacillus laterosporus strain A60. Peptides 33, 206-211. 


\section{Zusammenfassung}

Die bakteriellen Erreger Paenibacillus larvae und Melissococcus plutonius verursachen mit der Amerikanischen Faulbrut (AFB) bzw. Europäischen Faulbrut (EFB) weltweit ernstzunehmende Bienenkrankheiten.

Aus einem Schweizer EFB-Ausbruch wurden drei typische M. plutonius Stämme isoliert. Mit Hilfe von Genomanalysen von einem atypischen und 13 typischen M. plutonius Stämmen konnten erstmals potentielle Virulenzfaktoren und Fitnessfaktoren für $M$. plutonius ermittelt werden, die dem Pathogen ermöglichen, eine Infektion hervorzurufen und die Larve abzutöten. Dazu zählen Bacteriocine, Tyrosin-Decarboxylase, Enhancin, ein PlCBP49-homologes Protein, Oberflächenproteine, Melissotoxin A, Collagenase, ein EpaHomolog, Kapsel, Proteasen, Glycosidhydrolasen und Polysaccharidlyasen. Zwei potentielle Virulenzfaktoren (Melissotoxin A und ein Matrixbindeprotein) der EFBPathogenese liegen plasmidkodierend vor. Bisherige Untersuchungen haben gezeigt, dass typische Stämme im Gegensatz zu atypischen Stämmen nach mehreren Anzuchten ihre Virulenz verlieren und den Tod der Larve nicht mehr hervorrufen können. Der Verlust der Virulenz bei typischen Stämmen könnte hauptsächlich auf den Verlust des Plasmids zurückzuführen sein. Des Weiteren konnte über die vergleichende Genomanalyse gezeigt werden, dass atypische und typische Stämme unterschiedliche Virulenzmechanismen entwickelt haben müssen. Der atypische Stamm M. plutonius DAT561 gilt als hochvirulent, obwohl dieser das Virulenzplasmid nicht besitzt. Das Fehlen dieses Plasmids kompensieren atypische Stämme wahrscheinlich über einen schnelleren Ressourcenverbrauch, welche dann der Larve dann zum Wachsen fehlen, und über zusätzliche Oberflächenproteine. Auch innerhalb der typischen Stämme konnten durch die vergleichende Genomanalyse unterschiedliche Virulenzgrade vorhergesagt werden, was hauptsächlich dem Fehlen von Endo-alpha- $N$-acetylgalactosaminidase, Tyrosin-Decarboxylase und/oder des Virulenzplasmids geschuldet sein sollte. Weiterhin wurde eine Expressionsanalyse mit drei Virulenzfaktoren durchgeführt (Endo-alpha- $N$-acetylgalactosaminidase, Enhancin und Melissotoxin A). Es konnte gezeigt werden, dass die Gene für die Virulenzfaktoren im Rahmen der Infektion in vivo exprimiert werden. Es wurde anhand der identifizierten Virulenzfaktoren ein Infektions- und Pathogenese-Modell erstellt.

Für P. larvae konnten neue Virulenzfaktoren über die vergleichende Genomanalyse von zwei verschiedenen Stämmen/Genotypen ermittelt werden, die zur AFB-Pathogenese beitragen. Dazu gehören Bacteriocine, NRPS- und NRPS/PKS-Biosynthese-Gencluster, 
Enhancin, Hyaluronidase, Immun Inhibitor A, Bacillolysin, Toxine, Oberflächenproteine und Proteasen. Unterschiede in der Virulenz beider Genotypen ließen sich auf eine unterschiedliche Ausstattung in Bezug auf die genannten Virulenzfaktoren zurückführen. Über diese Analysen konnte ein Infektionsmodell für die AFB erstellt werden.

Die Ergebnisse aus den Genomstudien von AFB-, EFB-Primär- und Sekundärerregern wurden genutzt, um die AFB- mit der EFB-Pathogenese zu vergleichen. Man kann beide in eine nicht-invasive Phase und eine invasive Phase unterteilen. In beiden Phasen konnten Unterschiede zwischen AFB und EFB gezeigt werden. Es wird postuliert, dass sich während der nicht-invasiven Phase der EFB die Primär- und Sekundärerreger massiv im Darm vermehren. P. larvae dagegen verdrängt in dieser Phase bereits Nahrungskonkurrenten. Die invasive Phase startet mit der Degradation der peritrophischen Matrix, zu der P. larvae und wahrscheinlich auch M. plutonius in der Lage sind. Dieser Schritt wird in der EFBPathogenese unter Umständen von Sekundärerregern durch die Produktion von Exoenzymen wie z.B. Chitinasen unterstützt. P. larvae zerstört in einem nächsten Schritt Epithelzellen und dringt über eine parazelluläre Route in das Haemocoel vor. Auch bei M. plutonius sind diese Schritte denkbar. Die Mitwirkung von Sekundärerregern (EFB) könnte in diesem Stadium zu einer erhöhten Virulenz und Larvenmortalität führen. In einem letzten Schritt degradiert $P$. larvae die Larvenüberreste. Dieser Schritt wird vermutlich während der EFB von den Sekundärerregern übernommen.

Ergänzend konnte mit der Genomanalyse von Lactobacillus kunkeei gezeigt werden, dass dieser über die Bildung von einzigartigen Oberflächenproteinen wahrscheinlich zur Biofilmbildung und/oder Bindung an den Honigmagen der Biene fähig ist. Zudem zeigte die Genomanalyse, dass dieses Bakterium über die Bildung von Bacteriocinen potentiell sein Habitat gegen Konkurrenten verteidigen kann. Da L. kunkeei als Antagonist zu M. plutonius angesehen wird, sollte die Wirkung dieser Bacteriocine auf M. plutonius in weiterführenden Analysen untersucht werden. 


\section{E Appendix}

Auf dem digitalen Datenträger (CD) befinden sich drei Ordner. Diese „Ergänzenden Daten“ gehören zu den Kapiteln B3, B4 und B7. 



\section{Danksagung}

Zu allererst möchte ich Herrn Prof. Dr. Daniel danken. Normalerweise wird einem Doktoranden ein Themengebiet "zugewiesen“ - in meinem Fall konnte ich Herrn Prof. Dr. Daniel mit einem von mir ausgewählten Themengebiet überzeugen und durfte mich sozusagen „austoben“. Vielen Dank für das Vertrauen, die Finanzierung und die Ratschläge während der Doktorarbeit.

Herrn Dr. Hoppert möchte ich für die Betreuung und auch für die Vorschläge während der Fortschrittsberichte danken.

Ein besonders großes Dankeschön geht an alle Mitglieder des „G2L“ und der „EG-Abteilung“, die mir mit Rat und Tat zur Seite gestanden haben. Dabei zählt nicht nur die Arbeit, sondern auch das entgegengebrachte Vertrauen in privaten Gesprächen. Besonders Katrin, Sandra, Robert, Sascha, Dominik, Steffi, Kathleen, Frauke, Florian, Jacqueline und Sonja will ich in diesem Sinne hervorheben. Vielen Dank für alles! (gerade Robert mit seinen immer aufmunternden Worten)

Einen äußerst großen, positiven Einfluss auf meine Arbeit hatten insbesondere die Personen John, Heiko, Andreas und Anja. John hat die Stimmung im Doktoranden-Office mit seinem speziellen Humor regelmäßig aufgelockert. Auch seine extrem hilfsbereite Natur war wahrlich nicht nur ein Segen für mich, sondern auch für so einige andere im Labor. Heiko kenne ich seit Beginn meines Studiums in Göttingen und uns begleitet eine enge Freundschaft. Zufälligerweise arbeiten wir auch schon recht lange in derselben Abteilung (auch wenn er mit seiner Familie gerade in Schottland ist). Ich habe schon immer seine Meinung geschätzt und freue mich sehr auf ein Wiedersehen! Andreas und Anja haben mir während der Promotion nicht nur auf freundschaftliche Art und Weise zur Seite gestanden, sie haben mich auch in meinem Tun und Schaffen unterstützt. Zudem hatten sie immer ein offenes Ohr für mich. Das tat einfach gut. Und es verbindet.

Vielen Dank euch allen!

Den unzähligen Imkern möchte ich für die Zusammenarbeit und Unterstützung danken. Genauso wie meinen ehemaligen Bachelorstudenten, die sehr fleißig waren und wirklich gute Arbeit geleistet haben. Vielen Dank!

Meinen Freunden danke ich in dem Sinne, dass sie mich auch auf andere Gedanken gebracht haben. Während (und vor allem am Ende) einer Doktorarbeit neigt man dazu, sich mit seinen Gedanken nur noch in einer Parallelwelt zu befinden. Da tut es gut, wenn man ab und zu wieder ins „echte Leben“ zurückgeholt wurde. Und einem auch gesagt wird, dass Wissenschaft nicht alles im Leben ist, was zählt.

Kommen wir nun zu dem letzten und wichtigsten Punkt: der Familie. Ich kann meinen Eltern für ihre Unterstützung nicht genug danken. Nicht nur, dass sie mir das Studium erst ermöglicht haben, sie hatten auch immer ein offenes Ohr für mich. Und wenn ich eines gelernt habe, dann: Ratschläge der Eltern sollte man befolgen (oder zumindest stark gewichten...). Sie haben anscheinend immer Recht, auch wenn man es nicht sofort sieht. Meinen Geschwistern Diana und Thomas möchte ich ebenso danken wie meinen Omas, die mich auch immer unterstützt haben. Das größte Dankeschön geht an meine Frau, Sandra, mit der ich nun eine eigene Familie gründe. Du bist für mich sozusagen „bester Kumpel“ und „die Liebe des Lebens" zugleich. Deine unzähligen, aufbauenden Worte haben mir sehr geholfen. Der Rückhalt war unbeschreiblich. Ich habe und werde immer die Zeit mit dir (und bald unserem Kind) genießen. Danke! 


\section{Lebenslauf}

\section{Persönliche Daten}

Name

Geburtsdatum

Geburtsort

Familienstand

Staatsangehörigkeit

Bildungsweg

Seit $11 / 2010$

$2009-2015$

$2008-2009$

2008

2004

2002

$1994-2002$

1990 - 1994
Marvin Djukic

18.05.1984

Leinefelde

ledig

Deutsch
Promotionsstudent an der Georg-August-Universität zu Göttingen, Institut für Mikrobiologie und Genetik, Abteilung für Genomanalyse

Anfertigen der Promotionsarbeit mit dem Titel:

"Funktionelle Genomanalyse bakterieller Erreger, assoziiert mit der Europäischen Faulbrut von Honigbienen."

Beschäftigt als wissenschaftliche Hilfskraft, an der Georg-August-Universität zu Göttingen, Institut für Mikrobiologie und Genetik, Abteilung für Genomanalyse

Erreichen des Hochschulgrades Diplom-Biologe

Diplomarbeit an der Georg-August-Universität zu Göttingen, Institut für Mikrobiologie und Genetik, Abteilung für Genomanalyse

Diplomarbeit trägt den Titel:

"Charakterisierung von Bakterien aus dem Darm der Honigbiene."

Bestehen der Hauptdiplomprüfungen (Georg-August-Universität zu Göttingen)

Bestehen der Vordiplomprüfungen (Georg-August-Universität zu Göttingen)

Zulassung zum Studium der Biologie an der Georg-August-

Universität zu Göttingen

Gottfried-Wilhelm-Leibniz-Gymnasium Leinefelde (Abschluss: Abitur)

Grundschule Leinefelde 


\title{
Promovierenden-Erklärung der Georg-August-Universität Göttingen
}

\author{
Name: $\quad$ Djukic, Marvin \\ Anschrift: $\quad$ Heiligenstädter Straße 46, OT Leinefelde, 37327 Leinefelde-Worbis
}

Ich beabsichtige, eine Dissertation zum Thema „Funktionelle Genomanalyse bakterieller Erreger, assoziiert mit der Europäischen Faulbrut von Honigbienen.“ an der Georg-AugustUniversität Göttingen anzufertigen. Dabei werde ich von Herrn Prof. Rolf Daniel betreut.

Ich gebe folgende Erklärung ab:

1. Die Gelegenheit zum vorliegenden Promotionsvorhaben ist mir nicht kommerziell vermittelt worden. Insbesondere habe ich keine Organisation eingeschaltet, die gegen Entgelt Betreuerinnen und Betreuer für die Anfertigung von Dissertationen sucht oder die mir obliegenden Pflichten hinsichtlich der Prüfungsleistungen für mich ganz oder teilweise erledigt.

2. Hilfe Dritter wurde bis jetzt und wird auch künftig nur in wissenschaftlich vertretbarem und prüfungsrechtlich zulässigem Ausmaß in Anspruch genommen. Insbesondere werden alle Teile der Dissertation selbst angefertigt; unzulässige fremde Hilfe habe ich dazu weder unentgeltlich noch entgeltlich entgegengenommen und werde dies auch zukünftig so halten.

3. Die Richtlinien zur Sicherung der guten wissenschaftlichen Praxis an der Universität Göttingen werden von mir beachtet.

4. Eine entsprechende Promotion wurde an keiner anderen Hochschule im In- oder Ausland beantragt; die eingereichte Dissertation oder Teile von ihr wurden nicht für ein anderes Promotionsvorhaben verwendet.

Mir ist bekannt, dass unrichtige Angaben die Zulassung zur Promotion ausschließen bzw. später zum Verfahrensabbruch oder zur Rücknahme des erlangten Grades führen.

Leinefelde, den 27.08.2015 